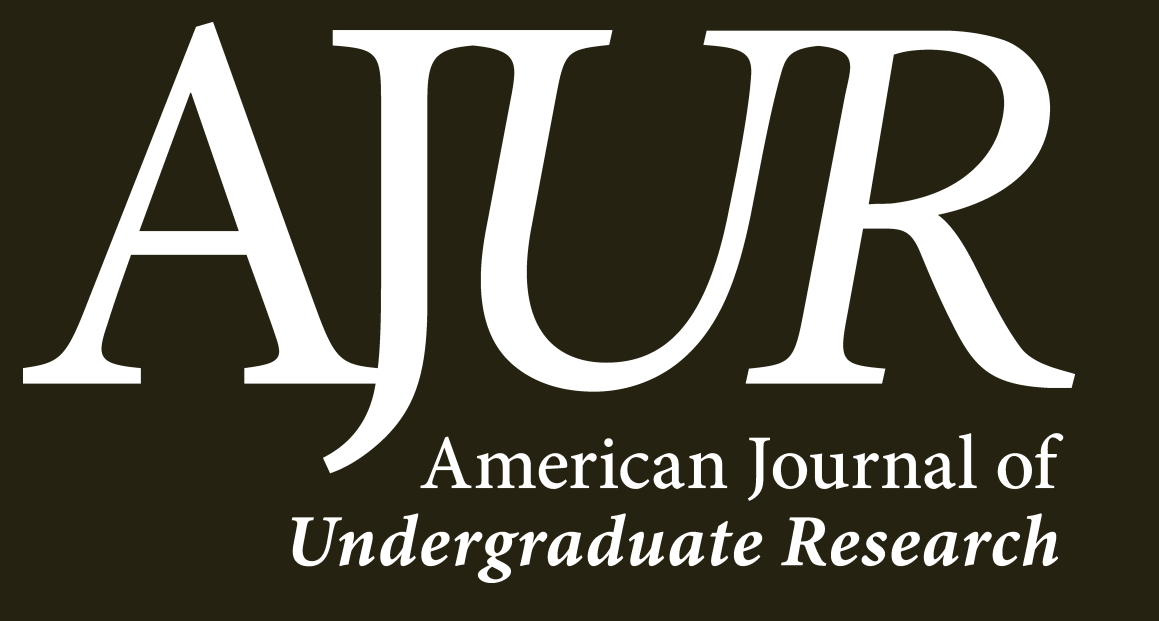

Volume 14 | Issue 3 | November 2017 www.ajuronline.org 


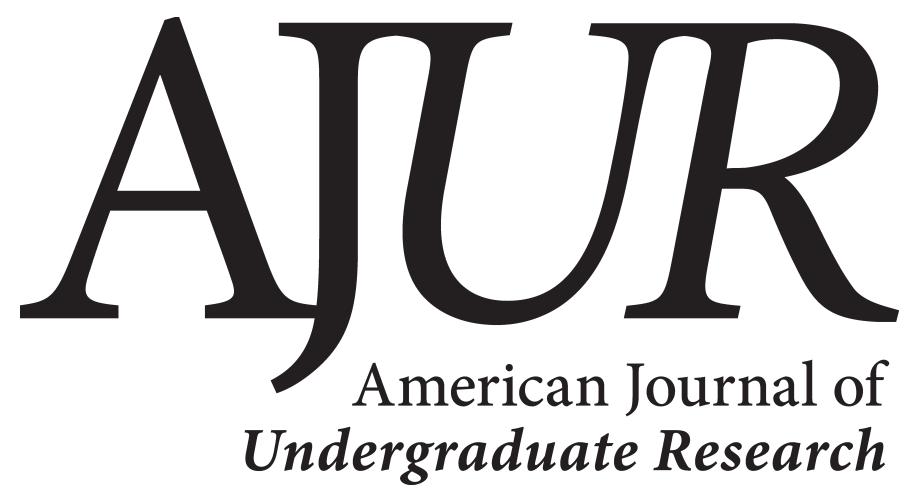

Volume 14 | Issue 3 | November 2017

www.ajuronline.org

2

3

5

45

69

85

101
AJUR History and Editorial Board

Special Thanks to AJUR's Sponsors

Strategies for Making Best Offers on eBay

William J. Britt, William E. Gryc, Jamie A. Oliva, Brittney N. Tuff, \& Charli E. White

The Relationship Between Parenting Styles and Substance Use Among University Students

Zackaria I. Niazi, Danielle Dick, Amy Adkins, \& Megan Cooke

Matricial Representations of Certain Finitely Presented Groups Generated by Order-2 Generators and Their Applications Ryan Golden \& Ilwoo Cho

Evolution of Leucyl-tRNA Synthetase Through Eukaryotic Speciation

Katelyn E. Unvert, Frank. A. Kovacs, Chi Zhang, Rachel A. Hellmann-Whitaker, \& Katelin N. Arndt

The Effect of $\mathrm{CO}_{2}$, Intracellular $\mathrm{pH}$, and Extracellular $\mathrm{pH}$ on Mechanosensory Proprioceptor Responses in Crayfish and Crab Viresh Dayaram, Cole Malloy, Sarah Martha, Brenda Alvarez, Ikenna Chukwudolue, Nadera Dabbain, Dlovan Mahmood, Slavina Goleva, Tori Hickey, Angel Ho, Molly King, Paige Kington, Matthew Mattingly, Samuel Potter, Landon Simpson, Amanda Spence, Henry Uradu, Jacob Van Doorn, dlovan faiq, \& Robin L. Cooper

Derivation of Explicit Solutions Describing Early Stages of Platelet Activation

Rachel Austin, Scott Fones, Dominic Santoleri, Kaitlyn Thomesen, \& Pak-Wing Fok 
American Journal of Undergraduate Research (AJUR) is a national, peer-reviewed, open-source, quarterly, multidisciplinary student research journal. It is indexed internationally by EBSCO, and is listed via the Library of Congress under ISSNs of 1536-4585 (for print) and 2375-8732 (for web). The journal was established in 2002.

EDITORIAL TEAM Volume 14 / Issue 3 / November 2017

Dr. Kestutis G. Bendinskas, Editor, editor@ajuronline.org

Dr. Anthony Contento, Technical Editor

Rose Throop, Print

Daniel Laird, Web Master

Dr. Bonita Graham, LaTex and Copy Editor

EDITORIAL BOARD by subject area

ACCOUNTING

Dr. Dean Crawford, dean.crawford@oswego.edu

\section{ART HISTORY}

Dr. Lisa Seppi,

lisa.seppi@oswego.edu

ASTROPHYSICS

Dr. Shashi Kanbur,

shashi.kanbur@oswego.edu

BEHAVIORAL NEUROSCIENCE

Dr. Aileen M. Bailey,

ambailey@smom.edu

\section{BIOCHEMISTRY}

Dr. Pamela K. Kerrigan, pamela.kerrigan@mountsaintvincent.edu

Dr. Nin Dingra,

ndingra@alaska.edu

BIOENGINEERING

Dr. Jorge I. Rodriguez, jorger@clemson.edu

BIOINFORMATICS

Dr. Kevin Daimi,

daimikj@udmercy.edu

Dr. John R. Jungck,

jungck@udel.edu

Dr. Isabelle Bichindaritz,

ibichind@oswego.edu

BIOLOGY, PHYSIOLOGY

Dr. David Dunn,

david.dunn@oswego.edu

BIOLOGY, DEVELOPMENTAL

Dr. Poongodi Geetha-Loganathan,

p.geethaloganathan@oswego.edu

\section{BIOLOGY, MICROBIOLOGY}

$$
\text { Dr. Peter Newell, }
$$

peternewell@oswego.edu

\section{BOTANY}

Dr. William R. Bromer, wbromer@stfrancis.edu

Dr. Julien Bachelier, julien.bacbelier@fu-berlin.de

\begin{abstract}
CHEMISTRY
Dr. Alfredo Castro,

castroa@felician.edu

Dr. Charles Kriley,

cekriley@gcc.edu

Dr. Douglas Mulford,

douglas.mulford@emory.edu

Dr. Vadoud Niri,

vadoud.niri@oswego.edu
\end{abstract}

COMMUNICATION DISORDERS AND SCIENCES

Dr. Kim Tillery,

Kim.Tillery@fredonia.edu

COMMUNICATION STUDIES

Dr. Jennifer Gerometta,

jgerometta@iona.edu

COMPUTER SCIENCES

Dr. Dele Oluwade,

deleoluwade@yahoo.com

Dr. Kevin Daimi,

daimikj@udmercy.edu

Dr. Levent Ertaul,

levent.ertaul@csueastbay.edu

Dr. Mais W Nijim,

Mais.Nijim@tamuk.edu

COMPUTATIONAL CHEMISTRY

Dr. Alexander Soudackor

asouda@illinois.edu

ECOLOGY

Dr. William R. Bromer,

wbromer@stfrancis.edu

ECONOMICS

Dr. Elizabeth Schmitt,

elizabeth.schmitt@oswego.edu

EDUCATION

Dr. Marcia Burrell,

marcia.burrell@oswego.edu

EDUCATION, PHYSICS

Dr. Andrew D. Gavrin,

agavrin@iupui.edu

ENGINEERING, ELECTRICAL

Dr. Michael Omidiora,

michael.omidiora@nyu.edu
ENGINEERING, MANUFACTURING AND CONSTRUCTION, ROBOTICS

Dr. Haoyu Wang,

wanghao@mail.ccsu.edu

ENGINEERING, SOFTWARE

Dr. Kevin Daimi,

daimikj@udmercy.edu

ENVIRONMENTAL SCIENCES

Dr. Eileen M. Cashman,

eileen.cashman@bumboldt.edu

FILM AND MEDIA STUDIES

Dr. Lauren Steimer,

lsteimer@mailbox.sc.edu

\section{HISTORY}

Dr. Richard Weyhing,

richard.weybing@oswego.edu

Dr. Murat Yasar,

muratyasar@oswego.edu

HONORARY EDITORIAL BOARD MEMBER

Dr. Lorrie Clemo,

lorrie.a.clemo@gmail.com

KINESIOLOGY / EXERCISE SCI-

$$
\text { ENCE }
$$

Dr. David Senchina,

david.senchina@drake.edu

LITERARY STUDIES

Dr. Douglas Guerra,

douglas.guerra@oswego.edu

\section{MATHEMATICS}

Dr. John Emert,

emert@bsu.edu

Dr. Jeffrey J. Boats,

boatsjj@udmercy.edu

Dr. J.D. Phillips,

jophilli@nmu.edu

Dr. Dele Oluwade,

deleoluwade@yahoo.com

Dr. Christopher Baltus,

christopher.baltus@oswego.edu

Dr. Mark Baker,

mark.baker@oswego.edu
MEDICAL SCIENCES

Joan Newell, MD

joannewellmd@gmail.com

METEOROLOGY

Dr. Steven Skubis,

steven.skubis@oswego.edu

MUSIC

Dr. Juliet Forshaw,

juliet.forshaw@oswego.edu

NANOSCIENCE AND CHEMISTRY

Dr. Gary Baker,

bakergar@missouri.edu

NEUROSCIENCE

Dr. Pamela E. Scott-Johnson, pscottj@calstatela.edu

Dr. Amy Overman,

aoverman@elon.edu

\section{PHYSICS}

Dr. Carolina Ilie, carolina.ilie@oswego.edu Dr. Mohammad Islam,

mohammad.islam@oswego.edu

POLITICAL SCIENCE

Dr. Katia Levintova,

levintoe@uwgb.edu

PSYCHOLOGY

Dr. Amy Overman, aoverman@elon.edu

Dr. Pamela E. Scott-Johnson, pscottj@calstatela.edu

STATISTICS

Dr. Mark Ecker, mark.ecker@uni.edu

TECHNOLOGY, ENGINEERING

Dr. Recayi Pecen, regpecen@na.edu 


\section{SPECIAL THANKS}

AJUR is made possible through the assistance of our sponsors.

Support for this issue has been provided by Wiley

as well as the Office of the Provost at

the State University of New York at Oswego. Thank you!

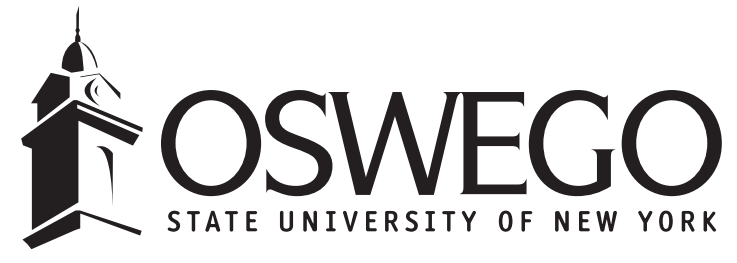

Interested in supporting quality undergraduate research?

Request sponsorship information at editor@ajuronline.org 


\title{
Strategies for Making Best Offers on eBay ${ }^{a}$
}

William J. Britt, William E. Gryc*, Jamie A. Oliva, Brittney N. Tuff, Charli E. White

Department of Mathematics and Computer Science, Mublenberg College, Allentown, PA

Students: wb248640@mublenberg.edu, jo248428@mublenberg.edu, bt247582@mublenberg.edu, cw248964@mublenberg.edu Mentor:wgryc@mublenberg.edu*

\begin{abstract}
We model for "Buy-It-Now or Best Offer" auctions on eBay using two different models. In the first model, risk-neutral bidders submit bids in serial and try to surpass a stochastic seller threshold while taking into account how many previous failed bids were made by other bidders. We compute optimal strategies for this model and show that bidder expected surplus decreases in the number of previous failed bids. In the second model we assume bidders do not know how many previous failed bids have been made, and instead use a first-price sealed-bid mechanism with a buy-out price where bidders serially submit bids with the knowledge that no previous bidders have used the buy-out price. We derive a unique equilibrium bidding strategy for risk-neutral bidders in this serial model, show that any equilibrium in a similar parallel bidding model is the same as the equilibrium in the serial model, and compute seller revenue. In particular, under certain circumstances, bidders will bid more in this format than they would in a standard first-price sealed-bid auction, but that a seller maximizes expected revenue by setting a buy-out price higher than any bidder is willing to pay thereby making the auction essentially a first-price auction.
\end{abstract}

\section{KEYWORDS}

Auction Theory; eBay; Buy-It-Now or Best Offer; Symmetric Bayesian Nash Equilibrium; Buy-Out Price; First-Price SealedBid

\section{DEDICATION}

We dedicate this paper to our coauthor and friend Jamie Oliva (1994-2016).

\section{INTRODUCTION}

eBay is one of the internet's largest hubs of person-to-person commerce. The website hosts online auctions where sellers can sell goods directly to consumers and has been extensively studied by economists. ${ }^{1}$ The standard auction on eBay, dubbed by some as a California auction, ${ }^{2}$ is a timed auction where bidders may submit bids at any time. A bidder submits a maximum amount he or she $\mathrm{b}^{\mathrm{b}}$ is willing to pay for the item, and any time another bidder bids an amount less that that maximum amount, eBay will bid on behalf of that original bidder the smallest possible bid needed to exceed the competing bid (eBay calls this system "proxy bidding"). In 2000 eBay also allowed sellers to include a "Buy-It-Now" price on these auctions, where any bidder could end the auction early by bidding the fixed "Buy-It-Now" price (abbreviated as BIN). This California auction mechanism is well-studied, and Reynolds and Wooders studied the effect of the inclusion of a "Buy-It-Now" price in that type of auction. ${ }^{3}$ In this paper we consider another selling mechanism on eBay. eBay offers a format where a seller can list an item at a fixed "Buy-It-Now" price but has the option to consider offers from buyers for less than that price. eBay labels such an auction as a Buy-It-Now auction with Best Offer option, but we will refer to such auctions as simply Best Offer auctions. Instead of a base format of an ascending English auction as in the California auction, the Best Offer auction is a close cousin of a first-price sealed-bid auction. In such an auction, bidders secretly submit offers to the seller and the auction winner has to pay her offer. The Best Offer auction is different than a standard first-price sealed-bid auction in that there is a

\footnotetext{
${ }^{a}$ This research was supported by funds from a National Science Foundation grant (\#DMS1148695) through the Center for Undergraduate Research in Mathematics (CURM), Brigham Young University, and sponsors.

${ }^{\mathrm{b}}$ In the future when a generic pronoun is called for, the authors will default to the feminine.
} 
buy-out price (the BIN price) that a bidder can submit and win the item with certainty. For an example of such an auction, see Figure 1. The aim of this paper is to mathematically model optimal strategies for bidding in such Best Offer auctions.

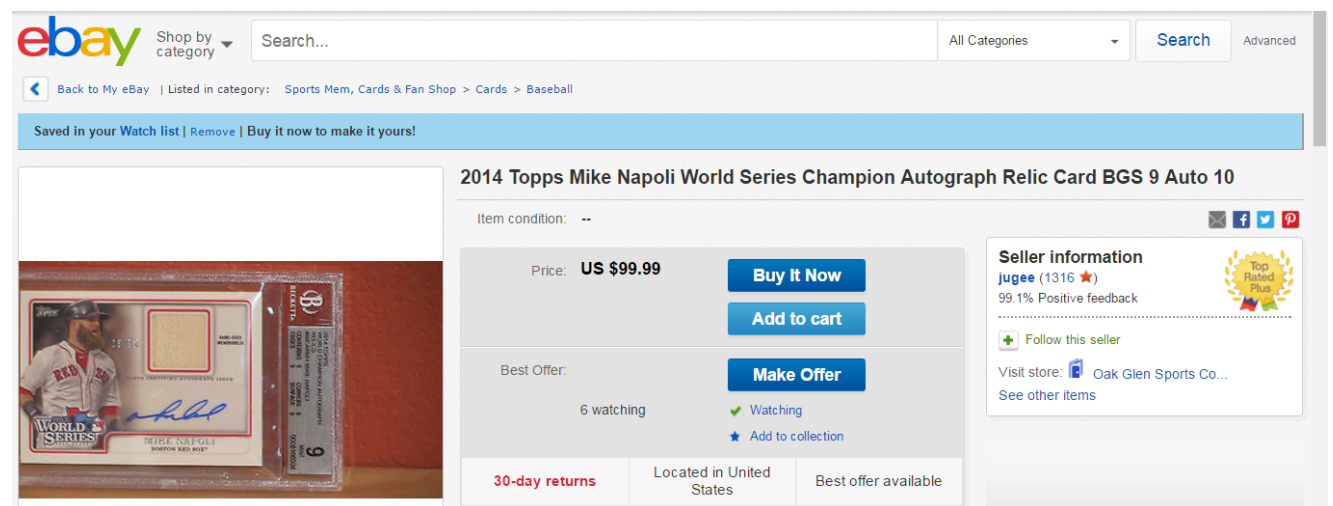

Figure 1. An example of a Best Offer auction page on eBay. Note the options to either "Buy It Now" or "Make an Offer."

When deciding to place a bid (either an offer or the BIN), a bidder can take into consideration other pieces of information that could influence her bid. In the past, eBay would include the number of previous bids made (but not the amount of those bids). This information included whether or not the offer was "pending" (that is, still under consideration by the seller) or "declined." In our first model of the Best Offer auction, which we will call the Secret Threshold game or just ST game, we assume every bidder knows how many previous bids the seller has declined. We exclude the number of pending bids to simplify the model, and to isolate the impact of the number of previous failed bids on optimal bidding behavior. The investigation of this model is the subject of the first section of the paper.

Several years ago, eBay changed the type of information available to prospective bidders. No longer is the number of previous offers (both pending or declined) public. Instead, now the number of "watchers" is public. On eBay, users can "watch" an auction of any format. This means that the user selects the auction to be saved as a part of that user's personal "watch list." However, it is not entirely clear how much useful knowledge a bidder can glean from the number of users watching a Best Offer auction, as watchers need not be past nor future bidders. To account for this uncertainty, we will model the Best Offer auction as a first-price sealed-bid auction with a buy-out price where the number of bidders is unknown but satisfies some given probability distribution. The unknown bidder structure we use is akin to the model developed by McAfee and McMillan. ${ }^{4}$

Generally, the strategies for a first-price sealed-bid auction with an unknown number of bidders is known, ${ }^{4,5}$ however our main model for this scenario, which we dub the Serial First Price Buy-Out (SFPBO) auction, has two other wrinkles. The first wrinkle, as previously stated, is that there is a buy-out price, and thus there should be a threshold value over which a bidder will decide to use the buy-out price instead of submitting a bid. The second wrinkle is that we assume that bidders will place bids in serial in a randomly prescribed but unknown order. Generally in a first-price sealed-bid auction, bidders are modeled submitting bids simultaneously. However, in practice on eBay, bidders will see and bid on an Best Offer auction at different times. The mere fact that a Best Offer auction has not yet ended gives a bidder some information. Specifically, the bidder in this case knows that any previous bidders have submitted offers lower than the BIN. Our model will account for this serial nature of bid submission and this extra information available to bidders.

In the second section of the paper, we derive an equilibrium strategy for risk-neutral bidders for the SFPBO auction. In the third section we compare this equilibrium to an equilibrium to a more standard first-price with buy-out model which we will dub the First Price Buy-Out (FPBO) auction where bids are submitted simultaneously, and show that any equilibrium for the FPBO auction must be the same as the equilibrium for the SFPBO auction. Finally, in the final section, we will see that, in equilibrium a seller maximizes revenue by setting the buy-out price higher than any risk-neutral bidder is willing to 
pay. Thus, a seller in this setting does best by making the auction effectively a first-price sealed-bid auction without a reserve price.

\section{THE SECRET THRESHOLD GAME}

We start by considering a model, which we will call the Secret Threshold (ST) game, where bidders will know how many previous failed offers have been placed on the item up for bid. One important simplification we are making here (and as well in our next model) is that we are assuming each bidder can submit only one offer for the item. On eBay, buyers can submit up to three offers, and sellers have the option to make counteroffers on the first two offers. We do not include this possibility in our model as we are not interested in studying negotiation strategy, but the effect of the knowledge of previously failed bids on optimal bid making. Note that in any event, if the buyer makes three offers, the last offer is necessarily a final take-itor-leave-it offer, which matches our model. On eBay, even if the seller declines an offer, the buyer can still purchase the item through the Buy-It-Now option. In our model we do not include such an option, as we are more interested in how a bidder crafts a single optimal offer free of negotiation. However, our model below could be modified to allow for this option.

We call this model an ST game because in this model bidders are trying to exceed (by as thin of as margin possible) an unknown stochastic threshold set by a seller. While we use the generic term of "threshold" here, in auction theoretic terms this threshold is just the seller's reservation price. We call this a "game" rather than an "auction" since bidders do not directly compete against one another in this model. Again, this is a simplification as eBay gives sellers 48 hours to respond to an offer, and so certainly within that time frame a seller could have multiple "pending" offers. However, in this model, we are less concerned with the effects of competition among the bidders and more on the effects of bidders knowing the number of failed bids. With that said, below we give the mathematical assumptions of the ST game.

Definition 1. The conditions of Secret Threshold (ST) game are:

(i) There are a countable number of bidders. For $n \in \mathbb{N}$, the $n^{\text {th }}$ bidder's value is the random variable $V_{n}$ with range $[0,1]$ and has a cumulative distribution function ( $c d f) F(v)$ and a probability density function ( $p d f) f(v)$. Note that this means all bidder values are identically distributed.

(ii) The $i^{\text {th }}$ bidder knows her own value $v_{i}$ for the item and how many failed bids have been previously made. That is, the first bidder knows she is the first bidder and if $n>1$, the $n^{\text {th }}$ bidder knows there have been $n-1$ previous failed bids. The $n^{\text {th }}$ bidder does not know the values of $V_{i}$ for $i \neq n$, but does know they have followed the distribution $F(v)$.

(iii) There is a value $0<\omega \leq 1$, called the buy-out price, at which the seller is guaranteed to sell the item. Furthermore, there is a random variable $B_{0}$ with range of $[0, \omega]$ with the $c d f H(b)$ and $p d f h(b)$. The seller will accept any offer that exceeds $B_{0}$ (that is, $B_{0}$ represents the "secret threshold" of this game). That is, if the $n^{\text {th }}$ bidder submits the bid $b$, if $b \geq B_{0}$ then this bidder wins the auction and gains the surplus $v_{n}-b$. If not, the bidder does not win and has a surplus of 0 . That is, the surplus function, $\Pi^{S T}$, is given by

$$
\Pi^{S T}\left(v_{n}, b\right)=\left(v_{n}-b\right) \mathbb{1}_{b \geq B_{0}},
$$

Equation 1.

where $\mathbb{1}_{b \geq B_{0}}$ is the indicator random variable which equals 1 when $b \geq B_{0}$ and equals 0 when $b<B_{0}$.

(iv) The random variables $B_{0}, V_{1}, V_{2}, V_{3}, \ldots$ are all independent.

(v) The pdf $h(b)$ is positive and continuous on $[0, \omega]$ and the $p d f f(v)$ is positive and continuous on $(0,1)$.

The last two assumptions above are technical mathematical impositions to simplify the model. In particular, one could assume some positive correlation between the bidder values $V_{1}, V_{2}, \ldots$ (often in auction theory one assumes that values are affiliated ${ }^{2,5,6}$ ) but we will keep things simpler here. Our goal here is to find a bid that will maximize the expected surplus for the $n^{\text {th }}$ bidder given that they know there have been $n-1$ failed bids before them. That is, for bidder 1 , we want a bid $b_{1}\left(v_{1}\right)$ that will maximize $E\left[\Pi^{S T}\right]$. For any bidder $n>1$, we want to maximize the conditional expected value 
$E\left[\Pi^{S T} \mid \max _{1 \leq i \leq n-1} b_{i}\left(V_{i}\right)<B_{0}\right]$. That is, the $n^{\text {th }}$ bidder assumes the previous bidders bid optimally and still lost, and thus conditions her expectation on that fact. As is suggested by the previous discussion, we will inductively create these bidding functions $b_{n}:[0,1] \rightarrow[0, \omega]$.

\section{The First Bidder}

First we will consider the first bidder in a ST game. Our bidder would like to find a bid $b_{1}$ that maximizes her expected surplus given that her value is $v_{1}$. Note that by assumption (iii) of the ST game, we have

$$
E\left[\Pi^{S T}\left(v_{1}, b_{1}\right)\right]=\left(v_{1}-b_{1}\right) P\left(b_{1} \geq B_{0}\right)=\left(v_{1}-b_{1}\right) H\left(b_{1}\right) .
$$

Equation 2.

In an attempt to maximize the above, we take the derivative with respect to $b_{1}$ and set the resulting quantity to 0 to look for critical points:

$$
0=\frac{d}{d b_{1}}\left(E\left[\Pi^{S T}\right]\right)=-H\left(b_{1}\right)+\left(v_{1}-b_{1}\right) h\left(b_{1}\right) .
$$

Equation 3.

Solving the above for $v_{1}$ gives us

$$
v_{1}=b_{1}+\frac{H\left(b_{1}\right)}{h\left(b_{1}\right)} .
$$

Equation 4.

The above equation motivates the following definition.

Definition 2. Assume the conditions of the ST game. Define a function $\phi_{1}:[0, \omega] \rightarrow[0, \infty)$ as

$$
\phi_{1}(x)=x+\frac{H(x)}{h(x)} .
$$

Equation 5.

The function $\phi_{1}$ is very similar but not equal to to the marginal revenue function $\operatorname{MR}(x)=x-\frac{1-H(x)}{h(x)}$ often cited in the Revenue Equivalence Theorem. Further, we note that $\phi_{1}$ is continuous.

Proposition 3. The function $\phi_{1}$ defined in Definition 2 is continuous on $[0,1]$.

Proof. Note that since $h$ is continuous on $[0,1]$ and positive by assumption (v), then $\phi_{1}$ is also continuous on $[0,1]$.

We can now state our first theorem.

Theorem 4. Assume the conditions of the ST game and suppose the function $\phi_{1}(b)=b+\frac{H(b)}{h(b)}$ is increasing on $[0, \omega]$. The bidding function $b_{1}:[0,1] \rightarrow[0, \omega]$ that uniquely maximizes the expected surplus $E\left[\Pi^{S T}\right]$ is

$$
b_{1}(v)=\left\{\begin{array}{ll}
\phi_{1}^{-1}(v) & \text { if } v \leq \phi_{1}(\omega) \\
\omega & \text { else }
\end{array} .\right.
$$

Equation 6.

Note that the above bidding strategy has a nice form. It tells us exactly when to use the BIN $\omega$ instead of sending an offer (when $v \leq \phi_{1}(\omega)$ ). Also, in the above theorem we assume that $\phi_{1}$ is increasing. This is akin to the simplifying (but not necessary) assumption found in Myerson that the marginal revenue function MR is increasing when finding an optimal auction mechanism. ${ }^{7}$

Proof. By the previous proposition, $\phi_{1}$ is continuous. As $\phi_{1}$ is also assumed to be increasing on its domain, it is invertible and we have $\phi_{1}^{-1}:\left[\phi_{1}(0), \phi(\omega)\right] \rightarrow[0, \omega]$. In fact, evaluating $\phi_{1}$ at 0 yields $\phi_{1}(0)=0+0=0$. So, we have $\phi_{1}^{-1}:[0, \phi(\omega)] \rightarrow$ $[0, \omega]$. We have two cases to consider: the case where $v_{1} \leq \phi(\omega)$ and the case where $v_{1}>\phi(\omega)$. First, suppose $v_{1} \leq \phi_{1}(\omega)$ so that $\phi_{1}^{-1}\left(v_{1}\right)$ is defined. If $b_{1}<\phi_{1}^{-1}\left(v_{1}\right)$, then $\phi_{1}\left(b_{1}\right)<v_{1}$ and thus,

$$
\frac{d}{d b_{1}}\left(E\left[\Pi^{S T}\right]\right)=-H\left(b_{1}\right)+\left(v_{1}-b_{1}\right) h\left(b_{1}\right)>-H\left(b_{1}\right)+\left(\phi_{1}\left(b_{1}\right)-b_{1}\right) h\left(b_{1}\right)=0 .
$$

Equation 7. 
This shows that $E\left[\Pi^{S T}\right]$ is increasing for $b_{1}<\phi_{1}^{-1}\left(v_{1}\right)$. On the other hand, if $b_{1}>\phi_{1}^{-1}\left(v_{1}\right)$, then $\phi_{1}\left(b_{1}\right)>v$ and thus

$$
\frac{d}{d b_{1}}\left(E\left[\Pi^{S T}\right]\right)=-H\left(b_{1}\right)+\left(v_{1}-b_{1}\right) h\left(b_{1}\right)<-H\left(b_{1}\right)+\left(\phi_{1}\left(b_{1}\right)-b_{1}\right) h\left(b_{1}\right)=0
$$

Equation 8.

showing that $E\left[\Pi^{S T}\right]$ is decreasing for $b_{1}<\phi_{1}^{-1}\left(v_{1}\right)$. Thus, $b_{1}=\phi_{1}^{-1}\left(v_{1}\right)$ is the bid that maximizes the expected surplus $E\left[\Pi^{S T}\right]$ when $v_{1} \leq \phi_{1}(\omega)$.

Next, suppose $v_{1}>\phi_{1}(\omega)$. Then we have $v_{1}>\phi_{1}(\omega) \geq \phi_{1}\left(b_{1}\right)$ for any $b_{1} \leq \omega$. So

$$
\frac{d}{d b_{1}}\left(E\left[\Pi^{S T}\right]\right)=-H\left(b_{1}\right)+\left(v_{1}-b_{1}\right) h\left(b_{1}\right)>-H\left(b_{1}\right)+\left(\phi_{1}\left(b_{1}\right)-b_{1}\right) h\left(b_{1}\right)=0 .
$$

Equation 9.

So $E\left[\Pi^{S T}\right]$ is increasing for all bids $b_{1}$. Thus, the quantity is maximized at the maximum bid $\omega$.

For an example, suppose the secret reserve $B_{0}$ has a uniform distribution on $[0, \omega]$. Then $H(b)=\frac{1}{\omega} b$ and $h(b)=\frac{1}{\omega}$, giving us $\phi_{1}(b)=2 b$ and thus $\phi_{1}^{-1}(v)=\frac{1}{2} v$. Thus,

$$
b_{1}(v)= \begin{cases}\frac{1}{2} v & \text { if } v \leq 2 \omega \\ \omega & \text { else. }\end{cases}
$$

Equation 10.

\section{Bidding After Failed Bids}

Now let's consider the strategy for the $n^{\text {th }}$ bidder where $n>1$. The $n^{\text {th }}$ bidder is bidding after $n-1$ failed bids and thus wants to maximize the conditional expected value

$$
E\left[\Pi^{S T}\left(v_{n}, b\right) \mid \max _{1 \leq i \leq n-1} b_{i}\left(V_{i}\right) \leq B_{0}\right]
$$

Equation 11.

in $b$. In the theorem below, we establish an inductive formula for constructing an optimal strategy for the $n^{\text {th }}$ bidder.

Theorem 5. Let $n>1$ and assume the conditions of the ST game. Suppose for $1 \leq i \leq n-1$ there are bidding functions $b_{i}$ defined for the $i^{\text {th }}$ bidder in a ST game of the form

$$
b_{i}(v)=\left\{\begin{array}{ll}
\phi_{i}^{-1}(v) & \text { if } v \leq \phi_{i}(\omega) \\
\omega & \text { else }
\end{array},\right.
$$

where $\phi_{i}$ is continuous and increasing on $[0, \omega]$ and $\phi_{i}(0)=0$. Define the function $\phi_{n}(b)$ as

$$
\phi_{n}(b):=\left\{\begin{array}{cc}
b+\frac{\int_{0}^{b}\left(\prod_{i=1}^{n-1} F\left(\phi_{i}\left(b_{0}\right)\right)\right) h\left(b_{0}\right) d b_{0}}{\left(\prod_{i=1}^{n-1} F\left(\phi_{i}(b)\right)\right) h(b)} & \text { if } b>0 \\
0 & \text { if } b=0
\end{array} .\right.
$$

Then $\phi_{n}(b)$ is continuous. Furthermore, if $\phi_{n}(b)$ is increasing, the function $b_{n}(v)$ defined as

$$
b_{n}(v)= \begin{cases}\phi_{n}^{-1}(v) & \text { if } v \leq \phi_{n}(\omega) \\ \omega & \text { else }\end{cases}
$$

Equation 14.

uniquely maximizes the expected surplus $E\left[\Pi^{S T} \mid \max _{1 \leq i \leq n-1} b_{i}\left(V_{i}\right) \leq B_{0}\right]$ of the $n^{\text {th }}$ bidder in a ST game.

Proof. First, note that the function $\phi_{n}$ defined in Equation 13 is well-defined for $b>0$ by the fact that each $\phi_{i}$ is increasing, $\phi_{i}(0)=0$, and by assumption (v) of the ST game. Since each $\phi_{i}$ is continuous and $h$ and $F$ are continuous by assumption 
(v), $\phi_{n}$ is continuous on $(0, \omega]$. To check continuity at $b=0$, note that since $F\left(\phi_{i}(b)\right)$ is increasing, we have

$$
\begin{aligned}
0 & \leq \phi_{n}(b)=b+\frac{\int_{0}^{b}\left(\prod_{i=1}^{n-1} F\left(\phi_{i}\left(b_{0}\right)\right)\right) h\left(b_{0}\right) d b_{0}}{\left(\prod_{i=1}^{n-1} F\left(\phi_{i}(b)\right)\right) h(b)} \\
& <b+\frac{\int_{0}^{b}\left(\prod_{i=1}^{n-1} F\left(\phi_{i}(b)\right)\right) h\left(b_{0}\right) d b_{0}}{\left(\prod_{i=1}^{n-1} F\left(\phi_{i}(b)\right)\right) h(b)}=b+\phi_{1}(b),
\end{aligned}
$$

Equation 15.

showing (by the Squeeze Theorem) that $\phi_{n}(b) \rightarrow 0$ as $b \rightarrow 0^{+}$. Thus, $\phi_{n}$ is continuous on its domain.

To prove the second part of the theorem, we assume that $\phi_{n}$ is also increasing. For any bid $b_{n}$, we have

$$
E\left[\Pi^{S T}\left(v_{n}, b_{n}\right) \mid \max _{1 \leq i \leq n-1} b_{i}\left(V_{i}\right) \leq B_{0}\right]=\left(v_{n}-b_{n}\right) P\left(B_{0} \leq b_{n} \mid b_{1}\left(V_{1}\right) \leq B_{0}, \ldots, b_{n-1}\left(V_{n-1}\right) \leq B_{0}\right) . \quad \text { Equation } 16 .
$$

Note that if $b_{i}\left(V_{i}\right)<B_{0}$, we know that bidder $i$ did not bid the BIN, $\omega$, meaning she must have bid $\phi_{i}^{-1}\left(V_{i}\right)$. By assumptions (i) and (iv) the values $V_{i}$ are iid with $\operatorname{cdf} F(v)$ and pdf $f(v)$ and the random variables $B_{0}, V_{1}, \ldots, V_{n-1}$ are all independent. Thus we have

$$
\begin{aligned}
P\left(b_{1}\left(V_{1}\right) \leq B_{0}, \ldots, b_{n-1}\left(V_{n-1}\right) \leq B_{0}\right) & =P\left(V_{1} \leq \phi_{1}\left(B_{0}\right), \ldots, V_{n-1} \leq \phi_{n-1}\left(B_{0}\right)\right) \\
& =\int_{0}^{\omega} \int_{0}^{\phi_{1}\left(b_{0}\right)} \ldots \int_{0}^{\phi_{n-1}\left(b_{0}\right)} h\left(b_{0}\right) f\left(v_{1}\right) \ldots f\left(v_{n-1}\right) d v_{n-1} \ldots d v_{1} d b_{0} \\
& =\int_{0}^{\omega}\left(\prod_{i=1}^{n-1} \int_{0}^{\phi_{i}\left(b_{0}\right)} f\left(v_{i}\right) d v_{i}\right) h\left(b_{0}\right) d b_{0}=\int_{0}^{\omega}\left(\prod_{i=1}^{n-1} F\left(\phi_{i}\left(b_{0}\right)\right)\right) h\left(b_{0}\right) d b_{0} .
\end{aligned}
$$

Equation 17.

Similarly, we can compute

$$
P\left(B_{0} \leq b, b_{1}\left(V_{1}\right) \leq B_{0}, \ldots, b_{n-1}\left(V_{n-1}\right) \leq B_{0}\right)=\int_{0}^{b}\left(\prod_{i=1}^{n-1} F\left(\phi_{i}\left(b_{0}\right)\right)\right) h\left(b_{0}\right) d b_{0} .
$$

Equation 18.

Inserting Equation 17 and Equation 18 into Equation 16 yields

$$
E\left[\Pi^{S T}\left(v_{n}, b\right) \mid \max _{1 \leq i \leq n-1} b_{i}\left(V_{i}\right) \leq B_{0}\right]=\left(v_{n}-b\right) \frac{\int_{0}^{b}\left(\prod_{i=1}^{n-1} F\left(\phi_{i}\left(b_{0}\right)\right)\right) h\left(b_{0}\right) d b_{0}}{\int_{0}^{\omega}\left(\prod_{i=1}^{n-1} F\left(\phi_{i}\left(b_{0}\right)\right)\right) h\left(b_{0}\right) d b_{0}} .
$$

Equation 19.

Thus we can compute the derivative of $E\left[\Pi^{S T}\left(v_{n}, b\right) \mid \max _{1 \leq i \leq n-1} b_{i}\left(V_{i}\right) \leq B_{0}\right]$ :

$$
\frac{d}{d b} E\left[\Pi^{S T}\left(v_{n}, b\right) \mid \max _{1 \leq i \leq n-1} b_{i}\left(V_{i}\right) \leq B_{0}\right]=\frac{-\int_{0}^{b}\left(\prod_{i=1}^{n-1} F\left(\phi_{i}\left(b_{0}\right)\right)\right) h\left(b_{0}\right) d b_{0}+(v-b)\left(\prod_{i=1}^{n-1} F\left(\phi_{i}(b)\right)\right) h(b)}{\int_{0}^{\omega}\left(\prod_{i=1}^{n-1} F\left(\phi_{i}\left(b_{0}\right)\right)\right) h\left(b_{0}\right) d b_{0}} .
$$

Equation 20.

Suppose that $v_{n} \leq \phi_{n}(\omega)$. Then $v_{n}$ is in the range of $\phi_{n}$ so we can write $\phi_{n}^{-1}\left(v_{n}\right)$. Next suppose $b<\phi_{n}^{-1}\left(v_{n}\right)$. Since $\phi_{n}$ is increasing, we have $\phi_{n}(b)<v_{n}$, so

$$
\begin{aligned}
\frac{d}{d b} E\left[\Pi^{S T}\left(v_{n}, b\right) \mid \max _{1 \leq i \leq n-1} b_{i}\left(V_{i}\right) \leq B_{0}\right] & =\frac{-\int_{0}^{b}\left(\prod_{i=1}^{n-1} F\left(\phi_{i}\left(b_{0}\right)\right)\right) h\left(b_{0}\right) d b_{0}+\left(v_{n}-b\right)\left(\prod_{i=1}^{n-1} F\left(\phi_{i}(b)\right)\right) h(b)}{\int_{0}^{\omega}\left(\prod_{i=1}^{n-1} F\left(\phi_{i}\left(b_{0}\right)\right)\right) h\left(b_{0}\right) d b_{0}} \\
& >\frac{-\int_{0}^{b}\left(\prod_{i=1}^{n-1} F\left(\phi_{i}\left(b_{0}\right)\right)\right) h\left(b_{0}\right) d b_{0}+\left(\phi_{n}(b)-b\right)\left(\prod_{i=1}^{n-1} F\left(\phi_{i}(b)\right)\right) h(b)}{\int_{0}^{\omega}\left(\prod_{i=1}^{n-1} F\left(\phi_{i}\left(b_{0}\right)\right)\right) h\left(b_{0}\right) d b_{0}} \\
& =0,
\end{aligned}
$$


so that $E\left[\Pi^{S T}\left(v_{n}, b\right) \mid \max _{1 \leq i \leq n-1} b_{i}\left(V_{i}\right) \leq B_{0}\right]$ is increasing for $b<\phi_{n}^{-1}\left(v_{n}\right)$. Now suppose $b>\phi_{n}^{-1}\left(v_{n}\right)$. Since $\phi_{n}$ is increasing, we have $\phi_{n}(b)>v_{n}$, so

$$
\begin{aligned}
\frac{d}{d b} E\left[\Pi^{S T}\left(v_{n}, b\right) \mid \max _{1 \leq i \leq n-1} b_{i}\left(V_{i}\right) \leq B_{0}\right] & =\frac{-\int_{0}^{b}\left(\prod_{i=1}^{n-1} F\left(\phi_{i}\left(b_{0}\right)\right)\right) h\left(b_{0}\right) d b_{0}+\left(v_{n}-b\right)\left(\prod_{i=1}^{n-1} F\left(\phi_{i}(b)\right)\right) h(b)}{\int_{0}^{\omega}\left(\prod_{i=1}^{n-1} F\left(\phi_{i}\left(b_{0}\right)\right)\right) h\left(b_{0}\right) d b_{0}} \\
& <\frac{-\int_{0}^{b}\left(\prod_{i=1}^{n-1} F\left(\phi_{i}\left(b_{0}\right)\right)\right) h\left(b_{0}\right) d b_{0}+\left(\phi_{n}(b)-b\right)\left(\prod_{i=1}^{n-1} F\left(\phi_{i}(b)\right)\right) h(b)}{\int_{0}^{\omega}\left(\prod_{i=1}^{n-1} F\left(\phi_{i}\left(b_{0}\right)\right)\right) h\left(b_{0}\right) d b_{0}} \\
& =0, \quad \text { Equation } 22 .
\end{aligned}
$$

so that $E\left[\Pi^{S T}\left(v_{n}, b\right) \mid \max _{1 \leq i \leq n-1} b_{i}\left(V_{i}\right) \leq B_{0}\right]$ is decreasing for $b<\phi_{n}^{-1}\left(v_{n}\right)$. Thus, $E\left[\Pi^{S T}\left(v_{n}, b\right)\right]$ is maximized at $b=\phi_{n}^{-1}\left(v_{n}\right)$ if $v_{n} \leq \phi_{n}(\omega)$.

Next we consider the case where $v_{n}>\phi_{n}(\omega)$. In this case, for any $b<\omega$ we have $\phi_{n}(b)<\phi_{n}(\omega)<v_{n}$, so

$$
\begin{aligned}
& \frac{d}{d b} E\left[\Pi^{S T}\left(v_{n}, b\right) \mid \max _{1 \leq i \leq n-1} b_{i}\left(V_{i}\right) \leq B_{0}\right] \\
& =\frac{-\int_{0}^{b}\left(\prod_{i=1}^{n-1} F\left(\phi_{i}\left(b_{0}\right)\right)\right) h\left(b_{0}\right) d b_{0}+\left(v_{n}-b\right)\left(\prod_{i=1}^{n-1} F\left(\phi_{i}(b)\right)\right) h(b)}{\int_{0}^{\omega}\left(\prod_{i=1}^{n-1} F\left(\phi_{i}\left(b_{0}\right)\right)\right) h\left(b_{0}\right) d b_{0}} \\
& >\frac{-\int_{0}^{b}\left(\prod_{i=1}^{n-1} F\left(\phi_{i}\left(b_{0}\right)\right)\right) h\left(b_{0}\right) d b_{0}+\left(\phi_{n}(b)-b\right)\left(\prod_{i=1}^{n-1} F\left(\phi_{i}(b)\right)\right) h(b)}{\int_{0}^{\omega}\left(\prod_{i=1}^{n-1} F\left(\phi_{i}\left(b_{0}\right)\right)\right) h\left(b_{0}\right) d b_{0}}=0,
\end{aligned}
$$

Equation 23.

showing that $E\left[\Pi^{S T}\left(v_{n}, b\right) \mid \max _{1 \leq i \leq n-1} b_{i}\left(V_{i}\right) \leq B_{0}\right]$ is always increasing, and thus is maximized on the right endpoint $\omega$, proving the theorem.

The above theorem gives an inductive way to find the bidding functions of each bidder. Note that in the statement of Theorem 5, we actually do not assert that for $1 \leq i \leq n-1$ the strategy $b_{i}\left(v_{i}\right)$ maximizes the conditional expected surplus for bidder $i$, but only that $b_{i}$ satisfies Equation 12. However, assuming rational bidders, the $n^{\text {th }}$ bidder could assume all previous bidders bid optimally, so when we construct examples of these functions below, we will assume all bidders have used the optimal bidding function.

For an example, let's suppose the seller's reserve $B_{0}$ has a uniform distribution on $[0, \omega]$ and the other bidders distributions are also uniform over $[0,1]$. We will inductively show that the maximizing bidding function is

$$
b_{n}(v)=\left\{\begin{array}{ll}
\left(1-\frac{1}{n+1}\right)(v) & \text { if } v \leq \frac{n+1}{n} \omega \\
\omega & \text { else. }
\end{array} .\right.
$$

Equation 24.

Note that in the base case, we have previously shown that

$$
b_{1}(v)= \begin{cases}\left(1-\frac{1}{2}\right) v & \text { if } v \leq 2 \omega \\ \omega & \text { else }\end{cases}
$$

which fits our formula when $n=1$. For the inductive step, let $n>1$ and assume the formula holds for all $i$ such that $1 \leq i \leq n-1$. Thus, for $1 \leq i \leq n-1$

$$
b_{i}(v)=\left\{\begin{array}{ll}
\left(1-\frac{1}{i+1}\right)(v) & \text { if } v \leq \frac{i+1}{i}(\omega) \\
\omega & \text { else }
\end{array} .\right.
$$

Equation 26. 
Note that by the above formula, and by Theorem $\mathbf{5}$ we have $\phi_{i}(b)=\frac{i+1}{i} b$. Also by Theorem $\mathbf{5}$ we have

$$
\begin{aligned}
\phi_{n}(b) & =b+\frac{\int_{0}^{b}\left(\prod_{i=1}^{n-1} F\left(\phi_{i}\left(b_{0}\right)\right)\right) h\left(b_{0}\right) d b_{0}}{\left(\prod_{i=1}^{n-1} F\left(\phi_{i}(b)\right)\right) h(b)} \\
& =b+\frac{\int_{0}^{b}\left(\prod_{i=1}^{n-1} F\left(\frac{i+1}{i}\left(b_{0}\right)\right)\right) \frac{1}{\omega} d b_{0}}{\left(\prod_{i=1}^{n-1} F\left(\frac{i+1}{i}(b)\right)\right) \frac{1}{\omega}} \\
& =b+\frac{\int_{0}^{b}\left(\prod_{i=1}^{n-1} \frac{i+1}{i}\left(b_{0}\right)\right) d b_{0}}{\left(\prod_{i=1}^{n-1} \frac{i+1}{i}(b)\right)} \\
& =b+\frac{\int_{0}^{b}\left(b_{0}^{n-1}\right) d b_{0}}{\left(b^{n-1}\right)} \\
& =b+\frac{b^{n}}{\left(b^{n-1}\right)} \\
& =b+\frac{b}{n} \\
& =\frac{n+1}{n} b .
\end{aligned}
$$

Equation 27.

Thus we have $\phi_{n}^{-1}(v)=\frac{n}{n+1} v=\left(1-\frac{1}{n+1}\right) v$, and thus

$$
b_{n}(v)=\left\{\begin{array}{ll}
\left(1-\frac{1}{n+1}\right)(v) & \text { if } v \leq \frac{n+1}{n}(\omega) \\
\omega & \text { else }
\end{array},\right.
$$

Equation 28.

completing the induction.

\section{Ordering of The Bidding Functions}

One might deduce from the uniform example above that bidding functions $b_{n}(v)$ generally increase as $n$ increases. Generally one would suspect that bidders failing to win the item is a signal to subsequent bidders that the secret reserve is high and such bidders should be more aggressive in their bids. This is indeed the case.

Theorem 6. Let $n>1$. Suppose the conditions of the ST game and of Theorem 5 are satisfied and the functions $b_{n-1}$ and $b_{n}$ are defined as in Theorem 5. If $v=0$ or $v \geq \phi_{n-1}(\omega)$ we have

$$
b_{n-1}(v)=b_{n}(v) .
$$

Equation 29.

If $0<v<\phi_{n-1}(\omega)$, then

$$
b_{n-1}(v)<b_{n}(v) \text {. }
$$

Equation 30.

Proof. Note that $b_{n-1}(0)=0=b_{n}(0)$. Note that for $b>0$, if we set the product $\prod_{i=1}^{0} F\left(\phi_{i}(b)\right)$ to equal 1 to cover the case where $n=2$, we have

$$
\begin{aligned}
\phi_{n-1}(b) & =b+\frac{\int_{0}^{b}\left(\prod_{i=1}^{n-2} F\left(\phi_{i}\left(b_{0}\right)\right)\right) h\left(b_{0}\right) d b_{0}}{\left(\prod_{i=1}^{n-2} F\left(\phi_{i}(b)\right)\right) h(b)} \\
& =b+\frac{\int_{0}^{b}\left(\prod_{i=1}^{n-2} F\left(\phi_{i}\left(b_{0}\right)\right)\right) F\left(\phi_{n-1}(b)\right) h\left(b_{0}\right) d b_{0}}{\left(\prod_{i=1}^{n-1} F\left(\phi_{i}(b)\right)\right) h(b)} \\
& >b+\frac{\int_{0}^{b}\left(\prod_{i=1}^{n-1} F\left(\phi_{i}\left(b_{0}\right)\right)\right) h\left(b_{0}\right) d b_{0}}{\left(\prod_{i=1}^{n-1} F\left(\phi_{i}(b)\right)\right) h(b)}=\phi_{n}(b),
\end{aligned}
$$

Equation 31. 
where the inequality is justified by the fact that $F\left(\phi_{n-1}\left(b_{0}\right)\right)$ is an increasing function. We will repeatedly use the fact $\phi_{n-1}(b)>\phi_{n}(b)$ for $b>0$ in what follows.

Suppose that $v \geq \phi_{n-1}(\omega)$. Then by the above we also have $v>\phi_{n}(\omega)$. Thus, we have

$$
b_{n-1}(v)=\omega=b_{n}(v) .
$$

Equation 32.

We next will show that for all other values of $v$ (i.e. $0<v<\phi_{n-1}(\omega)$ ) we have the strict inequality $b_{n-1}(v)<b_{n}(v)$. Suppose that $\phi_{n}(\omega) \leq v<\phi_{n-1}(\omega)$. Note that $b_{n}(v)=\omega$. Also, since $\phi_{n-1}$ is increasing we have

$$
b_{n-1}(v)=\phi_{n-1}^{-1}(v)<\phi_{n-1}^{-1}\left(\phi_{n-1}(\omega)\right)=\omega=b_{n}(v) .
$$

Equation 33.

Lastly, suppose $0<v<\phi_{n}(\omega)$. Then $b_{n-1}(v)=\phi_{n-1}^{-1}(v)<\omega$ and $b_{n}(v)=\phi_{n}^{-1}(v)<\omega$. First note that

$$
v=\phi_{n}\left(\phi_{n}^{-1}(v)\right)<\phi_{n-1}\left(\phi_{n}^{-1}(v)\right) .
$$

Equation 34.

Using the above, we have

$$
b_{n-1}(v)=\phi_{n-1}^{-1}(v)<\phi_{n-1}^{-1}\left(\phi_{n-1}\left(\phi_{n}^{-1}(v)\right)\right)=\phi_{n}^{-1}(v)=b_{n}(v),
$$

Equation 35.

completing the proof.

This increase of bids affects the conditional expected surplus. This surplus can also be affected by the conditional probability in the $n-1^{\text {th }}$ and $n^{\text {th }}$ cases. In particular, one can show that for any fixed bid $b \leq \omega$, we have

$$
P\left(B_{0} \leq b \mid b_{1}\left(V_{1}\right) \leq B_{0}, \ldots, b_{n-2}\left(V_{n-2}\right) \leq B_{0}, b_{n-1}\left(V_{n-1}\right) \leq B_{0}\right) \leq P\left(B_{0} \leq b \mid b_{1}\left(V_{1}\right) \leq B_{0}, \ldots, b_{n-2}\left(V_{n-2}\right) \leq B_{0}\right) .
$$

Equation 36.

Heuristically, the above equation is justified since $b_{n-1}\left(V_{n-1}\right) \leq B_{0}$ makes it no more likely that $B_{0} \leq b$. It is a well-known fact that can be extended to random variables that are affiliated. ${ }^{6}$ For the sake of self-containment, however, we will prove the above equation in the next lemma.

Lemma 7. Let $k \in \mathbb{N}$ and suppose $X, Y_{1}, \ldots, Y_{k-1}, Y_{k}$ are independent random variables and $d$ is any real number. Then

$$
P\left(X \leq d \mid Y_{1} \leq X\right) \leq P(X \leq d)
$$

Equation 37.

and if $k>1$,

$$
P\left(X \leq d \mid Y_{1} \leq X, \ldots, Y_{k-1} \leq X, Y_{k} \leq X\right) \leq P\left(X \leq d \mid Y_{1} \leq X, \ldots, Y_{k-1} \leq X\right) . \quad \text { Equation } 38 .
$$

Proof. Suppose $X, Y_{1}, \ldots, Y_{k-1}, Y_{k}$ are independent. Note that for any nonnegative real numbers $a, b, c$ such that $b>0$ and $a<b$, we have

$$
\frac{a}{b} \leq \frac{a+c}{b+c}
$$

Equation 39. 
If $k>1$, we have

$$
\begin{aligned}
& P\left(X \leq d \mid Y_{1} \leq X, \ldots, Y_{k-1} \leq X, Y_{k} \leq X\right) \\
&= \frac{P\left(X \leq d, Y_{1} \leq X, \ldots, Y_{k-1} \leq X, Y_{k} \leq X\right)}{P\left(Y_{1} \leq X, \ldots, Y_{k-1} \leq X, Y_{k} \leq X\right)} \\
& \leq \frac{P\left(X \leq d, Y_{1} \leq X, \ldots, Y_{k-1} \leq X, Y_{k} \leq X\right)}{P\left(Y_{1} \leq X, \ldots, Y_{k-1} \leq X, Y_{k} \leq X, Y_{k} \leq d\right)} \\
& \leq \frac{P\left(X \leq d, Y_{1} \leq X, \ldots, Y_{k-1} \leq X, Y_{k} \leq X\right)+P\left(Y_{k} \leq d, Y_{1} \leq X, \ldots, Y_{k-1} \leq X, Y_{k}>X\right)}{P\left(Y_{1} \leq X, \ldots, Y_{k-1} \leq X, Y_{k} \leq X, Y_{k} \leq d\right)+P\left(Y_{k} \leq d, Y_{1} \leq X, \ldots, Y_{k-1} \leq X, Y_{k}>X\right)} \\
&= \frac{P\left(X \leq d, Y_{1} \leq X, \ldots, Y_{k-1} \leq X, Y_{k} \leq d\right)}{P\left(Y_{1} \leq X, \ldots, Y_{k-1} \leq X, Y_{k} \leq d\right)} \\
&= \frac{P\left(X \leq d, Y_{1} \leq X, \ldots, Y_{k-1} \leq X\right) P\left(Y_{k} \leq d\right)}{P\left(Y_{1} \leq X, \ldots, Y_{k-1} \leq X\right) P\left(Y_{k} \leq d\right)} \\
&= \frac{P\left(X \leq d, Y_{1} \leq X, \ldots, Y_{k-1} \leq X\right)}{P\left(Y_{1} \leq X, \ldots, Y_{k-1} \leq X\right)} \\
&= P\left(X \leq d \mid Y_{1} \leq X, \ldots, Y_{k-1} \leq X\right) .
\end{aligned}
$$

Equation 40 .

In the fourth line Equation 39 was applied and the sixth line follows from independence. The proof for the case where $k=1$ is an given by analogous (but simpler) calculation to the one shown above, completing the proof. $^{c}$

With Lemma 7, Equation 36 is justified. Thus, we have

$$
\begin{aligned}
& E\left[\Pi^{S T}\left(v, b_{n}(v)\right) \mid \max _{1 \leq i \leq n-1} b_{i}\left(V_{i}\right) \leq B_{0}\right] \\
& =\left(v-b_{n}(v)\right) P\left(B_{0} \leq b_{n}(v) \mid b_{1}\left(V_{1}\right) \leq B_{0}, \ldots, b_{n-2}\left(V_{n-2}\right) \leq B_{0}, b_{n-1}\left(V_{n-1}\right) \leq B_{0}\right) \\
& \leq\left(v-b_{n}(v)\right) P\left(B_{0} \leq b_{n}(v) \mid b_{1}\left(V_{1}\right) \leq B_{0}, \ldots, b_{n-2}\left(V_{n-2}\right) \leq B_{0}\right) \\
& =E\left[\Pi^{S T}\left(v, b_{n}(v)\right) \max _{1 \leq i \leq n-2} b_{i}\left(V_{i}\right) \leq B_{0}\right] .
\end{aligned}
$$

Equation 41.

Similarly, one can use Lemma 7 to show

$$
E\left[\Pi^{S T}\left(v, b_{n}(v)\right) \mid \max _{1 \leq i \leq 1} b_{i}\left(V_{i}\right) \leq B_{0}\right] \leq E\left[\Pi^{S T}\left(v, b_{n}(v)\right)\right] .
$$

Equation 42.

We can now state a theorem on our expected surplus.

Theorem 8. Let $n>1$. Suppose the conditions of the ST game and of Theorem 5 are satisfied and the functions $b_{n-1}$ and $b_{n}$ are defined as in Theorem 5. Also, define $E\left[\Pi^{S T}(v, b) \mid \max _{1 \leq i \leq 0} b_{i}\left(V_{i}\right) \leq B_{0}\right]$ as $E\left[\Pi^{S T}(v, b)\right]$. If $v=0$ or $v \geq \phi_{n-1}(\omega)$ we have

$$
E\left[\Pi^{S T}\left(v, b_{n-1}(v)\right) \mid \max _{1 \leq i \leq n-2} b_{i}\left(V_{i}\right) \leq B_{0}\right]=E\left[\Pi^{S T}\left(v, b_{n}(v)\right) \mid \max _{1 \leq i \leq n-1} b_{i}\left(V_{i}\right) \leq B_{0}\right] .
$$

Equation 43.

If $0<v<\phi_{n-1}(\omega)$, then

$$
E\left[\Pi^{S T}\left(v, b_{n-1}(v)\right) \mid \max _{1 \leq i \leq n-2} b_{i}\left(V_{i}\right) \leq B_{0}\right]>E\left[\Pi^{S T}\left(v, b_{n}(v)\right) \mid \max _{1 \leq i \leq n-1} b_{i}\left(V_{i}\right) \leq B_{0}\right] .
$$

Equation 44 .

Proof. If $v=0$, then

$$
E\left[\Pi^{S T}\left(0, b_{n-1}(0)\right) \mid \max _{1 \leq i \leq n-2} b_{i}\left(V_{i}\right) \leq B_{0}\right]=0=E\left[\Pi^{S T}\left(0, b_{n}(0)\right) \mid \max _{1 \leq i \leq n-1} b_{i}\left(V_{i}\right) \leq B_{0}\right] .
$$

${ }^{\mathrm{c}}$ The authors would like to thank Byungchul Cha and William Dunham for their contributions to the above proof. In particular, the central idea of the proof of Lemma 7 is due to Dr. Cha. 
If $v \geq \phi_{n-1}(\omega)$, then, as we argued in the proof of Theorem 6, then $v \geq \phi_{n-1}(\omega)>\phi_{n}(\omega)$. Thus, we have

$$
E\left[\Pi^{S T}\left(v, b_{n-1}(v)\right) \mid \max _{1 \leq i \leq n-2} b_{i}\left(V_{i}\right) \leq B_{0}\right]=v-\omega=E\left[\Pi^{S T}\left(v, b_{n}(v)\right) \mid \max _{1 \leq i \leq n-1} b_{i}\left(V_{i}\right) \leq B_{0}\right] . \quad \text { Equation } 46 .
$$

Finally, suppose $0<v<\phi_{n-1}(\omega)$. Using Equation 41 if $n>2$ or Equation 42 if $n=2$, we have

$$
\begin{aligned}
E\left[\Pi^{S T}\left(v, b_{n}(v)\right) \mid \max _{1 \leq i \leq n-1} b_{i}\left(V_{i}\right) \leq B_{0}\right] & \leq E\left[\Pi^{S T}\left(v, b_{n}(v)\right) \mid \max _{1 \leq i \leq n-2} b_{i}\left(V_{i}\right) \leq B_{0}\right] \\
& <E\left[\Pi^{S T}\left(v, b_{n-1}(v)\right) \mid \max _{1 \leq i \leq n-2} b_{i}\left(V_{i}\right) \leq B_{0}\right], \quad \text { Equation } 47 .
\end{aligned}
$$

where in the last line we used the fact that $b_{n-1}(v)$ is the unique maximizer of

$$
E\left[\Pi^{S T}(v, b) \mid \max _{1 \leq i \leq n-2} b_{i}\left(V_{i}\right) \leq B_{0}\right]
$$

Equation 48 .

by either Theorem 5 if $n>2$ or by Theorem 4 if $n=2$, and $b_{n-1}(v) \neq b_{n}(v)$ by Theorem $\mathbf{6}$. This completes the proof.

Heuristically, Theorem 8 says that a bidder can expect more surplus from a ST game in which fewer failed bids have been made. This is despite the fact that a bidder who bids after $n$ failed bids has more information than the $n-1^{s t}$ bidder. The extra information that $b_{n-1}\left(V_{n-1}\right) \leq B_{0}$ is bad news for all bidders, as it signals that $B_{0}$ is potentially high.

\section{SERIAL FIRST-PRICE BUY-OUT AUCTION}

In this section we consider a model which we will call the Serial First-Price Buy-Out (SFPBO) anction. We will give our formal mathematical assumptions below, but we first describe this auction informally. In this auction, bidders place bids in a first-price setting (that is, the highest bidder wins the item and pays her bid). Like the ST game, there is a "buy-out" price $\omega$ (which plays the role of a BIN price) which is the maximum allowed bid for the auction. Unlike the ST game, in the SFPBO auction bidders are directly competing against one another as the seller will accept the highest bid submitted among all bidders. Bidders bid one at a time in an unknown order, and any bidder who submits the buy-out price $\omega$ for a bid will win the auction with certainty and end the auction. Bidders do not know how many previous bids have been made and do not know how many bids will be. Instead, they know a distribution of possible competitors and we will denote the probability of $n$ competing bidders as $q_{n}$ using the notation of Krishna. ${ }^{5}$ Although the bidder who has the chance to bid does not know how many bidders have bid before her, she does know that no previous bidder has submitted the buy-out price (otherwise, the auction would have ended) and this fact will be built into our definition of Nash equilibria for the SFPBO model. We will model the SFPBO auction in one round where each bidder places a bid between 0 and the buy-out price of $\omega$. Below we list our mathematical assumptions for the SFPBO auction.

Definition 9. The conditions of the SFPBO auction are

(i) Fix a number $m \in \mathbb{N}$. We suppose $m+1$ is the maximum amount of possible total bidders in the auction. For each $i \in\{1, \ldots, m+1\}$, the $i^{\text {th }}$ possible bidder's value for the item for auction is represented by the random variable $V_{i}$ with range $[0,1]$. Each $V_{i}$ has a cdf $F(v)$ and a $p d f f(v)$. Note that this means all potential bidder values are identically distributed. Let $\vec{V}=\left(V_{1}, V_{2}, \ldots, V_{m+1}\right)$.

(ii) The pdf satisfies $f(v)>0$ for all $v \in(0,1)$. In particular, the cdf is strictly increasing.

(iii) For all $i \in\{1,2, \ldots, m+1\}$, there is a Bernoulli random variable $X_{i}$ that equals 0 if the $i^{\text {th }}$ possible bidder is not an active bidder and 1 if the $i^{\text {th }}$ possible bidder is an active bidder. We define $B$ to be the index set of active bidders. That is,

$$
B=\left\{j \in\{1, \ldots, m+1\}: X_{j}=1\right\} .
$$

Equation 49. 
Let $\vec{X}=\left(X_{1}, X_{2}, \ldots, X_{m+1}\right)$. We assume that $X_{1}, \ldots, X_{m+1}$ satisfy the following symmetry condition: For any $\vec{\beta} \in$ $\{0,1\}^{m+1}$ and permutation $\sigma$ on $m+1$ elements, we have

$$
P\left[\left(X_{1}, X_{2}, \ldots, X_{m+1}\right)=\left(\beta_{1}, \beta_{2}, \ldots, \beta_{m+1}\right)\right]=P\left[\left(X_{1}, X_{2}, \ldots, X_{m+1}\right)=\left(\beta_{\sigma(1)}, \beta_{\sigma(2)}, \ldots, \beta_{\sigma(m+1)}\right)\right] .
$$

Equation 50.

In particular, note by symmetry, $X_{1}, X_{2}, \ldots, X_{m+1}$ are identically distributed. Also note that we are allowing for the possibility that $X_{1}, X_{2}, \ldots, X_{m+1}$ are correlated.

(iv) Define $p:=P\left(X_{1}=1\right)$. Then $p=P\left(X_{i}=1\right)$ for all $i \in\{1, \ldots, m+1\}$. We also impose the condition $p>0$ so that there is a positive probability that bidders will bid in the auction.

(v) Let $\Sigma$ be a random permutation on $\{1, \ldots, m+1\}$ with a "uniform distribution." That is, for any permutation $\sigma$ on $\{1, \ldots, m+1\}, P(\Sigma=\sigma)=1 /(m+1)$ !. We think of $\Sigma$ as giving the bidders a random order in which to bid in the auction, or equivalent, establishing an order for tie-breaking.

(vi) For any $j \in\{1, \ldots, m+1\}$, the random variables $V_{1}, V_{2}, \ldots V_{m+1}, \Sigma, X_{j}$ are independent.

(vii) Let $N:=X_{1}+X_{2}+\ldots+X_{m+1}=|B|$. That is, $N$ is the number of bidders participating in the auction. For all $n \in\{1,2, \ldots m+1\}$, let $\tilde{q}_{n}=P(N=n)$. That is, $\tilde{q}_{n}$ is the probability distribution of $N$. For all $n \in\{0,1, \ldots m\}$, define $q_{n}$ as $q_{n}=P\left(N=n+1 \mid X_{1}=1\right)$. By the symmetry condition stated in assumption (iii), note that for all $i \in\{1, \ldots, m+1\}$ we have $q_{n}=P\left(N=n+1 \mid X_{i}=1\right)$. That is, for each active bidder $q_{n}$ is the probability that bidder is facing $n$ opponents.

(viii) For each $i \in\{1, \ldots, m+1\}$, define the random variable $Y_{i}=\max _{1 \leq j \leq m+1, i \neq j} X_{j} V_{j}$. Let $G_{i}(y)$ denote the cdf of each $Y_{i}$ dependent on the event $X_{i}=1$. That is,

$$
G_{i}(y):=P\left(Y_{i} \leq y \mid X_{i}=1\right) .
$$

Equation 51.

Also define the random variable $\tilde{Y}=\max _{1 \leq j \leq m+1} X_{j} V_{j}$ and let $\tilde{G}(y)$ denote its $c d f$.

(ix) There exists a maximum bid $\omega$ such that $0<\omega \leq 1$ above which no bids can be placed. That is, all bids must lie in the range $[0, \omega]$.

(x) For any $i \in\{1, \ldots, n\}$ and vector $\vec{y}=\left(y_{1}, y_{2}, \ldots, y_{n}\right) \in \mathbb{R}^{n}$, define $\vec{y}_{-i}=\left(y_{1}, \ldots, y_{i-1}, y_{i+1}, \ldots, y_{n}\right)$ (that is, $\vec{y}_{-i}$ is the vector $\vec{y}$ with the $i^{\text {th }}$ entry omitted). For any function $f: \mathbb{R} \rightarrow \mathbb{R}$ and vector $\vec{y}=\left(y_{1}, y_{2}, \ldots, y_{n}\right) \in \mathbb{R}^{n}$, define $f(\vec{y})=\left(f\left(y_{1}\right), f\left(y_{2}\right), \ldots, f\left(y_{n}\right)\right)$.

(xi) Let $i \in\{1 \ldots, m+1\}$. If $i^{\text {th }}$ possible bidder is actually a bidder and gets the opportunity to bid, then she submits a bid $b_{i}$. The surplus for the $i^{\text {th }}$ possible bidder is

$$
\Pi_{i}\left(V_{i}, b_{i}, \vec{b}_{-i}, \vec{X}_{-i}, \Sigma\right)=\left(V_{i}-b_{i}\right) \mathbb{1}_{b_{i}>\max \left\{b_{j}: j \in B, \Sigma(j)<\Sigma(i)\right\}} \mathbb{1}_{b_{i} \geq \max \left\{b_{j}: j \in B, \Sigma(j)>\Sigma(i)\right\}},
$$

Equation 52.

where we define the maximum over the empty set to be $-\infty$.

(xii) Bidder $i$ will only have the opportunity to bid if $\Sigma(i)=1$ or $\Sigma(i)>1$ and none of bidders $\Sigma^{-1}(1)$ through $\Sigma^{-1}(\Sigma(i)-1)$ bid the maximum bid of $\omega$. Any bidder who bids the maximum bid of $\omega$ automatically wins the auction.

First we elaborate on some of the assumption of the SFPBO auction. In the definition of $\Pi_{i}$ in assumption (xi), we see that the permutation $\Sigma$ determines the tie-breaking order. That is, bidder $\Sigma^{-1}(1)$ wins all ties she is involved with, while $\Sigma^{-1}(2)$ wins all ties except against bidder $\Sigma^{-1}(1)$, etc. We will see that these ties will occur with probability 0 except for when multiple bidders submit the maximum price of $\omega$. That is, tie-breaking will only be important when multiple bidders bid $\omega$. This assumption, along with assumptions (v) and (xii) highlight the fact that bids are submitted one at a time in a serial fashion according to $\Sigma$ and that any bidder who agrees to pay the maximum price of $\omega$ will win the auction and thereby end the auction. In particular, assumption (xii) means that a bidder actually has some extra information about previous 
bidding behavior just from the fact that she has the opportunity to bid. Not only does such a bidder know that she will win the auction with certainty if she submits the maximum bid $\omega$, but she can also infer some information about the previous bidders values if all competing bidders are using a particular bidding function. We will build this fact into our definition of equilibrium strategies below in Definitions 10 and 11.

Before continuing onto the mathematics of the SFPBO auction, we should state here how our model relates to the eBay Best Offer auction. In the eBay best offer auction, potential bidders come across the auction at different times and can decide to end the auction by agreeing to pay the Buy-It-Now price. Our SFPBO auction accounts for the serial nature of this process, as any bidder who has a chance to submit a bid can win the auction instantly by submitting the maximum price $\omega$ as a bid (see assumption (xii)). The serial nature of this model does require an additional layer of complication in the form of the random permutation $\Sigma$ as defined in assumption (v). Also on eBay, bidders do not know how many bidders have submitted bids before them nor how many bidders will submit bids after them. We build this into the SFPBO auction with the unknown number of bidders (see assumptions (iii) and (vii)).

The SFPBO auction differs from the eBay Best Offer auction in two key ways. The first difference is that our model assumes that if no bidder bids the Buy-It-Now price, then the seller will accept the highest offer given. This need not be the case, as the seller has the ability to reject all offers and keep the item. Furthermore, offers on eBay have a time limit of 48 hours. Thus, the best offer the seller receives may have expired by the time the seller decides to accept that offer. The SFPBO auction does not model any of this decision-making required by an actual seller (except for the setting of the maximum bid of $\omega$ ). The second key difference is that in the SFPBO auction (as in the ST game), bidders can submit up to three offers for the same item, and a seller can counteroffer the two first offers made by a bidder. Our model does not account for this bargaining, although the third and final (and uncounterable) offer could be seen as the single bid submitted in the SFPBO auction.

Next we want to classify what sort of bidding functions $b(v)$ we are allowing for the SFPBO auction. As in the work of Reynolds and Wooders, we consider a cut-off strategy. That is, we should have a strategy that is increasing in bidder values up to a "cut-off" point $v_{*}$ after which bidders will bid the buy-out price of $\omega$. Specifically, we have the following definition (which also places some smoothness conditions on our bidding function).

Definition 10. Assume the conditions of the SFPBO auction. Consider a function $b:[0,1] \rightarrow[0, \omega]$ and define $v_{*}(b)$ (or simply $\left.v_{*}\right)$ as

$$
v_{*}(b)=v_{*}:=\inf (\{v: b(v)=\omega\} \cup\{1\}) .
$$

Equation 53.

We say that $b$ is a permissible bidding function for the SFPBO auction (or simply permissible) if

(a) $b$ is nondecreasing on $[0,1]$, and increasing on $\left[0, v_{*}\right]$, and

(b) $b$ is continuous on $\left[0, v_{*}\right]$ and differentiable on $\left(0, v_{*}\right)$.

Note that any permissible bidding function with $v_{*}<1$ is constantly equal to $\omega$ on $\left(v_{*}, 1\right]$. As with most auctions, we cannot hope to find a so-called dominant strategy that maximizes the expected surplus no matter how our opponents bid. The best we can hope for is a type of Nash equilibrium strategy where if all the bidder's opponents use the equilibrium strategy, then the strategy that maximizes the expected profit of the bidder is the same equilibrium strategy. In light of assumption (xii), our equilibrium will actually maximize a bidder's expected value conditioned on the event that bidder had the opportunity to bid.

Definition 11. Assume the conditions of the SFPBO auction. For any $v \in[0,1]$ and $i \in\{1, \ldots, m+1\}$, define the event $C_{v}^{i}$ as

$$
C_{v}^{i}=\left\{X_{j}=0 \text { or } V_{j} \leq v \text { for all } j \in\{1, \ldots, m+1\} \text { such that } \Sigma(j)<\Sigma(i)\right\} .
$$

Equation 54.

That is, $C_{v}^{i}$ is the event that all active bidders who bid before bidder $i$ in the SFPBO anction had a value less than $v$. 
We say that $b:[0,1] \rightarrow[0, \omega]$ is a permissible symmetric Bayesian Nash equilibrium bidding strategy for the SFPBO auction (or just permissible SBNE) if $b$ is permissible and for all $i \in\{1, \ldots, m+1\}$

$$
\sup _{\hat{b} \in[0, \omega]} E\left[\Pi_{i}\left(v_{i}, \hat{b}, b\left(\vec{V}_{-i}\right), \vec{X}_{-i}, \Sigma\right) \mid C_{v_{*}(b)}^{i}, X_{i}=1\right]=E\left[\Pi_{i}\left(v_{i}, b\left(v_{i}\right), b\left(\vec{V}_{-i}\right), \vec{X}_{-i}, \Sigma\right) \mid C_{v_{*}(b)}^{i}, X_{i}=1\right] . \quad \text { Equation } 55 .
$$

Note that our definition of a permissible SBNE includes a conditional expectation to reflect that bidder $i$ knows that all bidders who bid before her who used the bidding function $b$ must have had a value below the cut-off value $v_{*}(b)$ (hence conditioning on the event $C_{v_{*}}^{i}$ ) as well as the fact that she is an active bidder (hence conditioning on the event $X_{i}=1$ ). Given the presence in the definition of a permissible SBNE, we next find the probability of the event $C_{v}^{i}$ given $X_{i}=1$.

Proposition 12. Assume the conditions of the SFPBO auction. Let $v \in[0,1]$ and $i \in\{1, \ldots, m+1\}$. Then

$$
P\left(C_{v}^{i} \mid X_{i}=1\right)=\sum_{n=0}^{m} \frac{1}{n+1}\left(\sum_{k=0}^{n} F(v)^{k}\right) q_{n}=\left\{\begin{aligned}
\sum_{n=0}^{m} \frac{1}{n+1} \frac{1-F(v)^{n+1}}{1-F(v)} q_{n} & \text { if } v<1 \\
1 & \text { if } v=1 .
\end{aligned}\right.
$$

Equation 56.

In particular, $P\left(C_{v}^{i} \mid X_{i}=1\right)$ does not depend on $i$ and is differentiable in $v$.

Proof. If we know that $N=n+1$ for some fixed $n \in\{0, \ldots, m\}$, then we know that random set of bidders $B$ has $n+1$ elements. If $X_{i}=1$, applying the random permutation $\Sigma$ to the elements of $B$ can put the bidder $i$ in any position relative to the other active bidders in $B$ with equal probability (specifically, with probability $\frac{1}{(n+1)}$ ). So, since $\Sigma$ is independent of $\vec{V}$ and each $X_{j}$ we have

$$
\begin{aligned}
P\left(C_{v}^{i} \mid X_{i}=1, N=n+1\right) & =P\left(V_{j} \leq v \text { for all } j \in B \text { such that } \Sigma(j)<\Sigma(i) \mid X_{i}=1, N=n+1\right) \\
& =\sum_{k=0}^{n} F(v)^{k} \frac{1}{n+1}=\frac{1}{n+1} \sum_{k=0}^{n} F(v)^{k} \\
& =\left\{\begin{aligned}
\frac{1}{n+1} \frac{1-F(v)^{n+1}}{1-F(v)} & \text { if } v<1 \\
1 & \text { if } v=1 .
\end{aligned}\right.
\end{aligned}
$$

Equation 57.

Thus, by the rule of total probability we have

$$
\begin{aligned}
P\left(C_{v}^{i} \mid X_{i}=1\right) & =\sum_{n=0}^{m} P\left(C_{v}^{i} \mid X_{i}=1, N=n+1\right) P\left(N=n+1 \mid X_{i}=1\right) \\
& =\sum_{n=0}^{m} P\left(C_{v}^{i} \mid X_{i}=1, N=n+1\right) q_{n} \\
& =\sum_{n=0}^{m} \frac{1}{n+1}\left(\sum_{k=0}^{n} F(v)^{k}\right) q_{n} \\
& = \begin{cases}\sum_{n=0}^{m} \frac{1}{n+1} \frac{1-F(v)^{n+1}}{1-F(v)} q_{n} & \text { if } v<1 \\
1 & \text { if } v=1,\end{cases}
\end{aligned}
$$

Equation 58 .

proving the result.

Next we will derive a unique permissible SBNE for the SFPBO auction by standard auction theory techniques. We will work backwards by first assuming such a permissible SBNE exists and deriving a specific formula for that permissible SBNE. Then will we prove that this formula actually yields a permissible SBNE. First we start with the value of $b$ at 0 .

Proposition 13. Assume the conditions of the SFPBO auction. Let $b:[0,1] \rightarrow[0, \omega]$ be an permissible SBNE for the SFPBO auction. Then $b(0)=0$. 
Proof. First note that a bidder can only bid nonnegative values. That is, $b(0) \geq 0$. In order to show $b(0)=0$, we will proceed by contradiction. Let's assume $b(0) \neq 0$. Thus, we have $b(0)>0$. Since $\left.\Pi_{1}\left(0, b(0), b\left(\vec{V}_{-1}, \vec{X}_{-1}, \Sigma\right)\right)\right)$ will always be negative or zero, we can write

$$
\left.\left.\Pi_{1}\left(0,0, b\left(\vec{V}_{-1}, \vec{X}_{-1}, \Sigma\right)\right)\right)=0 \geq \Pi_{1}\left(0, b(0), b\left(\vec{V}_{-1}, \vec{X}_{-1}, \Sigma\right)\right)\right) .
$$

Equation 59.

Also,

$$
\begin{aligned}
P\left(\Pi_{1}\left(0, b(0), b\left(\vec{V}_{-1}\right), \vec{X}_{-1}, \Sigma\right)<0\right) & =P\left(b(0) \geq \max _{2 \leq i \leq m+1} X_{i} b\left(V_{i}\right)\right) \\
& =P\left(X_{i}=1 \text { and } V_{i}>0 \text { for some } i \in\{2, \ldots, m+1\}\right) \\
& >0 .
\end{aligned}
$$

Equation 60.

Thus $P\left(\Pi_{1}\left(0, b(0), b\left(\vec{V}_{-1}\right), \vec{X}_{-1}, \Sigma\right)<0\right)$ is positive. To put it into words, we have a positive probability of getting a negative surplus when bidding $b(0)$.

So $\Pi_{1}\left(0, b(0), b\left(\vec{V}_{-1}\right), \vec{X}_{-1}, \Sigma\right) \leq 0$ and is strictly less than 0 with positive probability. It follows that,

$$
E\left[\Pi_{1}\left(0, b(0), b\left(\vec{V}_{-0}\right), \vec{X}_{-1}, \Sigma\right) \mid C_{v_{*}}^{1}, X_{1}=1\right]<0=E\left[\Pi_{1}\left(0,0, b\left(\vec{V}_{-0}\right), \vec{X}_{-1}, \Sigma\right) \mid C_{v_{*}}^{1}, X_{1}=1\right] . \quad \text { Equation } 61 .
$$

Therefore, bidding zero will result in a higher expected surplus than bidding $b(0)$. This contradicts our assumption that $b$ is an permissible SBNE. Thus, $b(0) \ngtr 0$. Thus, as a bidder can only bid nonnegative values we have $b(0)=0$ as desired.

Also, we can show that the cut-off value $v_{*}$ must be positive.

Proposition 14. Assume the conditions of the SFPBO auction. Let $b:[0,1] \rightarrow[0, \omega]$ be a permissible SBNE for the SFPBO auction with the associated value $v_{*}$. Then $v_{*}>0$.

Proof. Note that $E\left[\Pi_{1}\left(\omega / 2, \omega, b\left(\vec{V}_{-1}\right), \vec{X}_{-1}, \Sigma\right) \mid C_{v_{*}}^{1}, X_{1}=1\right]=-\omega / 2<0=E\left[\Pi\left(\omega / 2,0, b\left(\vec{V}_{-1}, \vec{X}_{-1}, \Sigma\right) \mid C_{v_{*}}^{1}, X_{1}=1\right]\right.$. Thus, since $b$ is a permissible SBNE, $b(\omega / 2) \neq \omega$. Since $b$ is nondecreasing, this proves that

$$
0<\omega / 2 \leq \inf (\{v: b(v)=\omega\} \cup\{1\})=v_{*},
$$

Equation 62.

as desired.

The next part of our plan is find a formula for a permissible SBNE $b(v)$ over the domain $\left[0, v_{*}\right]$. To do this, we will need to compute the conditional cdf $G_{i}(y)$ of the first order statistic random variable $Y_{i}$. To that end, we have the following definition and proposition.

Definition 15. Assume the conditions of the SFPBO auction. Define the function $G:[0,1] \rightarrow[0,1]$ as

$$
G(y)=\sum_{n=0}^{m} F(y)^{n} q_{n} .
$$

Equation 63.

Proposition 16. Assume the conditions of the SFPBO auction. For each $i \in\{1, \ldots, m+1\}$ the conditional cdf $G_{i}(y)=P\left(Y_{i} \leq\right.$ $\left.y \mid X_{i}=1\right)$ is given by

$$
G_{i}(y)=G(y)=\sum_{n=0}^{m} F(y)^{n} q_{n}
$$

Equation 64.

In particular, $G_{i}$ does not depend on $i$. 
Proof. Let $i \in\{1, \ldots, m+1\}$ be arbitrary. Then we have

$$
\begin{aligned}
G_{i}(y) & =P\left(Y_{i} \leq y \mid X_{i}=1\right) \\
& =\sum_{n=0}^{m} P\left(Y_{i} \leq y \mid N=n+1, X_{i}=1\right) P\left(N=n+1 \mid X_{i}=1\right) \\
& =\sum_{n=0}^{m} F(y)^{n} q_{n},
\end{aligned}
$$

Equation 65.

as desired.

Now we can compute a formula for the bidder's expected surplus.

Proposition 17. Assume the conditions of the SFPBO auction. Let $b:[0,1] \rightarrow[0, \omega]$ be a permissible bidding function for the SFPBO auction with the cut-off value of $v_{*}$, and suppose that $b(0)=0$. Let $b^{-1}$ be the inverse of $\left.b\right|_{\left[0, v_{*}\right]}$ and fix $i \in\{1, \ldots, m+1\}$. If $v \in[0,1]$ and $\hat{b} \in[0, \omega]$, then

$$
E\left[\Pi_{i}\left(v, \hat{b}, b\left(\vec{V}_{-i}\right), \vec{X}_{-i}, \Sigma\right) \mid C_{v_{*}}^{i}, X_{i}=1\right]=\left\{\begin{array}{cll}
\frac{(v-\hat{b}) G\left(b^{-1}(\hat{b})\right)}{P\left(C_{v_{*}}^{i} \mid X_{1}=1\right)} & \text { if } \hat{b} \leq b\left(v_{*}\right) \text { and } \hat{b}<\omega \\
\frac{(v-\hat{b})\left(v_{*}\right)}{P\left(C_{v_{*}} \mid X_{1}=1\right)} & \text { if } b\left(v_{*}\right)<\hat{b}<\omega \\
v-\omega & \text { if } \hat{b}=\omega . & \text { Equation } 66 .
\end{array}\right.
$$

In particular, the above equation holds if $b$ is a permissible $S B N E$ and $E\left[\Pi_{i}\left(v, \hat{b}, b\left(\vec{V}_{-i}\right), \vec{X}_{-i}, \Sigma\right) \mid C_{v_{*}}^{i}, X_{i}=1\right]$ is independent of $i$.

We could rewrite Equation 66 using Proposition 12 but refrain from doing so at the moment for the sake of compactness.

Proof. Note that if $\hat{b}=\omega$, bidder $i$ will win the auction with certainty with surplus $v-\omega$ proving Equation $\mathbf{6 6}$ in the case $\hat{b}=\omega$. To prove the other two cases, suppose $\hat{b}<\omega$. Thus, for any $j$ we have $P\left(b\left(V_{j}\right)=\hat{b}\right)=0$, so we have

$$
\begin{aligned}
E & {\left[\Pi_{i}\left(v, \hat{b}, b\left(\vec{V}_{-i}\right), \vec{X}_{-i}, \Sigma\right) \mid C_{v_{*}}^{i}, X_{i}=1\right] } \\
& =(v-\hat{b}) E\left[\mathbb{1}_{\hat{b}>\max \left\{b\left(V_{j}\right): j \in B, \Sigma(j)<\Sigma(i)\right\}} \mathbb{1}_{\hat{b} \geq \max \left\{b\left(V_{j}\right): j \in B, \Sigma(j)>\Sigma(i)\right\}} \mid C_{v_{*}}^{i}, X_{i}=1\right] \\
& =(v-\hat{b}) P\left(\hat{b} \geq \max \left\{b\left(V_{j}\right): j \in B\right\} \mid C_{v_{*}}^{i}, X_{i}=1\right) \\
& =(v-\hat{b}) P\left(\hat{b} \geq \max \left\{X_{j} b\left(V_{j}\right): 1 \leq j \leq m+1, j \neq i\right\} \mid C_{v_{*}}^{i}, X_{i}=1\right) \\
& =(v-\hat{b}) P\left(\hat{b} \geq \max \left\{b\left(X_{j} V_{j}\right): 1 \leq j \leq m+1, j \neq i\right\} \mid C_{v_{*}}^{i}, X_{i}=1\right),
\end{aligned}
$$

Equation 67.

where on the last line we used the fact that $b(0)=0$. Continuing Equation 67, we have

$$
\begin{aligned}
E & {\left[\Pi_{i}\left(v, \hat{b}, b\left(\vec{V}_{-i}\right), \vec{X}_{-i}, \Sigma\right) \mid C_{v_{*}}^{i}, X_{i}=1\right] } \\
& =(v-\hat{b}) P\left(\hat{b} \geq \max \left\{b\left(X_{j} V_{j}\right): 1 \leq j \leq m+1, j \neq i\right\} \mid C_{v_{*}}^{i}, X_{i}=1\right) \\
& =(v-\hat{b}) P\left(\hat{b} \geq b\left(Y_{i}\right) \mid C_{v_{*}}^{i}, X_{i}=1\right) \\
& =(v-\hat{b}) \frac{P\left(\hat{b} \geq b\left(Y_{i}\right), C_{v_{*}}^{i}, X_{i}=1\right)}{P\left(C_{v_{*}}^{i}, X_{i}=1\right)} .
\end{aligned}
$$

Equation 68. 
Since $\hat{b}<\omega$ by assumption, the event $\hat{b} \geq b\left(Y_{i}\right)$ implies the event $Y_{i} \leq v_{*}$ which in turn implies the event $C_{v_{*}}^{i}$. By this observation, as

$$
\begin{aligned}
& E\left[\Pi_{i}\left(v, \hat{b}, b\left(\vec{V}_{-i}\right), \vec{X}_{-i}, \Sigma\right) \mid C_{v_{*}}^{i}, X_{i}=1\right]=(v-\hat{b}) \frac{P\left(\hat{b} \geq b\left(Y_{i}\right), C_{v_{*}}^{i}, X_{i}=1\right)}{P\left(C_{v_{*}}^{i}, X_{i}=1\right)} \\
& =(v-\hat{b}) \frac{P\left(\hat{b} \geq b\left(Y_{i}\right), X_{i}=1\right)}{P\left(C_{v_{*}}^{i}, X_{i}=1\right)} \\
& =(v-\hat{b}) \frac{P\left(\hat{b} \geq b\left(Y_{i}\right) \mid X_{i}=1\right)}{P\left(C_{v_{*}}^{i} \mid X_{i}=1\right)} \\
& =\left\{\begin{array}{cl}
(v-\hat{b}) \frac{P\left(b^{-1}(\hat{b}) \geq Y_{i} \mid X_{i}=1\right)}{P\left(C_{v_{*}}^{i} \mid X_{i}=1\right)} & \text { if } \hat{b} \leq b\left(v_{*}\right) \text { and } \hat{b}<\omega \\
(v-\hat{b}) \frac{P\left(v_{*} \geq Y_{i} \mid X_{i}=1\right)}{P\left(C_{v_{*}}^{i} \mid X_{i}=1\right)} & \text { if } b\left(v_{*}\right)<\hat{b}<\omega
\end{array}\right. \\
& =\left\{\begin{array}{cl}
\frac{(v-\hat{b}) G\left(b^{-1}(\hat{b})\right)}{P\left(C_{v_{*}}^{i} \mid X_{1}=1\right)} & \text { if } \hat{b} \leq b\left(v_{*}\right) \text { and } \hat{b}<\omega . \\
\frac{(v-\hat{b}) G\left(v_{*}\right)}{P\left(C_{v_{*}}^{1} \mid X_{1}=1\right)} & \text { if } b\left(v_{*}\right)<\hat{b}<\omega
\end{array},\right.
\end{aligned}
$$

Equation 69.

where we used Proposition 12 and Proposition 16 in the last line. This completes the proof.

The above proposition will allow us to derive a formula for the equilibrium bidding function $b(v)$ over $\left[0, v_{*}\right]$. However, before we derive such a formula, we note $(v-\hat{b}) \frac{P\left(\hat{b} \geq b\left(Y_{i}\right), C_{v_{*}}^{i}, X_{i}=1\right)}{P\left(C_{v_{*}}^{i}, X_{i}=1\right)}=(v-\hat{b}) \frac{P\left(\hat{b} \geq b\left(Y_{i}\right), X_{i}=1\right)}{P\left(C_{v_{*}}^{i}, X_{i}=1\right)}$ above. This equation tells us that for any bid $\hat{b}<\omega$, the fact the event $C_{v_{*}}^{i}$ has occurred does not affect the probability of the event of having the highest bid among all opponents (i.e. the event $\hat{b} \geq b\left(Y_{i}\right)$ ). That is, a bidder who bid an amount $\hat{b}$ that is less than the buy-out price $\omega$ does not need to take into account that any previous bidders must have a value below the cut-off of $v_{*}$, because for her to win the auction all bidders must have values below the cut-off value. This fact will mean that the conditional nature of the expected value on $C_{v_{*}}^{i}$ will not profoundly affect the equilibrium behavior for bidders whose values fall below the cut-off $v_{*}$. Thus, one could expect that the equilibrium found in an auction with simultaneous bidding and a random tie-breaker for bidders who bid $\omega$ is the same as one found in a SFPBO auction. We will show that this is indeed the case in the next section. But now we return to deriving the formula for the equilibrium bidding function over $\left[0, v_{*}\right]$ for the SFPBO auction.

Theorem 18. Assume the conditions of the SFPBO auction. Let $b:[0,1] \rightarrow[0, \omega]$ be an permissible SBNE for the SFPBO auction. Then, if $v \in\left[0, v_{*}\right]$

$$
b(v)=\left\{\begin{aligned}
v-\frac{\int_{0}^{v} G(y) d y}{G(v)} & \text { if } 0<v \leq v_{*} \\
0 & \text { if } v=0 .
\end{aligned}\right.
$$

Equation 70.

The derivation of Equation 70 is entirely analogous to the derivation of the SBNE of a first-price sealed-bid auction and that derivation is well-known. ${ }^{2,5,6}$ We still give a proof of Equation 70 for the sake of self-containment.

Proof. Let $x, y \in\left(0, v_{*}\right)$. By Proposition 17,

$$
E\left[\Pi_{i}\left(y, b(x), b\left(\vec{V}_{-1}\right), \vec{X}_{-1}, \Sigma\right) \mid C_{v_{*}}^{1}, X_{1}=1\right]=\frac{(y-b(x)) G(x)}{P\left(C_{v_{*}}^{1} \mid X_{1}=1\right)} .
$$

Then, taking the derivative of our expected surplus with respect to $x$, we have:

$$
\frac{d}{d x} E\left[\Pi\left(y, b(x), b\left(\vec{V}_{-0}\right)\right)\right]=\frac{1}{P\left(C_{v_{*}}^{1} \mid X_{1}=1\right)}\left((y-b(x)) G^{\prime}(x)-b^{\prime}(x) G(x)\right) .
$$

Equation 72. 
Since $b$ is an permissible SBNE, we know $E\left[\Pi_{i}\left(y, b(x), b\left(\vec{V}_{-1}\right), \vec{X}_{-1}, \Sigma\right) \mid C_{v_{*}}^{1}, X_{1}=1\right]$ is maximized when $x=y$. Thus we must have a critical point at $x=y$. So,

$$
0=y G^{\prime}(y)-b(y) G^{\prime}(y)-b^{\prime}(y) G(y) .
$$

Equation 73.

Adding $b(y) G^{\prime}(y)+b^{\prime}(y) G(y)$ to both sides to the above equation yields

$$
y G^{\prime}(y)=G(y) b^{\prime}(y)+G^{\prime}(y) b(y)=\frac{d}{d y}[G(y) b(y)] \text { for all } y \in\left(0, v_{*}\right) .
$$

Then, for any $v \in\left(0, v_{*}\right]$, we can integrate both sides of the above from 0 to $v$ and have:

$$
G(v) b(v)-G(0) b(0)=\int_{0}^{v} \frac{d}{d y}(G(y) b(y)) d y=\int_{0}^{v} y G^{\prime}(y) d y .
$$

Equation 75.

Note that $b(0)=0$ by Proposition 13, and so we can rewrite Equation 75 as:

$$
G(v) b(v)=\int_{0}^{v} y G^{\prime}(y) d y
$$

Equation 76.

Integrating by parts, we have

$$
G(v) b(v)=\left.y G(y)\right|_{0} ^{v}-\int_{0}^{v} G(y) d y=v G(v)-\int_{0}^{v} G(y) d y
$$

Equation 77.

Let $v>0$. Then $G(v)>0$ and we can divide both sides of our function by $G(v)$ to get $b(v)=v-\frac{\int_{0}^{v} G(y) d y}{G(v)}$. Since $b(0)=0$ by Proposition 13, this completes the proof.

Theorem 18 gives us a necessary formula for our permissible SBNE over $\left[0, v_{*}\right]$. This formula would look familiar to anyone who has studied auction theory, as it is the standard SBNE formula for a first-price sealed-bid auction with our particular conditional cdf $G(y)$ for the first order statistic $Y_{i}$. Our SFPBO auction is different as the maximum bid is capped by $\omega$, and thus we have to reckon with how we can find $v_{*}$, the value above which bidders will just use the buy-out price $\omega$. To that end, we define a function inspired by Theorem 18. Define $\tilde{b}:[0,1] \rightarrow[0,1]$ as

$$
\tilde{b}(v):=\left\{\begin{aligned}
v-\frac{\int_{0}^{v} G(y) d y}{G(y)} & \text { if } 0<v \leq 1 . \\
0 & \text { if } v=0 .
\end{aligned}\right.
$$

$\tilde{b}$ would be the SBNE for a first-price sealed-bid auction with no reserve, an unknown number of bidders, and no buy-out price. ${ }^{5}$ The content of Theorem 18 can be restated as: if $b$ is a permissible SBNE for the SFPBO auction, then for all $v \in\left[0, v_{*}\right]$ we have $b(v)=\tilde{b}(v)$. To characterize $v_{*}$, we define a new function $\Delta$ that is meant to measure the difference in expected surplus to a bidder by bidding $\tilde{b}(v)$ or bidding $\omega$.

Definition 19. Assume the conditions of the SFPBO auction. Define the function $\Delta:[0,1] \rightarrow \mathbb{R}$ by

$$
\Delta(v):=(v-\tilde{b}(v)) \frac{G(v)}{P\left(C_{v}^{1} \mid X_{1}=1\right)}-(v-\omega)=\frac{\int_{0}^{v} G(y) d y}{P\left(C_{v}^{1} \mid X_{1}=1\right)}-v+\omega
$$

Equation 79.

Note that by Proposition 12, we have

$$
\Delta(v)=\left\{\begin{array}{rr}
\frac{(1-F(v)) \int_{0}^{v} G(y) d y}{\sum_{n=0}^{m} \frac{1}{n+1}\left(1-F(v)^{n+1}\right) q_{n}}-v+\omega & \text { if } v<1 \\
\int_{0}^{1} G(y) d y-1+\omega & \text { if } v=1 .
\end{array}\right.
$$


Again, $\Delta$ is meant to measure which of the bidding options (making an offer or using the buy-out price) result in the most expected surplus for the bidder. If $\Delta(v)>0$, then the bidder should choose the first option and if $\Delta(v)<0$, then she should choose the second. If $\Delta(v)=0$, she should be indifferent to either option. Thus, we expect that $\Delta\left(v_{*}\right)=0$ if $v_{*}<1$, as this is the value where the bidder switches from the first option to the second. Below, we will prove these heuristic observations.

Proposition 20. Assume the conditions of the SFPBO auction and define $\Delta$ as in Definition 19. Then $\Delta$ is continuous on $[0,1]$, differentiable on $(0,1), \Delta(0)>0$ and $\Delta$ is decreasing on $[0,1]$.

To prove the above, we will need an auxillary definition and a lemma:

Definition 21. Let $n$ be a natural number. Define the polynomial $j_{n}(x)$ as

$$
j_{n}(x):=-(n-1) x^{n}+n x^{n-1}-1 .
$$

Equation 81.

Lemma 22. For any natural number $n, j_{n}(x)=-(n-1) x^{n}+n x^{n-1}-1$ is negative for all $x \in[0,1)$.

Proof. First note that $j_{n}(0)=-1<0$ and $j_{n}(1)=0$. Thus, to prove the lemma it suffices to show that $j_{n}^{\prime}(x)>0$ for $x \in(0,1)$. To that end, note that $j_{n}^{\prime}(x)=n(n-1) x^{n-2}(1-x)>0$ for $x \in(0,1)$, completing the proof.

Now we are ready to prove our proposition involving $\Delta$ :

Proof. (Proposition 20) First note that we can rewrite Equation 80 as

$$
\Delta(v)=\frac{\int_{0}^{v} G(y) d y}{\sum_{n=0}^{m} \frac{1}{n+1}\left(\sum_{k=0}^{n} F(v)^{k}\right) q_{n}}-v+\omega,
$$

Equation 82.

and thus $\Delta$ is continuous on $[0,1]$ and differentiable on $(0,1)$ by the Fundamental Theorem of Calculus. Next note $\Delta(0)=$ $\omega>0$. To prove $\Delta$ is decreasing, we simply prove its derivative is negative on $(0,1)$. Thus, for $v \in(0,1)$, we have

$$
\begin{aligned}
\Delta^{\prime}(v)= & \frac{d}{d v}\left(\frac{(1-F(v)) \int_{0}^{v} G(y) d y}{\sum_{n=0}^{m} \frac{1}{n+1}\left(1-F(v)^{n+1}\right) q_{n}}-v+\omega\right) \\
= & \frac{\sum_{n=0}^{m}\left(\frac{1}{n+1}\left(1-F(v)^{n+1}\right) q_{n}\right)\left(-f(v) \int_{0}^{v} G(y) d y+(1-F(v)) G(v)\right)+(1-F(v)) F(v)^{n} f(v) q_{n} \int_{0}^{v} G(y) d y}{\left(\sum_{n=0}^{m} \frac{1}{n+1}\left(1-F(v)^{n+1}\right) q_{n}\right)^{2}}-1 \\
= & \frac{-f(v) \int_{0}^{v} G(y) d y \sum_{n=0}^{m} \frac{q_{n}}{n+1}\left(1-F(v)^{n+1}-(n+1)(1-F(v)) F(v)^{n}\right)}{\left(\sum_{n=0}^{m} \frac{1}{n+1}\left(1-F(v)^{n+1}\right) q_{n}\right)^{2}}+ \\
& \frac{\sum_{n=0}^{m} \frac{q_{n}}{n+1}\left((n+1)(1-F(v)) F(y)^{n}-\left(1-F(v)^{n+1}\right)\right)}{\sum_{n=0}^{m} \frac{1}{n+1}\left(1-F(v)^{n+1}\right) q_{n}} \\
& =\frac{-f(v) \int_{0}^{v} G(y) d y \sum_{n=0}^{m} \frac{q_{n}}{n+1}\left(-j_{n}(F(v))\right)}{\left(\sum_{n=0}^{m} \frac{1}{n+1}\left(1-F(v)^{n+1}\right) q_{n}\right)^{2}}-\frac{\sum_{n=0}^{m} \frac{q_{n}}{n+1}\left(-j_{n}(F(v))\right)}{\sum_{n=0}^{m} \frac{1}{n+1}\left(1-F(v)^{n+1}\right) q_{n}} \\
< & 0 \quad \text { Equation 83. }
\end{aligned}
$$

for $v \in(0,1)$ (applying Lemma 22 in the last inequality). This completes the proof.

Next we will show that $\Delta(v)$ is non-negative for $v \leq v_{*}$ : 
Proposition 23. Assume the conditions of the SFPBO auction, suppose that there exists a permissible SBNE $b$ for this auction, and define $\Delta$ as in Definition 19. Then $\Delta\left(v_{*}\right) \geq 0$ and $\Delta(v)>0$ for all $v \in\left[0, v_{*}\right)$.

Proof. Let $v \in\left[0, v_{*}\right]$. Using Proposition 17 and Theorem 18 we have

$$
\begin{aligned}
\Delta(v) & =(v-\tilde{b}(v)) \frac{G(v)}{P\left(C_{v}^{1} \mid X_{1}=1\right)}-(v-\omega) \\
& =(v-b(v)) \frac{G(v)}{\sum_{n=0}^{m} \frac{1}{n+1}\left(\sum_{k=0}^{n} F(v)^{k}\right) q_{n}}-(v-\omega) \\
& >(v-b(v)) \frac{G(v)}{\sum_{n=0}^{m} \frac{1}{n+1}\left(\sum_{k=0}^{n} F\left(v_{*}\right)^{k}\right) q_{n}}-(v-\omega) \\
& =E\left[\Pi_{1}\left(v, b(v), b\left(\vec{V}_{-1}\right), \vec{X}_{-1}, \Sigma\right) \mid C_{v_{*}}^{1}, X_{1}=1\right]-E\left[\Pi_{1}\left(v, \omega, b\left(\vec{V}_{-1}\right), \vec{X}_{-1}, \Sigma\right) \mid C_{v_{*}}^{1}, X_{1}=1\right] \\
& \geq 0,
\end{aligned}
$$

Equation 84.

where the last inequality is justified because $b$ is a permissible $\operatorname{SBNE}$. Thus, $\Delta(v) \geq 0$ for $v \leq v_{*}$. Since $\Delta$ is decreasing, for $v<v_{*}$ we have $\Delta(v)>\Delta\left(v_{*}\right) \geq 0$, completing the proof.

Heuristically, we assume that any equilibrium strategy for the SFPBO auction should have a jump discontinuity at $v_{*}$ in the case that $v_{*}<1$, as bidders should be willing to bid a higher amount to account for the certainty in using the buy-out price $\omega$. Using Proposition 23 we can prove this as a fact. Before we do so, we prove a lemma that will be useful in that and subsequent proofs.

Lemma 24. Define $\Psi:[0,1] \rightarrow[0,1]$ as $\Psi(v)=G(v)-P\left(C_{v}^{1} \mid X_{1}=1\right)$. Then $\Psi(v)<0$ for $v \in[0,1)$ and $\Psi(1)=0$.

Proof. Let $v \in[0,1]$. We compute

$$
\begin{aligned}
\Psi(v) & =G(v)-P\left(C_{v}^{1} \mid X_{1}=1\right) \\
& =\sum_{n=0}^{m} \frac{1}{(n+1)(1-F(v))}\left((n+1) F(v)^{n}(1-F(v))-\left(1-F(v)^{n+1}\right)\right) q_{n} \\
& =\sum_{n=0}^{m} \frac{1}{(n+1)(1-F(v))} j_{n+1}(F(v)) q_{n} .
\end{aligned}
$$

Equation 85.

Equation 86.

The result then follows from the facts that $0 \leq F(v) \leq 1, F(v)=1$ if and only if $v=1$, and Lemma 22 .

Now we can prove our jump discontinuity proposition:

Proposition 25. Assume the conditions of the SFPBO auction, suppose that there exists a permissible SBNE b for this auction with corresponding value $v_{*}$, and define $\Delta$ as in Definition 19. If $v_{*}<1$, then $b\left(v_{*}\right)<\omega$.

Proof. Suppose $v_{*}<1$ and $b\left(v_{*}\right)=\omega$ in order to derive a contradiction. Since $b\left(v_{*}\right)=\tilde{b}\left(v_{*}\right)$ and we assume $b\left(v_{*}\right)=\omega$ we have

$$
\begin{aligned}
\Delta\left(v_{*}\right) & =\left(v_{*}-b\left(v_{*}\right)\right) \frac{G\left(v_{*}\right)}{P\left(C_{v_{*}}^{1} \mid X_{1}=1\right)}-\left(v_{*}-\omega\right) \\
& =\left(v_{*}-\omega\right)\left(\frac{G\left(v_{*}\right)}{P\left(C_{v_{*}}^{1} \mid X_{1}=1\right)}-1\right)<0,
\end{aligned}
$$

Equation 87.

where in the last inequality we used the fact that $v_{*}<1$ and Lemma 24. However, by Proposition 23, $\Delta\left(v_{*}\right) \geq 0$, which is a contradiction. Thus, if $v_{*}<1$, then $b\left(v_{*}\right)<\omega$. 
If $v_{*}<1$, the value $v_{*}$ of a permissible SBNE $b$ is a tipping point value where a risk-neutral bidder should be indifferent between submitting an offer or just purchasing the item for $\omega$. Since $\Delta$ measures the difference in expected surplus between those two options, we thus expect that $\Delta\left(v_{*}\right)=0$. If $v_{*}=1$, then $\omega$ is too high for a bidder to gain more expected surplus by submitting the buy-out price of $\omega$ than just submitting an offer. We can now prove these two observations.

Proposition 26. Assume the conditions of the SFPBO auction, suppose that there exists a permissible SBNE $b:[0,1] \rightarrow[0, \omega]$ for this auction with corresponding value $v_{*}$, and define $\Delta$ as in Definition 19. If $v_{*}=1$, then $\Delta(v)>0$ on $[0,1)$ and $\Delta\left(v_{*}\right) \geq 0$. If $v_{*}<1$, then $\Delta\left(v_{*}\right)=0$.

Proof. If $v_{*}=1$, the result directly follows from Proposition 23. So suppose $v_{*}<1$. By Proposition 25, $b\left(v_{*}\right)<\omega$. Let $v \in\left(v_{*}, 1\right]$ be arbitrary. Since $b$ is a permissible SBNE, we have by Proposition 17

$$
\begin{aligned}
0 & \geq E\left[\Pi\left(v, b\left(v_{*}\right), b\left(\vec{V}_{-1}\right), X_{-1}, \Sigma\right) \mid C_{v_{*}}^{1}, X_{1}=1\right]-E\left[\Pi\left(v, b(v), b\left(\vec{V}_{-1}\right), X_{-1}, \Sigma\right) \mid C_{v_{*}}^{1}, X_{1}=1\right] \\
& =\left(v-\tilde{b}\left(v_{*}\right)\right) \frac{G\left(v_{*}\right)}{P\left(C_{v_{*}}^{1} \mid X_{1}=1\right)}-(v-\omega) \\
& =(v-\tilde{b}(v)) \frac{G(v)}{P\left(C_{v}^{1} \mid X_{1}=1\right)}-(v-\omega)+\left(v-\tilde{b}\left(v_{*}\right)\right) \frac{G\left(v_{*}\right)}{P\left(C_{v_{*}}^{1} \mid X_{1}=1\right)}-(v-\tilde{b}(v)) \frac{G(v)}{P\left(C_{v}^{1} \mid X_{1}=1\right)} \\
& =\Delta(v)+\left(v-\tilde{b}\left(v_{*}\right)\right) \frac{G\left(v_{*}\right)}{P\left(C_{v_{*}}^{1} \mid X_{1}=1\right)}-(v-\tilde{b}(v)) \frac{G(v)}{P\left(C_{v}^{1} \mid X_{1}=1\right)} \\
& =\Delta(v)+\frac{\left(v-v_{*}\right) G\left(v_{*}\right)+\int_{0}^{v_{*}} G(y) d y}{P\left(C_{v_{*}}^{1} \mid X_{1}=1\right)}-\frac{\int_{0}^{v} G(y) d y}{P\left(C_{v}^{1} \mid X_{1}=1\right)} .
\end{aligned}
$$

Equation 88 .

Since $\Delta$ is continuous at $v_{*}$ and $v_{*}<1$, we have

$$
\begin{aligned}
\Delta\left(v_{*}\right) & =\lim _{v \rightarrow v_{*}^{+}} \Delta(v) \\
& \leq \lim _{v \rightarrow v_{*}^{+}} \frac{\int_{0}^{v} G(y) d y}{P\left(C_{v}^{1} \mid X_{1}=1\right)}-\frac{\left(v-v_{*}\right) G\left(v_{*}\right)+\int_{0}^{v_{*}} G(y) d y}{P\left(C_{v_{*}}^{1} \mid X_{1}=1\right)} \\
& =\frac{\int_{0}^{v_{*}} G(y) d y}{P\left(C_{v_{*}}^{1} \mid X_{1}=1\right)}-\frac{\left(v_{*}-v_{*}\right) G\left(v_{*}\right)+\int_{0}^{v_{*}} G(y) d y}{P\left(C_{v_{*}}^{1} \mid X_{1}=1\right)} \\
& =0 .
\end{aligned}
$$

Equation 89.

Thus, $\Delta\left(v_{*}\right) \leq 0$. But by Proposition 23, $\Delta\left(v_{*}\right) \geq 0$. Thus $\Delta\left(v_{*}\right)=0$, as desired.

We now have completely characterized the permissible SBNE in a SFPBO auction which we summarize in the following theorem.

Theorem 27. Assume the conditions of the SFPBO auction, suppose that there exists a permissible SBNE $b:[0,1] \rightarrow[0, \omega]$ for this auction with corresponding value $v_{*}$, and define $\Delta$ as in Definition 19. If $\Delta(v) \geq 0$ for all $v \in[0,1]$, then

$$
b(v)=\left\{\begin{array}{rl}
0 & \text { for } v=0 \\
v-\frac{\int_{0}^{v} G(y) d y}{G(v)} & \text { for } 0<v
\end{array} .\right.
$$

Equation 90.

If $\Delta(v)<0$ for some $v \in[0,1]$, then

$$
b(v)=\left\{\begin{aligned}
0 & \text { for } v=0 \\
v-\frac{\int_{0}^{v} G(y) d y}{G(y)} & \text { for } 0<v \leq v_{*} . \\
\omega & \text { for } v>v_{*}
\end{aligned}\right.
$$


Moreover, $b(v)$ has a jump discontinuity at $v_{*}$ and $v_{*}$ is the unique value in $[0,1]$ such that $\Delta\left(v_{*}\right)=0$.

Proof. First suppose $\Delta(v) \geq 0$ for all $v \in[0,1]$. If $v_{*}<1$, then by Proposition $\mathbf{2 6} \Delta\left(v_{*}\right)=0$ and since $\Delta$ is decreasing, we have $\Delta(1)<0$, which contradicts our assumption. Thus, $v_{*}=1$ and Equation 90 follows from Theorem 18. Next suppose $\Delta(v)<0$ for some $v \in[0,1]$. If $v_{*}=1$, then by Proposition $23 \Delta(v) \geq 0$ for all $v \in[0,1]$, a contradiction. Thus, $v_{*}<1$ and Equation 91 and the comment following it follow from Theorem 18, Proposition 25, and Proposition 26, completing the proof.

Note that if Equation 90 holds, the buy-out price $\omega$ is higher than any bidder is willing to offer in equilibrium. However, if Equation 91 holds, not only are some bidders with values $v_{*}<v$ willing to bid $\omega$, but since there is a jump discontinuity some of them will bid $\omega$ even though the bid $\tilde{b}(v)$ is lower than the buy-out price. That is, the buy-out price actually can induce higher bids in equilibrium than the standard first-price sealed-bid auction that does not have a buy-out. Despite this jump discontinuity, we will show in our last section that a seller maximizes revenue by setting a buy-out price that is higher than any bidder is willing to pay in equilibrium.

While Theorem 27 gives us the exact necessary conditions for a permissible SBNE in the SFPBO auction, we still need to prove this formula gives an actual permissible SBNE. That is, we have proven a uniqueness statement in the previous theorem, but we still need to prove existence. Proving that the formulas Equation $\mathbf{9 0}$ and Equation 91 actually give permissible SBNEs the content of the proof of the next theorem.

Theorem 28. Assume the conditions of the SFPBO auction, define $\Delta$ as in Definition 19, and define $v_{*}$ as

$$
v_{*}=\inf (\{v \in[0,1]: \Delta(v)<0\} \cup\{1\})
$$

Equation 92.

Then the function $b:[0,1] \rightarrow[0,1]$ given by

$$
b(v)=\left\{\begin{array}{rl}
0 & \text { for } v=0 \\
v-\frac{\int_{0}^{v} G(y) d y}{G(v)} & \text { for } 0<v \leq v_{*} . \\
\omega & \text { for } v>v_{*}
\end{array} .\right.
$$

Equation 93.

is the unique permissible SBNE for the SFPBO auction.

Proof. The uniqueness part of this statement was proven in Theorem 27, thus it only remains to prove that $b$ as defined in Equation 93 yields a permissible SBNE. Due to the symmetry of $\vec{V}$ and $\vec{X}$ as well as the fact that the quantities $P\left(C_{v_{*}}^{i} \mid X_{i}=\right.$ 1) (Proposition 12) and $E_{i}\left[v, \hat{b}, b\left(\vec{V}_{-i}\right), \vec{X}_{-i}, \Sigma \mid C_{v_{*}}^{i}, X_{i}=1\right]$ (Proposition 17) are independent of $i$, it suffices to prove the result for $i=1$. First we record some facts about $\Delta$ and $v_{*}$. Note that since $\Delta(0)=\omega>0$ and $\Delta$ is continuous, we must have $v_{*}>0$. Also by continuity and the fact that $\Delta$ is decreasing, $\Delta\left(v_{*}\right) \geq 0$ and thus $\Delta(v)>0$ for $v<v_{*}$. Finally, if $v_{*}<1$, again by continuity $\Delta\left(v_{*}\right)=0$ and since $\Delta$ is decreasing if $v>v_{*}$, then $\Delta(v)<0$.

Next we will show $b$ is permissible. Note that $b$ is differentiable on $\left(0, v_{*}\right)$ and continuous on $\left(0, v_{*}\right]$ by the Fundamental Theorem of Calculus. To show that $b$ is also continuous at 0 we can employ a Squeeze Theorem argument. To that end, first note that $G^{\prime}(y)>0$ since $F^{\prime}(y)=f(y)>0$ for all $y \in(0,1)$, so we have

$$
b(v)=\int_{0}^{v} y G^{\prime}(y) d y>0 \text { for } v \in\left(0, v_{*}\right) .
$$

Equation 94.

Also, for $v \in\left(0, v_{*}\right), b(v)<v$. Thus, by the Squeeze Theorem, $\lim _{v \rightarrow 0^{+}} b(v)=0=b(0)$, proving continuity at 0 . To show that $b$ is increasing on $\left[0, v_{*}\right]$, we will show $b^{\prime}(v)>0$ for $v \in\left(0, v_{*}\right)$. Again noting that $G^{\prime}(v)>0$ for $v \in\left(0, v_{*}\right)$, we have

$$
b^{\prime}(v)=1-\frac{G(v)^{2}-G^{\prime}(v) \int_{0}^{v} G(y) d y}{G(v)^{2}}=\frac{G^{\prime}(v) \int_{0}^{v} G(y) d y}{G(v)^{2}}>0 .
$$

Equation 95. 
To prove that $b$ is nondecreasing on $[0,1]$ and $v_{*}=\inf (\{v \in[0,1]: b(v)=\omega\} \cup\{1\})$, it suffices to show that $b(v)<\omega$ for $v<v_{*}$. Suppose $b(v)=\omega$ for some $v<v_{*}$ to derive a contradiction. Since $v<v_{*} \leq 1$, we have $G(v)-P\left(C_{v}^{1} \mid X_{1}=1\right)<0$ by Lemma 24. So we have

$$
0<\Delta(v)=(v-b(v)) \frac{G(v)}{P\left(C_{v}^{1} \mid X_{1}=1\right)}-(v-\omega)=(v-\omega)\left(\frac{G(v)}{P\left(C_{v}^{1} \mid X_{1}=1\right)}-1\right)<0,
$$

Equation 96.

giving us our desired contradiction. Thus, $b(v)<\omega$ for all $v<v_{*}$ and we have shown that $b$ is permissible.

Next we show that $b$ is a permissible SBNE. Note that for any $v \in[0,1]$, by Proposition 17 , if we have $\hat{b} \in\left(b\left(v_{*}\right), \omega\right)$, then

$$
\begin{aligned}
E\left[\Pi_{1}\left(v, \hat{b}, b\left(\vec{V}_{-1}\right), X_{-1}, \Sigma\right) \mid C_{v_{*}}^{1}, X_{1}=1\right] & =\frac{(v-\hat{b}) G\left(v_{*}\right)}{P\left(C_{v_{*}}^{1} \mid X_{1}=1\right)} \\
& <\frac{\left(v-b\left(v_{*}\right)\right) G\left(v_{*}\right)}{P\left(C_{v_{*}}^{1} \mid X_{1}=1\right)} \\
& =E\left[\Pi_{1}\left(v, b\left(v_{*}\right), b\left(\vec{V}_{-1}\right), X_{-1}, \Sigma\right) \mid C_{v_{*}}^{1}, X_{1}=1\right] . \text { Equation } 97 .
\end{aligned}
$$

Since $b(0)=0$, to prove $b$ is a permissible SBNE it suffices to show that for all $v \in[0,1]$,

$$
E\left[\Pi_{1}\left(v, b(v), b\left(\vec{V}_{-1}\right), X_{-1}, \Sigma\right) \mid C_{v_{*}}^{1}, X_{1}=1\right]=\sup _{\hat{b} \in\left[b(0), b\left(v_{*}\right)\right] \cup\{\omega\}} E\left[\Pi_{1}\left(v, \hat{b}, b\left(\vec{V}_{-1}\right), X_{-1}, \Sigma\right) \mid C_{v_{*}}^{1}, X_{1}=1\right]
$$

To that end, for any $x \in\left[0, v_{*}\right) \cup\left\{v \mid v=v_{*}\right.$ and $\left.b(v)<\omega\right\}$, we have by Proposition 17

Equation 98.

$$
\begin{aligned}
E\left[\Pi_{1}\left(v, b(x), b\left(\vec{V}_{-1}\right), X_{-1}, \Sigma\right) \mid C_{v_{*}}^{1}, X_{1}=1\right] & =\frac{(v-b(x)) G(x)}{P\left(C_{v_{*}}^{1}, X_{1}=1\right)} \\
& =\frac{1}{P\left(C_{v_{*}}^{1}, X_{1}=1\right)}\left((v-x) G(x)+\int_{0}^{x} G(y) d y\right) \text { Equation } 99 .
\end{aligned}
$$

Taking the derivative of the above with respect to $x$ (for $x \in\left(0, v_{*}\right)$ ) yields

$$
\frac{d}{d x} E\left[\Pi_{1}\left(v, b(x), b\left(\vec{V}_{-1}\right), X_{-1}, \Sigma\right) \mid C_{v_{*}}^{1}, X_{1}=1\right]=\frac{1}{P\left(C_{v_{*}}^{1}, X_{1}=1\right)}(v-x) G^{\prime}(x) .
$$

Equation 100.

Since $G^{\prime}(x)=\sum_{n=1}^{m} n F(x)^{n-1} f(x) q_{n}>0$ for all $x$ in $(0,1)$, the above derivative is positive for $x<v$ and negative for $x>v$. Thus, in the case where $v<v_{*}$, we have a global maximum for our conditional expected value at $b(v)$ over the set $\left[b(0), b\left(v_{*}\right)\right) \cup\left\{b(v) \mid v=v_{*}\right.$ and $\left.b(v)<\omega\right\}$. In the case where $v \geq v_{*}$, then we have

$$
\left.\sup _{\hat{b} \in\left[b(0), b\left(v_{*}\right)\right) \cup\left\{b(v) \mid v=v_{*}, b(v)<\omega\right\}} E\left[\Pi_{1}\left(v, \hat{b}, b\left(\vec{V}_{-1}\right), X_{-1}, \Sigma\right) \mid C_{v_{*}}^{1}, X_{1}=1\right]\right] \leq \frac{\left(v-b\left(v_{*}\right)\right) G\left(v_{*}\right)}{P\left(C_{v *}^{1}, X_{1}=1\right)} . \quad \text { Equation } 101 .
$$

Putting these two facts in one expression, we have

$$
\begin{array}{r}
\sup _{\hat{b} \in\left[b(0), b\left(v_{*}\right)\right) \cup\left\{b(v) \mid v=v_{*}, b(v)<\omega\right\}} E\left[\Pi_{1}\left(v, \hat{b}, b\left(\vec{V}_{-1}\right), X_{-1}, \Sigma\right) \mid C_{v_{*}}^{1}, X_{1}=1\right] \\
=\left\{\begin{aligned}
E\left[\Pi_{1}\left(v, b(v), b\left(\vec{V}_{-1}\right), X_{-1}, \Sigma\right) \mid C_{v_{*}}^{1}, X_{1}=1\right] & \text { if } v<v_{*} \\
\frac{\left(v-b\left(v_{*}\right)\right) G\left(v_{*}\right)}{P\left(C_{v *}^{1}, X_{1}=1\right)} & \text { if } v \geq v_{*} .
\end{aligned}\right.
\end{array}
$$

We next aim to show that $E\left[\Pi\left(v, b(v), b\left(\vec{V}_{-1}\right), X_{-1}, \Sigma\right) \mid C_{v_{*}}^{1}, X_{1}=1\right]>(v-\omega)=E\left[\Pi\left(v, \omega, b\left(\vec{V}_{-1}\right), X_{-1}, \Sigma\right) \mid C_{v_{*}}^{1}, X_{1}=1\right]$ for $v<v_{*}$. To that end, define a function $\tilde{\Delta}(v)$ given by

$$
\tilde{\Delta}(v)=\frac{(v-\tilde{b}(v)) G(v)}{P\left(C_{v_{*}}^{1} \mid X_{1}=1\right)}-(v-\omega)=\frac{1}{P\left(C_{v_{*}}^{1} \mid X_{1}=1\right)} \int_{0}^{v} G(y) d y-v+\omega .
$$

Equation 103. 
Note that for $v<v_{*}$

$$
\tilde{\Delta}^{\prime}(v)=\frac{1}{P\left(C_{v_{*}}^{1} \mid X_{1}=1\right)}\left(G(v)-P\left(C_{v_{*}}^{1} \mid X_{1}=1\right)\right)<0
$$

Equation 104.

where the last inequality is justified by Lemma 24. So $\tilde{\Delta}$ is decreasing on $\left[0, v_{*}\right]$ and $\tilde{\Delta}\left(v_{*}\right)=\Delta\left(v_{*}\right) \geq 0$ (due to the definition of $v_{*}$ and the fact that $b$ is increasing and continuous on $\left.\left[0, v_{*}\right]\right)$ which implies $\tilde{\Delta}(v)>0$ for $v<v_{*}$. Thus we indeed have

$$
E\left[\Pi_{1}\left(v, b(v), b\left(\vec{V}_{-1}\right), X_{-1}, \Sigma\right) \mid C_{v_{*}}^{1}, X_{1}=1\right]-E\left[\Pi_{1}\left(v, \omega, b\left(\vec{V}_{-1}\right), X_{-1}, \Sigma\right) \mid C_{v_{*}}^{1}, X_{1}=1\right]=\tilde{\Delta}(v)>0 \quad \text { Equation } 105 .
$$

for $v<v_{*}$. Thus, by Equation 102, Equation 98 holds when $v<v_{*}$. Next we consider the case $v=v_{*}$. If $b\left(v_{*}\right)<\omega$, then using Equation 102 and the fact that $\Delta\left(v_{*}\right) \geq 0$ we have

$$
\begin{aligned}
E\left[\Pi_{1}\left(v_{*}, b\left(v_{*}\right), b\left(\vec{V}_{-1}\right), X_{-1}, \Sigma\right) \mid C_{v_{*}}^{1}, X_{1}=1\right] & =\frac{\left(v_{*}-b\left(v_{*}\right)\right) G\left(v_{*}\right)}{P\left(C_{v_{*}}^{1}, X_{1}=1\right)} \\
& \geq(v-\omega) \\
& =E\left[\Pi_{1}\left(v_{*}, \omega, b\left(\vec{V}_{-1}\right), X_{-1}, \Sigma\right) \mid C_{v_{*}}^{1}, X_{1}=1\right], \quad \text { Equation } 106 .
\end{aligned}
$$

which by Equation 102 proves Equation 98 when $v=v_{*}$ and $b\left(v_{*}\right)<\omega$. Next we consider the case that $v=v_{*}$ and $b\left(v_{*}\right)=\omega$. Note that $\Delta(v)>0$ for $v<v_{*}$ and $\Delta\left(v_{*}\right) \leq 0$ by the definition of $v_{*}$ and the fact that $\Delta$ is increasing and continuous on $\left[0, v_{*}\right]$. So, by the same reasoning found in Proposition 25 we can see that both $v_{*}=1$ and $\Delta\left(v_{*}\right)=0$. Thus, if $b\left(v_{*}\right)=\omega$, then

$$
E\left[\Pi_{1}\left(v_{*}, b\left(v_{*}\right), b\left(\vec{V}_{-1}\right), X_{-1}, \Sigma\right) \mid C_{v_{*}}^{1}, X_{1}=1\right]=v_{*}-\omega=\frac{\left(v_{*}-b\left(v_{*}\right)\right) G\left(v_{*}\right)}{P\left(C_{v_{*}}^{1}, X_{1}=1\right)} .
$$

Equation 107.

So again by Equation 102 and the fact $b\left(v_{*}\right)=\omega$, the above proves Equation 98 when $v=v_{*}$ and $b\left(v_{*}\right)=\omega$. Finally, if $v>v_{*}$, we consider the function $\tilde{E}:[0,1] \rightarrow[0, \infty)$ defined by $\tilde{E}(x)=(v-\tilde{b}(x)) G(x) / P\left(C_{v_{*}}^{1} \mid X_{1}=1\right)=((v-x) G(x)+$ $\left.\int_{0}^{x} G(y) d y\right) / P\left(C_{v_{*}}^{1} \mid X_{1}=1\right)$. Note that (similar to above) $\tilde{E}^{\prime}(x)=(v-x) G^{\prime}(x)$ and so $\tilde{E}(x)$ takes a global maximum value at $x=v$. Using that fact, Equation 102, and the fact that $\Delta(v)<0$ (as $v>v_{*}$ ), we have

$$
\begin{aligned}
& \sup _{\hat{b} \in\left[b(0), b\left(v_{*}\right)\right) \cup\left\{b(v) \mid v=v_{*}, b(v)<\omega\right\}} E\left[\Pi_{1}\left(v, \hat{b}, b\left(\vec{V}_{-1}\right), X_{-1}, \Sigma\right) \mid C_{v_{*}}^{1}, X_{1}=1\right] \\
& =\left(v-b\left(v_{*}\right)\right) G\left(v_{*}\right) \\
& =\left(v-\tilde{b}\left(v_{*}\right)\right) G\left(v_{*}\right) \\
& =\tilde{E}\left(v_{*}\right) \\
& <\tilde{E}(v) \\
& =(v-\tilde{b}(v)) G(v)<(v-\omega) \\
& \left.=E\left[\Pi_{1}\left(v, b(v), b\left(\vec{V}_{-1}\right), X_{-1}, \Sigma\right) \mid C_{v_{*}}^{1}, X_{1}=1\right]\right] .
\end{aligned}
$$

Equation 108.

Thus, Equation 98 holds when $v>v_{*}$ and so Equation 98 holds for all $v \in[0,1]$, completing the proof.

\section{A MODEL WITH SIMULTANEOUS BIDDING}

One of the hallmarks of the SFPBO auction was the serial nature of the auction. The reason we considered bidders bidding in serial was to reflect how bidders bid on the Best Offer auction on eBay. In particular, if a bidder on eBay submits the BIN price, then that bidder will win the auction with certainty. However, often in auction theory auction models are created bidders submit their bids simultaneously and any resulting ties are broken randomly. For the second-price with buy-out auction considered in the work of Reynolds and Wooders this latter approach is used. ${ }^{3}$ Here we consider how, if at all, the permissible SBNE strategies for our SFPBO auction would change if we took this alternate approach. We will call the resulting model the First-Price Buy Out (FPBO) model and define it below: 
Definition 29. The conditions of the First Price Buy Out (FPBO) auction are conditions (i) through (xi) of the definition of the SFPBO auction (Definition 9) with the added condition

(xii) all bidders bid simultaneously.

Since the bidders in the FPBO bid simultaneously, all bidders have the opportunity to bid. Thus, we modify our definition of the SBNE for this model:

Definition 30. Assume the conditions of the FPBO auction. We say that $b:[0,1] \rightarrow[0, \omega]$ is a permissible symmetric Bayesian Nash equilibrium bidding strategy for the FPBO auction (or just permissible SBNE) if $b$ is permissible and for all $i \in\{1, \ldots, m+1\}$

$$
\sup _{\hat{b} \in[0, \omega]} E\left[\Pi_{i}\left(v_{i}, \hat{b}, b\left(\vec{V}_{-i}\right), \vec{X}_{-i}, \Sigma\right) \mid X_{i}=1\right]=E\left[\Pi_{i}\left(v_{i}, b\left(v_{i}\right), b\left(\vec{V}_{-i}\right), \vec{X}_{-i}, \Sigma\right) \mid X_{i}=1\right] .
$$

Equation 109.

We will derive for a SBNE for this FPBO auction. Since the derivation is similar to the SBNE for the SFPBO auction, we will omit many of the details of the derivation, focusing only on important differences.

For the SBNE for the FPBO auction, we no longer condition on the event $C_{v_{*}}^{i}$, which makes deriving the SBNE easier, but also means that a bidder who submits the maximum bid $\omega$ no longer wins the item with certainty. In fact, given a particular threshold $v_{*}$, if bidder $i$ bids $\omega$, then bidder $i$ will win if for all $j \in\{1, \ldots, m+1\}$, if $\Sigma(j)<\Sigma(i)$ then $X_{j}=0$ or $V_{j} \leq v_{*}$. That is, bidder $i$ wins in the case of the event $C_{v_{*}}^{i}$. More precisely, we have an analogue to Proposition 17:

Proposition 31. Assume the conditions of the FPBO auction. Let $b:[0,1] \rightarrow[0, \omega]$ be a permissible bidding function for the $F P B O$ auction with the cut-off value of $v_{*}$, and suppose that $b(0)=0$. Let $b^{-1}$ be the inverse of $\left.b\right|_{\left[0, v_{*}\right]}$ and fix $i \in\{1, \ldots, m+1\}$. If $v \in[0,1]$ and $\hat{b} \in[0, \omega]$, then

$$
E\left[\Pi_{i}\left(v, \hat{b}, b\left(\vec{V}_{-i}\right), \vec{X}_{-i}, \Sigma\right) \mid X_{i}=1\right]=\left\{\begin{aligned}
(v-\hat{b}) G\left(b^{-1}(\hat{b})\right) & \text { if } \hat{b} \leq b\left(v_{*}\right) \text { and } \hat{b}<\omega \\
(v-\hat{b}) G\left(v_{*}\right) & \text { if } b\left(v_{*}\right)<\hat{b}<\omega \\
(v-\omega) P\left(C_{v_{*}}^{1} \mid X_{1}=1\right) & \text { if } \hat{b}=\omega .
\end{aligned}\right.
$$

In particular, $E\left[\Pi_{i}\left(v, \hat{b}, b\left(\vec{V}_{-i}\right), \vec{X}_{-i}, \Sigma\right) \mid X_{i}=1\right]$ is independent of $i$.

We omit a proof of the above as the proposition can be proven analogously to the proof of Proposition 17. From Proposition 31, one can prove an analogue to Theorem 18:

Theorem 32. Assume the conditions of the FPBO auction. Let $b:[0,1] \rightarrow[0, \omega]$ be a permissible SBNE for the FPBO auction. Then, if $v \in\left[0, v_{*}\right]$

$$
b(v)=\tilde{b}(v)=\left\{\begin{aligned}
v-\frac{\int_{0}^{v} G(y) d y}{G(v)} & \text { if } 0<v \leq v_{*} \\
0 & \text { if } v=0 .
\end{aligned}\right.
$$

Again, we omit the proof as it is analogous to the proofs of Proposition 13, Proposition 14, and Theorem 18. Note that Theorem 32 implies that any SBNE to the FPBO auction must be the same as as the unique SBNE to the SFPBO auction before the cut-off value $v_{*}$. But must the cut-off value be the same? To answer this question, we need a new version of our difference function $\Delta$ :

Definition 33. Assume conditions (i) through (xi) of the SFPBO auction. Define the function $\bar{\Delta}:[0,1] \rightarrow \mathbb{R}$ by

$$
\bar{\Delta}(v):=(v-\tilde{b}(v)) G(v)-(v-\omega) P\left(C_{v}^{1} \mid X_{1}=1\right)=\int_{0}^{v} G(y) d y-(v-\omega) P\left(C_{v}^{1} \mid X_{1}=1\right)
$$

Equation 112. 
In light of Proposition 31 and Theorem 32, $\bar{\Delta}$ measures the difference in expected value of bidding using the SBNE without a cutoff $\tilde{b}$ and using the buy out price $\omega$. In the next proposition we state the obvious fact that $\Delta$ and $\bar{\Delta}$ are related, and thus many of the properties we proved about $\Delta$ carry over to $\bar{\Delta}$ :

Proposition 34. Assume conditions (i) through (xi) of the SFPBO auction. Then

$$
\bar{\Delta}(v)=P\left(C_{v}^{1} \mid X_{1}=1\right) \Delta(v) .
$$

Equation 113.

Furthermore, $\bar{\Delta}(0)>0$ and we have $\bar{\Delta}(v)=0 \Longleftrightarrow \Delta(v)=0$. Still furthermore, if $b$ is a permissible SBNE for the FPBO auction with associated cut-off value $v_{*}$, then $\bar{\Delta}(v)>0$ for $v \in\left(0, v_{*}\right)$ and $\bar{\Delta}\left(v_{*}\right) \geq 0$. If $v_{*}<1$ then $b\left(v_{*}\right)<\omega$ and $\bar{\Delta}\left(v_{*}\right)=0$.

Proof. Equation 113 follows directly from the definitions of $\Delta$ and $\bar{\Delta}$. Since $P\left(C_{v}^{1} \mid X_{1}=1\right)>0, \bar{\Delta}(v)=0 \Longleftrightarrow$ $\Delta(v)=0$ holds by Equation 113. $\bar{\Delta}(0)>0$ follows from Proposition 20 and Equation 113. The properties $\bar{\Delta}(v)>0$ for $v \in\left(0, v_{*}\right)$ and $\bar{\Delta}\left(v_{*}\right) \geq 0$ can be proven an analogously to Proposition 23. If $v_{*}<1$, then $b\left(v_{*}\right)<\omega$ follows from a proof analogous to that of Proposition 25. Finally $\bar{\Delta}\left(v_{*}\right)=0$ follows by a proof analogous to the proof of Proposition 26.

We can now state the main theorem of this section.

Theorem 35. Assume the conditions of the FPBO auction, suppose that there exists a SBNE $b:[0,1] \rightarrow[0, \omega]$ for this auction with corresponding value $v_{*}$. If $\bar{\Delta}(v) \geq 0$ for all $v \in[0,1]$, then

$$
b(v)=\left\{\begin{array}{rl}
0 & \text { for } v=0 \\
v-\frac{\int_{0}^{v} G(y) d y}{G(v)} & \text { for } 0<v
\end{array} .\right.
$$

Equation 114.

If $\bar{\Delta}(v)<0$ for some $v \in[0,1]$, then

$$
b(v)=\left\{\begin{array}{rl}
0 & \text { for } v=0 \\
v-\frac{\int_{0}^{v} G(y) d y}{G(y)} \quad & \text { for } 0<v \leq v_{*} . \\
\omega & \text { for } v>v_{*}
\end{array} .\right.
$$

Moreover, $v_{*}$ is the unique value in $[0,1]$ such that $\bar{\Delta}\left(v_{*}\right)=0$ and $b(v)$ is equal to the unique SBNE for the SFPBO auction.

Proof. First suppose $\bar{\Delta}(v) \geq 0$ for all $v \in[0,1]$. If $v_{*}<1$, then by Proposition $34 \bar{\Delta}\left(v_{*}\right)=0$. Again by Proposition 34 we thus know $\Delta\left(v_{*}\right)=0$ and since $\Delta$ is decreasing, we have $\Delta(1)<0$. So $\bar{\Delta}(1)=P\left(C_{1}^{1} \mid X_{1}=1\right) \Delta(1)<0$, which contradicts our assumption. Thus, $v_{*}=1$ and Equation 114 follows from Theorem 32. Next suppose $\bar{\Delta}(v)<0$ for some $v \in[0,1]$. If $v_{*}=1$, then by Proposition $34 \bar{\Delta}(v) \geq 0$ for all $v \in[0,1]$, a contradiction. Thus, $v_{*}<1$ and Equation 115 and the first assertion following it follow from Theorem 32 and Proposition 34. The last assertion follows from Equation 113 and Theorem 27.

Heuristically, this result is surprising. In the SFPBO auction, a bidder not only knows that she will win with certainly if she bids $\omega$, but that whatever bidders bid before her must have values less than the cut-off value. However, in equilibrium she behaves as she would in the FPBO auction. That is, in equilibrium in the SFPBO auction she behaves as if she did not have this extra information. As we noted (in more detail) in the comment following Proposition 17, this equivalency is due to the fact that if a bidder has a value under the cut-off value of $v_{*}$, she can only win if all bidders also have values under the cut-off value. 


\section{SELLER STRATEGY IN SETTING THE BUY-OUT PRICE}

In the SFPBO/FPBO auction model, the seller seems to do himself a disservice by putting a ceiling (the buy-out price $\omega$ ) on the highest possible bid. However, playing devil's advocate, one could dispute this heuristic point. Indeed, such a contrarian could point to the fact that the unique permissible SBNE can have a jump discontinuity, and thus in certain situations buyers could bid more in a SFPBO/FPBO auction than a standard first-price sealed-bid auction. Perhaps a seller could set a buyout price $\omega$ such that his expected revenue in this type of auction is the same or greater than the expected revenue in a straight first-price sealed-bid auction with no minimum bid (that is, an auction that does not have a ceiling on a high bid). Furthermore, because in the SFPBO/FPBO model the seller cannot set an open reserve price other than 0 , setting $\omega$ higher than any bidder is willing to pay is not an optimal auction. ${ }^{7}$ That said, we will now contradict that contrarian point of view and prove that setting $\omega$ higher than any bidder is willing to pay does generate the maximal expected revenue to the seller.

Recall that cut-off value $v_{*}$ is implicitly determined by the equation $\Delta(v)=0$ (as long as $v_{*}<1$ ) and $\Delta$ depends on $\omega$. Solving the equation $\Delta\left(v_{*}\right)=0$ for $\omega$ yields

$$
\omega=v_{*}-\frac{\int_{0}^{v_{*}} G(y) d y}{P\left(C_{v_{*}}^{1} \mid X_{1}=1\right)}
$$

Equation 116.

If $v_{*}=1$, then the bidders always use the strategy $\tilde{b}$ and never submit the buy-out price of $\omega=1-\frac{\int_{0}^{1} G(y) d y}{P\left(C_{1}^{1} \mid X_{1}=1\right)}$. Setting $\omega$ higher than $1-\frac{\int_{0}^{1} G(y) d y}{P\left(C_{1}^{1} \mid X_{1}=1\right)}$ would have the same effect of bidders never submitting the buy-out price as a bid, so we can find an optimal cut-off value $v_{*}$ and use it to determine a corresponding optimal $\omega$.

Theorem 36. Assume the conditions (i) through (xi) of the SFPBO auction. For any $v_{*} \in[0,1]$, let $b_{v_{*}}(v)$ be the SBNE of the $S F P B O$ auction as defined in Theorem 28 with the corresponding cut-off value of $v_{*}$. Let $R_{v_{*}}=b_{v_{*}}(\tilde{Y})$ be the revenue generated by the SFPBO auction with $\omega=v_{*}-\frac{\int_{0}^{v_{*}} G(y) d y}{P\left(C_{v_{*}}^{1} \mid X_{1}=1\right)}$. The quantity $E\left[R_{v_{*}}\right]$ is maximized at $v_{*}=1$.

Proof. First we derive a formula for the $\operatorname{cdf} \tilde{G}(y)$ of $\tilde{Y}=\max _{1 \leq j \leq m+1} X_{j} V_{j}$. We have

$$
\tilde{G}(y)=P(Y \leq y)=\sum_{n=0}^{m+1} P(Y \leq y \mid N=n) P(N=n)=\sum_{n=0}^{m+1} F(y)^{n} \tilde{q}_{n},
$$

Equation 117.

as $\tilde{q}_{n}=P(N=n)$ by definition of $\tilde{q}_{n}$. As our formula for $E\left[R_{v_{*}}\right]$ will involve both $\tilde{q_{n}}$ 's (from $\tilde{G}$ ) and $q_{n}$ 's (from $G$ ), we will want a relationship between the two quantities. To that end, for any $n \in\{2, \ldots, m+1\}$ we have

$$
\begin{aligned}
q_{n} & =P\left(N=n+1 \mid X_{1}=1\right) \\
& =\frac{1}{P\left(X_{1}=1\right)} P\left(N=n+1, X_{1}=1\right) \\
& =\frac{1}{P\left(X_{1}=1\right)} \sum_{\vec{\beta} \in\{0,1\}^{m}, \sum_{i=1}^{m} \beta_{i}=n} P\left(X_{1}=1, X_{2}=\beta_{1}, X_{3}=\beta_{2}, \ldots, X_{m+1}=\beta_{m}\right) \\
& =\frac{1}{P\left(X_{1}=1\right)} \sum_{\vec{\beta} \in\{0,1\}^{m}, \sum_{i=1}^{m} \beta_{i}=n} P\left(X_{1}=1, X_{2}=1, \ldots, X_{n+1}=1, X_{n+2}=0, \ldots, X_{m+1}=0\right) \\
& =\frac{1}{P\left(X_{1}=1\right)}\left(\begin{array}{c}
m \\
n
\end{array}\right) P\left(X_{1}=1, X_{2}=1, \ldots, X_{n+1}=1, X_{n+2}=0, \ldots, X_{m+1}=0\right) \\
& =\frac{1}{P\left(X_{1}=1\right)} \frac{\left(\begin{array}{c}
m \\
n
\end{array}\right)}{\left(\begin{array}{c}
m+1 \\
n+1
\end{array}\right)} \\
& =\frac{1}{P\left(X_{1}=1\right)} \frac{n+1}{m+1} \tilde{q}_{n+1} .
\end{aligned}
$$

Equation 118. 
Now we can start to compute $E\left[R_{v_{*}}\right]$ :

$$
\begin{aligned}
E\left[R_{v_{*}}\right] & =E\left[b_{v_{*}}(Y)\right] \\
& =\int_{0}^{1} b_{v_{*}}(y) \tilde{G}^{\prime}(y) d y \\
& =\int_{0}^{v_{*}} \tilde{b}(y) \tilde{G}^{\prime}(y) d y+\int_{v_{*}}^{1} \omega \tilde{G}^{\prime}(y) d y \\
& =\int_{0}^{v_{*}} \tilde{b}(y) \tilde{G}^{\prime}(y) d y+\omega\left(1-\tilde{G}\left(v_{*}\right)\right) \\
& =\int_{0}^{v_{*}}\left(y-\frac{\int_{0}^{y} G(x) d x}{G(y)}\right) \tilde{G}^{\prime}(y) d y+\left(v_{*}-\frac{\int_{0}^{v_{*}} G(y) d y}{P\left(C_{v_{*}}^{1} \mid X_{1}=1\right)}\right)\left(1-\tilde{G}\left(v_{*}\right)\right) . \quad \text { Equation } 119 .
\end{aligned}
$$

In the last line of Equation 119, the quantity $\frac{\tilde{G}^{\prime}(y)}{G(y)}$ appears. To move forward, we first simplify that quotient using Equation 118:

$$
\begin{aligned}
\frac{\tilde{G}^{\prime}(y)}{G(y)} & =\frac{\sum_{n=0}^{m}(n+1) F(y)^{n} f(y) \tilde{q}_{n+1}}{\sum_{n=0}^{m} F(y)^{n} q_{n}} \\
& =(m+1) P\left(X_{1}=1\right) f(y) \frac{\sum_{n=0}^{m} F(y)^{n} q_{n}}{\sum_{n=0}^{m} F(y)^{n} q_{n}} \\
& =(m+1) P\left(X_{1}=1\right) f(y) .
\end{aligned}
$$

Equation 120.

Plugging Equation 120 into Equation 119 and integrating by parts in the second integral of the second line yields

$$
\begin{aligned}
E\left[R_{v_{*}}\right]= & \int_{0}^{v_{*}}\left(y-\frac{\int_{0}^{y} G(x) d x}{G(y)}\right) \tilde{G}^{\prime}(y) d y+\left(v_{*}-\frac{\int_{0}^{v_{*}} G(y) d y}{P\left(C_{v_{*}}^{1} \mid X_{1}=1\right)}\right)\left(1-\tilde{G}\left(v_{*}\right)\right) \\
= & \int_{0}^{v_{*}} y \tilde{G}^{\prime}(y) d y-(m+1) P\left(X_{1}=1\right) \int_{0}^{v_{*}}\left(\int_{0}^{y} G(x) d x\right) f(y) d y+\left(v_{*}-\frac{\int_{0}^{v_{*}} G(y) d y}{P\left(C_{v_{*}}^{1} \mid X_{1}=1\right)}\right)\left(1-\tilde{G}\left(v_{*}\right)\right) \\
= & \int_{0}^{v_{*}} y \tilde{G}^{\prime}(y) d y-(m+1) P\left(X_{1}=1\right)\left(\left(F(y) \int_{0}^{y} G(x) d x\right)_{y=0}^{y=v_{*}}-\int_{0}^{v_{*}} F(y) G(y) d y\right)+ \\
& \left(v_{*}-\frac{\int_{0}^{v_{*}} G(y) d y}{P\left(C_{v_{*}}^{1} \mid X_{1}=1\right)}\right)\left(1-\tilde{G}\left(v_{*}\right)\right) \\
= & \int_{0}^{v_{*}} y \tilde{G}^{\prime}(y) d y-(m+1) P\left(X_{1}=1\right)\left(\left(F\left(v_{*}\right) \int_{0}^{v_{*}} G(y) d y\right)-\int_{0}^{v_{*}} F(y) G(y) d y\right)+ \\
& v_{*}\left(1-\tilde{G}\left(v_{*}\right)\right)-\frac{1-\tilde{G}\left(v_{*}\right)}{P\left(C_{v_{*}}^{1} \mid X_{1}=1\right)} \int_{0}^{v_{*}} G(y) d y
\end{aligned}
$$

To push the calculation of $E\left[R_{v_{*}}\right]$ forward, we next we simplify the quotient $\frac{1-\tilde{G}\left(v_{*}\right)}{P\left(C_{v_{*}}^{1} \mid X_{1}=1\right)}$. If $v_{*}=1$, then the quotient is 0 . 
If $v_{*}<1$ we have:

$$
\begin{aligned}
\frac{1-\tilde{G}\left(v_{*}\right)}{P\left(C_{v_{*}}^{1} \mid X_{1}=1\right)} & =\frac{\left(1-\sum_{n=0}^{m+1} F\left(v_{*}\right)^{n} \tilde{q}_{n}\right)\left(1-F\left(v_{*}\right)\right)}{\sum_{n=0}^{m} \frac{1}{n+1}\left(1-F\left(v_{*}\right)^{n+1}\right) q_{n}} \\
& =\frac{\left(1-\tilde{q}_{0}-\sum_{n=0}^{m} F\left(v_{*}\right)^{n+1} \tilde{q}_{n+1}\right)\left(1-F\left(v_{*}\right)\right)}{\sum_{n=0}^{m} \frac{1}{n+1}\left(1-F(v)^{n+1}\right) q_{n}} \\
& =\frac{\left(\sum_{n=1}^{m+1} \tilde{q}_{n}-\sum_{n=0}^{m} F\left(v_{*}\right)^{n+1} \tilde{q}_{n+1}\right)\left(1-F\left(v_{*}\right)\right)}{\sum_{n=0}^{m} \frac{1}{n+1}\left(1-F(v)^{n+1}\right) q_{n}} \\
& =\frac{\left(\sum_{n=0}^{m}\left(1-F\left(v_{*}\right)^{n+1}\right) \tilde{q}_{n+1}\right)\left(1-F\left(v_{*}\right)\right)}{\sum_{n=0}^{m} \frac{1}{n+1}\left(1-F(v)^{n+1}\right) q_{n}} \\
& =\frac{(m+1) P\left(X_{1}=1\right)\left(\sum_{n=0}^{m} \frac{1}{n+1}\left(1-F\left(v_{*}\right)^{n+1}\right) q_{n}\right)\left(1-F\left(v_{*}\right)\right)}{\sum_{n=0}^{m} \frac{1}{n+1}\left(1-F(v)^{n+1}\right) q_{n}} \\
& =(m+1) P\left(X_{1}=1\right)\left(1-F\left(v_{*}\right)\right) .
\end{aligned}
$$

Equation 122.

Since $(m+1) P\left(X_{1}=1\right)(1-F(1))=0$, we have $\frac{1-\tilde{G}\left(v_{*}\right)}{P\left(C_{v_{*}}^{1} \mid X_{1}=1\right)}=(m+1) P\left(X_{1}=1\right)\left(1-F\left(v_{*}\right)\right)$ for all $v_{*}$. Thus, we can continue Equation 121:

$$
\begin{aligned}
E\left[R_{v_{*}}\right] & =\int_{0}^{v_{*}} y \tilde{G}^{\prime}(y) d y-(m+1) P\left(X_{1}=1\right)\left(F\left(v_{*}\right) \int_{0}^{v_{*}} G(y) d y-\int_{0}^{v_{*}} F(y) G(y) d y\right)+ \\
& v_{*}\left(1-\tilde{G}\left(v_{*}\right)\right)-\frac{1-\tilde{G}\left(v_{*}\right)}{P\left(C_{v_{*}}^{1} \mid X_{1}=1\right)} \int_{0}^{v_{*}} G(y) d y \\
& =\int_{0}^{v_{*}} y \tilde{G}^{\prime}(y) d y+v_{*}\left(1-\tilde{G}\left(v_{*}\right)\right)-(m+1) P\left(X_{1}=1\right)\left(\int_{0}^{v_{*}} G(y) d y-\int_{0}^{v_{*}} F(y) G(y) d y\right) . \quad \text { Equation } 123 .
\end{aligned}
$$

Taking the derivative of the above yields

$$
\begin{aligned}
\frac{1}{(m+1) P\left(X_{1}=1\right)} \frac{d}{d v_{*}} E\left[R_{v_{*}}\right] & =\frac{1}{(m+1) P\left(X_{1}=1\right)}\left(v_{*} \tilde{G}^{\prime}\left(v_{*}\right)+\left(1-\tilde{G}\left(v_{*}\right)\right)-v_{*} \tilde{G}^{\prime}\left(v_{*}\right)\right)-\left(1-F\left(v_{*}\right)\right) G\left(v_{*}\right) \\
& =\frac{1-\tilde{G}\left(v_{*}\right)}{(m+1) P\left(X_{1}=1\right)}-\left(1-F\left(v_{*}\right)\right) G\left(v_{*}\right) \\
& =\left(1-F\left(v_{*}\right)\right)\left(P\left(C_{v_{*}}^{1} \mid X_{1}=1\right)-G\left(v_{*}\right)\right) \\
& >0
\end{aligned}
$$

for $v_{*} \in(0,1)$. We used Equation 122 in the last equality and Lemma 24 in the last inequality. Thus, $E\left[R_{v_{*}}\right]$ is increasing in $v_{*}$ and thus must be maximized at the right endpoint $v_{*}=1$, completing the proof.

By Theorem 36, a seller should set $\omega$ high enough so that $v_{*}=1$. By setting $v_{*}=1$ in Equation 116, any $\omega$ bigger than or equal to $1-\frac{\int_{0}^{1} G(y) d y}{P\left(C_{1}^{1} \mid X_{1}=1\right)}=1-\int_{0}^{1} G(y) d y$ would do that job. In this case, the seller has set $\omega$ high enough so that in equilibrium no bidder (except possibly a bidder with $v=1$ in the case $\omega=1-\int_{0}^{1} G(y) d y$ ) will ever use the buy-out price $\omega$, making the bidders behave in equilibrium in both the SFPBO and FPBO auctions as they would in a first-price auction with no reserve price and without a buy-out price.

\section{SUMMARY OF FINDINGS AND FUTURE WORK}

Here we take a heuristic account of our results. In the Secret Threshold (ST) game, we assume risk-neutral, independent, and symmetric bidders act in serial, either submit one take-it-or-leave-it offer to the seller or submit a buy-out price, and 
know the previous number of failed bids. The seller accepts any bid that exceeds some secret threshold (or secret reserve) price as well as the buy-out price. We derived optimal bids for this game that are based on the bidder's value for the object as well as the number of previous failed bids (Theorem 4 and Theorem 5). We also show that given a fixed value and assuming bidders bid optimally, the expected bidder surplus decreases in the number of previous failed bids (Theorem 8). Thus, it is better to be an early bidder rather than a late bidder in the ST game, despite the fact that late bidders have more information than early bidders.

In the Serial First-Price Buy-Out (SFPBO) auction, we again assume risk-neutral, independent, and symmetric bidders act in serial and submit one offer to the seller that is at or below the seller's buy-out price. However, in this auction the seller does not have a secret threshold and will instead select the highest bid among the bidders. The auction immediately ends when one bidder submits the buy-out price for her with the buy-out bidder winning the auction and paying the buy-out price. Furthermore, at the time of bidding, bidders do not know how many previous failed bids have been made or how many bids will be made in the future, but do know that any previous bid must have been lower than the buy-out price. We found an equilibrium cut-off bidding strategy for this auction that is unique up to some reasonable conditions (Theorem 28). Furthermore, we found that if we changed the SFPBO auction to an auction where bidders acted simultaneously and ties were broken randomly (the First-Price Buy-Out (FPBO) auction), then any reasonable equilibrium cut-off bidding strategy for the FPBO auction must be the same strategy as the equilibrium strategy for the SFPBO auction (Theorem 35). This last result is surprising: bidders in the SFPBO auction have more information than bidders in the FPBO auction and yet they behave the same in equilibrium. Lastly, we considered how the seller could optimally set the buy-out price in either the SFPBO or FPBO auctions, and found that the seller should set the buy-out price higher than any bidder in equilibrium would consider submitting (Theorem 36). That is, the seller should set a buy-out price so high that the auction is effectively just a standard first-price sealed-bid auction with no reserve price.

One obvious question stemming from this last result is why sellers would choose to sell their goods using a Best Offer auction if the SFPBO and FPBO auctions models offer no more expected revenue than a standard first-price sealed-bid auction with no reserve, an auction that is not typically an optimal auction. An equally obvious possible explanation is that bidders are actually risk-adverse and not risk-neutral, and perhaps the SFPBO/FPBO auctions actually can generate more expected revenue than the first-price auction when bidders are risk-adverse. The risk-adverse assumption is made in Reynolds and Wooders and they show that revenue can be increased with buy-out prices in second-price auction, ${ }^{3}$ and thus risk aversion would be a natural assumption to include in future work on deriving equilibrium strategies for first-price with buy-out auctions. In fact, one could then compare the revenue to the second-price with buy-out auction modeled by Reynolds and Wooders with revenue to the first-price with buy-out. In light of our result that the SFPBO and FPBO models have the same equilibria, it would make sense to use the easier FPBO model, particularly since random tie-breaking for bidders using the buy-out price is the assumption found in Reynolds and Wooders work. Perhaps this future work could illuminate why the Buy-It-Now with Best Offer buying format is a popular way for sellers to sell goods on eBay.

\section{REFERENCES}

1. Hasker, K. and Sickles, R. (2010) eBay in the economic literature: analysis of an auction marketplace, Rev Ind Organ 37, $3-42$.

2. Steiglitz, K. (2007) Snipers, Shills, and Sharks: eBay and Human Behavior, Princeton University Press, Princeton.

3. Reynolds, S.S. and Wooders, J. (2009) Auctions with a buy price, Econ. Theory 38, 79-101.

4. McAfee, R. P. and McMillan, J. (1987) Auctions with a stochastic number of bidders, J. Econ. Theory 43, 1-19.

5. Krishna, V. (2002) Auction Theory 1st ed., Academic Press, San Diego.

6. Menezes, F. M. and Monteiro, P. K. (2005) An Introduction to Auction Theory, Oxford University Press, New York.

7. Myerson, R. (1981) Optimal Auction Design, Mathematics of Operations Research 6, 58-73. 


\section{ABOUT THE STUDENT AUTHORS}

William J. Britt graduated from Muhlenberg College in May of 2017 as a mathematics and and economics double major. He was a member of Muhlenberg varsity football team and a captain. Will plans to pursue an actuarial career.

Jamie A. Oliva graduated from Muhlenberg College in May of 2016 with honors in mathematics and became a postbaccalaureate student at Smith College in mathematics. She planned to pursue a Ph.D. in mathematics following her time at Smith, but tragically passed away on Thanksgiving in 2016.

Brittney N. Tuff graduated from Muhlenberg College in May of 2016 as a mathematics and physical science double major and was the class salutatorian. She is a member of Phi Beta Kappa and a recipient of the Wesley S. Mitman Mathematical Prize. Brittney is now a software engineer at Elsevier.

Charli E. White graduated from Muhlenberg College in May of 2017 with a B.S. in mathematics. She is an experienced buyer and seller of musical memorabilia on eBay, and is currently pursuing a career in actuarial science at MetLife.

\section{PRESS SUMMARY}

Have you ever gone on eBay and wondered how much you should bid? This can be a tricky question, especially for a "Buy-It-Now or Best Offer" auction. Should you buy-it-now or make an offer? If you make an offer, how much should you offer? You want to make an offer that is large enough to have a good chance to win the item for but not too large since you want to get a good deal. In this paper we theoretically answer this question. Under certain assumptions, we mathematically determine when to buy-it-now, when to make an offer, and how much that offer should be. 


\title{
The Relationship Between Parenting Styles and Substance Use Among University Students
}

\author{
Zackaria I. Niari $i^{* a,}$, Danielle Dicke, \\ ${ }^{a}$ Department of Biology, Virginia Commonwealth University, Richmond, $V A$ \\ ${ }^{b}$ Department of Psychology, Virginia Commonwealth University, Richmond, VA \\ 'Department of Human and Molecular Genetics, Virginia Commonwealth University, Richmond, VA \\ ${ }^{d}$ College Behavioral and Emotional Health Institute, Virginia Commonwealth University, Richmond, VA \\ ${ }^{e}$ Virginia Institute for Psychiatric and Behavioral Genetics, Virginia Commonwealth University, Richmond, VA \\ Student:niazizi@vcu.edu* \\ Mentors:cookem@vcu.edu*,dickdm@vcu.edu,adkinsae@vcu.edu
}

\begin{abstract}
Parenting styles are important in the behavioral development of adolescents. The environment created by the parent, in regards to communication with their child and level of independence given to their child, may influence the child's susceptibility to risk behaviors. This study examines the relationship between parenting style and substance use among university students. We hypothesized that university students exposed to lower levels of autonomy granting (AG) or parental involvement (PI) parenting styles would have an increased likelihood of alcohol and nicotine use. We also hypothesized that religiosity, parental education level, ethnicity, and gender would act as moderators of parenting styles and alcohol and nicotine use. Data from a diverse university-wide sample was collected in the fall semester of the student's freshman year from 2011-2014 $(N=9889,61.5 \%$ female). Results demonstrated that AG had a significant, negative association with alcohol use $(B=-0.033, p=0.006)$ and nicotine use $(B=-0.066, p<0.001)$. All moderators were found to be significant predictors of alcohol use, however only father education level demonstrated a borderline significant moderation of the relationship between PI and alcohol use. Religiosity, Black race, Asian race, and gender were found to be significant predictors of nicotine use. Only gender moderated the association between PI and nicotine use. Even though alcohol and nicotine use and AG were associated, our results indicate that once students enter university, previous parenting style does not have a strong effect on alcohol and nicotine use behaviors in our sample.
\end{abstract}

\section{KEYWORDS}

Parenting Styles; University Students; Risky Behaviors; Autonomy Granting; Parental Involvement; Alcohol; Nicotine; Drug; Behavioral Biology; Substance Use

\section{INTRODUCTION}

Frequent alcohol and nicotine use has been found to have negative consequences in terms of physical health, mental health, and social relationships., 2 Nicotine use has been shown to have negative effects on the physical health of individuals, such as heart problems and lung cancer. ${ }^{1}$ In addition, alcohol use among university students can lead to a range of consequences such as academic impairment, blackouts, sexual coercion/rape victimization, property damage, fights, and vehicular related death.3,4 In order to form better intervention and education plans, it is important to understand and explore the pathways to university student risk behaviors.

Parenting styles are important in the behavioral development of adolescents. It has been shown that parenting style could play a role in whether or not adolescents and college students are willing to participate in risky behaviors, such as alcohol use, nicotine use, and illicit drug use. ${ }^{5,6}$ Lack of communication between parent and adolescent is one of the factors in the development of risk behaviors and substance abuse. ${ }^{5}$ Studies have shown that certain parenting styles can influence issues such as self-concealment, resulting in the adolescent having less self-regulatory resources to maintain control of drinking. ${ }^{7}$ The diminished self-regulation can lead to impaired control over alcohol consumption and alcohol-related problems. ${ }^{7}$ Presence of an authoritative mother is directly linked to more secrets between the parent and adolescent, while having an authoritative father is directly linked to fewer secrets. ${ }^{7}$

As discussed above, previous studies have shown that parenting styles may influence the development of risk behaviors in adolescents. ${ }^{5,8}$ Therefore, while it is clear that parenting styles play a large role in adolescent behavior, far less is known regarding the relationship between parenting style and substance use in university students/emerging adulthood. The transition from high school to university is a key developmental milestone and has the potential for change in behavior and personal growth. ${ }^{6}$ This is primarily because university students, who generally live on campus in dormitories or on their own, are in an environment where parental supervision is limited while opportunities to engage in risky behaviors are abundant. ${ }^{6}$ Some of the risky behaviors university students can partake in include alcohol use, tobacco use, and illicit drug use. ${ }^{9,7}$ The transition to university, in 
combination with parenting style, could result in increased risk behaviors among freshman in a university setting and thus emphasize the importance of examining these relationships.

There are several other family level factors that could affect the relationship between parenting and substance use, such as religiosity, gender, ethnicity, parents' level of education. Some religions preach against the use of substances such as alcohol, nicotine, and marijuana because it is viewed as defiling of one's body and considered sinful. ${ }^{10} \mathrm{In}$ a study of university students in Northern Ireland, Wales and England, it was found that religiosity and abstaining from drug use were positively associated. ${ }^{9}$ Therefore, it is important to examine whether religiosity may play a role in freshman university student substance use outcomes. Parents' level of education is important in that it is usually negatively associated with financial stress. ${ }^{11}$ Occasional drug use has been known to be prevalent among students who have financial burdens and economic instability. ${ }^{9}$ This prevalence could be a result of environmental factors influenced by socio-economic status, such as home and school environment. ${ }^{11}$ Gender of the university student is important when examining parent-child dynamics; when the parent is the same sex as the subject, parenting style is found to be significantly related to self-regulation. This is crucial because self-regulation is found to be protective against alcohol use and abuse. ${ }^{8}$ A permissive parent, of the same sex as the subject, is found to be negatively associated with good selfregulatory processes for both men and women, while an authoritative parent, of the same sex as the subject, is found to be associated with positive levels of self-regulation among the subjects. ${ }^{8}$ In addition, previous studies have demonstrated that drinking patterns and risky drinking differ among ethnic groups and genders. ${ }^{12}$

While there are various types of parenting styles, from authoritative parenting to neglectful parenting; ${ }^{13}$ the current study focuses on the Steinberg Parenting styles. Autonomy Granting (AG) is a form of parenting in which parents encourage their kids to express their own ideas and thoughts without expressing psychological control and disapproval. ${ }^{14}$ Another dimension of parenting is Parental Involvement (PI), which entails the involvement of the parent within school functions and their awareness of the adolescent's peers, feelings, and interests. ${ }^{14}$ The lack of or limited exposure to either parenting style indicates a form of parenting in which the adolescent's views and opinions are not heard and there is little involvement invested in the adolescent.

The current study aims to examine whether there is a connection between parenting style and university student alcohol use or nicotine use. In addition, it seeks to test whether there are associations of alcohol and nicotine use with religiosity, ethnicity, gender, and education level of parents. Our study hypothesis is that if parents of a university student exhibit a lower degree of PI and AG, then there will be an increase in the frequency of alcohol use or nicotine use by the university student. If such a relationship between parenting style and university student alcohol/nicotine use exists, then we hypothesize that the relationship will be stronger for those with low religiosity and parent education level, and it will be stronger for males and minority groups.

\section{METHODS AND PROCEDURES}

\section{Sample}

The sample for this study is derived from the Spit for Science research project, IRB permission number: HM13352. This project is a university-wide research study, at a large, urban university, that focuses on understanding the genetic and environmental influences on the development of risk behaviors in college students. ${ }^{15}$ The majority of students reported that they live in oncampus housing $(88.1 \%)$. Programs organized by the residential life and housing are available. Freshman students, ages 18 or older, were recruited in the Fall from 2011 to 2014 by e-mail invitation. ${ }^{15}$ After consenting to participate online, students completed a survey containing questions that assessed personality and behavior, as well as family environment and experiences growing up. After completion of the survey, participants collected $\$ 10$ as payment and were given the option to submit their saliva sample for a second payment of $\$ 10 . .^{15}$

Data from 9889 participants were used from the fall cohorts of 2011, 2012, 2013, and 2014. Of the participants, 6040 were female $(61.5 \%)$. The mean age of the students was $18.50(\mathrm{SD}=0.43)$. Of the participants, 6114 reported that neither of their biological parents had a drinking problem $(72.4 \%)$. The ethnic breakdown of the participants was: White $(N=4881,50.1 \%)$, Black/African American $(N=1873,19.2 \%)$, Asian $(N=1615,16.6 \%)$, multiracial $(N=617,6.3 \%)$, Hispanic/Latino $(N=594,6.1 \%)$, Native Hawaiian/Pacific Islander $(N=67,0.7 \%)$, American Indian/Native Alaskan $(N=51,0.5 \%)$. The ethnic breakdown was representative of the university's population.

\section{Protocol}

Study data were collected using REDCap (Research Electronic Data Capture) tools hosted at Virginia Commonwealth University. ${ }^{16}$ This secure, web-based application is designed to support data capture for research studies.

\section{Measures}

Parenting style was measured by a set of six statements, as obtained from Steinberg et al. 1992 $2^{14}$, with a four-point response scale of: "Strongly Agree," "Agree Somewhat," "Disagree Somewhat," and "Strongly Disagree."

Statements measuring the parent's AG included: "My parents said that I should give in on arguments rather than making people angry," "My parents told me that their ideas were correct and that I should not question them," and "My parents acted cold and unfriendly if I did something they didn't like." Statements measuring PI included: "My parents helped me with my school work if 
there was something I didn't understand," "My parents knew who my friends were," and "My parents spend time just talking with me."

Separate sum scores, with a range of 3-12, were created for the AG and PI parenting styles to measure the degree of the parenting styles. Variables were then mean centered.

Biological parent drinking problem was measured by two questions: "Do you think your biological mother has ever had a drinking problem?" and "Do you think your biological father has ever had a drinking problem?" Drinking problem was defined as "...his/her drinking caused problems at home, at work, with his/her health, with the police, or that he/she received alcohol treatment." Responses were a binary "No," or "Yes."

The biological parent drinking problem variable was created by considering the data from both questions to assess the percentage of students that reported neither of their biological parents having drinking problems.

Alcohol use was measured by questions based off the AUDIT-C. ${ }^{17}$ Alcohol use frequency was measured by the question "How often do you have a drink containing alcohol?" with a four-point response scale of: "Never," "Monthly or less," " 2 to 4 times a month," "2 to 3 times a week," and "4 or more times a week." The response "Never" was recoded as 0 days per month, "Monthly or less" as 1 day per month, "2 to 4 times a month" as 3 days per month, "2 to 3 times a week" as 10 days per month, and " 4 or more times a week" as 16 days per month.

Alcohol use quantity was measured by the question "How many drinks containing alcohol do you have on a typical day when you are drinking?" with a five-point response scale of: " 1 or 2 ," "3 or 4," " 5 or 6, , "7, 8, or 9," and "10 or more." The response " 1 or 2" was recoded as 1.5 drinks, " 3 or 4 " as 3.5 drinks, " 5 or 6" as 5.5 drinks, “7, 8, or 9" as 8 drinks, and " 10 or more" as 10 drinks. The alcohol variable was created by multiplying the recoded data from both questions to assess the quantity of drinks per month. The variable underwent log transformation to better approximate a normal distribution.

Nicotine use was measured by questions based off the IMPACTS study at University of Missouri. ${ }^{18}$ Nicotine use was measured by the question "How frequently did you smoke [cigarettes, cigars, smokeless tobacco products, or hookah] in the last 30 days?" with a seven-point response scale of: "I didn't smoke any [cigarettes, cigars, smokeless tobacco products, or hookah] in the last month," "once or twice," "A few days (3 to 4 days a month)," "A couple of days a week (5 to 11 days a month)," "Three times a week (12 to 14 days a month)," "Most days of the week (15 to 25 days a month)," and "Daily or almost daily (26 to 30 days a month)."

Four questions, with the same responses, regarding nicotine use in different forms (i.e. cigarette, cigars, smokeless tobacco, and hookah) were combined by selecting the highest use of each nicotine product to evaluate overall nicotine use.

Gender was measured by the binary response of: "Male," and "Female."

Ethnicity was measured by the question "Which one of these groups best describes you?" with an eight-point response scale of: "American Indian/Alaska Native," "Asian," "Black/African American," "Hispanic/Latino," "More than one race," "Native Hawaiian/Other Pacific Islander," "Unknown," and "White."

A binary variable of White and Black, White and Asian, and White and Hispanic/Latino was created to analyze ethnicity with white as the reference group.

Religiosity was measured by two questions, as obtained from Kendler et al. 1997:19 "In general, how important are your religious and spiritual beliefs in your daily life?" and "When you have problems or difficulties in your family, work, or personal life, how often do you seek spiritual comfort?”

Responses for the first question were on a four-point scale of: "Very important," "Somewhat important," "Not very important," and "Not at all important." Responses for the second question were on a four-point scale of: "Almost always," "Sometimes," "Rarely," and "Never."

A combined sum score, of a range of 2-8, was created to determine the degree of religiosity. Variables were then mean centered. Education level of parents was measured by two questions: "The woman who functioned as mother; How far in school did she go?" and "The man who functioned as father; How far in school did he go?"

Responses in regards to mother/father's level of education were based on 12 possible answer choices that were recoded. The response "He/She never went to school," was recoded as 0 years. Responses "He/She went to school, but I don't know what level," and "There was no one who functioned as a mother/father in my household" was recoded as missing. "Eighth grade or less" was recoded as 8 years. "More than eighth grade, but did not graduate from high school," and "Went to business, trade or vocation school instead of high school" were recoded as 10 years. "High school graduate," and "Completed a GED" were recoded as 12 years. "Went to business, trade, or vocational school after high school” was recoded as 13 years. "Went to college, but did not graduate" was recoded as 14 years. "Graduated from a college or university" was recoded as 16 years. "Professional training beyond a four-year college or university" was recoded as 20 years. The variable was then centered.

\section{Statistical Analysis}

Statistical analysis was conducted using the $22^{\text {nd }}$ version of the IBM SPSS Software. Linear regressions were used to analyze the relationship between AG and alcohol use, and between PI and alcohol use. Ordinal regressions were used to analyze the relationship between AG and nicotine use, and PI and nicotine use. Ordinal regressions were used for determining the relationship with nicotine use because of the ordinal nature of the nicotine use data. 
Interaction variables were created by cross-multiplying parenting styles with each moderator. The interaction variables were then used in a regression model to determine if the moderators interacted with each parenting style to produce an effect on nicotine or alcohol use. Linear regressions were conducted to determine the relationship between the moderators, biological parent drinking problem, and alcohol use, while ordinal regressions were conducted to determine the relationship between the moderators and nicotine use. To better gain an understanding of the relationship between the covariates and alcohol and nicotine use, linear regression analysis and ordinal regression analysis were conducted respectively.

\section{RESULTS}

\section{Sample Overview}

The participants in our sample had an average of 14.12 drinks per month. On average, participants used nicotine products one to four days a month. Table 1 below presents the mean, standard deviations, and sample size of the other variables.

\begin{tabular}{|c|c|c|c|c|}
\hline Measure & $n$ & Mean & Standard Deviation & Percent Endorsed \\
\hline Age & 7902 & 18.5 & 0.43 & \\
\hline \multicolumn{5}{|l|}{ Parenting Style } \\
\hline Autonomy Granting & 7369 & 8.17 & 2.18 & \\
\hline Parental Involvement & 7399 & 9.61 & 2.08 & \\
\hline Frequency of Alcohol Use & 4240 & 14.12 & 23.35 & \\
\hline Frequency of Nicotine Use & 7077 & 2.14 & 1.74 & \\
\hline Religiosity & 9193 & 4.93 & 2.10 & \\
\hline \multicolumn{5}{|l|}{ Parental Education } \\
\hline Mother Education & 9503 & 15.24 & 3.00 & \\
\hline Father Education & 9127 & 15.40 & 3.24 & \\
\hline Biological Parent Drinking & 8456 & & & $27.6 \%$ \\
\hline \multicolumn{5}{|l|}{ Ethnicity } \\
\hline White & 4881 & & & $50.1 \%$ \\
\hline Black & 1873 & & & $19.2 \%$ \\
\hline Asian & 1615 & & & $16.6 \%$ \\
\hline Hispanic & 594 & & & $6.1 \%$ \\
\hline \multicolumn{5}{|l|}{ Gender } \\
\hline Male & 3780 & & & $38.5 \%$ \\
\hline Female & 6040 & & & $61.5 \%$ \\
\hline
\end{tabular}

Table 1 demonstrates a mean of higher than 15 for both parents' education, meaning that the average parent in our sample attended some college or completed college.

\section{Parenting Style and Substance Use}

Table 2 demonstrates the relationships between the parenting styles and alcohol use when biological parent drinking problem, religiosity, mother education, father education, ethnicity, and gender were controlled. The relationship between parenting style and nicotine use is also demonstrated with religiosity, mother education, father education, ethnicity, and gender controlled. Linear regression analysis was conducted between parenting style, moderators, and alcohol use. There was a significant, weak, and negative relationship between $A G$ and alcohol use $(B=-0.033, p=0.006)$. Ordinal regression analysis was conducted between parenting style, moderators, and nicotine use. Results revealed a significant, weak, negative relationship between AG and nicotine use $(B=-0.037, p<0.001)$.

\begin{tabular}{llll}
\hline Variable & $\boldsymbol{B}$ & $\boldsymbol{S E} \boldsymbol{B}$ & $\boldsymbol{P}$-value \\
\hline $\begin{array}{l}\text { Alcohol Use* } \\
\quad \text { Parenting Style: Involvement }\end{array}$ & 0.012 & 0.014 & 0.385 \\
$\quad \begin{array}{l}\text { Parenting Style: Autonomy } \\
\quad \text { Granting }\end{array}$ & -0.033 & 0.012 & $0.006^{* *}$ \\
$\begin{array}{l}\text { Nicotine Use } \\
\quad \text { Parenting Style: Involvement }\end{array}$ & -0.012 & 0.014 & 0.381 \\
$\quad \begin{array}{l}\text { Parenting Style: Autonomy } \\
\text { Granting }\end{array}$ & -0.066 & 0.013 & $<0.001^{* *}$ \\
\hline Table 2. Regressions of Parenting Style on Past Month Alcohol and Nicotine Use. *Controlled for biological parent drinking problem. **p $<0.05$.
\end{tabular}




\section{Moderators}

Linear regression analysis was used to analyze the relationship between moderators (gender, ethnicity, religiosity, parental education), biological parent drinking problems, and alcohol use. Religiosity, Black race, Asian race, and female gender were negatively associated with alcohol use ( $B$ range: $-0.051--0.667, p=<0.001)$, while biological parent drinking problem and father education was positively associated with alcohol use $(B$ range: $0.144-0.023$, $p$ range: $<0.001-0.015)$. Mother education was found to be positively associated with alcohol use under Autonomy Granting. Ordinal regression analysis was conducted to analyze the relationship between the moderators and nicotine use. There was a moderate relationship between all moderators, except, mother education level, father education level, and Hispanic/Latino ethnicity and nicotine use $(p$ range: $<0.001-0.005)$. Religiosity demonstrated a negative association with nicotine use ( $B$ range: $-0.10--0.107, p<0.001)$. Being male, and white race were positively associated with nicotine use ( $B$ range: $0.419-0.799, p<0.001)$.

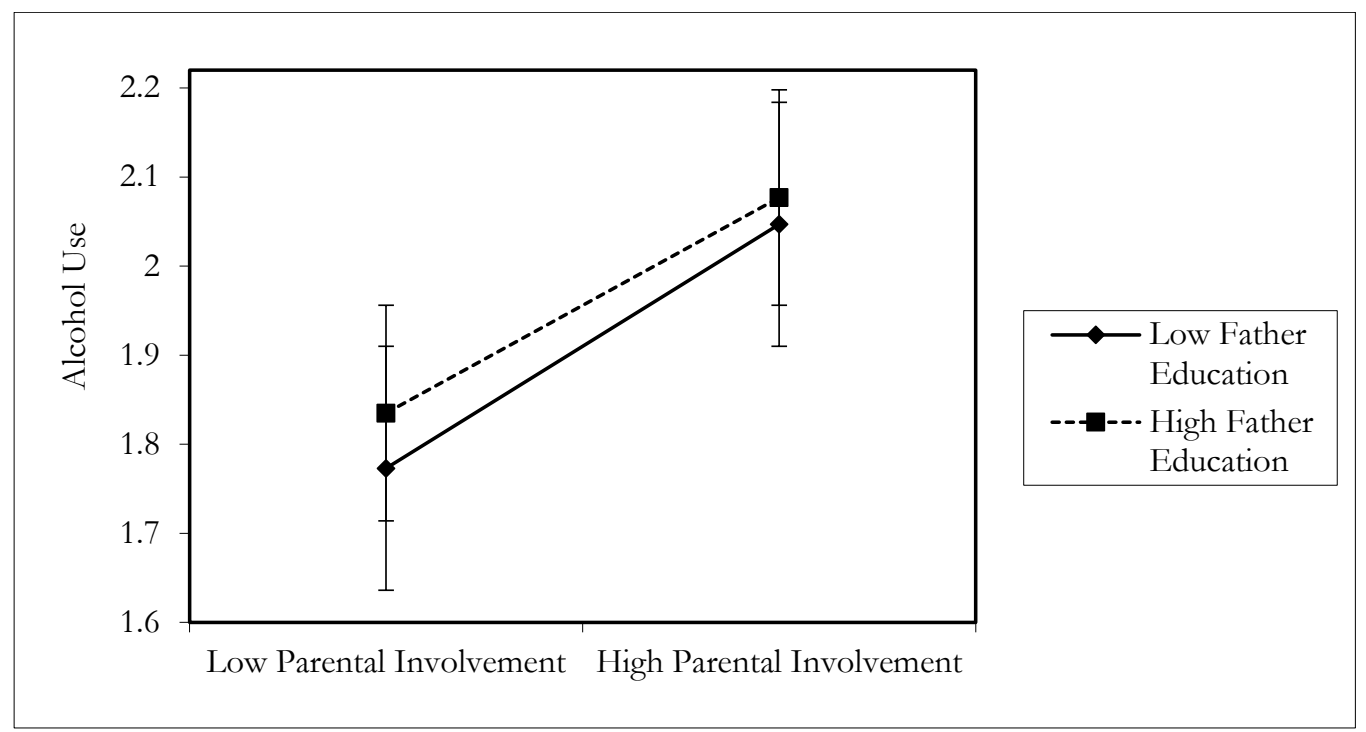

Figure 1. Line Graph Demonstrating Father Education Moderation of Parental Involvement and Alcohol Use \pm SEM.

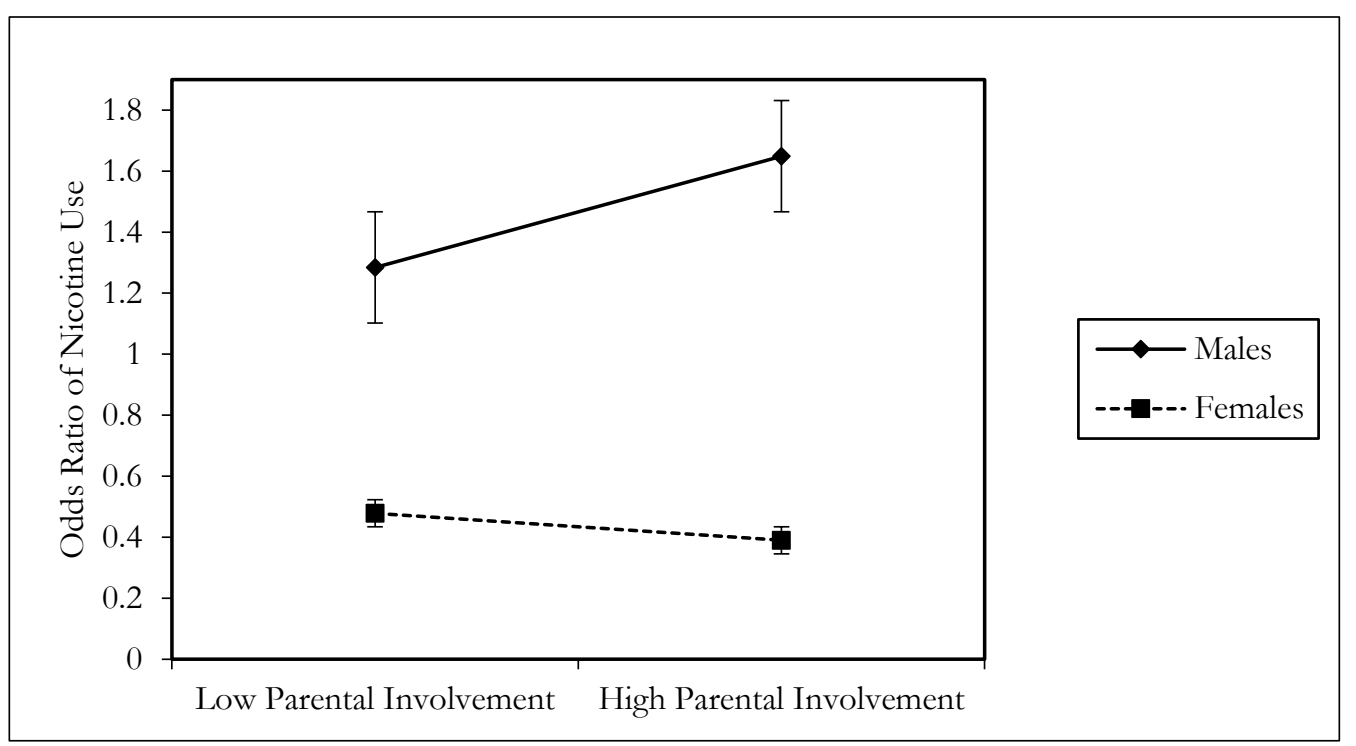

Figure 2. Line Graph Demonstrating Gender Moderation of Parental Involvement and Nicotine Use \pm SEM.

The main effect of father's education on alcohol use was a significant, weak, positive relationship $(B=0.023, p$ range: $0.015-$ 0.018). As demonstrated by Figure 1, father education level was observed to have a borderline significant, weak, negative moderation between PI and alcohol use $(B=-0.008, p=0.056)$, meaning that under high parental involvement there is greater alcohol use across both parent's education levels. Under high father education, there is greater alcohol use across PI levels. The difference of drinking levels is greater between education levels under low PI when compared to high PI. Meaning that, there is 
less drinking among students who reported low PI and low father education than those that reported low PI and high father education.

The main effect of gender on nicotine use was observed to have a significant, moderate, positive relationship $(B=0.440, p=<$ 0.001). We found no significant moderators of AG and alcohol use, as well as AG and nicotine use. Only gender demonstrated moderation between PI and nicotine use; a significant, weak, negative moderation $(B=-0.094, p=0.001)$, meaning that Males with higher levels of PI were associated with higher levels of nicotine use, while Females with higher levels of PI were associated with lower levels of nicotine use. Overall, Females had lower levels of nicotine use than Males. Gender moderation of PI and nicotine use is shown by Figure 2.

\section{DISCUSSION}

This study examined the relationship between parenting styles and alcohol and nicotine use outcomes within a large and diverse group of university students. In addition, the current study sought to test potential moderating factors of the relationship between parenting styles and alcohol and nicotine use such as religiosity, ethnicity, gender, and parent education level. We found that there was no significant relationship between PI and alcohol and nicotine use. AG demonstrated a negative association with alcohol and nicotine use, however it was a weak association. Based on previous research parenting style may play a bigger role in adolescents' substance use prior to college; however, the influence may decrease once they go to university. This could be due to the emerging adult no longer living with their parents and thus being more susceptible to peer influences. The finding is consistent with previous findings demonstrating that parenting style may be less important in the development of early adult substance use. ${ }^{20}$ These results contradict the study by Hartman and colleagues, but this may not be surprising due to the differences between our studies. ${ }^{7}$ Hartman and colleagues focused on the effect of the parenting styles of each individual parent on the university student, while our study focused on the combined effect of parenting styles of both parents. This difference is important to note because other factors, such as gender of the parent, could influence the results.

All moderators (religiosity, ethnicity, gender, and mother/father education level) were found to be significant predictors of alcohol use. Other studies reveal similar results. For example, El Ansari and colleagues found religiosity to have a positive association with the lack of drug use among university students. ${ }^{9}$ Similarly, our analysis demonstrated a negative association of religiosity and alcohol use, the same results were found concerning the relationship between religiosity and nicotine use. However, the El Ansari and colleagues' study was focused more on illicit drug use, whereas our study was aimed towards alcohol and nicotine use. Alcohol, nicotine, and other illicit drugs are all considered psychoactive substances. The abuse of such substances lead to drug dependence. ${ }^{21}$ Therefore, the findings may support each other. We found a significant, moderate, negative relationship between gender and alcohol and nicotine use, indicating that females smoke and drink less than males. This pattern is consistent with past findings in which males were found to use drugs twice as often as females. ${ }^{9}$

Analysis of moderation demonstrated father education level to have a borderline significant, but weak, negative moderating effect between PI and alcohol use. Students whose fathers have high education level drink more than students with low father education level across both PI levels. However, difference between drinking levels was found to be greater between education levels under low PI compared to high PI. Droomers and colleagues also assessed the association between occupational level of the father and high alcohol consumption in adolescents ages 11 to $21 .^{22}$ In addition, the study looked to find factors that explained the association between father occupation level and adolescent drinking. In contrast to our findings, Droomers and colleagues, found that low father occupation, and low parental attachment has a significant increase in the odds of high alcohol consumption. Parental Attachment was assessed in the Droomers and colleagues article using the Inventory of Parent and Peer Attachment (IPPA) scale developed by Armsden and Greenburg, 1987.23 The scale measures of the IPPA had a degree of similarity with our scales as they evaluated Alienation, Communication, and Trust. Droomers and colleagues' finding is still relevant because it assesses communication and awareness of the child's emotions, which is also evaluated in PI. Humensky carried out a study to examine the relationship between adolescent socioeconomic status (measured by parental education and household income) and adolescent substance use (alcohol and illicit drugs). ${ }^{24}$ Findings demonstrated a higher association between adolescent binge drinking, marijuana, and cocaine use when adolescent socioeconomic status was high. ${ }^{24}$ Since higher levels of education are associated with high socioeconomic status, ${ }^{11}$ it could explain the consistently high alcohol use among participants of higher father education level, compared to participants of lower father education level, in both low and high PI. Nonetheless, it is perplexing to find that low father education and low PI are associated with less alcohol usage among university students.

Gender was found to demonstrate a significant, weak, negative moderation between PI and nicotine use. Females tended to have less nicotine use when under both low and high PI. Overall, male participants who reported high PI seemed to have significantly greater nicotine use compared to females who reported high PI. The higher nicotine use among males could be explained by a study conducted by Carroll and colleagues. ${ }^{25}$ This study examined smoking in college students and the results revealed that males were more likely to be current smokers than females. In addition, one of the aspects of PI is parent-adolescent communication and discussion. ${ }^{26}$ A previous study with Bahamian adolescents has found that problematic parent-adolescent communication demonstrated a consistent increase in substance use behaviors, sexual risk behaviors, and delinquent behaviors. ${ }^{26}$ Since males demonstrated greater nicotine use than females who reported high PI, it can be speculated that gender differences play a role in the quality of parent-adolescent communication. This can be explained by a study conducted on Italian adolescents which found 
that female adolescents reported more open communication with their parents, especially with their mothers, than male adolescents. ${ }^{27}$ The increase in problematic parent-adolescent communication could explain the higher nicotine use in males who reported high PI.

\section{CONCLUSIONS}

The data used in this study is from a large survey that focuses on understanding the genetic and environmental conditions that lead to substance use in university students. This, along with the representative, diverse sample and appropriate age group, are strengths of this study. An important limitation is that this study examined students around the time of matriculation into university. Entering university is a time in which students have obtained a new degree of freedom and have the opportunity to experiment with risk behaviors. By following the participants throughout their time in university we would have been able to analyze data from their alcohol and nicotine habits, rather than just obtaining data that may have been from experimenting and trying to gain new experiences. Another important limitation is that this study did not have the data to control nicotine relationships for parental nicotine use, as done with alcohol relationships.

Future directions of this study could be to assess whether or not other factors influence alcohol and nicotine use such as home environment, conflict, extracurricular activities, and peer usage. Other studies could aim to obtain parental report of parenting styles and use the data, in addition to the participant's report on parenting style, to determine the relationship with alcohol and nicotine use among the participants. In addition, the study could examine alcohol and nicotine use over time. Examining these factors is important in that it could help better inform the population regarding the outlying factors that associate with alcohol and nicotine use. It could also help improve treatment and intervention programs.

\section{ACKNOWLEDGEMENTS}

Spit for Science: The VCU Student Survey has been supported by Virginia Commonwealth University, P20 AA107828, R37AA011408, K02AA018755, and P50 AA022537 from the National Institute on Alcohol Abuse and Alcoholism, and UL1RR031990 from the National Center for Research Resources and National Institute of Health Roadmap for Medical Research. We thank the VCU students for making this study a success, as well as the many VCU faculty, students, and staff who contributed to the design and implementation of the project. We are grateful to Kenneth S. Kendler for his support of this Spit for Science project.

\section{REFERENCES}

1. Bjartveit, K., and Tverdal, A. (2005). Health consequences of smoking 1-4 cigarettes per day. Tob Control, 14(5), 315-320. http://doi.org/10.1136/tc.2005.011932

2. Mathiesen, E. F., Nome, S., Eisemann, M., and Richter, J. (2012). Drinking patterns, psychological distress and quality of life in a Norwegian general population-based sample. Qual Life Res, 21(9), 1527-1536. http://doi.org/10.1007/s11136-011-0080-8

3. Perkins, H. W. (2002). Surveying the damage: a review of research on consequences of alcohol misuse in college populations. $J$ Stud Alcohol, Supplement(14), 91-100. http://dx.doi.org//10.15288/jsas.2002.s14.91

4. Hingson, R. W., Zha, W., and Weitzman, E. R. (2009). Magnitude of and trends in alcohol-related mortality and morbidity among U.S. college students ages 18-24, 1998-2005. J Stud Alcohol Drugs Suppl, (16), 12-20.

5. Hernandez, L., Rodriguez, A. M., and Spirito, A. (2015). Brief Family-Based Intervention for Substance Abusing Adolescents. Child Adol Psych Cl, 24(3), 585-599. http://doi.org/10.1016/j.chc.2015.02.010

6. Fromme, K., Corbin, W. R., and Kruse, M. I. (2008). Behavioral risks during the transition from high school to college. Dev Psychol, 44(5), 1497-1504. http://doi.org/10.1037/a0012614

7. Hartman, J. D., Patock-Peckham, J. A., Corbin, W. R., Gates, J. R., Leeman, R. F., Luk, J. W., and King, K. M. (2015). Direct and indirect links between parenting styles, self-concealment (secrets), impaired control over drinking and alcohol-related outcomes. Addict Behav, 40, 102-108. http://doi.org/10.1016/j.addbeh.2014.08.009

8. Patock-Peckham, J. A., Cheong, J., Balhorn, M. E., and Nagoshi, C. T. (2001). A social learning perspective: a model of parenting styles, self-regulation, perceived drinking control, and alcohol use and problems. Alcohol Clin Exp Res, 25(9), 12841292.

9. El Ansari, W., Vallentin-Holbech, L., and Stock, C. (2015). Predictors of illicit drug use among university students in Northern Ireland, Wales and England. Global J of Healt Sci, 7(4), 18-29. http://doi.org/10.5539/gjhs.v7n4p18

10. Bachman, J.G. (2002). The Decline of Substance Use in Young Adulthood: Changes in Social Activities, Roles and Beliefs. Mahwah, N.J: Psychol Press.

11. Davis-Kean, P. E. (2005). The Influence of Parent Education and Family Income on Child Achievement: The Indirect Role of Parental Expectations and the Home Environment. J Fam Psychol, 19(2), 294-304. http://doi.org/10.1037/0893-3200.19.2.294

12. He, J., Assanangkornchai, S., Cai, L., and McNeil, E. (2016). Disparities in drinking patterns and risks among ethnic majority and minority groups in China: The roles of acculturation, religion, family and friends. Drug Alcohol Depen, 159, $198-206$. http://doi.org/10.1016/j.drugalcdep.2015.12.028 
13. Lamborn, S. D., Mounts, N. S., Steinberg, L., and Dornbusch, S. M. (1991). Patterns of Competence and Adjustment among Adolescents from Authoritative, Authoritarian, Indulgent, and Neglectful Families. Child Dev, 62(5), 1049. http://doi.org/10.1111/1467-8624.ep9112161645

14. Steinberg, L., Lamborn, S. D., Dornbusch, S. M., and Darling, N. (1992). Impact of Parenting Practices on Adolescent Achievement: Authoritative Parenting, School Involvement, and Encouragement to Succeed. Child Dev, 63(5), $1266-1281$. http://doi.org/10.2307/1131532

15. Dick, D., Nasim, A., Edwards, A. C., Salvatore, J., Cho, S. B., Adkins, A., ... and Kendler, K. S. (2014). Spit for Science: launching a longitudinal study of genetic and environmental influences on substance use and emotional health at a large US university. Behav Psych Genet, 5, 47. http://doi.org/10.3389/fgene.2014.00047

16. Harris, P. A., Taylor, R., Thielke, R., Payne, J., Gonzales, N., and Conde, J. G. (2009). Research electronic data capture (REDCap) - A metadata-driven methodology and workflow process for providing translational research informatics support. $J$ Biomed Inform, 42(2), 377-381. http://dx.doi.org/10.1016/j.jbi.2008.08.010

17. Bush, K., Kivlahan, D. R., McDonell, M. B., Fihn, S.D., \& Bradley, K. A. (1998). The AUDIT alcohol consumption questions (AUDIT-C): An effective brief screening test for problem drinking. Arch Intern Med, 158(16), 1789-1795. http://dx.doi.org/10.1001/archinte.158.16.1789

18. Sher, K. J., and Rutledge, P. C. (2007). Heavy drinking across the transition to college: Predicting first-semester heavy drinking from precollege variables. Addict Behav, 32(4), 819-835. http://dx.doi.org/10.1016/j.addbeh.2006.06.024

19. Kendler, K. S., Gardner, C. O., and Prescott, C. A. (1997). Religion, psychopathology, and substance use and abuse; a multimeasure, genetic-epidemiologic study. Am J Pysch, 154(3), 322-329. http://dx.doi.org/10.1176/ajp.154.3.322

20. Berge, J., Sundell, K., Öjehagen, A., and Håkansson, A. (2016). Role of parenting styles in adolescent substance use: results from a Swedish longitudinal cohort study. BMJ Open, 6(1), e008979. http://doi.org/10.1136/bmjopen-2015-008979

21. Parrott, A. (2004). Understanding Drugs and Behaviour. Chichester, West Sussex, Eng: John Wiley and Sons, Inc.

22. Droomers, M., Schrijvers, C., Casswell, S., and Mackenbach, J. (2003). Occupational level of the father and alcohol consumption during adolescence; patterns and predictors. J Epidemiol Commun H, 57(9), 704-710. http://doi.org/10.1136/jech.57.9.704

23. Armsden, G.C., Greenberg, M. T. (1987). The inventory of parent and peer attachment: Individual differences and their relationship to psychological well-being in adolescence. J Youth Adolesc, 16(5), 427-454. http://doi.org/10.1007/BF02202939

24. Humensky, J. L. (2010). Are adolescents with high socioeconomic status more likely to engage in alcohol and illicit drug use in early adulthood? Subst Abuse Treat Pr, 5, 19. http://doi.org/10.1186/1747-597X-5-19

25. Carroll, S. L., Lee, R. E., Kaur, H., Harris, K. J., Strother, M. L., and Huang, T. T.-K. (2006). Smoking, Weight Loss Intention and Obesity-Promoting Behaviors in College Students. J Am Coll Nutr, 25(4), 348-353. http://doi.org/10.1080/07315724.2006.10719545

26. Wang, B., Stanton, B., Li, X., Cottrell, L., Deveaux, L., and Kaljee, L. (2013). The influence of parental monitoring and parent-adolescent communication on Bahamian adolescent risk involvement: A three-year longitudinal examination. Soc $S_{c i}$ Med, 97, 161-169. http://doi.org/10.1016/j.socscimed.2013.08.013

27. Rosnati, R., Iafrate, R., and Scabini, E. (2007). Parent-adolescent communication in foster, inter-country adoptive, and biological Italian families: Gender and generational differences. Int J Psychol, 42(1), 36-45.

http://doi.org/10.1080/00207590500412128

\section{ABOUT THE STUDENT AUTHOR}

Zackaria Niazi is currently pursuing a B.S. in Biology at Virginia Commonwealth University (VCU). He worked with Spit for Science as an Undergraduate Research Assistant for one year. Currently, he is an Undergraduate Research Assistant for the Service Experiences and Alcohol Preference study at the Virginia Institute for Psychiatric and Behavioral Genetics. In addition to being involved in research, Zackaria is the Vice President of the Health Disparities Organization at VCU.

\section{PRESS SUMMARY}

Overall, both alcohol and nicotine use among university students have demonstrated a compromising effect on physical health, mental health, and/or social relationships. To help improve future intervention methods, this study sought to find the relationship between parenting styles and alcohol/nicotine use of university students. In addition, it sought to find moderators of the relationship. The results indicate that parenting styles may play little role in the development of alcohol and nicotine use among university students. Additionally, this study found that father education demonstrated borderline significant moderation between Parental Involvement and alcohol use of university students. Gender was found to demonstrate significant moderation between Parental Involvement and nicotine use of university students. No moderators were found between Autonomy Granting and alcohol use, nor Autonomy Granting and nicotine use. 


\title{
Matricial Representations of Certain Finitely Presented Groups Generated by Order-2 Generators and Their Applications
}

\author{
Ryan Golden*, Ilwoo Cho \\ Department of Mathematics, Saint Ambrose University, Davenport, IA \\ Students: goldenryanm@sau.edu* \\ Mentor: choilwoo@sau.edu
}

\begin{abstract}
In this paper, we study matricial representations of certain finitely presented groups $\Gamma_{N}^{2}$ with $N$-generators of order-2. As an application, we consider a group algebra $\mathcal{A}_{2}$ of $\Gamma_{2}^{2}$, under our representations. Specifically, we characterize the inverses $g^{-1}$ of all group elements $g$ in $\Gamma_{2}^{2}$, in terms of matrices in the group algebra $\mathcal{A}_{2}$. From the study of this characterization, we realize there are close relations between the trace of the radial operator of $\mathcal{A}_{2}$, and the Lucas numbers appearing in the Lucas triangle.
\end{abstract}

\section{KEYWORDS}

Matricial Representation; Group Presentation; Group Algebras; Lucas Numbers; Lucas Triangle; Finitely Presented Group; Group Relations; Free Probability

\section{INTRODUCTION}

In this paper, we consider certain finite-dimensional representations $\left(\mathbb{C}^{n}, \alpha^{n}\right)$ of a finitely presented group:

$$
\Gamma_{N}^{2} \stackrel{\text { def }}{=}\left\langle\left\{x_{1}, \ldots, x_{N}\right\},\left\{x_{1}^{2}=\ldots=x_{N}^{2}\right\}\right\rangle
$$

Equation 1.

where the generators $x_{1}, \ldots, x_{N}$ are indeterminants. Precisely, the algebraic structure $\Gamma_{N}^{2}$ is the group generated by $N$ generators $x_{1}, \ldots, x_{N}$ equipped with a noncommutative binary operation (Denoted by adjacency), satisfying

$$
x_{1}^{2}=x_{2}^{2}=\ldots=x_{N}^{2}=e_{N},
$$

Equation 2.

where $e_{N}$ is the group-identity of $\Gamma_{N}^{2}$. One may understand a group $\Gamma_{N}^{2}$ as the quotient group,

$$
\Gamma_{N}^{2} \stackrel{\text { Group }}{=} \mathcal{F}_{N} /\left\{g_{j}^{2}=e: g_{j} \text { are generators of } \mathcal{F}_{N}\right\},
$$

Equation 3.

where $\mathcal{F}_{N}$ is the noncommutative (non-reduced) free group $\left\langle\left\{g_{j}\right\}_{j=1}^{N}\right\rangle$ with $N$ generators $g_{1}, \ldots, g_{N}$, where $e$ is the group-

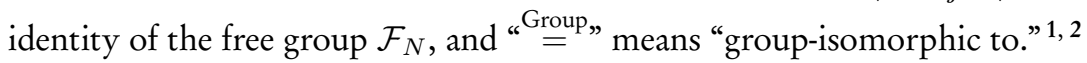

By construction, all elements $g$ of the group $\Gamma_{N}^{2}$ are of the form,

$$
g=x_{i_{1}}^{k_{1}} x_{i_{2}}^{k_{2}} \ldots x_{i_{n}}^{k_{n}}, \text { for some } i_{1}, \ldots, i_{n} \in\{1, \ldots, N\} \text {, and } k_{1}, \ldots, k_{n} \in \mathbb{Z},
$$

Equation 4.

as non-reduced words in $\left\{x_{1}, \ldots, x_{N}\right\}$, where $x_{j}^{-1}$ means the group-inverse of $x_{j}$, and hence, $x_{j}^{-k}$ means $\left(x_{j}^{-1}\right)^{k}$, for all $k \in \mathbb{N}$, for all $j=1, \ldots N$. In this paper, for an arbitrarily fixed $N \in \mathbb{N}$, we establish and study certain $n$-dimensional Hilbert-space representations $\left(\mathbb{C}^{n}, \alpha^{n}\right)$ of the groups $\Gamma_{N}^{2}$, for all $n \in \mathbb{N} \backslash\{1\}$. Under our representations, each element $g$ of $\Gamma_{N}^{2}$ is understood as a matrix $A_{g}$ acting on $\mathbb{C}^{n}$, for $n \in \mathbb{N}$. Moreover, if $g=x_{i_{1}}^{k_{1}} \ldots x_{i_{l}}^{k_{l}}$ as in Equation 4 (as a non-reduced word in $\Gamma_{N}^{2}$ ), then there exists corresponding matrices $A_{i_{1}}, \ldots, A_{i_{l}}$, such that $A_{g}=A_{i_{1}}^{k_{1}} \ldots A_{i_{l}}^{k_{l}} \in M_{n}(\mathbb{C}$ ) (as a reduced word in $M_{n}(\mathbb{C})$ ), where $M_{n}(\mathbb{C})$ is the matricial algebra consisting of all $(n \times n)$-matrices, for $n \in \mathbb{N}$. 


\section{Motivation}

Here we justify our interest in the groups $\Gamma_{N}^{2}$, for $N \in \mathbb{N}$.

Free probability is a branch of operator algebra. By considering free-distributional data, one can establish operator-valued noncommutative probability theory on (topological or pure-algebraic) algebras as in classical probability theory. ${ }^{3,4}$ Also, such free-probabilistic data allow structure theorems for given algebras under free product, determined by given linear functionals. Here, the independence of classical probability is replaced by freeness. There are two approaches in free probability theory: Voiculescu's original analytic approach, ${ }^{4}$ and Speicher's combinatorial approach. ${ }^{3}$

The author, Cho, has considered connections between operator algebra theory and Hecke-algebraic number theory via free probability, to provide tools for studying number-theoretic results with operator-algebraic techniques, and vice versa, by establishing certain representational and operator-theoretic models from combinatorial free probability settings. ${ }^{3}$

In a series of research papers, Cho considered the free-probabilistic representations of the Hecke Algebras $\mathcal{H}\left(G_{p}\right)$ generated by the generalized linear $\left((2 \times 2)\right.$-matricial) groups, $G_{p}=G L_{2}\left(\mathbb{Q}_{p}\right)$, over the $p$-adic number field $\mathbb{Q}_{p}$ for prime $p .{ }^{1,2,5}$

Cho and Gillespie established free probability models of certain subalgebras $\mathcal{H}_{Y_{p}}$ of the Hecke algebras $\mathcal{H}\left(G_{p}\right)$ by defining suitable linear functionals on $\mathcal{H}\left(G_{p}\right)$. In particular, they constructed free-probabilistic structures preserving numbertheoretic data from $\mathcal{H}_{Y_{p}} \cdot{ }^{5}$

Cho extended certain free-probabilistic models of $\mathcal{H}_{Y_{p}}$ to those of $\mathcal{H}\left(G_{p}\right)$ fully, for all primes $p .{ }^{2,6}$ On such models, the $C^{*}$-algebras $\mathbb{H}\left(G_{p}\right)$ were constructed by realizing elements of $\mathcal{H}\left(G_{p}\right)$ as operators under free-probabilistic representations of $\mathcal{H}\left(G_{p}\right)$, and the operator-theoretic properties of generating operators of $\mathbb{H}\left(G_{p}\right)$ were studied there.

By studying certain types of partial isometries of $\mathbb{H}\left(G_{p}\right)$, Cho obtained the embedded non-abelian multiplicative groups $G_{N}$ of $\mathbb{H}\left(G_{p}\right)$ generated by $N$-many partial isometries. ${ }^{1}$ In particular, it was shown that

$$
G_{N} \stackrel{\text { Group }}{=} \Gamma_{N}^{2}, \text { for all } N \in \mathbb{N},
$$

Equation 5.

where $\Gamma_{N}^{2}$ is in the sense of Equation 1, satisfying Equation 3. To study detailed algebraic properties of $G_{N}$ in $\mathcal{H}\left(G_{p}\right)$, and to investigate operator-algebraic properties of $C^{*}\left(G_{N}\right)$ in $\mathbb{H}\left(G_{p}\right)$, he used the isomorphic group $\Gamma_{N}^{2}$ of Equation 1, and the corresponding group $C^{*}$-algebra: $C^{*}\left(\Gamma_{N}^{2}\right)$.

In this paper, we study the groups $\Gamma_{N}^{2}$ of Equation 1 pure-algebraically (independent from our sources). ${ }^{1-3}$

\section{Overview}

In this paper, we concentrate on studying the groups $\Gamma_{N}^{2}$ of Equation 1 independently. The main purpose is to establish suitable Hilbert-space representations of $\Gamma_{N}^{2}$ other than those established in our sources. ${ }^{1}$ Fundamentally, we construct finite-dimensional Hilbert-space representations $\left(\mathbb{C}^{n}, \alpha^{n}\right)$ of $\Gamma_{N}^{2}$, for all $n \in \mathbb{N} \backslash\{1\}$.

Under our representation $\left(\mathbb{C}^{n}, \alpha^{n}\right)$ for $n \in \mathbb{N}$, each group element $g \in \Gamma_{N}^{2}$ is understood as a matrix $A_{g}$ in the matricial algebra $M_{n}(\mathbb{C})$. To study an algebraic object $g \in \Gamma_{N}^{2}$, we will investigate functional properties of the corresponding matrices $A_{g} \in M_{n}(\mathbb{C})$, for $n \in \mathbb{N}$.

As application, we consider a group algebra $\mathcal{A}_{2}$ of $\Gamma_{2}^{2}$, under our representations. Specifically, we characterize the inverses $g^{-1}$ of all group elements $g$ in $\Gamma_{2}^{2}$, in terms of matrices in the group algebra $\mathcal{A}_{2}$ generated by $\Gamma_{2}^{2}$.

From the study of this characterization, we show that there are close relations between the trace of the powers $T^{n}$ of the radial operator $T$ of $\mathcal{A}_{2}$, and the Lucas numbers in the Lucas triangle. 


\section{FINITE-DIMENSIONAL REPRESENTATIONS OF $\Gamma_{N}^{2}$}

In this section, we establish finite-dimensional Hilbert-space representations of the finitely presented groups $\Gamma_{N}^{2}$ with $N$ generators of order-2 in the sense of Equation 1: $\Gamma_{N}^{2}=\left\langle\left\{x_{j}\right\}_{j=1}^{N},\left\{x_{j}^{2}=e_{N}\right\}_{j=1}^{N}\right\rangle$, where $x_{1}, \ldots, x_{j}$ are noncommutative indeterminants (as generators), and $e_{N}$ is the group identity of $\Gamma_{N}^{2}$. Fix $N \in \mathbb{N}$ throughout this section and fix the corresponding group $\Gamma_{N}^{2}$. Denote by $I_{N}$ the identity matrix in $M_{N}(\mathbb{C})$

\section{A Matrix Group $\mathfrak{M}_{2}^{2}(N)$}

Let $M_{2}(\mathbb{C})$ be the $(2 \times 2)$-matricial algebra, and let $\mathbb{C}^{\times}=\mathbb{C} \backslash\{0\}$. Denote by $\mathbb{C} \times \mathbb{C}^{\times}$the Cartesian product of $\mathbb{C}^{\text {and }} \mathbb{C}^{\times}$. Define a function

$$
A_{2}: \mathbb{C} \times \mathbb{C}^{\times} \rightarrow M_{2}(\mathbb{C}) \text { by } A_{2}(a, b)=\left(\begin{array}{cc}
a & b \\
\frac{1-a^{2}}{b} & -a
\end{array}\right)
$$

Equation 6.

for all $(a, b) \in \mathbb{C} \times \mathbb{C}^{\times}$.

Lemma 1. Let $A$ be the map in the sense of Equation 6. Then

$$
\left(A_{2}(a, b)\right)^{2}=I_{2}
$$

Equation 7.

for all $(a, b) \in \mathbb{C} \times \mathbb{C}^{\times}$.

Proof. The proof is straightforward computation.

It is also easy to verify that:

$$
\begin{aligned}
& A_{2}\left(a_{1}, b_{1}\right) A_{2}\left(a_{2}, b_{2}\right)=\left(\begin{array}{cc}
\frac{a_{1} a_{2} b_{2}+b_{1}-b_{1} a_{2}^{2}}{b_{2}} & a_{1} b_{2}-b_{1} a_{2} \\
\frac{a_{2} b_{2}-a_{2} b_{2} a_{1}^{2}-a_{1} b_{1}+a_{1} b_{1} a_{2}^{2}}{b_{1} b_{2}} & \frac{b_{2}-b_{2} a_{1}^{2}+a_{1} a_{2} b_{1}}{b_{1}}
\end{array}\right) \\
& A_{2}\left(a_{2}, b_{2}\right) A_{2}\left(a_{1}, b_{1}\right)=\left(\begin{array}{cc}
\frac{b_{2}-b_{2} a_{1}^{2}+a_{1} a_{2} b_{1}}{b_{1}} & b_{1} a_{2}-a_{1} b_{2} \\
\frac{-a_{2} b_{2}+a_{2} b_{2} a_{1}^{2}+a_{1} b_{1}-a_{1} b_{1} a_{2}^{2}}{b_{1} b_{2}} & \frac{a_{1} a_{2} b_{2}+b_{1}-b_{1} a_{2}^{2}}{b_{2}}
\end{array}\right)
\end{aligned}
$$

Equation 8.

and hence,

$$
A_{2}\left(a_{1}, b_{1}\right) A_{2}\left(a_{2}, b_{2}\right) \neq A_{2}\left(a_{2}, b_{2}\right) A_{2}\left(a_{1}, b_{1}\right)
$$

Equation 9.

in general, for $\left(a_{k}, b_{k}\right) \in \mathbb{C} \times \mathbb{C}^{\times}$and $k=1$, 2. In particular, whenever $\left(a_{k}, b_{k}\right) \in \mathbb{C}^{\times} \times \mathbb{C}^{\times}$, for $k=1,2$, and the pairs $\left(a_{1}, b_{1}\right)$ and $\left(a_{2}, b_{2}\right)$ are distinct in $\mathbb{C}^{\times} \times \mathbb{C}^{\times}$, the above noncommutativity Equation $\mathbf{9}$ always holds. Therefore, the image, $\left\{A_{2}(a, b):(a, b) \in \mathbb{C}^{\times} \times \mathbb{C}^{\times}\right\}$, forms a noncommutative family in $M_{2}(\mathbb{C})$, by Equation 9.

Now, take distinct pairs,

$$
\left(a_{k}, b_{k}\right) \in \mathbb{C}^{\times} \times \mathbb{C}^{\times}, \text {for } k=1, \ldots, N .
$$

Equation 10.

One can construct the corresponding matrices,

$$
A_{2}\left(a_{k}, b_{k}\right) \in M_{2}(\mathbb{C}), \text { for } k=1, \ldots, N,
$$

Equation 11.

by Equation 6. Under the matrix multiplication on $M_{2}(\mathbb{C})$, let's construct the multiplicative subgroup $\mathfrak{M}_{2}^{2}(N)$ of $M_{2}(\mathbb{C})$ as

$$
\mathfrak{M}_{2}^{2}(N)=\left\langle\left\{A_{2}\left(a_{k}, b_{k}\right)\right\}_{k=1}^{N}\right\rangle
$$

Equation 12.

generated by $A_{2}\left(a_{k}, b_{k}\right)$, for all $k=1, \ldots, N$. Then all elements of $\mathfrak{M}_{2}^{2}(N)$ are the $(2 \times 2)$-matrices in $M_{2}(\mathbb{C})$, satisfying

$$
\left(A_{2}\left(a_{k}, b_{k}\right)\right)^{2}=I_{2} \in \mathfrak{M}_{2}^{2}(N),
$$

Equation 13.

for all $k=1, \ldots, N$, by Equation 7 . 
Definition 1. Let $\mathfrak{M}_{2}^{2}(N)$ be the multiplicative subgroup of $M_{2}(\mathbb{C})$, as defined in Equation 12. We call $\mathfrak{M}_{2}^{2}(N)$ the 2-dimensional, order-2, $N$-generator (sub-)group (of $M_{2}(\mathbb{C})$ ).

One obtains the following algebraic characterization.

Theorem 2. Let $\mathfrak{M}_{2}^{2}(N)$ be a 2-dimensional, order-2, $N$-generator group in $M_{2}(\mathbb{C})$. Then the groups $\mathfrak{M}_{2}^{2}(N)$ and the group $\Gamma_{N}^{2}$ of Equation 1 are isomorphic:

$$
\mathfrak{M}_{2}^{2}(N) \stackrel{\text { Group }}{=} \Gamma_{N}^{2}
$$

Equation 14.

Proof. Let $\mathfrak{M}_{2}^{2}(N)$ be a 2-dimensional, order-2, $N$-generator group in $M_{2}(\mathbb{C})$. Then, by Equation 13, each generator $A_{2}\left(a_{k}, b_{k}\right)$ satisfies $\left(A_{2}\left(a_{k}, b_{k}\right)\right)^{2}=I_{2}$, for all $k=1, \ldots, N$.

Since $\left(a_{k}, b_{k}\right)$ are taken from $\mathbb{C}^{\times} \times \mathbb{C}^{\times}$, the generators $\left\{A_{2}\left(a_{k}, b_{k}\right)\right\}_{k=1}^{N}$ form a noncommutative family in $M_{2}(\mathbb{C})$, by Equation 9.

Observe that there does not exist an $n$-tuple $\left(j_{1}, \ldots, j_{n}\right)$ of distinct elements $j_{1}, \ldots, j_{n}$ in $\{1, \ldots, N\}$ such that

$$
\prod_{l=1}^{n} A_{2}\left(a_{k_{j_{l}}}, b_{k_{j_{l}}}\right)=A_{2}\left(a_{k_{j_{0}}}, b_{k_{j_{0}}}\right) \text {, or } \prod_{l=1}^{n} A_{2}\left(a_{k_{j_{l}}}, b_{k_{j_{l}}}\right)=I_{2}, \quad \text { Equation } 15 .
$$

for some $j_{0} \in\{1, \ldots, N\}$, by Equations 6 and 9 . These observations show that $\mathfrak{M}_{2}^{2}(N) \stackrel{\text { Group }}{=} \mathcal{F}_{N} / \mathcal{R}_{N}$, where $\mathcal{F}_{N}$ is the noncommutative free group $\left\langle\left\{g_{j}\right\}_{j=1}^{N}\right\rangle$ with $N$-generators $g_{1}, \ldots, g_{N}$, and $\mathcal{R}_{N}$ is the relator set, $\mathcal{R}_{N}=\left\{g_{j}^{2}=e\right\}_{j=1}^{N}$.

Recall that the group $\Gamma_{N}^{2}$ of Equation 1 is group-isomorphic to the quotient group $\mathcal{F}_{N} / \mathcal{R}_{N}$, by Equation 3. Therefore, one has that

$$
\mathfrak{M}_{2}^{2}(N) \stackrel{\text { Group }}{=} \mathcal{F}_{N} / \mathcal{R}_{N} \stackrel{\text { Group }}{=} \Gamma_{N}^{2} .
$$

Equation 16.

The 2-dimensional, order-2, $N$-generator group $\mathfrak{M}_{2}^{2}(N)$ is group isomorphic to the group $\Gamma_{N}^{2}$ of Equation 1 .

The groups $\mathfrak{M}_{2}^{2}(N)$ and $\Gamma_{N}^{2}$ are isomorphic to each other. Equivalently, there exists a group isomorphism $\alpha^{2}: \Gamma_{N}^{2} \rightarrow$ $\mathfrak{M}_{2}^{2}(N)$, satisfying

$$
\alpha^{2}\left(x_{j}\right)=A_{2}\left(a_{j}, b_{j}\right), \text { for all } j=1, \ldots, N .
$$

Equation 17.

The above structure theorem provides a 2-dimensional Hilbert-space representation $\left(\mathbb{C}^{2}, \alpha^{2}\right)$ of $\Gamma_{N}^{2}$.

Theorem 3. There exists a 2-dimensional Hilbert-space representation $\left(\mathbb{C}^{2}, \alpha^{2}\right)$ of $\Gamma_{N}^{2}$. Additionally, $\alpha^{2}(g)$ acting on $\mathbb{C}^{2}$ are in the sense of Equation 17, for all $g \in \Gamma_{N}^{2}$.

Proof. Since $\alpha^{2}$ of Equation 17 is a generator-preserving group-isomorphism from $\Gamma_{N}^{2}$ to $\mathfrak{M}_{2}^{2}(N)$, it satisfies

$$
\begin{gathered}
\alpha^{2}\left(g_{1} g_{2}\right)=\alpha^{2}\left(g_{1}\right) \alpha^{2}\left(g_{2}\right) \in \mathfrak{M}_{2}^{2}(N) \text { for all } g_{1}, g_{2} \in \Gamma_{N}^{2} \\
\alpha^{2}\left(g^{-1}\right)=\left(\alpha^{2}(g)\right)^{-1} \in \mathfrak{M}_{2}^{2}(N) \text { for all } g \in \Gamma_{N}^{2} .
\end{gathered}
$$

Since $\mathfrak{M}_{2}^{2}(N) \subseteq M_{2}(\mathbb{C})$, the matrices $\alpha^{2}(g)$ are acting on $\mathbb{C}^{2}$, for all $g \in \Gamma_{N}^{2}$. Therefore, the pair $\left(\mathbb{C}^{2}, \alpha^{2}\right)$ forms a Hilbertspace representation of $\Gamma_{N}^{2}$. 


\section{Certain Order-2 Matrices in $M_{n}(\mathbb{C})$}

Now, let $n>2$ in $\mathbb{N}$ and consider certain types of matrices in $M_{n}(\mathbb{C})$. In the previous section, we showed that our group $\Gamma_{N}^{2}$ of Equation 1 is group isomorphic to the multiplicative subgroup $\mathfrak{M}_{2}^{2}(N)$ of Equation 12 and hence, we obtain a natural 2-dimensional representation $\left(\mathbb{C}^{2}, \alpha^{2}\right)$ of $\Gamma_{N}^{2}$. In this section, we construct a base-stone to extend the representation $\left(\mathbb{C}^{2}, \alpha^{2}\right)$ to arbitrary $n$-dimensional representations $\left(\mathbb{C}^{n}, \alpha^{n}\right)$ of $\Gamma_{N}^{2}$, for all $n \in \mathbb{N} \backslash\{1\}$. To this end, we fix the matrices $A_{2}(a, b)$, defined in Equation 6, for use as blocks of certain matrices in $M_{n}(\mathbb{C})$. For our main purpose, we always assume that $(a, b) \in \mathbb{C}_{\sim_{1}}^{\times} \times \mathbb{C}^{\times}$, whenever we have matrices $A_{2}(a, b)$ in the following text, as in Equation 10, where $\mathbb{C}_{\sim_{1}}^{\times} \stackrel{\text { def }}{=} \mathbb{C} \backslash\{0,1\}$.

First take $n=3$, and consider a matrix $A_{3}(a, b, c)$ defined to be

$$
A_{3}(a, b, c)=\left(\begin{array}{ccc}
1 & c & \frac{-b c}{1-a} \\
0 & a & b \\
0 & \frac{1-a^{2}}{b} & -a
\end{array}\right) \in M_{3}(\mathbb{C}),
$$

Equation 18.

for some $a, b, c \in \mathbb{C}^{\times}$. This matrix $A_{3}(a, b, c)$ is the block matrix,

$$
A_{3}(a, b, c)=\left(\begin{array}{cc}
(1) & \left(\begin{array}{cc}
c & \frac{-b c}{1-a}
\end{array}\right) \\
\left(\begin{array}{l}
0 \\
0
\end{array}\right) & A_{2}(a, b)
\end{array}\right) \in M_{3}(\mathbb{C}) .
$$

Equation 19.

By straightforward computation, one can obtain

$$
A_{3}(a, b, c)^{2}=I_{3}=\left(\begin{array}{ccc}
1 & 0 & 0 \\
0 & 1 & 0 \\
0 & 0 & 1
\end{array}\right)
$$

Equation 20.

Define a morphism $A_{3}: \mathbb{C}_{\sim_{1}}^{\times} \times \mathbb{C}^{\times} \times \mathbb{C}^{\times} \rightarrow M_{3}(\mathbb{C})$ by

$$
A_{3}(a, b, c)=\left(\begin{array}{cc}
(1) & \left(\begin{array}{cc}
c & \frac{-b c}{1-a}
\end{array}\right) \\
\left(\begin{array}{l}
0 \\
0
\end{array}\right) & A_{2}(a, b)
\end{array}\right) .
$$

Equation 21.

By Equation 20, for any $(a, b, c) \in \mathbb{C}_{\sim_{1}}^{\times} \times \mathbb{C}^{\times} \times \mathbb{C}^{\times}$, we have $\left(A_{3}(a, b, c)\right)^{2}=I_{3}$. Now let $n=4$ and consider a matrix $A_{4}(a, b, c, d)$ defined by

$$
A_{4}(a, b, c, d)=\left(\begin{array}{cccc}
1 & 0 & d & \frac{-b d}{1-a} \\
0 & 1 & c & \frac{-b c}{1-a} \\
0 & 0 & a & b \\
0 & 0 & \frac{1-a^{2}}{b} & -a
\end{array}\right) \in M_{4}(\mathbb{C}),
$$

Equation 22.

for $a, b, c, d \in \mathbb{C}^{\times}$. Similarly, this matrix $A_{4}(a, b, c, d)$ is regarded as the block matrix,

$$
\begin{aligned}
A_{4}(a, b, c, d) & =\left(\begin{array}{ccc}
(1) & \left(\begin{array}{ccc}
0 & d & \frac{-b d}{1-a}
\end{array}\right) \\
\left(\begin{array}{c}
0 \\
0 \\
0
\end{array}\right) & & A_{3}(a, b, c)
\end{array}\right) \\
A_{4} & =\left(\begin{array}{cc}
I_{2} & \left(\begin{array}{cc}
d & \frac{-b d}{1-a} \\
c & \frac{-b c}{1-a} \\
O_{2,2} & A_{2}(a, b)
\end{array}\right) .
\end{array}\right.
\end{aligned}
$$

Equation 23. 
in $M_{4}(\mathbb{C})$, where $O_{n, m}$ is the $(n \times m)$-zero matrix. Then, by the direct computation, one obtains that

$$
A_{4}^{2}=I_{4}
$$

Equation 24.

Similar to Equation 22, we define a morphism $A_{4}: \mathbb{C}_{\sim_{1}}^{\times} \times\left(\mathbb{C}^{\times}\right)^{3} \rightarrow M_{4}(\mathbb{C})$ by

$$
A_{4}(a, b, c, d)=\left(\begin{array}{cc}
I_{2} & Q_{a, b}(c, d) \\
O_{2,2} & A_{2}(a, b)
\end{array}\right),
$$

Equation 25.

where

$$
Q_{a, b}(c, d)=\left(\begin{array}{cc}
d & \frac{-b d}{1-a} \\
c & \frac{-b c}{1-a}
\end{array}\right)
$$

Equation 26.

The image $A_{4}(a, b, c, d)$ is a well-defined matrix in $M_{4}(\mathbb{C})$, by Equations 22 and 23. Moreover, by Equation 24, we have

$$
\left(A_{4}(a, b, c, d)\right)^{2}=I_{4} \in M_{4}(\mathbb{C}) .
$$

Equation 27.

Let's consider one more step: let $n=5$. Similar to the above, we define the following map. $A_{5}: \mathbb{C}_{\sim_{1}}^{\times} \times\left(\mathbb{C}^{\times}\right)^{4} \rightarrow M_{5}(\mathbb{C})$ by

$$
A_{5}\left(a_{1}, \ldots, a_{5}\right)=\left(\begin{array}{cc}
I_{3} & Q_{a_{1}, a_{2}}\left(a_{3}, a_{4}, a_{5}\right) \\
O_{2,3} & A_{2}\left(a_{1}, a_{2}\right)
\end{array}\right),
$$

Equation 28.

for all $\left(a_{1}, \ldots, a_{5}\right) \in\left(\mathbb{C}^{\times}\right)^{5}$, where

$$
Q_{a_{1}, a_{2}}\left(a_{3}, a_{4}, a_{5}\right)=\left(\begin{array}{cc}
a_{5} & \frac{-a_{2} a_{5}}{1-a_{1}} \\
a_{4} & \frac{-a_{2} a_{4}}{1-a_{1}} \\
a_{3} & \frac{-a_{2} a_{3}}{1-a_{1}}
\end{array}\right)
$$

Equivalently,

$$
A_{5}\left(a_{1}, \ldots, a_{5}\right)=\left(\begin{array}{ccccc}
1 & 0 & 0 & a_{5} & \frac{-a_{2} a_{5}}{1-a_{1}} \\
0 & 1 & 0 & a_{4} & \frac{-a_{2} a_{4}}{1-a_{1}} \\
0 & 0 & 1 & a_{3} & \frac{-a_{2} a_{3}}{1-a_{1}} \\
0 & 0 & 0 & a_{1} & a_{2} \\
0 & 0 & 0 & \frac{1-a_{1}^{2}}{a_{2}} & -a_{1}
\end{array}\right) \in M_{5}(\mathbb{C})
$$

Equation 30.

From direct computation, one again obtains that

$$
\left(A_{5}\left(a_{1}, \ldots, a_{5}\right)\right)^{2}=I_{5} .
$$

Equation 31.

Inductively, for $n \geq 3$, we define the following map $A_{n}: \mathbb{C}_{\sim_{1}}^{\times} \times\left(\mathbb{C}^{\times}\right)^{n-1} \rightarrow M_{n}(\mathbb{C})$, by

$$
A_{n}\left(a_{1}, \ldots, a_{n}\right)=\left(\begin{array}{cc}
I_{n-2} & Q_{a_{1}, a_{2}}\left(a_{3}, \ldots, a_{n}\right) \\
O_{2, n-2} & A_{2}\left(a_{1}, a_{2}\right)
\end{array}\right),
$$

Equation 32.

for all $\left(a_{1}, \ldots, a_{n}\right) \in\left(\mathbb{C}^{\times}\right)^{n}$, where $A_{2}\left(a_{1}, a_{2}\right)$ is in the sense of Equation 6, $O_{2, n-2}$ is the $(2 \times(n-2))$-zero matrix, $I_{n-2}$ is the $(n-2) \times(n-2)$ identity matrix, and

$$
Q_{a_{1}, a_{2}}\left(a_{3}, \ldots, a_{n}\right)=\left(\begin{array}{cc}
a_{n} & \frac{-a_{2} a_{n}}{1-a_{1}} \\
\vdots & \vdots \\
a_{4} & \frac{-a_{2} a_{3}}{1-a_{1}} \\
a_{3} & \frac{-a_{2} a_{3}}{1-a_{1}}
\end{array}\right) .
$$

Then we obtain the following computation. 
Theorem 4. Let $A_{n}$ be the morphism Equation 32, and let $A_{n}\left(a_{1}, \ldots, a_{n}\right)$ be the image of $A_{n}$ realized in the matricial algebra $M_{n}(\mathbb{C})$, for fixed $n \geq 3$ in $\mathbb{N}$. Then

$$
\left(A_{n}\left(a_{1}, \ldots, a_{n}\right)\right)^{2}=I_{n},
$$

Equation 34.

the identity matrix of $M_{n}(\mathbb{C})$.

Proof. Let $n \geq 3$ be given in $\mathbb{N}$.

By Equations 20, 27 and 31, we have $\left(A_{k}\left(a_{1}, \ldots, a_{k}\right)\right)^{2}=I_{k} \in M_{k}(\mathbb{C})$, for $k=3,4,5$.

Now, without loss of generality take $n \geq 6$ in $\mathbb{N}$. Then

$$
A_{n}\left(a_{1}, \ldots, a_{n}\right)=\left(\begin{array}{cc}
I_{n-2} & Q_{a_{1}, a_{2}}\left(a_{3}, \ldots, a_{n}\right) \\
O_{2, n-2} & A_{2}\left(a_{1}, a_{2}\right)
\end{array}\right) .
$$

For convenience, we let

$$
\begin{aligned}
& I \stackrel{\text { denote }}{=} I_{n-2} \\
& Q \stackrel{\text { denote }}{=} Q_{a_{1}, a_{2}}\left(a_{3}, \ldots, a_{n}\right) \\
& O \stackrel{\text { denote }}{=} O_{2, n-2} \\
& A \stackrel{\text { denote }}{=} A_{2}\left(a_{1}, a_{2}\right)
\end{aligned}
$$

Equation 36.

So

$$
\left(\begin{array}{ll}
I & Q \\
O & A
\end{array}\right) \stackrel{\text { denote }}{=} A_{n}\left(a_{1}, \ldots, a_{n}\right)
$$

Equation 37.

as a block matrix in $M_{n}(\mathbb{C})$. Then

$$
\begin{aligned}
\left(A_{n}\left(a_{1}, \ldots, a_{n}\right)\right)^{2} & =\left(\begin{array}{cc}
I & Q \\
O & A
\end{array}\right)\left(\begin{array}{cc}
I & Q \\
O & A
\end{array}\right) \\
& =\left(\begin{array}{cc}
I^{2}+Q O & I Q+Q A \\
O I+A O & O Q+A^{2}
\end{array}\right) \\
& =\left(\begin{array}{cc}
I & I Q+Q A \\
O & A^{2}
\end{array}\right) \\
& =\left(\begin{array}{cc}
I & Q+Q A \\
O & I_{2}
\end{array}\right),
\end{aligned}
$$

Equation 38 .

by Equation 7. So, to show Equation 34, it is sufficient to show that $Q+Q A=O_{n-2,2}$. 
Notice that

$$
\begin{aligned}
Q A & =\left(\begin{array}{cc}
a_{n} & \frac{-a_{2} a_{n}}{1-a_{1}} \\
\vdots & \vdots \\
a_{4} & \frac{-a_{2} a_{4}}{1-a_{1}} \\
a_{3} & \frac{-a_{2} a_{3}}{1-a_{1}}
\end{array}\right)\left(\begin{array}{cc}
a_{1} & a_{2} \\
\frac{1-a_{1}^{2}}{a_{2}} & -a_{1}
\end{array}\right) \\
& =\left(\begin{array}{cc}
a_{1} a_{n}+\frac{-a_{2} a_{n}\left(1-a_{1}^{2}\right)}{a_{2}\left(1-a_{1}\right)} & a_{2} a_{n}+\frac{a_{1} a_{2} a_{n}}{1-a_{1}} \\
\vdots & \vdots \\
a_{1} a_{3}+\frac{-a_{2} a_{3}\left(1-a_{1}^{2}\right)}{a_{2}\left(1-a_{1}\right)} & a_{2} a_{3}+\frac{a_{1} a_{2} a_{3}}{1-a_{1}}
\end{array}\right) \\
& =\left(\begin{array}{cc}
-a_{n} & a_{2} a_{n}\left(1+\frac{a_{1}}{1-a_{1}}\right) \\
\vdots & \vdots \\
-a_{3} & a_{2} a_{3}\left(1+\frac{a_{1}}{1-a_{1}}\right)
\end{array}\right) .
\end{aligned}
$$

Equation 39.

Thus,

$$
\begin{aligned}
Q+Q A & =\left(\begin{array}{cc}
a_{n} & \frac{-a_{2} a_{n}}{1-a_{1}} \\
\vdots & \vdots \\
a_{3} & \frac{-a_{2} a_{3}}{1-a_{1}}
\end{array}\right)+\left(\begin{array}{cc}
-a_{n} & a_{2} a_{n}\left(1+\frac{a_{1}}{1-a_{1}}\right) \\
\vdots & \\
-a_{3} & a_{2} a_{3}\left(1+\frac{a_{1}}{1-a_{1}}\right)
\end{array}\right) \\
& =\left(\begin{array}{cc}
0 & a_{2} a_{n}\left(\frac{-1}{1-a_{1}}+1+\frac{a_{1}}{1-a_{1}}\right) \\
\vdots & \vdots \\
0 & a_{2} a_{3}\left(\frac{-1}{1-a_{1}}+1+\frac{a_{1}}{1-a_{1}}\right)
\end{array}\right) \\
& =\left(\begin{array}{cc}
0 & 0 \\
\vdots & \vdots \\
0 & 0
\end{array}\right) \\
& =O_{n-2,2},
\end{aligned}
$$

Equation 40.

So, for any $n \geq 6$,

$$
\left(A_{n}\left(a_{1}, \ldots, a_{n}\right)\right)^{2}=\left(\begin{array}{cc}
I & Q+Q A \\
O & I
\end{array}\right)=\left(\begin{array}{cc}
I & O_{n-2,2} \\
O & I
\end{array}\right)=I_{n} .
$$

Equation 41.

$n$-Dimensional Hilbert-Space Representations of $\Gamma_{N}^{2}$

Fix $N \in \mathbb{N}$ and $n \in \mathbb{N} \backslash\{1\}$. Let $\Gamma_{N}^{2}$ be our finitely presented group as defined in Equation 1. For the fixed $n$, we take the $n$-tuples

$$
W_{k}=\left(a_{k, 1}, \ldots, a_{k, n}\right) \in \mathbb{C}_{{ }_{1}}^{\times} \times\left(\mathbb{C}^{\times}\right)^{n-1},
$$

Equation 42.

for $k=1, \ldots, N$, and assume that $W_{1}, \ldots, W_{N}$ are mutually distinct.

Observation and Notation As we assumed above, let $W_{1}, \ldots, W_{N}$ be mutually distinct in $\mathbb{C}_{\sim}^{\times} \times\left(\mathbb{C}^{\times}\right)^{n-1}$. For our purposes, one may further assume that these mutually distinct $n$-tuples satisfy $W_{i} \nsucceq W_{j}$ in the sense that $a_{i, l} \neq a_{j, l} \in \mathbb{C}^{\times}$, for all $l=1, \ldots, N$. If the $n$-tuples $W_{1}, \ldots, W_{N}$ satisfy the above stronger condition than the mutually-distinctness, we say they are strongly mutually distinct. Define the corresponding matrices,

$$
X_{k} \stackrel{\text { denote }}{=} A_{n}\left(W_{k}\right)=\left(\begin{array}{cc}
I_{n-2} & Q_{a_{k, 1}, a_{k, 2}}\left(a_{k, 3}, \ldots, a_{k, n}\right) \\
O_{2, n-2} & A_{2}\left(a_{k, 1}, a_{k, 2}\right)
\end{array}\right),
$$

Equation 43. 
as in Equation 32, where $W_{k}$ are strongly mutually distinct $n$-tuples of Equation $\mathbf{4 2}$, for all $k=1, \ldots, N$.

Then, one can create the multiplicative subgroup $\mathfrak{M}_{n}^{2}(N)$ of $M_{n}(\mathbb{C})$ from the (reduced) free group generated by $\left\{X_{1}, \ldots\right.$, $\left.X_{N}\right\}: \mathfrak{M}_{n}^{2}(N)=\left\langle\left\{X_{j}\right\}_{j=1}^{N}\right\rangle \in M_{n}(\mathbb{C})$.

Definition 2. We call the multiplicative subgroup $\mathfrak{M}_{n}^{2}(N)$ of $M_{n}(\mathbb{C})$, an n-dimensional, order-2, $N$-generator (sub-)group (of $\left.M_{n}(\mathbb{C})\right)$.

Then, similar to the proof of the structure theorem Equation 14, one can obtain the following generalized result.

Theorem 5. Let $n \in \mathbb{N} \backslash\{1\}$ and $\mathfrak{M}_{n}^{2}(N)$ be an n-dimensional, order-2, $N$-generator group in $M_{n}(\mathbb{C})$. Then

$$
\mathfrak{M}_{n}^{2}(N) \stackrel{\text { Group }}{=} \Gamma_{N}^{2}
$$

Equation 44.

In particular, there exist generator-preserving group isomorphisms $\alpha^{n}: \Gamma_{N}^{2} \rightarrow \mathfrak{M}_{n}^{2}(N)$, such that

$$
\alpha^{n}\left(x_{j}\right)=X_{j} \text {, for all } j=1, \ldots, N,
$$

Equation 45.

where $x_{j}$ 's are the generators of $\Gamma_{N}^{2}$.

Proof. Let $n=2$. Then, by Equation 14, the isomorphic relation Equation 44 holds. Assume now that $n \geq 3$. Then the proof of Equation 44 is similar to that of Equation 14. Indeed, one can show that $\mathfrak{M}_{n}^{2}(N) \stackrel{\text { Group }}{=} \mathcal{F}_{N} / \mathcal{R}_{N} \stackrel{\text { Group }}{=} \Gamma_{N}^{2}$, for all $n \in \mathbb{N}$. Therefore, there exists a natural generator-preserving group-isomorphism $\alpha^{n}$ from $\Gamma_{N}^{2}$ onto $\mathfrak{M}_{n}^{2}(N)$ as in Equation 45.

This structure theorem is the generalized result of Equation 14. And the group-isomorphism $\alpha^{n}$ of Equation 45 generalizes $\alpha^{2}$ of Equation 17. We obtain the following theorem, generalizing Equation 3.

Theorem 6. Let $\Gamma_{N}^{2}$ be the group defined in Equation 1, for some $N \in \mathbb{N}$. Then there exists an n-dimensional Hilbert-space representation $\left(\mathbb{C}^{n}, \alpha^{n}\right)$ of $\Gamma_{N}^{2}$, where $\alpha^{n}$ are in the sense of Equation 45 , for all $n \in \mathbb{N} \backslash\{1\}$.

Proof. If $n=2$, as we have seen in Equation 3, there exists a 2-dimensional Hilbert-space representation $\left(\mathbb{C}^{2}, \alpha^{2}\right)$, where $\alpha^{2}$ is the group-isomorphism in the sense of Equation 17.

Suppose $n \geq 3$ in $\mathbb{N}$. For such $n$, two groups $\Gamma_{N}^{2}$ and $\mathfrak{M}_{n}^{2}(N)$ are isomorphic via a group-isomorphism $\alpha^{n}: \Gamma_{N}^{2} \rightarrow \mathfrak{M}_{n}^{2}(N)$ of Equation 45, by the previous theorem. It shows that the images $\alpha^{n}(g)$ are $(n \times n)$-matrices acting on the $n$-dimensional Hilbert space $\mathbb{C}^{n}$. So, the pair $\left(\mathbb{C}^{n}, \alpha^{n}\right)$ forms an $n$-dimensional representation of $\Gamma_{N}^{2}$, for all $n \in \mathbb{N}$.

The above two theorems show that, for the fixed group $\Gamma_{N}^{2}$, one has a system $\left\{\left(\mathbb{C}^{n}, \alpha^{n}\right)\right\}_{n=2}^{\infty}$ of Hilbert-space representations, and the corresponding isomorphic groups $\left\{\mathfrak{M}_{n}^{2}(N)\right\}_{n=2}^{\infty}$, acting on $\mathbb{C}^{n}$.

Example 1. Let $\Gamma_{2}^{2}$ be the finitely presented group, $\Gamma_{2}^{2}=\left\langle\left\{x_{1}, x_{2}\right\},\left\{x_{1}^{2}=x_{2}^{2}\right\}\right\rangle$. Fix $n=3$. Now, take the following strongly distinct triples, $W_{1}=\left(t_{1}, t_{2}, t_{3}\right), W_{2}=\left(s_{1}, s_{2}, s_{3}\right)$, in $\mathbb{C}_{\sim_{1}}^{\times} \times\left(\mathbb{C}^{\times}\right)^{2}$, and construct two matrices,

$$
A_{3}\left(W_{1}\right)=\left(\begin{array}{ccc}
1 & t_{3} & \frac{-t_{2} t_{3}}{1-t_{1}} \\
0 & t_{1} & t_{2} \\
0 & \frac{1-t_{1}^{2}}{t_{2}} & -t_{1}
\end{array}\right), A_{3}\left(W_{2}\right)=\left(\begin{array}{ccc}
1 & s_{3} & \frac{-s_{2} s_{3}}{1-s_{1}} \\
0 & s_{1} & s_{2} \\
0 & \frac{1-s_{1}^{2}}{s_{2}} & -s_{1}
\end{array}\right),
$$

Equation 46.

in $M_{3}(\mathbb{C})$. The group $\mathfrak{M}_{3}^{2}(2)$ is established as the reduced free group $\left\langle\left\{A_{3}\left(W_{1}\right), A_{3}\left(W_{2}\right)\right\}\right\rangle$ generated by $A_{3}\left(W_{1}\right)$ and $A_{3}\left(W_{2}\right)$ in $M_{3}(\mathbb{C})$. Then $\mathfrak{M}_{3}^{2}(2) \stackrel{\text { Group }}{=} \Gamma_{2}^{2}$.

So, one has a natural 3 -dimensional representation $\left(\mathbb{C}^{3}, \alpha^{3}\right)$, where $\alpha^{3}$ is the group-isomorphism satisfying $\alpha^{3}\left(x_{l}\right)=A_{3}\left(W_{l}\right)$, for all $l=1,2$. 


\section{APPLICATION: A GROUP ALGEBRA $\mathcal{A}_{2}$ INDUCED BY $\Gamma_{2}^{2}$}

In the previous section, we showed that the finitely presented group $\Gamma_{N}^{2}$ has a family of finite-dimensional Hilbert-space representations $\left\{\left(\mathbb{C}^{n}, \alpha^{n}\right)\right\}_{n=2}^{\infty}$, since it is group-isomorphic to $n$-dimensional, order-2, $N$-generator groups $\mathfrak{M}_{n}^{2}(N)$ in $M_{n}(\mathbb{C})$, for all $n \in \mathbb{N} \backslash\{1\}$, where $\mathfrak{M}_{n}^{2}(N)=\left\langle\left\{A_{n}\left(W_{k}\right)\right\}_{k=1}^{N}\right\rangle \in M_{n}(\mathbb{C})$, and where $W_{1}, \ldots, W_{N}$ are strongly mutually distinct $n$-tuples of $\mathbb{C}_{\tilde{v}_{1}}^{\times} \times\left(\mathbb{C}^{\times}\right)^{n-1}$, for $n \in \mathbb{N} \backslash\{1\}$.

Algebraic Observation for Group Elements of $\Gamma_{2}^{2}$

In this section, we concentrate on the case where $N=2$, studying the group elements of $\Gamma_{2}^{2}$ in detail. As a special case of Equation 44,

$$
\left\langle\left\{x_{1}, x_{2}\right\},\left\{x_{1}^{2}=x_{2}^{2}=e_{2}\right\}\right\rangle=\Gamma_{2}^{2} \stackrel{\text { Group }}{=} \mathfrak{M}_{2}^{2}(2) . \quad \text { Equation } 47 .
$$

Consider $\Gamma_{2}^{2}$ pure-algebraically. Each element $g$ of $\Gamma_{2}^{2}$ has its expression,

$$
g=x_{i_{1}}^{k_{1}} x_{i_{2}}^{k_{2}} \cdots x_{i_{n}}^{k_{i_{n}}}, \text { for some } n \in \mathbb{N},
$$

Equation 48 .

as in Equation 4, for some $\left(i_{1}, \ldots, i_{n}\right) \in\{1,2\}^{n}$, and $\left(k_{1}, \ldots, k_{n}\right) \in \mathbb{Z}^{n}$. However, by the relation on $\Gamma_{2}^{2}, x_{1}^{2}=e \stackrel{\text { denote }}{=}$ $e_{2}=x_{2}^{2} \in \Gamma_{2}^{2}$, one has that $x_{l}^{-1}=x_{l}$, for $l=1,2$, and $x_{l}^{2 k+1}=x_{l}$, for all $k \in \mathbb{N}$. In other words, the generators $x_{1}$ and $x_{2}$ are self-invertible, and indeed of order- 2 , in $\Gamma_{2}^{2}$. The above two conditions in can be summarized by

$$
x_{l}^{2 n+1}=x_{l}, \text { for } l=1,2 \text {, and for } n \in \mathbb{Z} .
$$

Equation 49.

So, the general expression of Equation 48 of $g$ is in fact

$$
g=x_{j_{1}} x_{j_{2}} \ldots x_{j_{n}} \in \Gamma_{2}^{2},
$$

Equation 50.

for some $\left(j_{1}, \ldots, j_{n}\right) \in\{1,2\}^{n}, n \in \mathbb{N}$, by Equation 49 .

By the characterization, $\Gamma_{2}^{2} \stackrel{\text { Group }}{=} \mathcal{F}_{2} / \mathcal{R}_{2}$, and by the definition of the noncommutative (non-reduced) free group $\mathcal{F}_{2}$, the expression Equation $\mathbf{5 0}$ of $g$ goes to

$$
g=\left\{\begin{array}{l}
e=e_{2} \\
x_{1} \\
x_{2} \\
\left(x_{1} x_{2}\right)^{n} x_{1} \\
\left(x_{1} x_{2}\right)^{n} \\
\left(x_{2} x_{1}\right)^{n} x_{2} \\
\left(x_{2} x_{1}\right)^{n}
\end{array}\right.
$$

Equation 51.

in $\Gamma_{2}^{2}$, for all $n \in \mathbb{N}$.

Proposition 7. Let $g \in \Gamma_{2}^{2}$. Then $g$ is only one of the forms in Equation 51.

Proof. Let $g \in \Gamma_{2}^{2} \backslash\left\{e, x_{1}, x_{2}\right\}$. Say, $g=x_{1} x_{2} x_{1} x_{2} \ldots x_{1} x_{2} x_{1}$. Then it is self-invertible. Indeed,

$$
\begin{aligned}
g^{2} & =\left(x_{1} x_{2} \ldots x_{1} x_{2} x_{1}\right)\left(x_{1} x_{2} \ldots x_{1} x_{2} x_{1}\right) \\
& =x_{1} x_{2} \ldots x_{1} x_{2}\left(x_{1}^{2}\right) x_{2} \ldots x_{1} x_{2} x_{1} \\
& =x_{1} x_{2} \ldots x_{1} x_{2} e x_{2} \ldots x_{1} x_{2} x_{1} \\
& =x_{1} x_{2} \ldots x_{1}\left(x_{2}^{2}\right) x_{1} \ldots x_{1} x_{2} x_{1} \\
& \vdots \\
& =x_{1}^{2} \\
& =e .
\end{aligned}
$$

Equation 52. 
So

$$
\left(\left(x_{1} x_{2}\right)^{n} x_{1}\right)^{2}=e, \text { for all } n \in \mathbb{N}
$$

Equation 53.

Similarly, one obtains

$$
\left(\left(x_{2} x_{1}\right)^{n} x_{2}\right)^{2}=e, \text { for all } n \in \mathbb{N} .
$$

Equation 54.

Now, let $g=x_{1} x_{2} x_{1} x_{2} \ldots x_{1} x_{2} \in \Gamma_{2}^{2}$. Then $g^{-1}=x_{2} x_{1} x_{2} x_{1} \ldots x_{2} x_{1}$, with $|g|=\left|g^{-1}\right| \in \mathbb{N}$, where $|w|$ means the length of the word $w \in \Gamma_{2}^{2}$. Indeed, $g g^{-1}=\left(x_{1} x_{2} \ldots x_{1} x_{2}\right)\left(x_{2} x_{1} \ldots x_{2} x_{1}\right)=e$. So, one has that

$$
\left(\left(x_{1} x_{2}\right)^{n}\right)^{-1}=\left(x_{2} x_{1}\right)^{n},
$$

Equation 55.

and equivalently,

$$
\left(\left(x_{2} x_{1}\right)^{n}\right)^{-1}=\left(x_{1} x_{2}\right)^{n},
$$

Equation 56.

for all $n \in \mathbb{N}$.

Proposition 8. Let $g \in \Gamma_{2}^{2} \backslash\{e\}$, and let $|g|$ be the length of $g$ in $\left\{x_{1}, x_{2}\right\}$ in $\Gamma_{2}^{2}$.

1. If $|g|$ is odd then $g^{-1}=g$ in $\Gamma_{2}^{2}$.

2. If $|g|$ is even then $g=\left(x_{1} x_{2}\right)^{k}$, or $\left(x_{2} x_{1}\right)^{k}$, and $g^{-1}=\left(x_{2} x_{1}\right)^{k}$, respectively, $\left(x_{1} x_{2}\right)^{k}$, for some $k \in \mathbb{N}$.

Proof. Suppose $|g|$ is odd in $\mathbb{N}$. Then, by Equation 51, the group element $g$ is one of $x_{1}, x_{2},\left(x_{1} x_{2}\right)^{n} x_{1}$, or $\left(x_{2} x_{1}\right)^{n} x_{2}$, for $n \in \mathbb{N}$. By Equation 53 and Equation 54, in such cases, $g^{2}=e \in \Gamma_{2}^{2}$. So, the first statement holds.

Now, assume that $|g|$ is even in $\mathbb{N}$. Then, by Equation 51, this element $g$ is either $\left(x_{1} x_{2}\right)^{k}$, or $\left(x_{2} x_{1}\right)^{k}$, for $k \in \mathbb{N}$. By Equation 55 and Equation 56, respectively one has that

$$
\left(\left(x_{1} x_{2}\right)^{k}\right)^{-1}=\left(x_{2} x_{1}\right)^{k}, \text { and }\left(\left(x_{2} x_{1}\right)^{k}\right)^{-1}=\left(x_{1} x_{2}\right)^{k},
$$

Equation 57.

for all $k \in \mathbb{N}$. Therefore, the second statement holds, too.

Due to the self-invertibility of the group-identity, $e$, the generators $x_{1}, x_{2}$, and the group elements $g$ with odd length $|g|$, we are interested in the cases where $|g|$ is even: the cases where $g=\left(x_{1} x_{2}\right)^{k}$, or $g=\left(x_{2} x_{1}\right)^{k} \in \Gamma_{2}^{2}$.

Analytic and Combinatorial Observation for Elements of $\Gamma_{2}^{2}$ in $M_{2}(\mathbb{C})$

As we have seen, the group $\Gamma_{2}^{2}$ is group isomorphic to the 2-dimensional, order-2, 2-generator subgroup $\mathfrak{M}_{2}^{2}(2)$ of $M_{2}(\mathbb{C})$ under our representation $\left(\mathbb{C}^{2}, \alpha^{2}\right)$. In particular, $\mathfrak{M}_{2}^{2}(2)$ is generated by two matrices: $A_{2}\left(t_{1}, s_{1}\right)$ and $A_{2}\left(t_{2}, s_{2}\right)$ for the strongly mutually distinct pairs $\left(t_{1}, s_{1}\right)$ and $\left(t_{2}, s_{2}\right)$ in $\mathbb{C}^{\times} \times \mathbb{C}^{\times}$. More precisely,

$$
A_{2}\left(t_{1}, s_{1}\right)=\left(\begin{array}{cc}
t_{1} & s_{1} \\
\frac{1-t_{1}^{2}}{s_{1}} & -t_{1}
\end{array}\right) \text { and } A_{2}\left(t_{2}, s_{2}\right)=\left(\begin{array}{cc}
t_{2} & s_{2} \\
\frac{1-t_{2}^{2}}{s_{2}} & -t_{2}
\end{array}\right)
$$

Equation 58.

in $M_{2}(\mathbb{C})$.

In the rest of this paper, we denote $A_{2}\left(t_{1}, s_{1}\right)$ and $A_{2}\left(t_{2}, s_{2}\right)$, by $X_{1}$ and $X_{2}$, respectively, for convenience.

We are interested in group-elements $g$ formed by $g=\left(x_{1} x_{2}\right)^{k} \in \Gamma_{2}^{2}$, for $k \in \mathbb{N}$. In this case

$$
\alpha^{2}(g)=\alpha^{2}\left(\left(x_{1} x_{2}\right)^{k}\right)=\left(X_{1} X_{2}\right)^{k} \text {, and hence, }\left(\alpha^{2}(g)\right)^{-1}=\alpha^{2}\left(g^{-1}\right)=\left(X_{2} X_{1}\right)^{k},
$$

Equation 59.

in $\mathfrak{M}_{2}^{2}(2)$. 
Observe that

$$
\left(X_{1} X_{2}\right)+\left(X_{2} X_{1}\right)=\left(2 t_{1} t_{2}+\frac{s_{1}\left(1-t_{2}^{2}\right)}{s_{2}}+\frac{s_{2}\left(1-t_{1}^{2}\right)}{s_{1}}\right) I_{2}
$$

from the straightforward computation in $M_{2}(\mathbb{C})$.

Consider now that $\operatorname{det}\left(X_{1}\right)=-1=\operatorname{det}\left(X_{2}\right)$, and

$$
\begin{aligned}
\operatorname{det}\left(X_{1}+X_{2}\right) & =\operatorname{det}\left(\begin{array}{cc}
t_{1}+t_{2} & s_{1}+s_{2} \\
\frac{1-t_{1}^{2}}{s_{1}}+\frac{1-t_{2}^{2}}{s_{2}} & -t_{1}-t_{2}
\end{array}\right) \\
& =-\left(t_{1}^{2}+2 t_{1} t_{2}+t_{2}^{2}\right)-\left(1-t_{1}^{2}+\frac{s_{1}\left(1-t_{2}^{2}\right)}{s_{2}}+\frac{s_{2}\left(1-t_{1}^{2}\right)}{s_{1}}+1-t_{2}^{2}\right) \\
& =-t_{1}^{2}-2 t_{1} t_{2}-t_{2}^{2}-1+t_{1}^{2}-\frac{s_{1}\left(1-t_{2}^{2}\right)}{s_{2}}-\frac{s_{2}\left(1-t_{1}^{2}\right)}{s_{1}}-1+t_{2}^{2} \\
& =-2 t_{1} t_{2}-\frac{s_{1}\left(1-t_{2}^{2}\right)}{s_{2}}-\frac{s_{2}\left(1-t_{1}^{2}\right)}{s_{1}}-2,
\end{aligned}
$$

Equation 61.

So, one can compute

$$
\operatorname{det}\left(X_{1}\right)+\operatorname{det}\left(X_{2}\right)-\operatorname{det}\left(X_{1}+X_{2}\right)=2 t_{1} t_{2}+\frac{s_{1}\left(1-t_{2}^{2}\right)}{s_{2}}+\frac{s_{2}\left(1-t_{1}^{2}\right)}{s_{1}} .
$$

Equation 62.

Lemma 9. Let $X_{1}$ and $X_{2}$ be the generating matrices of $\mathfrak{M}_{2}^{2}(2)$ as above. Then there exists $\varepsilon_{0} \in \mathbb{C}$, such that

$$
\begin{gathered}
\left(X_{1} X_{2}\right)+\left(X_{2} X_{1}\right)=\varepsilon_{0} I_{2} \in M_{2}(\mathbb{C}), \text { and } \\
\varepsilon_{0}=2 t_{1} t_{2}+\frac{s_{1}\left(1-t_{2}^{2}\right)}{s_{2}}+\frac{s_{2}\left(1-t_{1}^{2}\right)}{s_{1}} \\
=\operatorname{det}\left(X_{1}\right)+\operatorname{det}\left(X_{2}\right)-\operatorname{det}\left(X_{1}+X_{2}\right) .
\end{gathered}
$$

Equation 63.

Proof. The proof of Equation 63 is done by Equation 60 and Equation 62.

Therefore, one can observe that

$$
\begin{aligned}
\left(X_{1} X_{2}+X_{2} X_{1}\right)^{2} & =\left(X_{1} X_{2}\right)^{2}+X_{1} X_{2} X_{2} X_{1}+X_{2} X_{1} X_{1} X_{2}+\left(X_{2} X_{1}\right)^{2} \\
& =\left(X_{1} X_{2}\right)^{2}+\left(X_{2} X_{1}\right)^{2}+2 I_{2},
\end{aligned}
$$

and hence,

$$
\begin{aligned}
\left(X_{1} X_{2}\right)^{2}+\left(X_{2} X_{1}\right)^{2} & =\left(X_{1} X_{2}+X_{2} X_{1}\right)^{2}-2 I_{2} \\
& =\left(\varepsilon_{0} I_{2}\right)^{2}-2 I_{2} \\
& =\left(\varepsilon_{0}^{2}-2\right) I_{2} .
\end{aligned}
$$

By Equation 63 and Equation 65, we have that $\left(X_{1} X_{2}\right)^{1}+\left(X_{2} X_{1}\right)^{1}=\varepsilon_{0} I_{2}$, and $\left(X_{1} X_{2}\right)^{2}+\left(X_{2} X_{1}\right)^{2}=\left(\varepsilon_{0}^{2}-2\right) I_{2}$. More generally, we obtain the following recurrence relation. 
Theorem 10. Let $X_{1}$ and $X_{2}$ be the generating matrices of $\mathfrak{M}_{2}^{2}(2)$ in $M_{2}(\mathbb{C})$. If we denote $\mathfrak{X}_{k} \stackrel{\text { denote }}{=}\left(X_{1} X_{2}\right)^{k}+\left(X_{2} X_{1}\right)^{k}$, for all $k \in \mathbb{N}$, then the following recurrence relation is obtained.

$$
\begin{aligned}
& \mathfrak{X}_{1}=\varepsilon_{0} I_{2} \\
& \mathfrak{X}_{2}=\left(\varepsilon_{0}^{2}-2\right) I_{2} \\
& \quad \vdots \\
& \mathfrak{X}_{n}=\varepsilon_{0} \mathfrak{X}_{n-1}-\mathfrak{X}_{n-2}, \text { for all } n \geq 3 \in \mathbb{N},
\end{aligned}
$$

Equation 66.

in $M_{2}(\mathbb{C})$, where $\varepsilon_{0}=\operatorname{det}\left(X_{1}\right)+\operatorname{det}\left(X_{2}\right)-\operatorname{det}\left(X_{1}+X_{2}\right)$.

Proof. By Equation 63, indeed, one has $\mathfrak{X}_{1}=\varepsilon_{0} I_{2}$, and by Equation 65, $\mathfrak{X}_{2}=\left(\varepsilon_{0}^{2}-2\right) I_{2}$.

Suppose $n=3$ in $\mathbb{N}$. Then by Equation 51

$$
\begin{aligned}
\mathfrak{X}_{3} & =\left(X_{1} X_{2}\right)^{3}+\left(X_{2} X_{1}\right)^{3} \\
& =\left(X_{1} X_{2}+X_{2} X_{1}\right)^{3}-\left(3 X_{1} X_{2}+3 X_{2} X_{1}\right) \\
& =\left(\mathfrak{X}_{1}\right)^{3}-3 \mathfrak{X}_{1} \\
& =\left(\varepsilon_{0} I_{2}\right)^{3}-3\left(\varepsilon_{0} I_{2}\right) \\
& =\left(\varepsilon_{0}^{3}-3 \varepsilon_{0}\right) I_{2} .
\end{aligned}
$$

Equation 67.

Observe now that

$$
\begin{aligned}
\varepsilon_{0} \mathfrak{X}_{2}-\mathfrak{X}_{1} & =\varepsilon_{0}\left(\varepsilon_{0}^{2}-2\right) I_{2}-\varepsilon_{0} I_{2} \\
& =\left(\varepsilon_{0}^{3}-2 \varepsilon_{0}\right) I_{2}-\varepsilon_{0} I_{2} \\
& =\left(\varepsilon_{0}^{3}-3 \varepsilon_{0}\right) I_{2} .
\end{aligned}
$$

Equation 68.

Thus, one obtains that

$$
\begin{aligned}
\mathfrak{X}_{3} & =\left(\varepsilon_{0}^{3}-3 \varepsilon_{0}\right) \\
I_{2} & =\varepsilon_{0} \mathfrak{X}_{2}-\mathfrak{X}_{1} .
\end{aligned}
$$

Equation 69.

So, if $n=3$, then the relation in Equation 66 holds true.

Assume now that the statement

$$
\mathfrak{X}_{n_{0}}=\varepsilon_{0} \mathfrak{X}_{n_{0}-1}-\mathfrak{X}_{n_{0}-2}
$$

Equation 70.

holds for a fixed $n_{0} \geq 3$ in $\mathbb{N}$. Then

$$
\begin{aligned}
\mathfrak{X}_{n_{0}+1} & =\left(\left(X_{1} X_{2}\right)^{n_{0}}+\left(X_{2} X_{1}\right)^{n_{0}}\right)\left(X_{1} X_{2}+X_{2} X_{1}\right)-\left(X_{1} X_{2}\right)^{n_{0}}\left(X_{2} X_{1}\right)-\left(X_{2} X_{1}\right)^{n_{0}}\left(X_{1} X_{2}\right) \\
& =\left(\left(X_{1} X_{2}\right)^{n_{0}}+\left(X_{2} X_{1}\right)^{n_{0}}\right)\left(\varepsilon_{0} I\right)-\left(\left(X_{1} X_{2}\right)^{n_{0}-1}+\left(X_{2} X_{1}\right)^{n_{0}-1}\right) \\
& =\varepsilon_{0} \mathfrak{X}_{n_{0}}-\mathfrak{X}_{n_{0}-1},
\end{aligned}
$$

Equation 71.

Since $n_{0}$ is arbitrary in $\mathbb{N} \backslash\{1,2\}$, we can conclude that $\mathfrak{X}_{n+1}=\varepsilon_{0} \mathfrak{X}_{n}-\mathfrak{X}_{n-1}$, for all $n \in \mathbb{N} \backslash\{1\}$, by the induction.

Therefore, the relation in Equation 66 holds. 
By the recurrence relation Equation 66, we obtain that:

$$
\begin{aligned}
X_{1} X_{2}+X_{2} X_{1} & =\varepsilon_{0} I_{2}, \\
\left(X_{1} X_{2}\right)^{2}+\left(X_{2} X_{1}\right)^{2} & =\left(\varepsilon_{0}^{2}-2\right) I_{2}, \\
\left(X_{1} X_{2}\right)^{3}+\left(X_{2} X_{1}\right)^{3} & =\left(\varepsilon_{0}^{3}-3 \varepsilon_{0}\right) I_{2}, \\
\left(X_{1} X_{2}\right)^{4}+\left(X_{2} X_{1}\right)^{4} & =\varepsilon_{0}\left(\varepsilon_{0}^{3}-3 \varepsilon_{0}\right) I_{2}-\left(\varepsilon_{0}^{2}-2\right) I_{2} \\
& =\left(\varepsilon_{0}^{4}-4 \varepsilon_{0}^{2}+2\right) I_{2} .
\end{aligned}
$$

Equation 72.

Inductively, one can verify the following.

Corollary 11. There exists a functional sequence $\left(f_{n}\right)_{n=1}^{\infty}$, such that

$$
\left(X_{1} X_{2}\right)^{n}+\left(X_{2} X_{1}\right)^{n}=\left(f_{n}\left(\varepsilon_{0}\right)\right) I_{2} .
$$

Equation 73 .

The following theorem provides the refined result of Equation 73.

Theorem 12. Let $X_{1}$ and $X_{2}$ be the generating matrices of a 2-dimensional, order-2, 2-generator subgroup $\mathfrak{M}_{2}^{2}(2)$ of $M_{2}(\mathbb{C})$, and let $\mathfrak{X}_{l}=\left(X_{1} X_{2}\right)^{l}+\left(X_{2} X_{1}\right)^{l} \in M_{2}(\mathbb{C})$, for all $l \in \mathbb{N}$. Then there exists a functional sequence $\left(f_{n}\right)_{n=1}^{\infty}$ such that $\mathfrak{X}_{n}=$ $f_{n}\left(\varepsilon_{0}\right) I_{2} \in M_{2}(\mathbb{C})$, where $\varepsilon_{0}=\operatorname{det}\left(X_{1}\right)+\operatorname{det}\left(X_{2}\right)-\operatorname{det}\left(X_{1}+X_{2}\right)$. Moreover,

$$
\begin{aligned}
f_{n}(z) & =\sum_{k=0}^{\left[\frac{n}{2}\right]}(-1)^{k} \frac{n}{k}\left(\begin{array}{c}
n-k-1 \\
k-1
\end{array}\right) z^{n-2 k} \\
& =\sum_{k=0}^{\left[\frac{n}{2}\right]}(-1)^{k} \frac{n}{n-k}\left(\begin{array}{c}
n-k \\
k
\end{array}\right) z^{n-2 k}
\end{aligned}
$$

Equation 74.

where $\left[\frac{n}{2}\right]$ is the maximal integer less than or equal to $\frac{n}{2}$.

Proof. By Equation 66 and Equation 73, there exists a functional sequence $\left(f_{n}\right)_{n=1}^{\infty}$ such that $\mathfrak{X}_{n}=f_{n}\left(\varepsilon_{0}\right) I_{2}$, for all $n \in \mathbb{N}$. For instance,

$$
\begin{aligned}
& f_{1}(z)=z \\
& f_{2}(z)=z^{2}-2 \\
& f_{3}(z)=z^{3}-3 z \\
& f_{4}(z)=z^{4}-4 z^{2}+2
\end{aligned}
$$

Equation 75.

So, it suffices to show that each $n$-th entry of the sequence $f_{n}(z)$ satisfies Equation 74 , for all $n \in \mathbb{N}$.

Say $n=1$. The function $f_{1}(z)$ satisfies

$$
\begin{aligned}
f_{1}(z) & =\sum_{k=0}^{\left[\frac{1}{2}\right]}(-1)^{k} \frac{n}{n-k}\left(\begin{array}{c}
n-k-1 \\
k-1
\end{array}\right) z^{n-2 k} \\
& =\sum_{k=0}^{0}(-1)^{k} \frac{n}{n-k}\left(\begin{array}{c}
n-k \\
k
\end{array}\right) z^{n-2 k} \\
& =(-1)^{0} \frac{1}{1-0}\left(\begin{array}{c}
1-0 \\
0
\end{array}\right) z^{1-0} \\
& =z
\end{aligned}
$$

Equation 76. 
If $n=2$, then the function $f_{2}(z)$ is

$$
\begin{aligned}
f_{2}(z) & =\sum_{k=0}^{1}(-1)^{k} \frac{n}{n-k}\left(\begin{array}{c}
n-k \\
k
\end{array}\right) z^{n-2 k} \\
& =(-1)^{0} \frac{2}{2-0}\left(\begin{array}{c}
2-0 \\
0
\end{array}\right) z^{2-0}+(-1)^{1} \frac{2}{2-1}\left(\begin{array}{c}
2-1 \\
1
\end{array}\right) z^{2-2} \\
& =z^{2}-1
\end{aligned}
$$

Equation 77.

Therefore, the formula Equation 74 holds true where $n=1,2$, by Equation 75 .

Now, we consider the recurrence relation.

$$
\begin{aligned}
f_{1}(z) & =z \\
f_{2}(z) & =z^{2}-1 \\
f_{n+1}(z) & =z f_{n}(z)-f_{n-1}(z)
\end{aligned}
$$

Equation 78 .

for all $n \geq 2$ in $\mathbb{N}$.

First of all, the functions $f_{1}$ and $f_{2}$ satisfy the initial condition of the relation by Equation 76 and Equation 77 . So, concentrate on the cases where $n \geq 2$. Observe that

$$
\begin{aligned}
& z f_{n}(z)-f_{n-1}(z)=z\left(\sum_{k=0}^{\left[\frac{n}{2}\right]}(-1)^{k} \frac{n}{k}\left(\begin{array}{c}
n-k-1 \\
k-1
\end{array}\right) z^{n-2 k}\right)-\sum_{k=0}^{\left[\frac{n-1}{2}\right]}(-1)^{k} \frac{n-1}{k}\left(\begin{array}{c}
n-k-2 \\
k-1
\end{array}\right) z^{n-1-2 k} \\
& =\sum_{k=0}^{\left[\frac{n}{2}\right]}(-1)^{k} \frac{n}{k}\left(\begin{array}{c}
n-k-1 \\
k-1
\end{array}\right) z^{n-2 k+1}-\sum_{k=1}^{\left[\frac{n+1}{2}\right]}(-1)^{k-1} \frac{n-1}{k-1}\left(\begin{array}{c}
n-k-1 \\
k-2
\end{array}\right) z^{n-1-2 k+2} \\
& =\sum_{k=0}^{\left[\frac{n}{2}\right]}(-1)^{k} \frac{n}{k}\left(\begin{array}{c}
n-k-1 \\
k-1
\end{array}\right) z^{n+1-2 k}+\sum_{k=1}^{\left[\frac{n+1}{2}\right]}(-1)^{k} \frac{n-1}{k-1}\left(\begin{array}{c}
n-k-1 \\
k-2
\end{array}\right) z^{n+1-2 k} \\
& =z^{n+1}+\sum_{k=1}^{\left[\frac{n}{2}\right]}(-1)^{k} \frac{n}{k}\left(\begin{array}{c}
n-k-1 \\
k-1
\end{array}\right) z^{n+1-2 k}+\sum_{k=1}^{\left[\frac{n+1}{2}\right]}(-1)^{k} \frac{n-1}{k-1}\left(\begin{array}{c}
n-k-1 \\
k-2
\end{array}\right) z^{n+1-2 k} \\
& =z^{n+1}+\sum_{k=1}^{\left[\frac{n}{2}\right]}(-1)^{k}\left(\frac{n}{k}\left(\begin{array}{c}
n-k-1 \\
k-1
\end{array}\right)+\frac{n-1}{k-1}\left(\begin{array}{c}
n-k-1 \\
k-2
\end{array}\right)\right) z^{n+1-2 k} \\
& +(-1)^{\left[\frac{n+1}{2}\right]} \frac{n-1}{\left[\frac{n+1}{2}\right]-1}\left(\begin{array}{c}
n-\left[\frac{n+1}{2}\right]-1 \\
{\left[\frac{n+1}{2}\right]-1}
\end{array}\right) z^{n+1-2\left[\frac{n+1}{2}\right]} \\
& =z^{n+1}+\sum_{k=1}^{\left[\frac{n}{2}\right]}(-1)^{k}\left(\frac{n(n-k-1) !}{k(n-k-1-k+1) !(k-1) !}+\frac{(n-1)(n-k-1) !}{(k-1)(n-k+k+2) !(k-2) !}\right) z^{n+1-2 k} \\
& +\mathfrak{Y} \\
& \text { Equation } 79 .
\end{aligned}
$$


where

$$
\begin{aligned}
& \mathfrak{Y}=(-1)^{\left[\frac{n+1}{2}\right]} \frac{n-1}{\left[\frac{n+1}{2}\right]-1}\left(\begin{array}{c}
n-\left[\frac{n+1}{2}\right]-1 \\
{\left[\frac{n+1}{2}\right]-1}
\end{array}\right) z^{n+1-2\left[\frac{n+1}{2}\right]}, \\
&=z^{n+1}+\sum_{k=1}^{\left[\frac{n}{2}\right]}(-1)^{k}\left(\frac{n(n-k-1) !}{(n-2 k) ! k !}+\frac{n(n-k-1) !-(n-k-1) !}{(n-2 k+1) !(k-1) !}\right) z^{n+1-2 k}+\mathfrak{Y} \\
&=z^{n+1}+\sum_{k=1}^{\left[\frac{n}{2}\right]}(-1)^{k}\left(\frac{(n-2 k+1) n(n-k-1) !+k n(n-k-1) !-k(n-k-1) !}{k(n-2 k+1) !(k-1) !}\right) z^{n+1-2 k}+\mathfrak{Y} \\
&=z^{n+1}+\sum_{k=1}^{\left[\frac{n}{2}\right]}(-1)^{k}\left(\frac{\left(n^{2}-2 k n+n+n k-k\right)(n-k-1) !}{k(n-2 k+1) !(k-1) !}\right) z^{n+1-2 k}+\mathfrak{Y} \\
&=z^{n+1}+\sum_{k=1}^{\left[\frac{n}{2}\right]}(-1)^{k}\left(\frac{\left(n^{2}-n k+n-k\right)(n-k-1) !}{k(n-2 k+1) !(k-1) !}\right) z^{n+1-2 k}+\mathfrak{Y} \\
&=z^{n+1}+\sum_{k=1}^{\left[\frac{n}{2}\right]}(-1)^{k}\left(\frac{(n+1)(n-k)(n-k-1) !}{k(n-2 k+1) !(k-1) !}\right) z^{n+1-2 k}+\mathfrak{Y} \\
&=z^{n+1}+\sum_{k=1}^{\left[\frac{n}{2}\right]}(-1)^{k} \frac{n+1}{k} \frac{(n-k) !}{(n-k-(k-1)) !(k-1) !} z^{n+1-2 k}+\mathfrak{Y} \\
&=z^{n+1}+\sum_{k=1}^{\left[\frac{n}{2}\right]}(-1)^{k} \frac{n+1}{k}\left(\begin{array}{c}
n-k \\
k-1
\end{array}\right) z^{n+1-2 k}+\mathfrak{Y} \\
&=\sum_{k=0}^{\left[\frac{n}{2}\right]}(-1)^{k} \frac{n+1}{k}\left(\begin{array}{c}
n-k \\
k-1
\end{array}\right) z^{n+1-2 k}+\mathfrak{Y} . \\
& E q
\end{aligned}
$$

Equation 80 .

For any $n \geq 2$, we obtain

$$
z f_{n}(z)-f_{n-1}(z)=\sum_{k=0}^{\left[\frac{n}{2}\right]}(-1)^{k} \frac{n+1}{k}\left(\begin{array}{c}
n-k \\
k-1
\end{array}\right) z^{n+1-2 k}+\mathfrak{Y} .
$$

Equation 81.

Notice that if $n \geq 2$ is even in $\mathbb{N}$, then $\left[\frac{n}{2}\right]=\left[\frac{n+1}{2}\right]$ in $\mathbb{N}$ and hence, the first term of Equation 81 contains the second one. Therefore, one has

$$
n \in 2 \mathbb{N} \Rightarrow z f_{n}(z)-f_{n-1}(z)=\sum_{k=0}^{\left[\frac{n+1}{2}\right]}(-1)^{k} \frac{n+1}{k}\left(\begin{array}{c}
n-k \\
k-1
\end{array}\right) z^{n+1-2 k},
$$

if and only if $z f_{n}(z)-f_{n-1}(z)=f_{n+1}(z)$ for all $n \in 2 \mathbb{N}$. 
Now, assume $n \geq 2$ is odd. Then

$$
\begin{aligned}
& \mathfrak{Y}=(-1)^{\left[\frac{n+1}{2}\right]}\left(\frac{2 n-2}{n+1-2}\right)\left(\frac{\left(n-\left[\frac{n+1}{2}\right]-1\right) !}{\left(n-\left[\frac{n+1}{2}\right]-1-\left[\frac{n+1}{2}\right]+2\right) !\left(\left[\frac{n+1}{2}\right]-2\right) !}\right) z^{n+1-2\left[\frac{n+1}{2}\right]} \\
& =(-1)^{\left[\frac{n+1}{2}\right]}\left(\frac{2 n-2}{n-1}\right)\left(\frac{\left(n-\left[\frac{n+1}{2}\right]-1\right) !}{\left(n-2\left[\frac{n+1}{2}\right]+1\right) !\left(\left[\frac{n+1}{2}\right]-2\right) !}\right) z^{0} \\
& =(-1)^{\left[\frac{n+1}{2}\right]}(2)\left(\frac{\left(n-\left[\frac{n+1}{2}\right]\right) !}{\left(n-2\left[\frac{n+1}{2}\right]+1\right) !\left(n-\left[\frac{n+1}{2}\right]\right)\left(\left[\frac{n+1}{2}\right]-2\right) !}\right) z^{0} \\
& =(-1)^{\left[\frac{n+1}{2}\right]}\left(\frac{n+1}{\left[\frac{n+1}{2}\right]}\right)\left(\frac{\left[\frac{n+1}{2}\right]-1}{n-\left[\frac{n+1}{2}\right]}\right)\left(\begin{array}{c}
n-\left[\frac{n+1}{2}\right] \\
{\left[\frac{n+1}{2}\right]-1}
\end{array}\right) z^{0} \\
& =(-1)^{\left[\frac{n+1}{2}\right]}\left(\frac{n+1}{\left[\frac{n+1}{2}\right]}\right)\left(\frac{n+1-2}{2 n-n-1}\right)\left(\begin{array}{c}
n-\left[\frac{n+1}{2}\right] \\
{\left[\frac{n+1}{2}\right]-1}
\end{array}\right) z^{0} \\
& =\left((-1)^{\left[\frac{n+1}{2}\right]}\left(\frac{n+1}{\left[\frac{n+1}{2}\right]}\right)\left(\begin{array}{c}
n-\left[\frac{n+1}{2}\right] \\
{\left[\frac{n+1}{2}\right]-1}
\end{array}\right)\right) z^{0} \text {. }
\end{aligned}
$$

Therefore

$$
n \in 2 \mathbb{N}+1 \Longrightarrow \mathfrak{Y}=\left((-1)^{\left[\frac{n+1}{2}\right]}\left(\frac{n+1}{\left[\frac{n+1}{2}\right]}\right)\left(\begin{array}{c}
n-\left[\frac{n+1}{2}\right] \\
{\left[\frac{n+1}{2}\right]-1}
\end{array}\right)\right) z^{0}
$$

Equation 84.

So, if $n \geq 2$ is odd then

$$
z f_{n}(z)-f_{n-1}(z)=\sum_{k=0}^{\left[\frac{n+1}{2}\right]}(-1)^{k} \frac{n+1}{k}\left(\begin{array}{c}
n-k \\
k-1
\end{array}\right) z^{n+1-2 k},
$$

if and only if $z f_{n}(z)-f_{n-1}(z)=f_{n+1}(z)$. Therefore, by Equation 82 and Equation 85, one can conclude that

$$
n \geq 2 \in \mathbb{N} \rightarrow z f_{n}(z)-f_{n-1}(z)=f_{n+1}(z) .
$$

Equation 86.

The above result shows that $f_{n+1}\left(\varepsilon_{0}\right)=\varepsilon_{0} f_{n}\left(\varepsilon_{0}\right)-f_{n-1}\left(\varepsilon_{0}\right)$, for $n \geq 2 \in \mathbb{N}$, with $f_{1}\left(\varepsilon_{0}\right)=\varepsilon_{0}$, and $f_{2}\left(\varepsilon_{0}\right)=\varepsilon_{0}^{2}-2$. By Equation 66, one can conclude that if

$$
f_{n}(z)=\sum_{k=0}^{\left[\frac{n}{2}\right]}(-1)^{k}\left(\frac{n}{k}\right)\left(\begin{array}{c}
n-k-1 \\
k-1
\end{array}\right) z^{n-2 k} \text {, then } \mathfrak{X}_{n}=f_{n}\left(\varepsilon_{0}\right) I_{2}
$$

Equation 87.

for all $n \in \mathbb{N}$.

This result shows that the generators $x_{1}$ and $x_{2}$ of $\Gamma_{2}^{2}$ induces the analytic data in $M_{2}(\mathbb{C})$ depending on the elements $x_{1} x_{2}$ and their inverses $x_{2} x_{1}$ up to Equation 87.

Group Algebras $A_{\alpha^{n}, \Gamma_{N}^{2}}$

Let $\Gamma$ be an arbitrary discrete group, and let $(H, \alpha)$ be a Hilbert-space representation of $\Gamma$ consisting of a Hilbert-space $H$ and the group-action $\alpha: \Gamma \rightarrow L(H)$, making $\alpha(g): H \rightarrow H$, for all $g \in \Gamma$ be linear operators (or linear transformations) on $H$, satisfying $\alpha\left(g_{1} g_{2}\right)=\alpha\left(g_{1}\right) \alpha\left(g_{2}\right)$, for all $g_{1}, g_{2} \in \Gamma$, and $\alpha\left(g^{-1}\right)=(\alpha(g))^{-1}$, for all $g \in \Gamma$, where $L(H)$ is the operator algebra consisting of all linear operators on $H$. 
Note that, if we are working with topologies, then one may replace $L(H)$ with $B(H)$, the operator algebra consisting of all bounded (or continuous) linear operators on $H$. However, we are not considering topologies throughout this paper, so we simply set the representational models for $L(H)$ here.

Our group $\Gamma_{N}^{2}$ has its Hilbert-space representations $\left(\mathbb{C}^{n}, \alpha^{n}\right)$, for all $n \in \mathbb{N} \backslash\{1\}$, and each element $g$ of $\Gamma_{N}^{2}$ is realized as $\alpha^{n}(g) \in \mathfrak{M}_{n}^{2}(N)$ in

$$
M_{n}(\mathbb{C})=L\left(\mathbb{C}^{n}\right)=B\left(\mathbb{C}^{n}\right) . \quad \text { Equation } 88 .
$$

The first equality holds because, under finite-dimensionality, all linear operators on $\mathbb{C}^{n}$ are matrices acting on $\mathbb{C}^{n}$, and vice versa. The second equality holds, because, under finite-dimensionality, all pure-algebraic operators on $\mathbb{C}^{n}$ are automatically bounded (and hence, continuous).

For an arbitrary group $\Gamma$, realized by $(H, \alpha)$, one obtains the isomorphic group $\alpha(\Gamma)$ in $L(H)$, i.e., $\Gamma \stackrel{\text { Group }}{=} \alpha(\Gamma) \in L(H)$. So, one can construct a subalgebra,

$$
A_{\alpha, \Gamma}=\mathbb{C}[\alpha(\Gamma)] \text { of } L(H),
$$

Equation 89.

where $\mathbb{C}[X]$ is the polynomial ring over the set $X$. Such rings $\mathbb{C}[X]$ form algebras under polynomial addition and polynomial multiplication over $\mathbb{C}$, and hence, under the inherited operator addition and operator multiplication, $\mathbb{C}[\alpha(\Gamma)]$ forms an algebra in $L(H)$.

Definition 3. Let $\Gamma$ be a group and let $(H, \alpha)$ be a Hilbert-space representation of $\Gamma$. The subalgebra $A_{\alpha, \Gamma}$ of $L(H)$ in the sense of Equation 89 is called the group algebra of $\Gamma$ induced by $(H, \alpha)$.

It is not difficult to show that if

$$
A_{\Gamma}^{o}=\mathbb{C}[\Gamma],
$$

Equation 90.

the pure-algebraic algebra generated by $\Gamma$, as a polynomial ring in $\Gamma$, then

$$
A_{\Gamma}^{o} \stackrel{\mathrm{Alg}}{=} A_{\alpha, \Gamma},
$$

Equation 91.

for all Hilbert-space representations $(H, \alpha)$ of $\Gamma$ algebraically, where “Alg”" means “algebra isomorphic to.”

Proposition 13. Let $\Gamma_{N}^{2}$ be the group defined in Equation 1, and let $\left(\mathbb{C}^{n}, \alpha^{n}\right)$ be our n-dimensional representations of $\Gamma_{N}^{2}$, for all $n \in \mathbb{N} \backslash\{1\}$. Let $\mathfrak{M}_{n}^{2}(N)$ be the $n$-dimensional, order 2 , $N$-generator subgroups of $M_{n}(\mathbb{C})$, for all $n \in \mathbb{N} \backslash\{1\}$. If $A_{\alpha^{n}, \Gamma_{N}^{2}}$ are in the sense of Equation 89, and $A_{\Gamma_{N}^{2}}$ is the group algebra in the sense of Equation 90, then

$$
A_{\Gamma_{N}^{2}} \stackrel{A l g}{=} A_{\alpha^{n}, \Gamma_{N}^{2}}=\mathbb{C}\left[\mathfrak{M}_{n}^{2}(N)\right], \text { for all } n \in \mathbb{N} .
$$

Equation 92.

Proof. The proof is deduced from Equation 91. Recall that $\alpha^{n}\left(\Gamma_{2}^{2}\right)=\mathfrak{M}_{n}^{2}(N)$, for all $n \in \mathbb{N}$.

A Group Algebra $A_{\alpha^{2}, \Gamma_{2}^{2}}$

In this section, we take the group algebra in the sense of Equation 89,

$$
A_{\alpha^{2}, \Gamma_{2}^{2}} \stackrel{\text { denote }}{=} \mathcal{A}_{2}
$$

Equation 93.

as a subalgebra of $M_{2}(\mathbb{C})=L\left(\mathbb{C}^{2}\right)$, under our representation $\left(\mathbb{C}^{2}, \alpha^{2}\right)$. By Equation 92, pure-algebraically, the algebra $\mathcal{A}_{2}$ of is algebra isomorphic to the group algebra $A_{\Gamma_{2}^{2}}=\mathbb{C}\left[\Gamma_{2}^{2}\right]$.

By Equation 51, all elements of $\mathcal{A}_{2}$ are the linear combinations of the matrices formed by

$$
I_{2}, X_{1}, X_{2},\left(X_{1} X_{2}\right)^{n},\left(X_{2} X_{1}\right)^{n} \text {, or }\left(X_{1} X_{2}\right)^{n} X_{1},\left(X_{2} X_{1}\right)^{n} X_{2},
$$

Equation 94. 
for all $n \in \mathbb{N}$, where $X_{j}=\alpha^{2}\left(x_{j}\right)$, for $j=1,2$, since $\mathcal{A}_{2}=\mathbb{C}\left[\mathfrak{M}_{2}^{2}(2)\right] \in M_{2}(\mathbb{C})$.

As we have seen in the previous section, the building blocks of $\mathcal{A}_{2}$ satisfy the following.

(i) $I_{2}, X_{1}, X_{2},\left(X_{1} X_{2}\right)^{n} X_{1}$, and $\left(X_{2} X_{1}\right)^{n} X_{2}$ are self-invertible in $\mathcal{A}_{2} \subset M_{2}(\mathbb{C})$.

(ii) $\left(\left(X_{1} X_{2}\right)^{n}\right)^{-1}=\left(X_{2} X_{1}\right)^{n}$, for all $n \in \mathbb{N}$.

Corollary 14. Let $X_{1}$ and $X_{2}$ be the generating matrices of $\mathfrak{M}_{2}^{2}(2)$, and hence, those of $\mathcal{A}_{2}$. Then

$$
\begin{aligned}
& \left(\left(X_{1} X_{2}\right)^{n}\right)^{-1}=f_{n}\left(\varepsilon_{0}\right) I_{2}-\left(X_{1} X_{2}\right)^{n} \\
& \left(\left(X_{2} X_{1}\right)^{n}\right)^{-1}=f_{n}\left(\varepsilon_{0}\right) I_{2}-\left(X_{2} X_{1}\right)^{n}
\end{aligned}
$$

Equation 95.

for all $n \in \mathbb{N}$, where $\left(f_{n}\right)_{n=2}^{\infty}$ is the functional sequence from Equation 74 .

Proof. By Equation 87, we have $\left(X_{1} X_{2}\right)^{n}+\left(X_{2} X_{1}\right)^{n}=f_{n}\left(\varepsilon_{0}\right) I_{2} \in \mathcal{A}_{2}$, where $f_{n}$ are in the sense of Equation 74, and $\varepsilon_{0}$ is in the sense of Equation 63, for all $n \in \mathbb{N}$. So,

$$
\left(X_{2} X_{1}\right)^{n}=f_{n}\left(\varepsilon_{0}\right) I_{2}-\left(X_{1} X_{2}\right)^{n} \in \mathcal{A}_{2}
$$

Equation 96.

for all $n \in \mathbb{N}$. Thus,

$$
\left(\left(X_{1} X_{2}\right)^{n}\right)^{-1}=f_{n}\left(\varepsilon_{0}\right) I_{2}-\left(X_{1} X_{2}\right)^{n}
$$

Equation 97.

for all $n \in \mathbb{N}$.

Similarly, we obtain $\left(\left(X_{2} X_{1}\right)^{n}\right)^{-1}=f_{n}\left(\varepsilon_{0}\right) I_{2}-\left(X_{2} X_{1}\right)^{n}$, for all $n \in \mathbb{N}$.

It means that if $T$ is a matrix of $\mathcal{A}_{2}$ in the form of either $\left(X_{1} X_{2}\right)^{n}$ or $\left(X_{2} X_{1}\right)^{n}$ for any $n \in \mathbb{N}$, then $T^{-1}=f_{n}\left(\varepsilon_{0}\right) I_{2}-T$.

Proposition 15. Let $A \in \mathfrak{M}_{2}^{2}(2)$ in $\mathcal{A}_{2}$. Then

$$
A^{-1}=\left\{\begin{array}{l}
A \\
r_{A} I_{2}-A
\end{array}\right.
$$

Equation 98.

for some $r_{A} \in \mathbb{C}$.

Proof. If $A \in \mathfrak{M}_{2}^{2}(2)$ in $\mathcal{A}_{2}$, then $T$ is one of the forms listed in Equation 94. As we discussed above, if $A$ is $I_{2}, X_{1}, X_{2}$, $\left(X_{1} X_{2}\right)^{n} X_{1}$, or $\left(X_{2} X_{1}\right)^{n} X_{2}$, for $n \in \mathbb{N}$, then it is self-invertible in $\mathcal{A}_{2}$. i.e., $A^{-1}=A$ in $\mathcal{A}_{2}$.

If $A$ is either $\left(X_{1} X_{2}\right)^{n}$, or $\left(X_{2} X_{1}\right)^{n}$, for any $n \in \mathbb{N}$, then, by Equation 95, there exists $r_{A} \in \mathbb{C}$, such that $A^{-1}=r_{A}-A$ in $\mathcal{A}_{2}$.

The above proposition characterizes the invertibility of $\mathfrak{M}_{2}^{2}(2)$ in $\mathcal{A}_{2}$.

Trace of Certain Matrices of $\mathcal{A}_{2}$

Let $\mathcal{A}_{2}$ be the group algebra of $\Gamma_{2}^{2}$ induced by $\left(\mathbb{C}^{2}, \alpha^{2}\right)$ in $M_{2}(\mathbb{C})$, in the sense of Equation 93. Since the algebra $\mathcal{A}_{2}$ is a subalgebra of $M_{2}(\mathbb{C})$, one can naturally restrict the trace $\operatorname{tr}$ on $M_{2}(\mathbb{C})$ to that on $\mathcal{A}_{2}$. i.e., the pair $\left(\mathcal{A}_{2}, \operatorname{tr}\right)$ forms a noncommutative free probability space. ${ }^{7,8}$

In this section, we are interested in trace of certain types of matrices in $\mathcal{A}_{2}$. Let $X_{1}$ and $X_{2}$ be the generating matrices of $\mathfrak{M}_{2}^{2}(2)$, and hence, those of $\mathcal{A}_{2}$. Define a new element $T$ of $\mathcal{A}_{2}$ by

$$
T=X_{1}+X_{2} \in \mathcal{A}_{2}
$$

Equation 99. 
By the self-invertibility of $X_{1}$ and $X_{2}$, this matrix is understood as the radial operator of $\mathcal{A}_{2} \cdot{ }^{5}$

Observe that

$$
\begin{aligned}
T^{2} & =\left(X_{1}+X_{2}\right)^{2} \\
& =X_{1}^{2}+\left(X_{1} X_{2}+X_{2} X_{1}\right)+X_{2}^{2} \\
& =I_{2}+f_{1}\left(\varepsilon_{0}\right) I_{2}+I_{2} \\
& =\left(f_{1}\left(\varepsilon_{0}\right)+2\right) I_{2} \\
T^{3} & =\left(X_{1}+X_{2}\right)^{3} \\
& =\left(X_{1}+X_{2}\right)^{2}\left(X_{1}+X_{2}\right) \\
& =\left(f_{1}\left(\varepsilon_{0}\right)+2\right) T \\
T^{4} & =T^{3} T \\
& =\left(f_{1}\left(\varepsilon_{0}\right)+2\right) T^{2} \\
& =\left(f_{1}\left(\varepsilon_{0}\right)+2\right)^{2} I_{2} \\
T^{5} & =T^{4} T \\
& =\left(f_{1}\left(\varepsilon_{0}\right)+2\right)^{2} T \\
T^{6} & =T^{5} T \\
& =\left(f_{1}\left(\varepsilon_{0}\right)+2\right)^{2} T^{2} \\
& =\left(f_{1}\left(\varepsilon_{0}\right)+2\right)^{3} I_{2}
\end{aligned}
$$

Equation 100.

where $f_{n}$ are in the sense of Equation 74,

$$
f_{n}(z)=\sum_{k=0}^{\left[\frac{n}{2}\right]}(-1)^{k}\left(\frac{n}{k}\right)\left(\begin{array}{c}
n-k-1 \\
k-1
\end{array}\right) z^{n-2 k},
$$

Equation 101.

for all $n \in \mathbb{N}$, and hence, $f_{1}\left(\varepsilon_{0}\right)=\varepsilon_{0}$. Inductively, one obtains the following.

Theorem 16. Let $T=X_{1}+X_{2}$ be the radial operator of $\mathcal{A}_{2}$, where $X_{1}$ and $X_{2}$ are the generating matrices of $\mathcal{A}_{2}$. Then

$$
\begin{aligned}
T^{2 n} & =\left(\varepsilon_{0}+2\right)^{n} I_{2} \\
T^{2 n+1} & =\left(\varepsilon_{0}+2\right)^{n} T,
\end{aligned}
$$

in $\mathcal{A}_{2}$, for all $n \in \mathbb{N}$, where $\varepsilon_{0}=\operatorname{det}\left(X_{1}\right)+\operatorname{det}\left(X_{2}\right)-\operatorname{det}\left(X_{1}+X_{2}\right)$.

Proof. The proof is inductive and relies on the observations above. Indeed, one can find $T^{2 n}=\left(f_{1}\left(\varepsilon_{0}\right)+2\right)^{n} I_{2}$ and $T^{2 n+1}=\left(f_{1}\left(\varepsilon_{0}\right)+2\right)^{n} T$ in $\mathcal{A}_{2}$ for all $n \in \mathbb{N}$. Finally, since $f_{1}(z)=z$, we obtain $f_{1}\left(\varepsilon_{0}\right)=\varepsilon_{0}$.

Corollary 17. Let $T=n X_{1}+X_{2}$ be the radial operator of $\mathcal{A}_{2}$. Then

$$
\operatorname{tr}\left(T^{n}\right)= \begin{cases}2\left(\varepsilon_{0}+2\right)^{n / 2} & \text { if } n \text { is even } \\ 0 & \text { if } n \text { is odd }\end{cases}
$$

Equation 103.

for $n \in \mathbb{N}$. 
Proof. Set

$$
X_{j}=\left(\begin{array}{cc}
t_{j} & s_{j} \\
\frac{1-t_{j}^{2}}{s_{j}} & -t_{j}
\end{array}\right) \in \mathcal{A}_{2}, \text { for } j=1,2
$$

Equation 104.

where $\left(t_{1}, s_{1}\right)$ and $\left(t_{2}, s_{2}\right)$ are strongly distinct pair in $\mathbb{C}^{\times} \times \mathbb{C}^{\times}$. By Equation 102, if $T$ is given as above in $\mathcal{A}_{2}$, then $T^{2 n}=\left(\varepsilon_{0}+2\right)^{n} I_{2}$, and $T^{2 n+1}=\left(\varepsilon_{0}+2\right)^{n} T$, for $n \in \mathbb{N}$. Thus, it is not difficult to check that

$$
\begin{aligned}
\operatorname{tr}\left(T^{2 n}\right) & =\operatorname{tr}\left(\left(\begin{array}{cc}
\left(\varepsilon_{0}+2\right)^{n} & 0 \\
0 & \left(\varepsilon_{0}+2\right)^{n}
\end{array}\right)\right) \\
& =\left(\varepsilon_{0}+2\right)^{n}+\left(\varepsilon_{0}+2\right)^{n} \\
& =2\left(\varepsilon_{0}+2\right)^{n},
\end{aligned}
$$

Equation 105.

for any $n \in \mathbb{N}$, By the direct computation and by Equation 104, one has

$$
T=\left(\begin{array}{cc}
t_{1}+t_{2} & s_{1}+s_{2} \\
\frac{1-t_{1}^{2}}{s_{1}}+\frac{1-t_{2}^{2}}{s_{2}} & -\left(t_{1}+t_{2}\right) .
\end{array}\right)
$$

Equation 106.

So $\operatorname{tr}(T)=\left(t_{1}+t_{2}\right)+\left(-\left(t_{1}+t_{2}\right)\right)=0$. Also, again by Equation 102, we have

$$
\begin{aligned}
\operatorname{tr}\left(T^{2 n+1}\right) & =\operatorname{tr}\left(\left(\varepsilon_{0}+2\right)^{n} T\right) \\
& =\left(\varepsilon_{0}+2\right)^{n} \operatorname{tr}(T) \\
& =0,
\end{aligned}
$$

Equation 107.

for any $n \in \mathbb{N}$.

\section{APPLICATION: $\mathcal{A}_{2}$ AND LUCAS NUMBERS}

Here, as application of the previous sections, we study connections between analytic data obtained from elements of $\mathcal{A}_{2}$ and Lucas numbers. More details about Lucas numbers are found in the references and cited papers thereof. ${ }^{7-11}$

From the main result, Equation 87, of the previous section, we find a functional sequence $\left(f_{n}\right)_{n=1}^{\infty}$, with its $n$-th entries

$$
f_{n}(z)=n \sum_{k=0}^{\left[\frac{n}{2}\right]}(-1)^{k}\left(\frac{n}{k}\right)\left(\begin{array}{c}
n-k-1 \\
k-1
\end{array}\right) z^{n-2 k} .
$$

Equation 108.

for all $n \in \mathbb{N}$, satisfying $\left(X_{1} X_{2}\right)^{n}+\left(\left(X_{1} X_{2}\right)^{n}\right)^{-1}=f_{n}\left(\varepsilon_{0}\right) I_{2} \in \mathcal{A}_{2}$, where $\varepsilon_{0}=\operatorname{det}\left(X_{1}\right)+\operatorname{det}\left(X_{2}\right)-\operatorname{det}\left(X_{1}+X_{2}\right)$.

For any arbitrarily fixed $n \in \mathbb{N}$, consider the summands

$$
\left(\frac{n}{k}\right)\left(\begin{array}{c}
n-k-1 \\
k-1
\end{array}\right), \text { for } k=0,1, \ldots,\left[\frac{n}{2}\right],
$$

Equation 109.

of $f_{n}(z)$.

Define such quantities of Equation 109 by a form of a function, $g: \mathbb{N} \times \mathbb{N}_{0} \rightarrow \mathbb{C}$ by

$$
g(n, k) \stackrel{\text { def }}{=} \begin{cases}\left(\frac{n}{k}\right)\left(\begin{array}{c}
n-k-1 \\
k-1
\end{array}\right) & \text { if } k=0,1, \ldots,\left[\frac{n}{2}\right] \\
0 & \text { otherwise }\end{cases}
$$

Equation 110.

for all $(n, k) \in \mathbb{N} \times \mathbb{N}_{0}$, where $\mathbb{N}_{0} \stackrel{\text { def }}{=} \mathbb{N} \cup\{0\}$. Note that the definition Equation 110 of the function $g$ represents the (non-alternating parts) of summands of $\left(f_{n}\right)_{n=1}^{\infty}$. 
Theorem 18. Let $g$ be a function from $\mathbb{N} \times \mathbb{N}_{0}$ into $\mathbb{C}$ be as described in Equation 110, Then

$$
g(1,0)=1 \text { and } g(n+1, k)=g(n, k)+g(n-1, k-1),
$$

Equation 111.

for all $(n, k) \in(\mathbb{N} \backslash 1) \times \mathbb{N}$.

Proof. Observe first that:

$$
\begin{aligned}
g(n, k) & =\frac{n}{k}\left(\begin{array}{c}
n-k-1 \\
k-1
\end{array}\right) \\
& =\frac{n}{k} \frac{(n-k-1) !}{(k-1) !(n-k-1-k+1) !} \\
& =\frac{n(n-k-1) !}{(n-2 k) ! k !}
\end{aligned}
$$

Equation 112.

whenever $g(n, k)$ is non-zero, for $(n, k) \in \mathbb{N} \times \mathbb{N}_{0}$.

By Equation 112, we obtain that

$$
g(1,0)=\frac{1(1-0-1) !}{(1-0) ! 0 !}=1
$$

Equation 113.

Consider now that:

$$
\begin{aligned}
g(n, k)+g(n-1, k-1) & =\frac{n}{k}\left(\begin{array}{c}
n-k-1 \\
k-1
\end{array}\right)+\frac{n-1}{k-1}\left(\begin{array}{c}
n-1-(k-1)-1 \\
(k-1)-1
\end{array}\right) \\
& =\frac{n(n-k-1) !}{(n-2 k) ! k !}+\frac{(n-1)(n-k-1) !}{(n-2 k+1) !(k-1) !} \\
& =\frac{n(n-2 k+1)(n-k-1) !+k(n-1)(n-k-1) !}{(n-k k+1) ! k !} \\
& =\frac{\left(n^{2}+n k+n-k\right)(n-k-1) !}{(n-2 k+1) ! k !} \\
& =\frac{(n+1)(n-k) !}{k(2 n-2 k+1)(k-1) !} \\
& =\frac{n+1}{k}\left(\begin{array}{c}
(n+1)-k-1 \\
k-1
\end{array}\right),
\end{aligned}
$$

Equation 114.

and hence,

$$
\begin{aligned}
g(n, k)+g(n-1, k-1) & =\frac{n+1}{k}\left(\begin{array}{c}
(n+1)-k-1 \\
k-1
\end{array}\right) \\
& =g(n+1, k) .
\end{aligned}
$$

Equation 115.

Therefore, one can get the recurrence relation Equation 111, by Equation 113 and Equation 115.

By Equation 111, we can see that the family $\left\{g(n, k):(n, k) \in \mathbb{N} \times \mathbb{N}_{0}\right\}$ relates to the following diagram.

By using the above family $\{g(n, k)\}_{(n, k) \in \mathbb{N} \times \mathbb{N}_{0}}$ of quantities obtained from Equation 110, one can re-write the $n$-th entries $f_{n}$ of Equation 108 as follows;

$$
f_{n}(z)=\sum_{k=0}^{\left[\frac{n}{2}\right]}(-1)^{k}(g(n, k)) z^{n-2 k},
$$

Equation 116. 


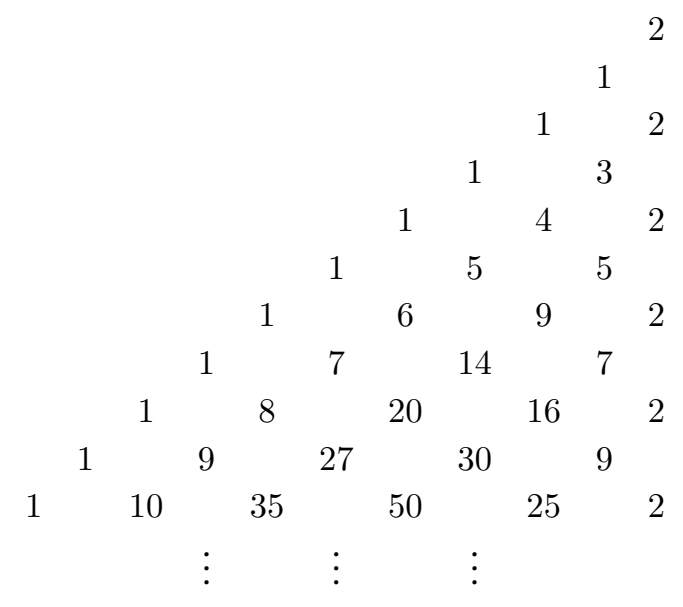

Figure 1. The rows represent $n \in \mathbb{N}$, and the columns represent $k \in \mathbb{N}_{0}$ of the quantities $g(n, k)$. Observe that the diagram is the $L u c a s$ triangle, ${ }^{11}$ induced by the Lucas numbers. ${ }^{7-10}$

for $n \in \mathbb{N}$.

Corollary 19. The coefficients of the functions $f_{n}$ of Equation 108 are determined by the Lucas numbers in the Lucas triangle alternatively.

Moreover, we can verify that

$$
\begin{aligned}
f_{n+1}(z)= & \sum_{k=0}^{\left[\frac{n+1}{2}\right]}(-1)^{k}(g(n+1, k)) z^{n+1-2 k} \\
= & \sum_{k=0}^{\left[\frac{n+1}{2}\right]}(-1)^{k}(g(n, k)+g(n-1, k-1)) z^{n+1-2 k} \\
= & \sum_{k=0}^{\left[\frac{n+1}{2}\right]}(-1)^{k}(g(n, k)) z^{n+1-2 k} \\
& +\sum_{k=1}^{\left[\frac{n+1}{2}\right]}(-1)^{k}(g(n-1, k-1)) z^{n-2 k} \\
= & z\left(\sum_{k=0}^{\left[\frac{n}{2}\right]}(-1)^{k}(g(n, k-1)) z^{n-2 k}\right) \\
& -\sum_{k=0}^{\left[\frac{n-1}{2}\right]}(-1)^{k}(g(n-1, k-1)) z^{n-1-2 k} \\
= & z f_{n}(z)-f_{n-1}(z) .
\end{aligned}
$$

Equation 117.

From the recurrence relation Equation 115, we can re-prove the recurrence relation Equation 78.

Corollary 20. Let

$$
f_{n}(z)=\sum_{k=0}^{\left[\frac{n}{2}\right]}(-1)^{k}(g(n, k)) z^{n-2 k}
$$

Equation 118. 
where $\{g(n, k)\}_{(n, k) \in \mathbb{N} \times \mathbb{N}_{0}}$ are as defined in Equation 110. Then

$$
\begin{aligned}
f_{1}(z) & =z \\
f_{2}(z) & =z^{2}-2 \\
\vdots & \\
f_{n+1}(z) & =z f_{n}(z)-f_{n-1}(z),
\end{aligned}
$$

Equation 119.

for all $n \geq 2$ in $\mathbb{N}$.

Again, the above corollary demonstrates the connection between our Hilbert-space representations of $\Gamma_{2}^{2}$ and Lucas numbers.

\section{REFERENCES}

1. I. Cho, Free Probability on Hecke Algebras and Certain Group $C^{*}$-Algebras Induced by Hecke Algebras, Opuscula Math., (2015) To Appear.

2. I. Cho, Representations and Corresponding Operators Induced by Hecke Algebras, DOI: 10.1007/s11785-014-0418-7, (2014) To Appear.

3. R. Speicher, Combinatorial Theory of the Free Product with Amalgamation and Operator-Valued Free Probability Theory, Momoir, Ame. Math. Soc., 627, (1998)

4. D. Voiculescu, K. J. Dykemma, and A. Nica, Free Random Variables, CRM Monograph Ser., vol.1, ISBN-13: 978-0821811405, (1992)

5. I. Cho, and T. Gillespie, Free Probability on Hecke Algebras, DOI: 10.1007/s11785-014-0403-1, (2014) To Appear.

6. I. Cho, The Moments of Certain Perturbed Operators of the Radial Operator in the Free Group Factors $L\left(F_{N}\right)$, J. Anal. Appl., vol. 5, no.3, (2007) $137-165$.

7. M. Cetin, M. Sezer, and C. Süler, Lucas Polynomial Approach for System of High-Order Linear Differential Equations and Residual Error Estimation, Math. Problems Eng., Article ID: 625984, (2015)

8. A. Nalli, and P. Haukkanem, On Generalized Fibonacci and Lucas Polynomials, Chaos, Solitons \& Fractals, 42, (2009) 3179 - 3186.

9. M. Asci, B. Cekim, and D. Tasci, Generating Matrices for Fibonaci, Lucas and Spectral Orthogonal Polynomials with Algorithms, Internat. J. Pure Appl. Math., 34, no. 2, (2007) 267 - 278.

10. J. Wang, Some New Results for the $(p, q)$-Fibonacci and Lucas Polynomicals, Adv. Diff. Equ., 64, (2014)

11. A. T. Benjamin, The Lucas Triangle Recounted, available at http://www.math.hmc.edu/ benjamin/papers/LucasTriangle.pdf.

\section{ABOUT THE STUDENT AUTHOR}

Ryan Golden is a senior at St. Ambrose University double majoring in Mathematics and Biology with minors in Computer Science and Chemistry. He plans to pursue a PhD in Applied Mathematics, specifically Mathematical and Computational Neuroscience. While his main interests are in the applications of mathematics, he is passionate about theoretical mathematics as well.

\section{PRESS SUMMARY}

In this paper, the authors studied matricial representations of certain finitely presented groups generated by $N$-generators of order-2. As an application, they considered corresponding group algebras under representation. Specifically, they characterized the inverses of all group elements in terms of matrices in the group algebra. From the study of this characterization, the authors realized there are close relations between the trace of the radial operator of the algebras and the Lucas numbers appearing in the Lucas triangle. 


\title{
Evolution of Leucyl-tRNA Synthetase Through Eukaryotic Speciation
}

\author{
Katelyn E. Unvert', Frank A. Kovacs, Chi Zhang, Rachel A. Hellmann-Whitaker and Katelin N. Arndt** \\ ${ }^{a}$ Department of Biology, University of Nebraska, Kearney \\ ${ }^{b}$ School of Biological Sciences, University of Nebraska, Lincoln \\ 'Department of Chemistry and Biochemistry, University of Arizona \\ Students:arndtkn@lopers.unk.edu*,unvertke@lopers.unk.edu \\ Contributing authors: rhang.chi@unl.edu,kovacsfa@unk.edu \\ Mentor: hellmann@email.arizona.edu
}

\begin{abstract}
Aminoacyl-tRNA synthetases (aRSs) are part of the cellular translation machinery and as such, they are essential enzymes for every known cell. Due to their ubiquitous nature, their evolutionary history has been intensely researched to better understand the origins of life on a molecular level. Herein, we examine the evolutionary relatedness of leucyl-tRNA synthetases (LeuRS) from each major eukaryotic branch through the speciation process. This research effort was centered on amino acid sequence data as well as generating homology protein models for each LeuRS enzyme. Comparative analysis of this sequence and structural data for LeuRS amongst eukaryotes has indicated a high level of conservation within the active sites of these enzymes. Phylogenetic analysis confirmed this high degree of conservation as well as established evolutionary relatedness between these LeuRS enzymes. Based on this data, vertical gene transfer propagated LeuRS throughout the eukaryotic domain. Horizontal gene transfer and domain acquisition events were not observed within the eukaryotic organisms studied. Our data also highlighted LeuRS adaptation through the speciation process due to slight variability of scaffolding residues outside of the active site regions. We hypothesize that this variability may be due to mechanistic differences amongst LeuRS enzymes that have assumed nontranslational functionality through the evolutionary process.
\end{abstract}

\section{KEYWORDS}

tRNA Synthetase; Leucyl-tRNA Synthetase; Eukaryotic Evolution; LeuRS Conservation; Vertical Gene Transfer; Horizontal Gene Transfer; Convergent Evolution; Primordial Enzymes

\section{INTRODUCTION}

Aminoacyl-tRNA synthetases (aaRSs) are the evolutionary link between an ancient RNA-based world and the contemporary amino acid-driven world. ${ }^{1}$ Hypotheses developed from substantiated data has determined that aaRSs are an ancient family of enzymes that displayed greater fitness for aminoacylation catalysis, thus displacing ribozymes from this catalytic niche. ${ }^{\mathbf{1 - 2}}$ As such, aaRSs chemically link nucleic acids and amino acids through the aminoacylation reaction, which plays a central role in translation. ${ }^{3}$ The aminoacylation reaction consists of aaRSs binding to their cognate amino acid as well as tRNA to generate a hybrid aminoacylated tRNA structure. ${ }^{4}$ Thus, aaRSs are ubiquitous throughout nature and as such their evolutionary origins have been the subject of numerous research studies., 5-9

It is thought that aaRSs evolved to better adapt an existing genetic code to a changing biological environment, in which the kinetic efficiency of the aminoacylation reaction needed to improve to meet the needs of a developing primordial cell or cellular community. ${ }^{7-8}$ The intense evolutionary pressure that existed in the early stages of the emergence of life resulted in evolutionary convergence within the aaRS family of enzymes. ${ }^{1}$ This family of enzymes was thus subdivided into class I and class II aaRSs, with the result: one aaRS per amino acid. ${ }^{10-11}$ Interestingly, class I and class II aaRSs are structurally dissimilar, however, this family of enzymes almost evenly divided with approximately 10 aaRSs in each subgroup. ${ }^{10-13}$ This suggests that a level of evolutionary coordination existed between these two classes of aaRSs.

Given that the family of aaRSs is comprised of approximately 20 enzymes and that there is high catalytic fidelity displayed by each enzyme, the evolutionary path for each class of aaRS necessitated structural divergence from two separate ancient aaRS primordial 
enzymes. ${ }^{5}$ Divergence and propagation of each aaRS subclass may have been precipitated through horizontal gene transfer (HGT), gene duplication, and domain acquisition events. ${ }^{7-9}$ Evidence of HGT and domain acquisition events within the family of aaRSs have been used to explain the complicated phylogeny of these enzymes, since they do not seem to adhere to the canonical organismal phylogeny proposed by Carl Woese. 5 , 7, 14-16 Thus, the molecular evolution of aaRSs is a complex story, in which the details are continuing to unfold.

Class I aaRSs are multi-domain enzymes, with the main domain harboring the aminoacylation active site, with the core catalytic site being comprised of a Rossmann nucleotide binding fold. ${ }^{17-18}$ The Rossmann fold binds to cognate amino acid and tRNA as well as ATP to catalyze the aminoacylation reaction. ${ }^{10}$ Several of the class I aaRSs have a secondary editing domain that is an insertion into the Rossmann fold and is thought to be a later evolutionary addition to these enzymes. ${ }^{16,19}$ In contrast to class I aaRSs, the class II aaRS active site is comprised of antiparallel $\beta$-strands, which are flanked by $\alpha$-helices. ${ }^{20}$

The leucyl-tRNA synthetase (LeuRS) enzyme is a class I aaRS that is thought to have diverged from a common ancestral aaRS that also gave rise to isoleucyl-tRNA synthetase (IleRS) and valyl-tRNA synthetase (ValRS). ${ }^{16},{ }^{21}$ This divergence event is thought to have occurred before the time of the last universal common ancestor (LUCA). ${ }^{9}$ Originally, it was thought that the LeuRS molecular phylogeny was one of the only examples of aaRSs that followed Carl Woese's Archaea-Bacteria-Eukaryota trichotomy. ${ }^{14}$ However, other research evidence suggests that the LeuRS evolutionary history may have been subject to HGT events, as is the case for other aaRSs. ${ }^{7-9}$ These HGT events complicate the molecular phylogeny of LeuRS, but may help explain its ubiquitous nature as well as its biochemical conservation through the speciation process of all three domains of life.

In this study, we analyzed the sequence and structural features of LeuRS enzymes through the eukaryotic lineage to establish evolutionary relatedness. Though we focused our research efforts on LeuRS divergence through the speciation of eukaryotes, we started our comparative analysis with a bacterial example of LeuRS since it is thought that the Archeae and Eukaryota branches split from the bacterial branch. ${ }^{22}$ Highlighting LeuRS evolution through eukaryotic speciation, starting from a bacterial example, shed light on LeuRS propagation throughout the eukaryotic domain of life. Additionally, these studies demonstrate the possibility of pharmacological cross-reactivity potential amongst this group of LeuRS enzymes.

\section{METHODS AND PROCEDURES}

Sequence Comparisons

Multiple sequence alignments were conducted with amino acid sequences for LeuRS enzymes from the following organisms with the corresponding National Center for Biotechnology Information (NCBI) protein identification numbers: Escherichia coli (BAA35289.1), Saccharomyces cerevisiae (EDN60985.1), Arabidopsis thaliana (NP_172433.2), Draosophila melanogaster (AGV77169.1), Danio rerio (XP_698279.6), Xenopus laevis (NP_001087393.1), Anolis carolinensis (XP_003224146.1), Taeniopygia guttata (XP_002189937.1), Mus musculus (NP_598898.2), and Homo sapiens (NP_064502.9). Retrieval of these protein sequences was achieved with the Basic Local Alignment Search Tool (BLAST). ${ }^{23}$ These sequences were then subjected to various multiple sequence alignment analyses using Clustal Omega and PRALINE. ${ }^{24-25}$ The PRALINE sequence alignment results were used to determine relative conservation between LeuRS enzymes, which was in turn used in determining LeuRS structural comparisons.

An additional multiple sequence alignment was conducted using tRNA Leu sequence data from the following sources: Escherichia coli tRNA ${ }^{\text {Leu }}$ sequences with the anticodons: CAA, CAG, GAG, TAA and TAG; Saccharomyces cerevisiae tRNA ${ }^{\text {Leu }}$ sequences with the anticodons: TAA, CAA and TAG; Homo sapiens tRNA ${ }^{\text {Leu }}$ sequences with the anticodons: TAA, AAG and TAG. These tRNALeu sequences were retrieved from the tRNA Genomic Database. ${ }^{26}$ The subsequent multiple sequence alignment analysis was conducted using the T-Coffee alignment tool. ${ }^{27}$

Homology Protein Modeling and Structural Comparisons

To generate the LeuRS homology models, in silico protein structure prediction techniques were utilized. The development and refinement of these LeuRS computational models, including fold recognition methodologies, were conducted using SPARKX and I-TASSER software. ${ }^{28-30}$

The I-TASSER platform is commonly used to generate 3D models of a target protein sequence. It utilizes a composite method that involves amino acid threading, which is guided by sequence alignment and secondary structure prediction paired with various 
structural refinement techniques. Each final homology model is evaluated via a confidence score (C-score), which is based on the threading template alignment quality as well as the convergence of structures coming from the assembly simulations. The $\mathrm{C}$ scores are usually in the range of -5 to 2 with values above -1.5 considered to be significantly more reliable. ${ }^{30}$ Additionally, the models are evaluated by a TM-score, which measures structure similarity of the best threading alignment with the average of all the alignments generated. Models with TM-scores above 0.5 are considered topologically reliable. ${ }^{30}$ Please refer to Table $\mathbf{1}$ for a complete listing of these reliability scores.

\begin{tabular}{|l|l|l|}
\hline \multicolumn{1}{|c|}{ Organism } & C-score & \multicolumn{1}{c|}{ TM-score } \\
\hline Arabidopsis thaliana & 0.44 & $0.77+/-0.1$ \\
\hline Draosophila melanogaster & -1.18 & $0.57+/-0.15$ \\
\hline Xenopus laevis & -1.51 & $0.53+/-0.15$ \\
\hline Homo sapiens & -1.57 & $0.52+/-0.15$ \\
\hline Mus musculus & -1.55 & $0.52+/-0.15$ \\
\hline Saccharomyces cerevisiae & 0.56 & $0.79+/-0.09$ \\
\hline Taeniopygia guttata & -1.29 & $0.55+/-0.15$ \\
\hline Danio rerio & -1.11 & $0.58+/-0.14$ \\
\hline Anolis carolinensis & -1.33 & $0.52+/-0.14$ \\
\hline
\end{tabular}

Table 1. I-TASSER Reliability Scores for all Homology models.

Once the protein homology models were generated, various structural comparisons were made through their superimposition. This was accomplished through the Chimera software suite. ${ }^{31-33}$ Additional structural manipulations, coloring changes, protein fragmentation as well as rotations were also done using the Chimera software suite.

\section{Phylogenetic Tree Development}

Determination of LeuRS amino acid sequence conservation as well as evolutionary relatedness was confirmed with the development of a phylogenetic tree. This phylogenetic tree was generated using the following LeuRS amino acid sequences: Escherichia coli (BAA35289.1), Saccharomyces cerevisiae (EDN60985.1), Arabidopsis thaliana (NP_172433.2), Draosophila melanogaster (AGV77169.1), Danio rerio (XP_698279.6), Xenopus laevis (NP_001087393.1), Anolis carolinensis (XP_003224146.1), Taeniopygia guttata (XP_002189937.1), Mus musculus (NP_598898.2), and Homo sapiens (NP_064502.9). The phyloT: a tree generator software program was utilized to compile this sequence information into a final phylogenic tree. ${ }^{34-35}$

\section{RESULTS}

The process of modern translation of the genetic code is facilitated by an ancient family of enzymes called aminoacyl-tRNA synthetases (aaRSs). ${ }^{36}$ In vitro evolution experiments of ribozymes with aminoacylation activity, suggests that as the ancient RNAbased world evolved, aaRSs may have been one of the first enzymes to develop and displace this ribozyme catalytic niche.1-2 As such, it has been hypothesized that the genetic code pre-dates the existence of aaRSs, and due to evolutionary pressure to improve the catalytic efficiency of the aminoacylation reaction, amino acid-based primordial aaRSs evolved. ${ }^{7}$ These ancestral aaRSs structurally diverged into a family of 20 aaRS enzymes: one for each canonical amino acid. ${ }^{7}$ This structural divergence is largely attributed to horizontal gene transfer (HGT) and domain acquisition events that predate the time of the last universal common ancestor (LUCA). ${ }^{7}{ }^{9}$ Further structural divergence of aaRSs occurred as the speciation process gave rise to the three domains of life. ${ }^{7}$ Interestingly, aaRS domain arrangement and their basic catalytic features have remained relatively unchanged since the time of LUCA. ${ }^{9}$ The ancient origins of a particular aaRS, leucyl-tRNA synthetase (LeuRS), has been well characterized. ${ }^{8}$ This research effort focuses on the structural divergence of LeuRS through the evolution of the eukaryotic lineage starting from bacteria.

The LeuRS enzyme consists of two main catalytic centers, housed within the aminoacylation domain and the connective polypeptide 1 (CP1) domain (Fig. 1). ${ }^{19}, 37$ The aminoacylation domain active site binds to leucine, ATP and cognate tRNALeu to facilitate the aminoacylation reaction. ${ }^{3,} 38$ The CP1 domain active site hydrolyzes misaminoacylated products that are generated by the aminoacylation reaction. ${ }^{39}$ The LeuRS aminoacylation domain is hypothesized to be the most ancient proteinaceous functional unit of LeuRS, evolving from a common ancestor aaRS that structurally diverged into LeuRS, IleRS and ValRS. ${ }^{16,21}$ Using 
BLAST, Clustal Omega and PRALINE multiple sequence alignment tools, we generated numerous sequence alignments of the catalytic core of the LeuRS aminoacylation domain from Escherichia coli (E. coli), Saccharomyces cerevisiae (S. cerevisiae), Arabidopsis thaliana (A. thaliana), Draosophila melanogaster (D. melanogaster), Danio rerio (D. rerio), Xenopus laevis (X. laevis), Anolis carolinensis (A. carolinensis), Taeniopygia guttata (T. guttata), Mus musculus (M. musculus) and Homo sapiens (H. sapiens)(Fig. 2A). This sequence alignment focuses on the signature sequences HIGH and KMSKS from the Rossmann nucleotide binding motif, which is the catalytic core of the LeuRS aminoacylation domain. ${ }^{3}$ The sequence alignment of all the organisms in Figure $2 \mathbf{A}$ indicates that there is considerable conservation within these signature sequences, underscoring their essential function as well as their ancient nature. The PRALINE sequence alignment tool uses a scoring scheme based on conservation, with 0 being the least conserved and 10 being the most conserved. This numeric gradient is paired with a color gradient where 0 is dark blue and 10 is red. As seen in Figure 2A, all the positions within the HIGH and KMSKS signature sequences have conservation values of 6 or greater, excluding E. coli LeuRS. Due to E. coli LeuRS being significantly smaller than the eukaryotic LeuRS enzymes and algorithmic limitations of this sequence alignment tool, the E. coli KMSKS region was not included in this sequence alignment. However, the E. coli LeuRS KMSKS region can be visualized in Figure 2B. Figure $2 \mathbf{B}$ highlights the extreme conservation of the aminoacylation active site within this diverse group of organisms, in which LeuRS homology protein structures were predicted and the structure of the HIGH and KMSKS regions from each organism were superimposed. Based on these results, and given the fact that these organisms span approximately 3.4 billion years of evolutionary time, it is remarkable that these signature sequences and their corresponding structures have remained largely unchanged. ${ }^{40}$ This supports the hypothesis that LeuRSs' catalytic core domain originated prior to the time of LUCA and remained largely unchanged over billions of years. ${ }^{8}$ Thus, vertical gene transfer events predominated the propagation of LeuRS throughout the eukaryotic domain.

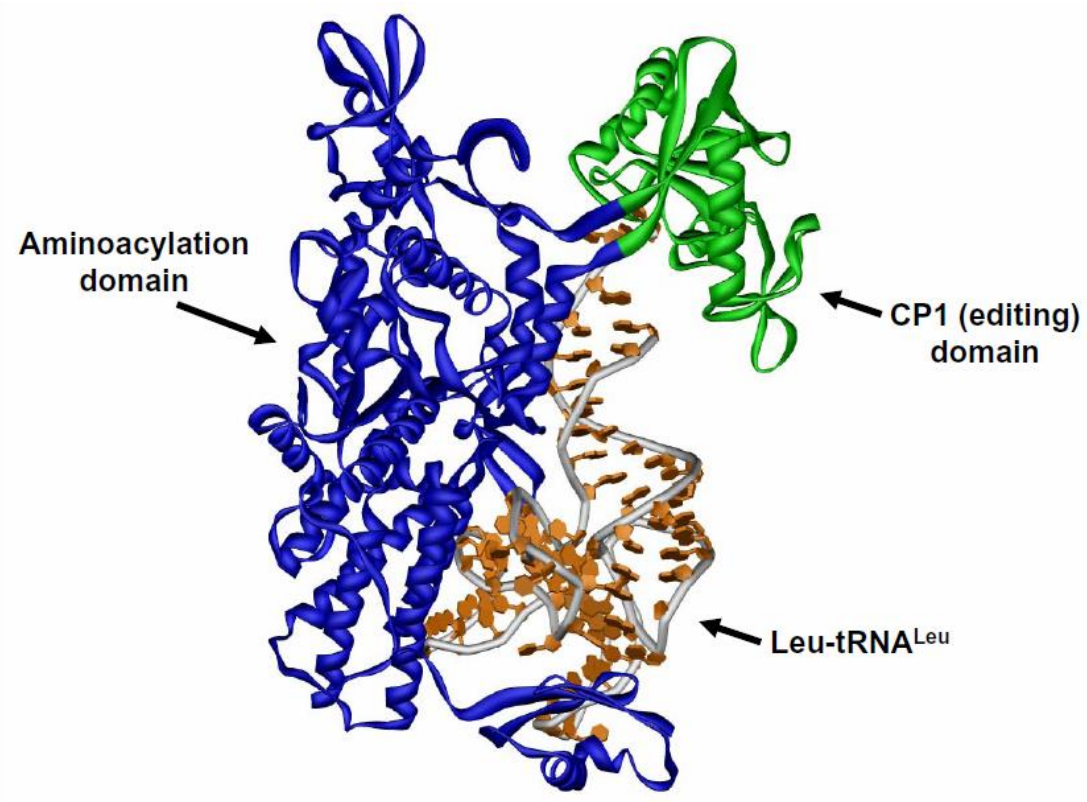

Figure $1 .^{37} \mathrm{X}$-ray crystallography structure of E. coli LeuRS. The E. coli LeuRS crystal structure while in the editing conformation. The aminoacylation domain is blue and the CP1 editing domain is green. The bound Leu-tRNA Leu is highlighted in orange. 


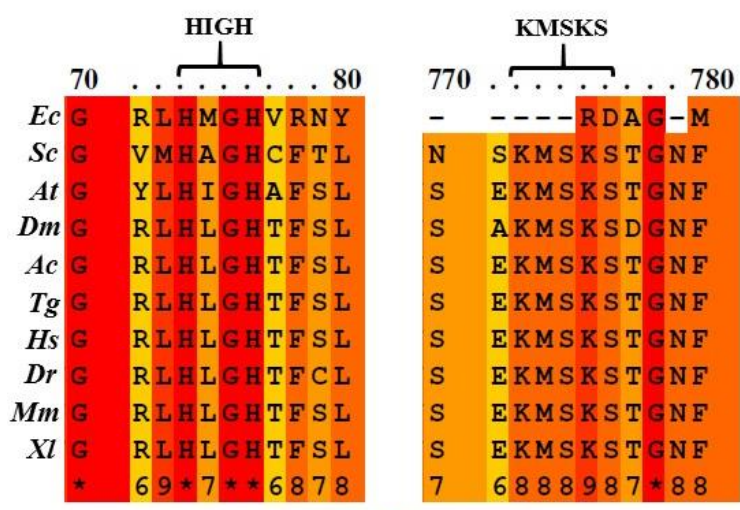

Unconserved 012345678910 Conserved

Figure 2A.

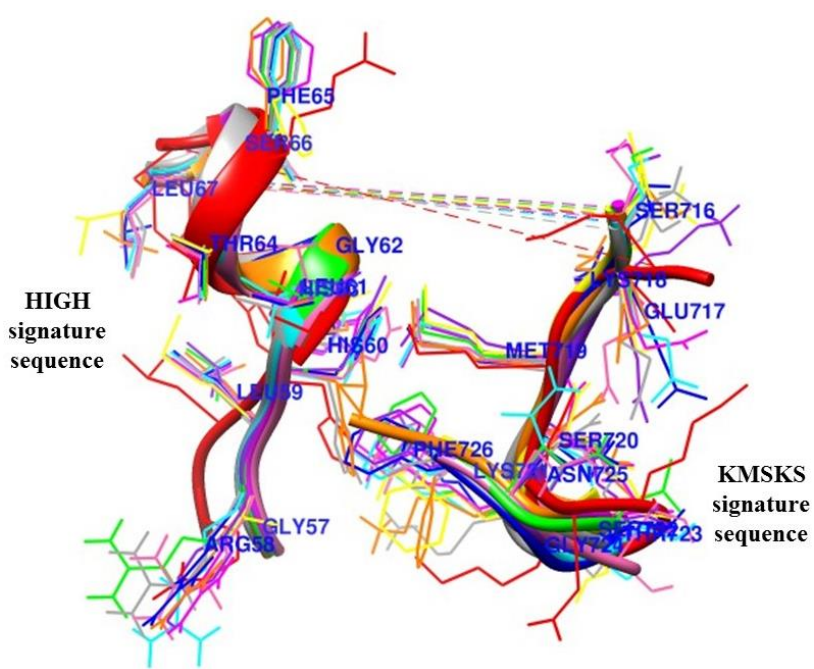

Figure 2B.

Figure 2. Multiple sequence alignment (2A.) and superposition (2B.) of the Aminoacylation active site "HIGH" and "KMSKS" signature sequence regions. 2A. ${ }^{3}$ Brackets above the multiple sequence alignment highlight the "HIGH" and "KMSKS" signature sequences, which are universal sequences found within the LeuRS active site. The signature sequences bind and stabilize ATP and the adenylate intermediate.

2B. ${ }^{37}$ The E. coli LeuRS X-ray crystallography structure is red. The E. coli LeuRS crystal structure is overlaid with the following computationally generated LeuRS homology models. Organism abbreviations and color labels are as follows: Eschericbia coli (Ec), Saccharomyces cerevisiae (Sc) (yellow), Arabidopsis thaliana (At) (purple), Draosophila melanogaster (Dm) (green), Danio rerio (Dr) (orange), Xenopus laevis (Xl) (blue), Anolis carolinensis (Ac) (magenta), Taeniopygia guttata (Tg) (cyan), Mus musculus $(\mathrm{Mm})$ (gray) and Homo sapiens (Hs) (pink).

Additional support for this hypothesis is displayed in the superimposed structures of the entire catalytic core docking site for ATP, leucine and the 3' acceptor stem end of tRNA Leu from E. coli and S. cerevisiae (Fig. 3), which indicates a high level of preservation through eukaryotic evolution. The E. coli LeuRS structure is red and the S. cerevisiae LeuRS structure is yellow. Given that these organisms are thought to be separated by 1.5 billion years of evolutionary time and are structurally dissimilar in many other ways (refer to Fig. 7), it is remarkable the that active sites of these two enzymes are extremely similar. ${ }^{40}$ This result provides evidence that the LeuRS active site originated from a common ancestral aaRS and has virtually remained unchanged. Additionally, these results support the assertion that the root of the three domains of life be placed along the bacterial branch with the Archaea and Eukaryota branches being clustered closely together. ${ }^{22,41}$

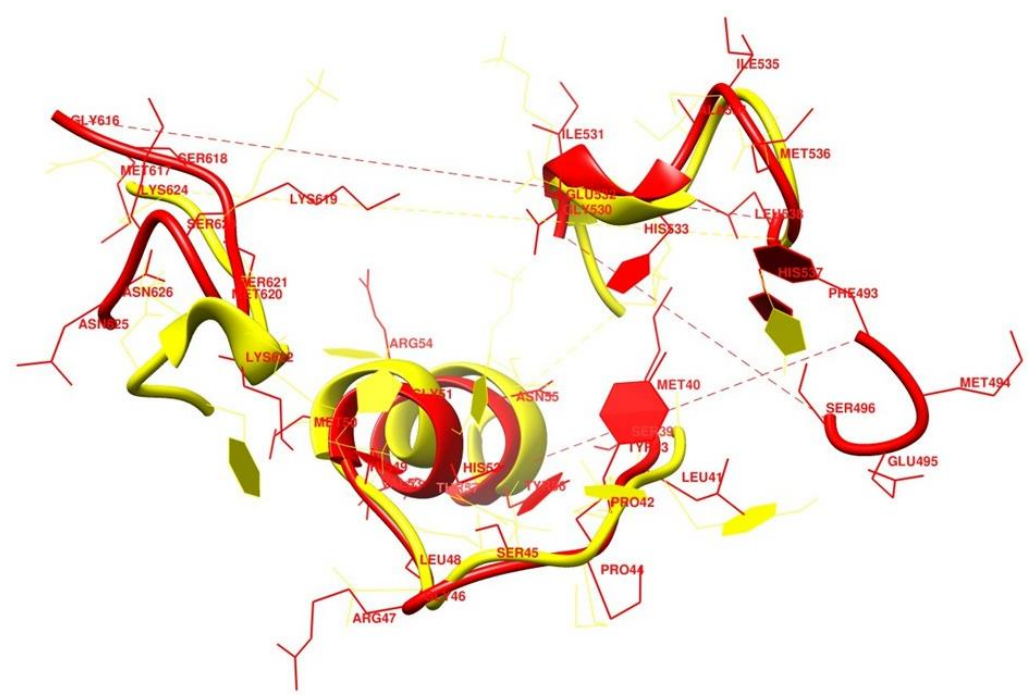

Figure 3. ${ }^{37}$ Aminoacylation active site superimposition of E. coli and S. cerevisiae. Superimposition of the entire aminoacylation active site. The E. coli LeuRS X-ray crystallography structure is red and the $S$. cerevisiae LeuRS homology model is yellow. 


\begin{tabular}{|c|c|c|c|c|c|}
\hline Escherichia_col & N I A Y M K N Q L K & ML GFGYDWSR & $\mathrm{E} \perp \mathrm{A} T \mathrm{~T}$ T $--\mathrm{PE}$ & Y Y RWEQKF F T & E L Y K K G L VYK \\
\hline Saccharomyces_cF & F P P L E S DCT & S L GAR I DWRR & SFVTTDANPY & Y DAF I RWQMN & K L KA A GK I KF \\
\hline Arabidopsis_tha & F P P L AVED L R & A Y G L G CDWRR & SFVTTDVNPF & FDAFVRWQMR & K L K S MGKIVK \\
\hline Drosophila_mela & F P P L A VQDLK & RIGVHVDWRR & TF I T T A N P & FDSFVRWQFN & H I KERGKIMY \\
\hline Anolis_caroline & F P P L A I Q D L K & SMGLKIDWRR & SFVTTDVNPY & YDSFVRWQFL & T LRERNKIKF \\
\hline Taeniopygia_gut & F P P L A VQDLK & SMGLKVDWRR & SF I T T NVNPY & YDSFVRWQFL & T LKERNKIKF \\
\hline Homo_sapiens & F P P L A I QD L K & RMGLKVDWRR & SF I T TDVNPY & YDSFVRWQFL & T L RERNKIKF \\
\hline Danio_rerio & F P P LAVEDLK & RMGLKVDWRR & SF I T T D N P F & YDSFVRWQFI & N L K E R K K I K F \\
\hline Mus_musculus & F P P L A V QD L K & TIGLKVDWRR & SFITTDVNPY & YDSFVRWQFL & T L RERNKIKF \\
\hline Xenopus_laevis & F P P LAVEDLK & SMGLKVDWRR & SF I T T DNPF & YDSFVKWQFV & K I KERNKIKF \\
\hline \multirow[t]{2}{*}{ Consistency } & 8888766888 & $56 * 757 * * 8$ * & $888 * 8768$ * 7 & 8878888874 & 58966 \\
\hline & . . 260 & 270 & 280 & 290 & \\
\hline richia_col & KT SAVNWCPN & DQTVLANEQV & I D & KVERKEIPQW & FIKITAYADE \\
\hline es_c & GERYTIYSEK & $D G Q A C M D H D R$ & ------ & $--\mathrm{EGVT}-\mathrm{PQE}$ & Y I GVKI EALE \\
\hline is_tha & DRRYTIFSPL & $D G Q P C A D H D R$ & A T & $--E G V Q-P Q E$ & Y T L I KME- - \\
\hline La_mela & GKRYTIYSPK & $D G Q P C M D H D R$ & s s & $--E G V G-P Q E$ & Y T L I KMK- - - \\
\hline ine & I Y S P K & DGQPCMDHDR & QT $\mathrm{C}$ & $--E G V G-P Q E$ & Y T L I KMK- - - \\
\hline a_gut & G KRY T I Y S P K & DGQP CMDHDR & ------ & $--E G V G-P Q E$ & Y T L I KMK- - - \\
\hline Homo_sapiens & GKRYTIYSPK & $D G Q P C M D H D R$ & ------ & $--E G V G-P Q E$ & Y T L L K L K - - - \\
\hline rerio & GKRYT I Y SPK & DGQPCMDHDR & ------ & $--E G V G-P Q E$ & Y T L I KMK- - - \\
\hline sculus & GKRYTIYSPK & $D G Q P C M D H D R$ & QT & $--\mathrm{EGVG}-\mathrm{PQE}$ & Y T LVKLK- - - \\
\hline aevis & GKRYTIYSPR & DGQPCMDHDR & Q T G- - - - - - & $--\mathrm{EGVG}-\mathrm{PQE}$ & Y T L I KMK- - - \\
\hline \multirow[t]{2}{*}{ Consistency } & 7788888886 & * 887879888 & $67 * 0000000$ & $00 * 8850 * * 8$ & 9769877000 \\
\hline & $\ldots 310$ & $\ldots \ldots 320$ & 330 & 34 & \\
\hline chia_col & $L L N D L D K L D H$ & WPDTVKTMQR & NW I GRSEGVE & ITFNVNDYDN & TLTVYTTRP \\
\hline nyces_c & $F$ A D D A $--\mathrm{A} K I$ & I DSSSDLDKS & K K F------- & ---------- & YFVAATLRPE \\
\hline dopsis_tha & $----v--V K P$ & F P L K L GP LEG & KRV ------- & ---------- & F L A A A T L R P E \\
\hline Dros & $----V--L E-$ & VPKALSSI-K & Q P I - - - - - & ---------- & F MVAATLRPE \\
\hline Anol & $----v--V E P$ & Y PVKLSGLKG & KN I- - - - - - & ---------- & F LVA A T LRPE \\
\hline Taer & $=----V--L D P$ & YPAKLSGLRG & KN I - - - - - - & ---------- & F LVA A T L R P E \\
\hline sapiens & $----V--L E P$ & YPSKLSGLKG & K N I & ---------- & F LVA A T L R E \\
\hline Dan & $----V--V E P$ & YPTKLGALKG & K T V & ------ & F LVAATLRPE \\
\hline usculus & $----V--L E P$ & YPSKLSGLKG & K N I & ------ & FLVAATLRPE \\
\hline Xenopus_laevis & $----V--L E P$ & LPTKLSGLKG & RNV ------- & ---------- & FLVAATLRP \\
\hline \multirow[t]{2}{*}{ Consistency } & 0000800775 & 5846764766 & 7480000000 & 0000000000 & $78898 * 8 * * 9$ \\
\hline & $\ldots 360$ & $\ldots \ldots \ldots$ & $\ldots \ldots \ldots 380$ & & \\
\hline Escl & TFMGCTYLAV & A A G H P LAQK- & $-----\mathrm{AAENN}$ & PELAAF IDEC & I A E AE- \\
\hline Sac & $=T M Y G Q T C C F V$ & SP T I EYG IFD & A G- - D SYFIT & TERAFKNMSY & P K R GF Y \\
\hline Ara & TMYGQTNAWV & L P DGKYGAYE & I S-ETEVF I L & TERAALNLAY & NPQE P \\
\hline nela & TMYGQTNCW L & H P D I KY I A W $Q$ & ANKNNEVWVS & TRRAARNMTY & AVEGEI \\
\hline Anol & TMF GQTNCWV & RPDMKY I GFE & TI-NGDIFIC & TQRAARNMSY & QGFTKINGVV \\
\hline pygia_gut & TMFGQTNCWL & RPDMKYIGFE & T G-GGDIFIC & SQRAARNMSY & QGF TRDNGVL \\
\hline apiens & TMF GQTNCWV & RPDMKY I GFE & TV-NGDIFIC & TQKAARNMSY & QGFTKDNGVV \\
\hline Danio_rerio & TMF GQTNCW I & RPDMKYVVFE & TA-NGDLFIS & TQRSARNMSY & ENGVV \\
\hline Mus & TMF GQTNCWV & RPDMKY I GFE & TA-NGDIFIC & TQRAARNMSY & Q GF TKHNGVV \\
\hline Xenopus_laevis & TMF GQTNCW L & RP DMPYIAFE & T T-NGDIFIC & TQRAARNMSY & DNGVV \\
\hline \multirow[t]{2}{*}{ Consistency } & * 87 * 8 * 6778 & 5875685466 & 5304466784 & 7779868778 & 9677535754 \\
\hline & $\ldots \ldots$ & 420 & .430 & & \\
\hline Esch & MATMEKKG-V & DTGF KAVHPL & TGEE I PVWAA & $N--F V L M E Y G$ & TGAVMAVPGH \\
\hline s_c & C K P I V T V P G KA & F I G T K I HAPQ & SVYPE-LRIL & PMETVIATKG & T GVVTCVPSN \\
\hline tha & SCLVELTGYD & L I G L P L R S P L & SVNEI-IYAL & P MLT I L TNKG & TGIVTSVPSD \\
\hline Drosophila_mela & KV LAEVTGQD & L L GVP L SAPL & TKH KV -VYS L & PMLSIKEDKG & T GVVTSVPSD \\
\hline Anolis_caroline & PVVKELMGED & I L GAP L SAPI & TSYKV-IYTL & PMLTIKEDKG & T GVVTSVPSD \\
\hline Taeniopygia_gut & PVVKELMGEE & I LGAALSAPL & TNYKV-IYAL & PMLTIKEDKG & TGVVTSVPSD \\
\hline Homo_sapiens & PVVKELMGEE & I LGASISAPL & TSYKV-IYVL & PMLT IKEDKG & T GVVTSVPSD \\
\hline Danio_rerio & PVIMNIMGQD & I LGCALSAPL & TSYKT-IYAL & PMLTIKEDKG & TGVVTSVPSD \\
\hline usculus & PVVKELMGEE & I LGASISAPL & TCYKV-VYVL & PMLTIKEDKG & TGVVTSVPSD \\
\hline Xenopus_la & PVVKELMGED & LLGAALSAPL & TSYKV-IYAL & PMLTIKEDKG & TGVVTSVPSD \\
\hline \multirow[t]{2}{*}{ Consistency } & 5675775 *55 & $67 * 54867 * 8$ & 8467708768 & 886796678 * & $\star \star 8 * 87 * \star 87$ \\
\hline & $\ldots \ldots \ldots 460$ & $\ldots \ldots \ldots 470$ & $\ldots \ldots \ldots 480$ & $\ldots \ldots \ldots 490$ & $\ldots \ldots \ldots 50$ \\
\hline $\operatorname{col}$ & DQRDYEFASK & ---------- & Y GL ------- & - N I K PVIL- - & ---------- \\
\hline & S P D D Y I T KD & L L H K P E - - Y & Y G I K P E W I D H & - E IVP IMHTE & KY GDL T A KA I \\
\hline that & A P D D Y MALQD & L I KK P A L QDK & Y GVKTEW L P T & - E I I P I I N I P & EFGDKA A E KV \\
\hline a_melas & SPDDYAAIVD & I QKKEAFRQK & $Y G L K D E M V L P$ & Y E P I P I EV P & TLGKLSAVHA \\
\hline Anolis_caroline & SPDDIAALRD & L KKKQ P F RAK & Y G I KDD MV L P & F E P V P V IEIP & GYGQLSAPMI \\
\hline Taeniopygia_gut & SPDDFAALRD & L K K K Q A L R VK & Y G I RDEMVLP & F E P VP I IE I P & GYGS LCAPFV \\
\hline Homo_sapiens & SPDDIAAIRD & I K KK $Q$ A L RAK & Y G I RDD MV L P & FE PVPVIEIP & GFGN L SAVTI \\
\hline Danio_rerio & A P D D I A A L RD & I K KKQ A L REK & Y G I QD F M V L P & F E P V P I I E I P & GYGN L S A P LV \\
\hline Mus_musculus & SP D D LA A L RD & L K K K Q A L R T K & F G I RDD MV L P & F E P V PVLEIP & G I GN L P A V TV \\
\hline & A P D D I A A L RD & L K K KQ A L RQK & Y G I KDEMV L P & F E P V P I I D I P & GYGN L S A P A V \\
\hline Consistency & 788 * 567768 & 7468554526 & $9 * 86555745$ & $4858 * 98566$ & 4584648326 \\
\hline
\end{tabular}

\section{Unconserved 12345678910 Conserved}

Figure 4. ${ }^{39}$ Multiple sequence alignment of the CP1 Editing Domain. The black box on the multiple sequence alignment highlights the "threonine-rich" region. The threonine-rich region provides chemical specificity to the CP1 domain hydrolytic active site. Organism are as follows: Escherichia coli, Saccharomyces cerevisiae, Arabidopsis thaliana, Draosopbila melanogaster, Danio rerio, Xenopus laevis, Anolis carolinensis, Taeniopygia guttata, Mus musculus, and Homo sapiens. 


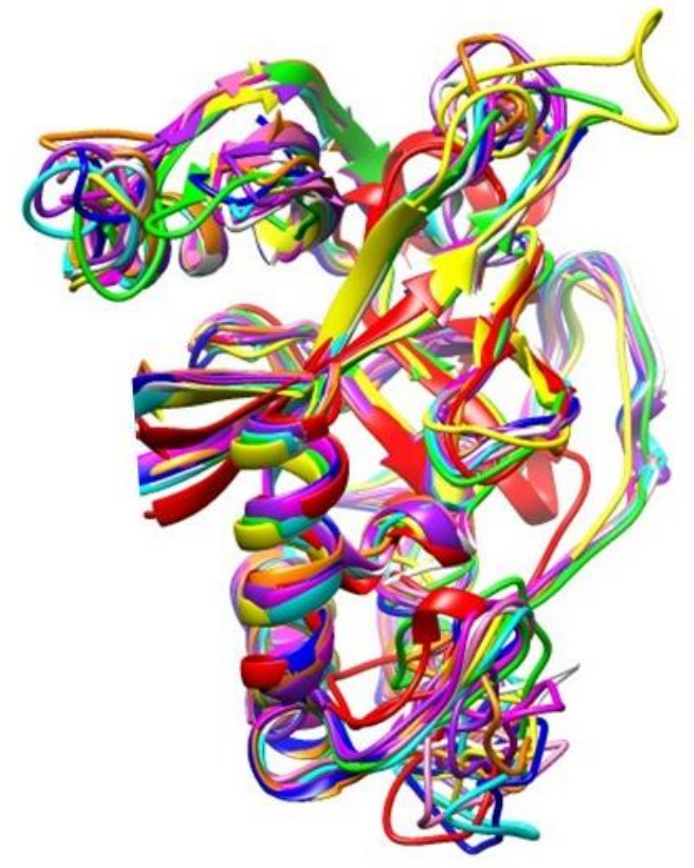

Figure 5A.

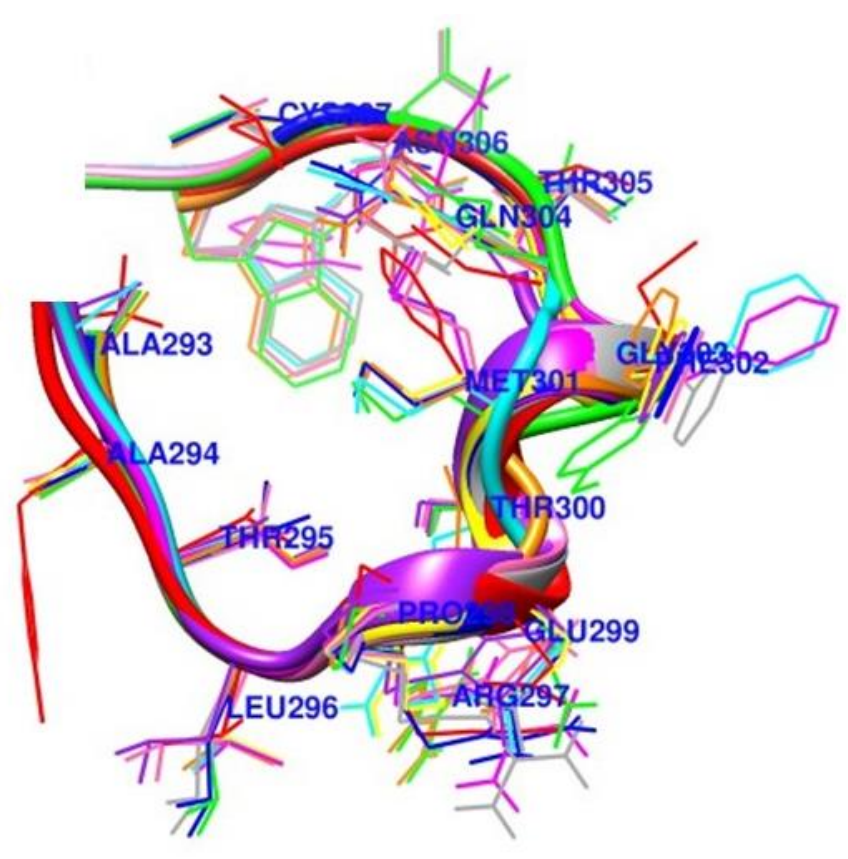

Figure 5B.

Figure 5. CP1 Domain superimposition. ${ }^{37,39}$ 5A. Superimposition of the entire CP1 domain. 5B. Superimposition of the "threonine-rich" region within the hydrolytic active site of the CP1 domain. The E. coli LeuRS X-ray crystallography structure is red. The E. coli LeuRS crystal structure is overlaid with the following computationally generated LeuRS homology models: S. cerevisiae (yellow), A. thaliana (purple), D. melanogaster (green), D. rerio (orange), X. laevis (blue), A. carolinensis (magenta), T. guttate (cyan), M. musculus (gray), and H. sapiens (pink).

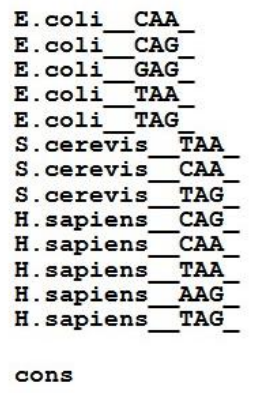

BAD AVG GOOD

GCCGAAGTGGCGAAATCGGTAGACGCAGTTGATTCAAAATCAACCGTAG--AAATACGTGCCGGTT CGAGTCCGGCCTTCGGCACCA GCGAAGGTGGCGGATTGGTAGACGCGCTAGCT TCAGGTGTTAGTGTCCTTACGGACGTGGGGTT CAAGTCCCCCCCTCGCACCA GCCGAGGTGGTGGAATTGGTAGACACGCTACCT TGAGGTGGTAGTGCCCAATAGGGCTTACGGGTT CAAGTCCCGTCCTCGGTACCA GCCCGGATGGTGGAATCGGTAGACACAAGGGAT TTAAAATCCCTCGGCGTTCGCGCTGTGCGGGTT CAAGTCCCGCTCCGGGTACCA

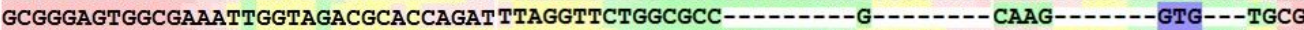
GGAGGGTTGGCCGAGT-GGTA-AGGCGGCAGACTTAAGATCTGTTGGACGTTTGTCCGCGCGAGTT CGAACCTCGCA---TCCTTCA GGTTGTTTGGCCGAGC-GGTA-AGGCGCCTGAT TCAAGCTCAGGTAT-C-GTAAGATGCAAGAGTTCGAATCTCTTA---GCAACCA GGGAGTTTGGCCGAGT-GGTA-AGGCGTCAGAT TTAGGCTCTGATAT-C-TTCGGATGCAAGGGTTCGAATCCCTTA---GCTCTCA GGGAGTTTGGCCGAGT-GGTA-AGGCGTCAGAT TTAGGCTCTGATAT-C-TTCGGATGCAAGGGTTCGAATCCCTTA----GCTCTCA GTCAGGATGGCCGAGT-GGTA-AGGCGCCAGAC TCAAGTTCTGGTCTCCGGATGGAGGCGTGGGTT CGAATCCCACT---TCTGACA GTTAAGATGGCAGAGC-CGCAATTGCATAAAAC TTAAAAC------T-T--TACAA--CAGAGGTT CAACTCCTCTT---CTTAACA GGGCCAGTGGCTCAAT-GGAA-ATGCGTCTGACTAAGAATCAG--------AAGAT--CCAGCCT TGACTCCTGGC---TGGCTCA GGTAGTGTGGTTGAAT-GGTA-AGGCACTGAAT TTAGGCTCCAGTCT-C-TTTGGGGACGTGGGTT TAAATCCCACT---GCTGCAA

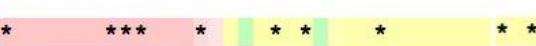

Figure 6. Multiple sequence alignment of tRNA ${ }^{\text {Leu }}$ Isotypes. The multiple sequence alignment of tRNA Leu from E. coli, S. cerevisiae, and $H$. sapiens shows sequence variability amongst these different isotopic forms. The tRNA ${ }^{\text {Leu }}$ isotypes of CAA, CAG, GAG, TAA, TAG, and AAG originate from E. coli, S. cerevisiae, and $H$.

sapiens. These three organisms were chosen because they represent the greatest evolutionary time lapse amongst organisms on the eukaryotic branch.

LeuRS domain insertions are thought to be later evolutionary additions to LeuRS, to improve catalytic efficiency. ${ }^{16,21,42}$ Indeed, the auxiliary LeuRS domains like the CP1 domain and the ZN-1 domain have been biochemically analyzed and shown to drastically improve LeuRS catalysis. ${ }^{39}, 43$ The CP1 domain is a unique insertion into the Rossmann fold and serves to hydrolyze misaminoacylated products. ${ }^{39}$ The CP1 domain is crucial to maintain LeuRS function and fidelity due to the structurally promiscuous nature of the aminoacylation active site, which can readily accommodate structural homologs of leucine. ${ }^{44}$ The structural ambiguity of the aminoacylation active site would be a natural evolutionary consequence since LeuRS, IleRS and ValRS all structurally diverged from a common aaRS ancestor and the cognate amino acids that these aaRSs charge, namely leucine, isoleucine and valine, are all structurally similar. ${ }^{16,21,42}$ Therefore, the CP1 domain functions as an editing domain that allows the LeuRS enzyme to distinguish between leucine and other amino acid structural homologs. To determine the extent of conservation within the CP1 domain, sequence alignment data was gathered for the entire sequences of the CP1 domains from E. coli, $S$. 
cerevisiae, A. thaliana, D. melanogaster, D. rerio, X. laevis, A. carolinensis, T. guttata, M. musculus and H. sapiens (Fig. 4). From this data, it is evident that there is significant sequence conservation within the CP1 domains for these organisms, with the one outlier being E. coli. This finding may be a computational artifact due to E. coli's CP1 domain being generally smaller than the CP1 domains from the eukaryotic sources. Based on the sequence alignment, the CP1 domains from E. coli and S. cerevisiae had 31\% identity and $48 \%$ homology. The CP1 domains from $S$. cerevisiae and $A$. thaliana share 45\% identity and 64\% homology. The CP1 domains from $A$. thaliana and D. melanogaster share $56 \%$ identity and $73 \%$ homology. The CP1 domains from D. melanogaster and $D$. rerio share 64\% identity and 81\% homology. The CP1 domains from D. rerio and X. laevis share $84 \%$ identity and $92 \%$ homology. The CP1 domains from $X$. laevis and $A$. carolinensis share $87 \%$ identity and $93 \%$ homology. The CP1 domains from $A$. carolinensis and $T$. guttata share 88\% identity and 94\% homology. The CP1 domains from T. guttata and M. musculus share 85\% identity and 94\% homology. The CP1 domains from M. musculus and H. sapiens share 93\% identity and 94\% homology. Therefore, to build upon these results, structural superimpositions of the entire CP1 domain as well as the hydrolytic active site were generated (Fig. 5A and 5B). Computational models were generated for all CP1 domains to provide consistent analysis, which were based off existing crystal structures of LeuRS. From Figure 5A, there is considerable structural conservation within all the CP1 domain structures. Some structural variation is observed within the loop regions of the CP1 domain. This finding is expected since these disorganized protein structural features are difficult to computationally model. Therefore, these slight structural perturbations may be a computational artifact. An alternative explanation could be that some of these loop regions could interact with tRNA ${ }^{L e u}$, helping to facilitate its translocation between the active sites of LeuRS. ${ }^{45}$ Since there is variability amongst tRNA ${ }^{\text {Leu }}$ sequences across species lines (Fig. 6), a reasonable assumption would be that regions within the CP1 domain that interact and bind with tRNA ${ }^{\text {Leu }}$ would also have variability.

Close examination of the CP1 domain hydrolytic active site pocket in Figure 5B indicates that there is considerable structural conservation. Indeed, amongst the eukaryotic species the hydrolytic active site sequences that comprise the threonine-rich region are completely conserved. ${ }^{39}$ When compared to the E. coli threonine-rich region, there are only two amino acids that deviate from the eukaryotic sequences, the T248 and D251 residues. A similar result was found by Pang. et. al. through an amino acid sequence comparison of the threonine-rich region from dissimilar organisms such as E. coli and H. sapiens. ${ }^{46}$ Evolutionarily, this would indicate that the CP1 domain would have evolved early-on as LeuRS structurally diverged from its common ancestral aaRS to provide chemical specificity for the accurate incorporation of leucine during protein synthesis. Thus, based on these results, we hypothesize that the CP1 domain would have probably been the first leucine-specific domain adaptation, which allowed a more catalytically efficient LeuRS to replace an existing ribozyme for leucine aminoacylation catalysis.

In addition to examining how the aminoacylation and CP1 domain active sites evolved, other sequence and structural features were studied to gain a more global perspective on how LeuRS evolved through the speciation of eukaryotes. Therefore, a qualitative evolutionary tree was generated to visually follow the molecular evolution of LeuRS structure through the speciation of eukaryotes starting from a bacterial example (Fig. 7). Each node represents a major split between taxa within the eukaryotic domain of life. ${ }^{40}$ Since it is thought that the eukaryotic domain of life is rooted from the bacterial domain, the E. coli LeuRS structure was used as the initial point of reference. ${ }^{22,41}$ Hence, the E. coli LeuRS structure is entirely gray, any subsequent changes within the LeuRS structure are highlighted in red. Structural deviations for each species were determined through generating a sequence alignment of the LeuRS from the organism of interest and the LeuRS from the organism on the next lower branch. These pairwise sequence alignments were generated using the PRALINE sequence alignment tool. Residues with a conservation score of "4" or less were highlighted in red. Therefore, the S. cerevisiae LeuRS structural deviation from E. coli LeuRS was determined through a sequence alignment of $E$. coli LeuRS and $S$. cerevisiae LeuRS. In like manner, the $A$. thaliana LeuRS structural deviation from $S$. cerevisiae LeuRS was determined through a sequence alignment of $S$. cerevisiae LeuRS and $A$. thaliana LeuRS. This same pattern of sequence comparison for each LeuRS was used to determine LeuRS structural deviations throughout the evolutionary tree. 


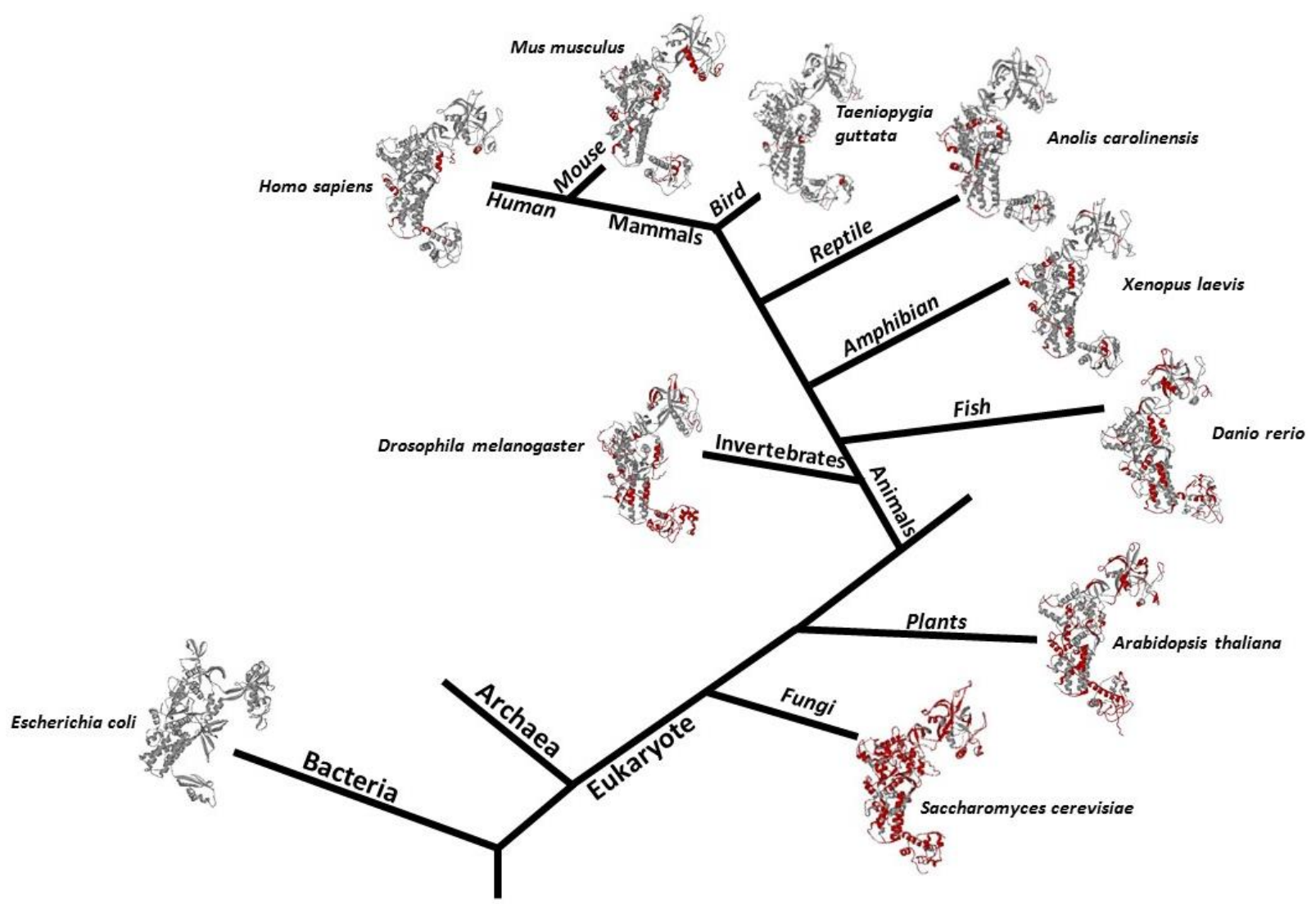

Figure 7. Evolutionary changes of LeuRS Among Eukaryotes. This qualitative phylogenetic tree compares LeuRS structures from each taxon within the eukaryotic domain of life, starting from a bacterial example. Universal features of LeuRS are gray and regions that have been subject to evolutionary change are red. Since the eukaryotic domain is thought to have arisen from bacterial origins, the E. coli LeuRS crystal structure is entirely gray ${ }^{22,37}$.

Figure 7 shows that there is significant structural deviation in the initial comparison between E. coli LeuRS and S. cerevisiae LeuRS. In part, this structural deviation is due to the size difference between these two enzymes. The S. cerevisiae LeuRS enzyme is 230 amino acids larger than the E. coli LeuRS enzyme. The size difference between these two enzymes as well as the other eukaryotic LeuRSs highlighted in this study could be due to additional non-translational catalytic functionality that was assumed by eukaryotic LeuRSs through the evolutionary process. ${ }^{47}$ Emerging evidence indicates that many aaRSs within eukaryotes have evolved critical roles that maintain cellular function outside of their canonical role in translation. ${ }^{47}$ Specifically, eukaryotic LeuRSs are known to participate in the TOR metabolic pathway, which functions to maintain amino acid metabolism. . $^{4-49}$

The observed sequence and structural deviations seem to occur within scaffolding residues. As has been previously stated, the aminoacylation active site and CP1 domain hydrolytic active site are highly conserved, thus structural deviation was not observed within these regions. The highest frequency of sequence and structural changes were observed between the lower-order eukaryotic species, namely; $S$. cerevisiae, A. thaliana, D. melanogaster and D. rerio. These sequence and structural differences may be due to mechanistic adaptation within the catalytic functionality of these enzymes. For example, it has been reported that $S$. cerevisiae LeuRS has an altered mechanistic behavior within the TOR metabolic pathway when compared to that of H. sapiens LeuRS mechanistic behavior within the same pathway. ${ }^{48-50}$ Mechanistic adaptation would be a natural consequence of the evolutionary process, especially since these enzymes function in different cellular environments with different extracellular ques. When considering the high frequency of sequence and structural changes within the lower-order eukaryotes, it is important to consider that this set of organisms differs greatly in their overall biology. The diversity within the lower-order eukaryotes can most simply be stated through general comparisons between the organisms, such as: single-cellular vs. multi-cellular; photosynthetic vs. non-photosynthetic; terrestrial vs. aquatic. Diversity within these biological systems would necessitate evolutionary adaptation, giving rise to LeuRS enzymes that have altered mechanistic function and potentially a different repertoire of molecular binding partners within each of these cell types. To further support this assertion, close examination of the LeuRS structures originating 
from higher-order organisms along the animal branch, namely; X. laevis, A. carolinensis, T. guttata, M. musculus, and H. sapiens, have very little sequence and structural deviation. Again, a general biological comparison of these organisms could be used to explain these results. This group of animals are multicellular, terrestrial and share many of the same physiological processes, albeit with some obvious differences. In short, these organisms are more biologically related than the lower-order eukaryotes. Within the higher-order eukaryotes, subgroup comparisons of LeuRSs shows extreme conservation. These subgroups are $A$. carolinensis : $T$. guttata and M. musculus: H. sapiens. This finding is not surprising given that lizards and birds are close evolutionary relatives. ${ }^{51}$ The evolutionary relationship between mice and humans is even more closely linked, as they are both mammals and are separated by only 96 million years of evolutionary time: less than half of the evolutionary time that separates lizards and birds.51-53

The evolutionary relationships between eukaryotic LeuRS enzymes that have been reported herein were further verified through the generation of a quantitative molecular phylogenetic tree based on LeuRS amino acid sequences (Fig. 8). This phylogenetic tree was developed through the PhyloT software program and verified through sequence analysis for the evolutionary hierarchy established with the Figure 7 results. The phylogenetic tree indicates that E. coli LeuRS and S. cerevisiae LeuRS are more closely related to each other when compared to the other lower-order eukaryotic organisms. From $A$. thaliana LeuRS to X. leavis LeuRS there is step-wise evolutionary relatedness for each of the branch points. This degree of evolutionary connectivity between the lower-order organisms has been previously explained for the Figure 7 results. In like manner, the subgroupings of $A$. carolinensis : T. guttata and M. musculus: H. sapiens LeuRS enzymes within this molecular phylogenetic tree have also been explained in the context of Figure 7 results. Therefore, the molecular phylogenetic tree confirms the Figure 7 results and associated hypotheses.

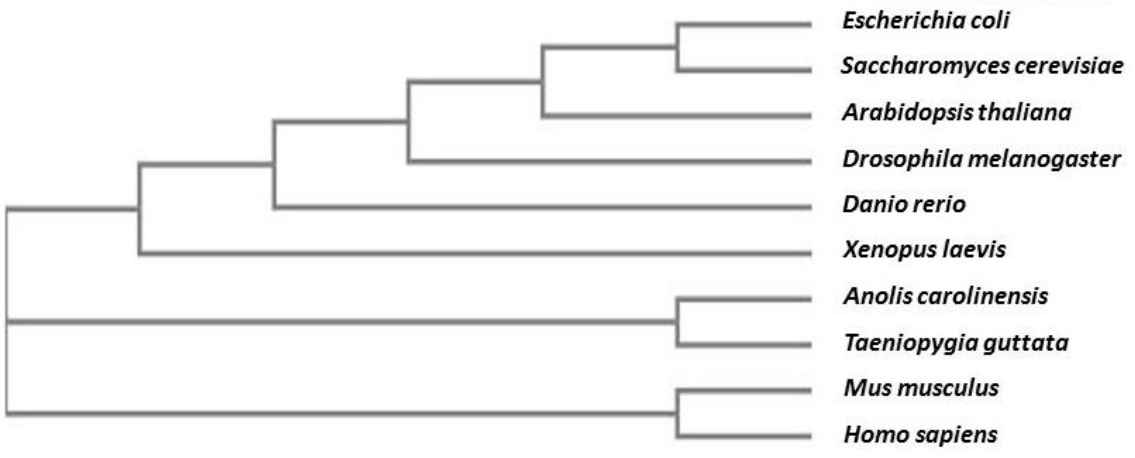

Figure 8. Molecular Phylogenetic Tree of Eukaryotic LeuRS. ${ }^{22}$ This phylogenetic tree compares LeuRS amino acid sequences from each taxon within the eukaryotic domain of life, starting from a bacterial example. Since the eukaryotic domain is thought to have arisen from bacterial origins, the E. coli LeuRS sequence is included.

Lastly, each of the LeuRS homology structures were superimposed on their nearest evolutionary relative using the Chimera software program. Therefore, E. coli LeuRS and S. cerevisiae LeuRS were superimposed on one another (Fig. 9A). This superimposition provided an essential structural comparison of these two LeuRS enzymes. Again, E. coli LeuRS is in red and $S$. cerevisiae LeuRS is in yellow. From this structural overlap, the greatest differences are within the CP1 and C-terminal domains. These differences are largely related to the overall size of these domains. Given that $S$. cerevisiae LeuRS has adopted additional non-translational functionality, these size differences could support these non-translational catalytic and binding functions. hese LeuRS structural differences may be due to sequence differences within the individual tRNA ${ }^{\text {Leu }}$ for each organism, which subsequently affects the tRNA ${ }^{\mathrm{Leu}}$ structures (Fig. 6).

The superimposition of $A$. thaliana LeuRS (purple) and D. melanogaster LeuRS (green) (Fig. 9B) as well as the superimposition of D. rerio LeuRS (orange) and X. laevis LeuRS (blue) (Fig. 9C) shows some structural differences largely within the CP1 and Cterminal domains. Again, these differences may be due to the very different biological environments in which these LeuRS enzymes operate, which would give rise to mechanistic differences in the catalytic functions of these enzymes. The superimposition of $A$. carolinensis, LeuRS (magenta) and T. guttate LeuRS (cyan) indicates that these enzymes are almost identical, albeit with some slight changes in a secondary structural elemental feature within the aminoacylation domain for T. guttate LeuRS, which may be an artifact of the protein modeling process (Fig. 9D). The fact that these two enzymes are structurally very similar is indicative of their close evolutionary relatedness, as previously explained. Lastly, the superimposition of the $M$. musculus LeuRS (gray) and H. sapiens LeuRS (pink) enzymes shows that they are virtually identical (Fig. 9E). This was an expected result given that 
these enzymes have mammalian origins, which are separated by very little evolutionary time. Thus, the superimposition of these LeuRS structures spanning the eukaryotic lineage supports our previous evolutionary assertions as well as provides the data necessary to visually compare how LeuRS changed or was conserved through evolutionary time.

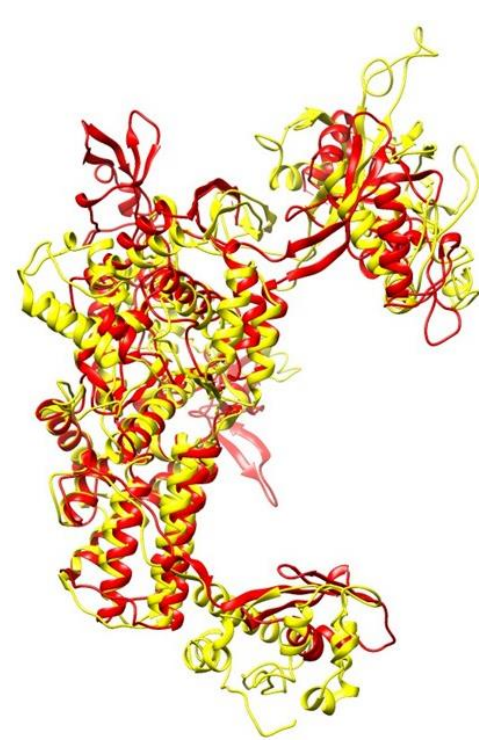

Figure 9A.

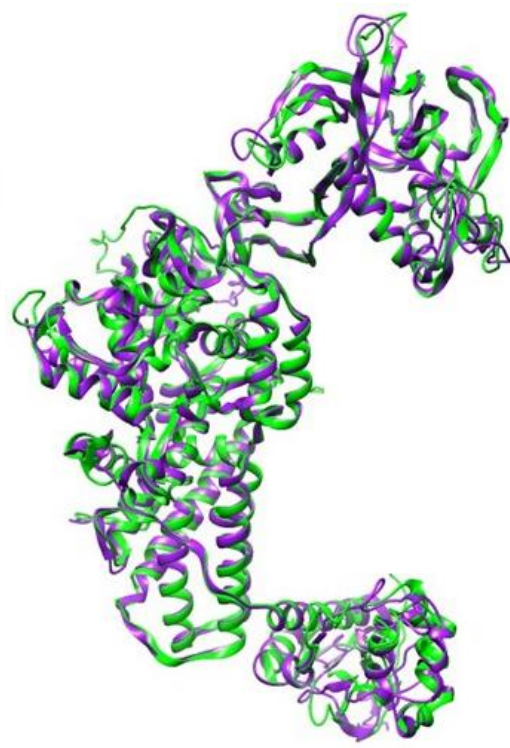

Figure 9B.

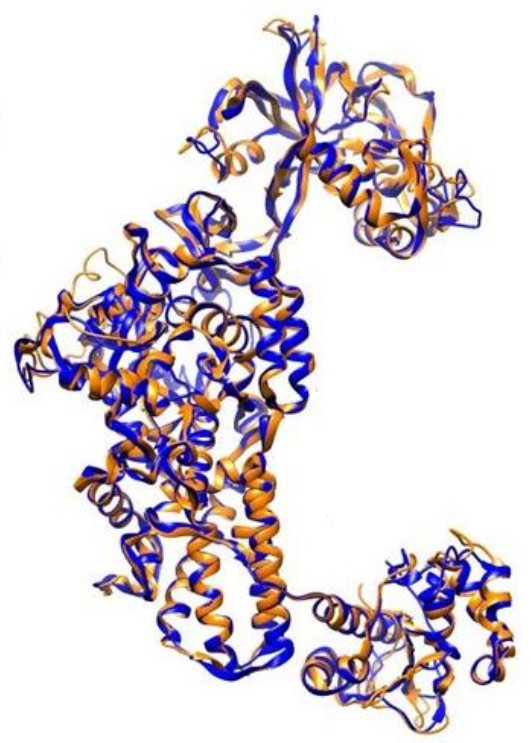

Figure 9C.

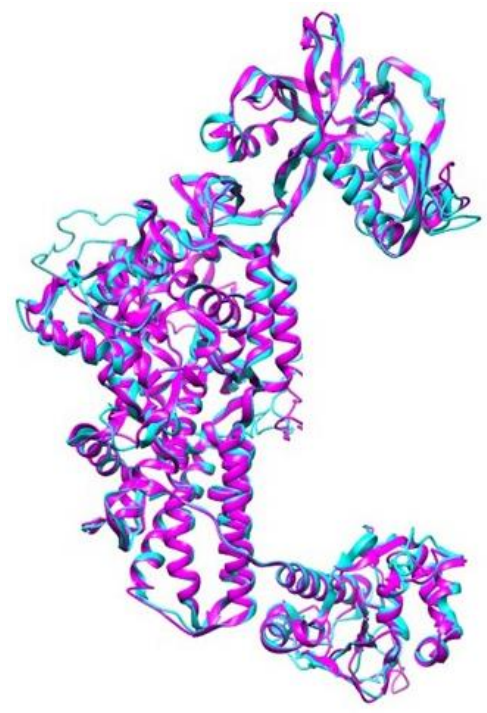

Figure 9D.

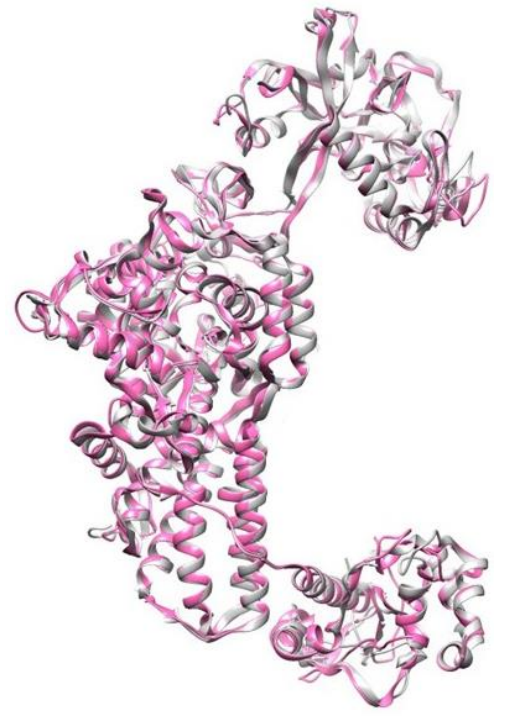

Figure 9E.

Figure 9. ${ }^{37}$ Superimposition of the LeuRS enzyme structures to compare and contrast structural changes amongst eukaryotic organisms.

9A. The E. coli LeuRS X-ray crystallography structure is red, overlaid with the S. cerevisiae LeuRS homology model in yellow.

9B. The A. thaliana LeuRS homology model (purple) is overlaid with the D. melanogaster LeuRS homology model (green). 9C. The D. rerio LeuRS homology model (orange) is overlaid with the X. laevis LeuRS homology model (blue).

9D. The A. carolinensis LeuRS homology model (magenta) is overlaid with the T. guttate LeuRS homology model (cyan). 9E. The M. musculus LeuRS homology model (gray) is overlaid with the H. sapiens LeuRS homology model (pink). 


\section{DISCUSSION}

The evolutionary origins of aaRSs have been well characterized by numerous research studies focused on understanding the development of the aminoacylation domain and aaRS catalytic specificity. 1, 5, 7-9, 21 Consensus among these research efforts is that aaRS enzymes have ancient origins that predate LUCA. To build upon these previously reported findings, this research effort aimed to understand the more contemporary evolutionary history of LeuRS, specifically through the eukaryotic lineage. The results reported herein indicate that the aminoacylation active site as well as the CP1 domain active site are both highly conserved. Indeed, a comparison of these active sites across domain and species lines indicates that there is very little variation within the sequences or structures of LeuRSs from these different species. This was a surprising result given that there are billions of years of evolutionary history separating the species highlighted in this research study.

Though the active sites of LeuRS are highly conserved, the results indicated that there was sequential and structural variation that occurred mainly within the scaffold residues. Particularly scaffold residues within the CP1 and C-terminal domains. A dramatic example of LeuRS structural variation were the superimposed structures of E. coli LeuRS and S. cerevisiae LeuRS enzymes, which was not surprising given that these enzymes originate from organisms in different domains of life and are separated by approximately 1.5 billion years of evolutionary time. ${ }^{14,40}$ Accordingly, these enzymes had the most sequence and structural variation compared with the other enzymes in this research study, which is likely due to non-translational functionality assumed by LeuRS enzymes through eukaryotic speciation to facilitate complex biological processes not present in bacteria. However, with this noted, the active sites for these enzymes were highly conserved, supporting the hypothesis that LeuRS enzymes diverged from a common ancestor aaRS., 9

Additional sequential and structural comparisons of LeuRS enzymes were generated, determining that through the speciation of eukaryotes there were only minor evolutionary changes. This was especially apparent when comparing LeuRS enzymes amongst higher-order eukaryotes. Comparisons between A. carolinensis LeuRS : T. guttate LeuRS and M. musculus LeuRS : H. sapiens LeuRS indicated very minor structural deviations. This result could be due to the relative short amount of evolutionary time between these organisms as well as their close physiologies. ${ }^{51-53}$ Contrasting this result with the lower-order organisms, the LeuRS enzymes within this group displayed more variability. This is likely due to longer evolutionary time spans between these organisms as well as dissimilar physiologies. ${ }^{40}$ Thus, the eukaryotic lineage carefully maintained LeuRS structure through the speciation process with the actives sites being strictly conserved.

\section{CONCLUSIONS}

These research findings show a high level of sequence and structural conservation amongst the eukaryotic LeuRS enzymes that were examined. Evolutionarily, this would indicate that vertical gene transfer events fostered the propagation of LeuRS through the speciation of eukaryotes. Unlike what has been reported for the bacterial and Archaeal domains, which have both been shown to have experienced LeuRS HGT and domain acquisition events. ${ }^{7-9}$ Additionally, both bacterial and Archaeal domains have robust mechanisms in place to facilitate HGT events. Thus, these results are expected, especially since the Eukaryota domain was the last domain to have emerged through the evolution of life and is more molecularly complex. ${ }^{40}$ Therefore, gene transfer events in eukaryotic cells are more restrictive than gene transfer events in bacteria or Archaea, thus the likelihood of LeuRS HGT events occurring amongst eukaryotes is almost non-existent. Especially due to LeuRS being part of the translational machinery. Enzymes that mechanistically drive the molecular central dogma were likely acquired before or at the time of LUCA, thus many of them would phylogenetically be deeply rooted at the base of the tree of life. Therefore, later acquisition of these enzymes by eukaryotes is improbable. As such, we propose that LeuRS was transferred through the eukaryotic speciation process via vertical gene transfer, which explains the high rate of LeuRS conservation within each taxa of the eukaryotic domain.

\section{ACKNOWLEDGEMENTS}

The authors thank the University of Nebraska system for supporting this research effort. 


\section{REFERENCES}

1. O'Donoghue, P.; Luthey-Schulten, Z., (2003) On the evolution of structure in aminoacyl-tRNA synthetases. Microbiol Mol Biol Rev, 67 (4), 550-573.

2. Lee, N.; Bessho, Y.; Wei, K.; Szostak, J. W.; Suga, H., (2000) Ribozyme-catalyzed tRNA aminoacylation. Nat Struct Biol, 7 (1), 28-33.

3. Arnez, J. G.; Moras, D., (1997) Structural and functional considerations of the aminoacylation reaction. Trends Biochem Sci, $22(6), 211-216$.

4. Ofengand, E. J.; Dieckmann, M.; Berg, P., (1961) The enzymic synthesis of amino acyl derivatives of ribonucleic acid. III. Isolation of amino acid-acceptor ribonucleic acids from Escherichia coli. J Biol Chem, 236, 1741-1747.

5. Woese, C. R.; Olsen, G. J.; Ibba, M.; Soll, D., (2000) Aminoacyl-tRNA synthetases, the genetic code, and the evolutionary process. Microbiol Mol Biol Rev, 64 (1), 202-236.

6. Wolf, Y. I.; Aravind, L.; Grishin, N. V.; Koonin, E. V., (1999) Evolution of aminoacyl-tRNA synthetases--analysis of unique domain architectures and phylogenetic trees reveals a complex history of horizontal gene transfer events. Genome Res, 9 (8), 689-710.

7. Fournier, G. P.; Andam, C. P.; Alm, E. J.; Gogarten, J. P., (2011) Molecular evolution of aminoacyl tRNA synthetase proteins in the early history of life. Orig Life Evol Biosph, 41 (6), 621-632.

8. Andam, C. P.; Harlow, T. J.; Papke, R. T.; Gogarten, J. P., (2012) Ancient origin of the divergent forms of leucyl-tRNA synthetases in the Halobacteriales. BMC Evol Biol, 12, 85.

9. Dohm, J. C.; Vingron, M.; Staub, E., (2006) Horizontal gene transfer in aminoacyl-tRNA synthetases including leucine-specific subtypes. J Mol Evol, 63 (4), 437-447.

10. Eriani, G.; Delarue, M.; Poch, O.; Gangloff, J.; Moras, D., (1990) Partition of tRNA synthetases into two classes based on mutually exclusive sets of sequence motifs. Nature, 347 (6289), 203-206.

11. Ibba, M.; Curnow, A. W.; Soll, D., (1997) Aminoacyl-tRNA synthesis: divergent routes to a common goal. Trends Biochem Sci, $22(2), 39-42$.

12. Burbaum, J. J.; Schimmel, P., (1991) Assembly of a class I tRNA synthetase from products of an artificially split gene. Biochemistry, 30 (2), 319-324.

13. Cusack, S., (1997) Aminoacyl-tRNA synthetases. Curr Opin Struct Biol, 7 (6), 881-889.

14. Woese, C. R.; Kandler, O.; Wheelis, M. L., (1990) Towards a natural system of organisms: proposal for the domains Archaea, Bacteria, and Eucarya. Proc Natl Acad Sci U S A, 87 (12), 4576-4579.

15. Brown, J. R.; Doolittle, W. F., (1995) Root of the universal tree of life based on ancient aminoacyl-tRNA synthetase gene duplications. Proc Natl Acad Sci U S A, 92 (7), 2441-2445.

16. Schimmel, P.; Ribas de Pouplana, L., (1995) Transfer RNA: from minihelix to genetic code. Cell, 81 (7), $983-986$.

17. Schmitt, E.; Meinnel, T.; Blanquet, S.; Mechulam, Y., (1994) Methionyl-tRNA synthetase needs an intact and mobile 332KMSKS336 motif in catalysis of methionyl adenylate formation. J Mol Biol, 242 (4), 566-576.

18. Webster, T.; Tsai, H.; Kula, M.; Mackie, G. A.; Schimmel, P., (1984) Specific sequence homology and three-dimensional structure of an aminoacyl transfer RNA synthetase. Science, 226 (4680), 1315-1317.

19. Cusack, S.; Yaremchuk, A.; Tukalo, M., (2000) The 2 A crystal structure of leucyl-tRNA synthetase and its complex with a leucyl-adenylate analogue. EMBO J, 19 (10), 2351-2361.

20. Eriani, G.; Cavarelli, J.; Martin, F.; Ador, L.; Rees, B.; Thierry, J. C.; Gangloff, J.; Moras, D., (1995) The class II aminoacyltRNA synthetases and their active site: evolutionary conservation of an ATP binding site. J Mol Evol, 40 (5), $499-508$.

21. Zhao, M. W.; Zhu, B.; Hao, R.; Xu, M. G.; Eriani, G.; Wang, E. D., (2005) Leucyl-tRNA synthetase from the ancestral bacterium Aquifex aeolicus contains relics of synthetase evolution. EMBO J, 24 (7), 1430-1439.

22. Iwabe, N.; Kuma, K.; Hasegawa, M.; Osawa, S.; Miyata, T., (1989) Evolutionary relationship of archaebacteria, eubacteria, and eukaryotes inferred from phylogenetic trees of duplicated genes. Proc Natl Acad Sci U S A, 86 (23), 9355-9359.

23. Altschul, S. F.; Gish, W.; Miller, W.; Myers, E. W.; Lipman, D. J., (1990) Basic local alignment search tool. J Mol Biol, 215 (3), 403-410.

24. Sievers, F.; Wilm, A.; Dineen, D.; Gibson, T. J.; Karplus, K.; Li, W.; Lopez, R.; McWilliam, H.; Remmert, M.; Soding, J.; Thompson, J. D.; Higgins, D. G., (2011) Fast, scalable generation of high-quality protein multiple sequence alignments using Clustal Omega. Mol Syst Biol, 7, 539.

25. Simossis, V. A.; Heringa, J., (2005) PRALINE: a multiple sequence alignment toolbox that integrates homology-extended and secondary structure information. Nucleic Acids Res, 33 (Web Server issue), W289-294.

26. Chan, P. P.; Lowe, T. M., (2009) GtRNAdb: a database of transfer RNA genes detected in genomic sequence. Nucleic Acids Res, 37 (Database issue), D93-97.

27. Di Tommaso, P.; Moretti, S.; Xenarios, I.; Orobitg, M.; Montanyola, A.; Chang, J. M.; Taly, J. F.; Notredame, C., (2011) TCoffee: a web server for the multiple sequence alignment of protein and RNA sequences using structural information and homology extension. Nucleic Acids Res, 39 (Web Server issue), W13-17.

28. Yang, Y.; Faraggi, E.; Zhao, H.; Zhou, Y., (2011) Improving protein fold recognition and template-based modeling by 
employing probabilistic-based matching between predicted one-dimensional structural properties of query and corresponding native properties of templates. Bioinformatics, 27 (15), 2076-2082.

29. Yang, J.; Zhang, Y., (2015) I-TASSER server: new development for protein structure and function predictions. Nucleic Acids Res, 43 (W1), W174-181.

30. Yang, J.; Yan, R.; Roy, A.; Xu, D.; Poisson, J.; Zhang, Y., (2015) The I-TASSER Suite: protein structure and function prediction. Nat Methods, 12 (1), 7-8.

31. Pettersen, E. F.; Goddard, T. D.; Huang, C. C.; Couch, G. S.; Greenblatt, D. M.; Meng, E. C.; Ferrin, T. E., (2004) UCSF Chimera--a visualization system for exploratory research and analysis. J Comput Chem, 25 (13), 1605-1612.

32. Sanner, M. F.; Olson, A. J.; Spehner, J. C., (1996) Reduced surface: an efficient way to compute molecular surfaces. Biopolymers, 38 (3), 305-320.

33. Harris, M.; Jones, T. A., (2001) Molray--a web interface between O and the POV-Ray ray tracer. Acta Crystallogr D Biol Crystallogr, 57 (Pt 8), 1201-1203.

34. Letunic, I.; Bork, P., (2007) Interactive Tree Of Life (iTOL): an online tool for phylogenetic tree display and annotation. Bioinformatics, 23 (1), 127-128.

35. Letunic, I.; Bork, P., (2016) Interactive tree of life (iTOL) v3: an online tool for the display and annotation of phylogenetic and other trees. Nucleic Acids Res, 44 (W1), W242-245.

36. Schimmel, P., (2008) Development of tRNA synthetases and connection to genetic code and disease. Protein Sci, 17 (10), 1643-1652.

37. Palencia, A.; Crepin, T.; Vu, M. T.; Lincecum, T. L., Jr.; Martinis, S. A.; Cusack, S., (2012) Structural dynamics of the aminoacylation and proofreading functional cycle of bacterial leucyl-tRNA synthetase. Nat Struct Mol Biol, 19 (7), $677-684$.

38. Schimmel, P. R.; Soll, D., (1979) Aminoacyl-tRNA synthetases: general features and recognition of transfer RNAs. Annu Rev Biochem, 48, 601-648.

39. Mursinna, R. S.; Lincecum, T. L., Jr.; Martinis, S. A., (2001) A conserved threonine within Escherichia coli leucyl-tRNA synthetase prevents hydrolytic editing of leucyl-tRNALeu. Biochemistry, 40 (18), 5376-5381.

40. Moreira, D.; Lopez-Garcia, P., (2015) Evolution of viruses and cells: do we need a fourth domain of life to explain the origin of eukaryotes? Philos Trans R Soc Lond B Biol Sci, 370 (1678), 20140327.

41. Gogarten, J. P.; Kibak, H.; Dittrich, P.; Taiz, L.; Bowman, E. J.; Bowman, B. J.; Manolson, M. F.; Poole, R. J.; Date, T.; Oshima, T.; et al., (1989) Evolution of the vacuolar H+-ATPase: implications for the origin of eukaryotes. Proc Natl Acad Sci U S A, 86 (17), 6661-6665.

42. Schimmel, P.; Ribas de Pouplana, L., (2001) Formation of two classes of tRNA synthetases in relation to editing functions and genetic code. Cold Spring Harb Symp Quant Biol, 66, 161-166.

43. Kumar, M.; Kumar, S. A.; Dimkovikj, A.; Baykal, L. N.; Banton, M. J.; Outlaw, M. M.; Polivka, K. E.; Hellmann-Whitaker, R. A., (2015) Zinc is the molecular "switch" that controls the catalytic cycle of bacterial leucyl-tRNA synthetase. J Inorg Biochem, 142, 59-67.

44. Fersht, A. R., (1998) Sieves in sequence. Science, 280 (5363), 541.

45. Hellmann, R. A.; Martinis, S. A., (2009) Defects in transient tRNA translocation bypass tRNA synthetase quality control mechanisms. J Biol Chem, 284 (17), 11478-11484.

46. Pang, Y. L.; Martinis, S. A., (2009) A paradigm shift for the amino acid editing mechanism of human cytoplasmic leucyl-tRNA synthetase. Biochemistry, 48 (38), 8958-8964.

47. Guo, M.; Schimmel, P., (2013) Essential nontranslational functions of tRNA synthetases. Nat Chem Biol, 9 (3), $145-153$.

48. Bonfils, G.; Jaquenoud, M.; Bontron, S.; Ostrowicz, C.; Ungermann, C.; De Virgilio, C., (2012) Leucyl-tRNA synthetase controls TORC1 via the EGO complex. Mol Cell, 46 (1), 105-110.

49. Han, J. M.; Jeong, S. J.; Park, M. C.; Kim, G.; Kwon, N. H.; Kim, H. K.; Ha, S. H.; Ryu, S. H.; Kim, S., (2012) Leucyl-tRNA synthetase is an intracellular leucine sensor for the mTORC1-signaling pathway. Cell, 149 (2), 410-424.

50. Duran, R. V.; Hall, M. N., (2012) Leucyl-tRNA synthetase: double duty in amino acid sensing. Cell Res, 22 (8), $1207-1209$.

51. Padian, K.; Chiappe, L. M., (1998) The origin of birds and their flight. Sci Am, 278 (2), 38-47.

52. Nei, M.; Xu, P.; Glazko, G., (2001) Estimation of divergence times from multiprotein sequences for a few mammalian species and several distantly related organisms. Proc Natl Acad Sci U S A, 98 (5), 2497-2502.

53. Green, R. E.; Braun, E. L.; Armstrong, J.; Earl, D.; Nguyen, N.; Hickey, G.; Vandewege, M. W.; St John, J. A.; CapellaGutierrez, S.; Castoe, T. A.; Kern, C.; Fujita, M. K.; Opazo, J. C.; Jurka, J.; Kojima, K. K.; Caballero, J.; Hubley, R. M.; Smit, A. F.; Platt, R. N.; Lavoie, C. A.; Ramakodi, M. P.; Finger, J. W., Jr.; Suh, A.; Isberg, S. R.; Miles, L.; Chong, A. Y.; Jaratlerdsiri, W.; Gongora, J.; Moran, C.; Iriarte, A.; McCormack, J.; Burgess, S. C.; Edwards, S. V.; Lyons, E.; Williams, C.; Breen, M.; Howard, J. T.; Gresham, C. R.; Peterson, D. G.; Schmitz, J.; Pollock, D. D.; Haussler, D.; Triplett, E. W.; Zhang, G.; Irie, N.; Jarvis, E. D.; Brochu, C. A.; Schmidt, C. J.; McCarthy, F. M.; Faircloth, B. C.; Hoffmann, F. G.; Glenn, T. C.; Gabaldon, T.; Paten, B.; Ray, D. A., (2014) Three crocodilian genomes reveal ancestral patterns of evolution among archosaurs. Science, 346 (6215), 1254449. 


\section{ABOUT THE STUDENT AUTHORS}

Katelin Arndt is currently attending the University of Nebraska, Kearney with a Comprehensive Biology major with an emphasis in Health Science. She is a part of the honors program and a member of Phi Eta Sigma. She is planning a career as a physician assistant. Katelin started undergraduate research under Doctor Rachel Whitaker in the fall of 2016 and hopes to continue research both throughout her academic career and as a medical professional.

Katelyn Unvert is a sophomore majoring in biology at the University of Nebraska, Kearney. She has previously published her research in the Journal of Inorganic Biochemistry and has presented this research at a national conference. She plans to continue to participate in the scientific process by conducting undergraduate research. Her future plans are to attend pharmacy school and conduct pharmacological research.

\section{PRESS SUMMARY}

Enzymes known as Aminoacly-tRNA synthetases are an important part of every known cell. Because they are so widespread, there is considerable research that can be done on how this enzyme has evolved over time. This study focuses on the specific enzyme leucyl-tRNA synthetase. It shows how the enzyme has changed, what aspects of it are essential to its function, and that it may have potential in pharmaceuticals. 


\title{
The Effect of $\mathrm{CO}_{2}$, Intracellular $\mathrm{pH}$ and Extracellular $\mathrm{pH}$ on Mechanosensory Proprioceptor Responses in Crayfish and Crab
}

\author{
Viresh Dayaram, Cole Malloy, Sarah Martha, Brenda Alvarez, Ikenna Chukwudolue, Nadera Dabbain, Dlovan Mahmood, Slavina Goleva, Tori \\ Hickey, Angel Ho, Molly King, Paige Kington, Matthew Mattingly, Samuel Potter, Landon Simpson, Amanda Spence, Henry Uradu, Jacob Van \\ Doorn, dlovan faiq, Robin L. Cooper \\ Department of Biology, University of Kentucky, Lexington, KY \\ Students:vda223@g.uky.edu*,malloycole@gmail.com,sarah.martha@gmail.com,brenda.alvarez@uky.edu,ikenna.chuk.wudolue@uky.edu, \\ Nida222@g.uky.edu,dlovan.kurdi@uky.edu,slavinag@gmail.com,tori.bickey@uky.edu,angel.ho@uky.edu,molly.king@uky.edu, \\ paige.kington@uky.edu,matthew.mattingly3@uky.edu,samuel.potter@uky.edu,4landonsimpson@uky.edu, amanda.spence@uky.edu, \\ benry.uradu@uky.edu,jay.vandoorn@uky.edu,Dlovanf@gmail.com
}

Mentor:RLCOOP1@uky.edut

\begin{abstract}
Proprioceptive neurons monitor the movements of limbs and joints to transduce the movements into electrical signals. These neurons function similarly in species from arthropods to humans. These neurons can be compromised in disease states and in adverse environmental conditions such as with changes in external and internal $\mathrm{pH}$. We used two model preparations (the crayfish muscle receptor organ and a chordotonal organ in the limb of a crab) to characterize the responses of these proprioceptors to external and internal $\mathrm{pH}$ changes as well as raised $\mathrm{CO}_{2}$. The results demonstrate the proprioceptive organs are not highly sensitive to changes in extracellular $\mathrm{pH}$, when reduced to 5.0 from 7.4. However, if intracellular $\mathrm{pH}$ is decreased by exposure to propionic acid or saline containing $\mathrm{CO}_{2}$, there is a rapid decrease in firing rate in response to joint movements. The responses recover quickly upon reintroduction of normal $\mathrm{pH}$ (7.4) or saline not tainted with $\mathrm{CO}_{2}$. These basic understandings may help to address the mechanistic properties of mechanosensitive receptors in other organisms, such as muscle spindles in skeletal muscles of mammals and tactile as well as pressure (i.e., blood pressure) sensory receptors.
\end{abstract}

\section{KEYWORDS}

Proprioception; Sensory; Invertebrate; Carbon Dioxide; Protons; Mechanosensory; Intracellular pH; Extracellular pH

\section{INTRODUCTION}

Cellular metabolism in animals produces $\mathrm{CO}_{2}$ as a by-product in the production of ATP, which is rapidly buffered or eliminated to maintain a homeostatic balance in dissolved $\mathrm{CO}_{2}, \mathrm{pH}$ and $\mathrm{HCO}_{3}$. The interrelationship between $\mathrm{pH}$ and $\mathrm{CO}_{2}$ goes beyond the equilibrium of $\mathrm{CO}_{2}+\mathrm{H}_{2} \mathrm{O} \leftrightarrow \mathrm{H}_{2} \mathrm{CO}_{3} \leftrightarrow \mathrm{HCO}_{3}{ }^{-}+\mathrm{H}^{+}, 3$ as $\mathrm{H}^{+}$ions are also in balance with other buffering mechanisms within cells as well as within the extracellular fluid (ECF). Cellular composition and ECF both contribute in many ways to buffering $\mathrm{H}^{+}$ as well as transporting $\mathrm{CO}_{2}$ in various states (soluble and $\mathrm{HCO}_{3}$ ). Each organelle and cytoplasm of cells maintains a different $\mathrm{pH}$ level. This demonstrates that $\mathrm{H}^{+}$balance is uniquely regulated by various process including pumps, exchangers, and transportors. ${ }^{4}$ In spite of experimentally lowering extracellular $\mathrm{pH}$, the cytoplasm of many cells will not match the extracellular $\mathrm{pH} .5,6 \mathrm{Thus,}$ it cannot be assumed that extracellular $\mathrm{pH}\left(\mathrm{pH}_{\mathrm{o}}\right)$ is in equilibrium with the $\mathrm{pH}$ in cells $\left(\mathrm{pH}_{\mathrm{i}}\right)$ as protons are not freely diffusible across the cell bilipid membrane.

Increased cellular metabolism for tissues (e.g. skeletal muscle or neural tissue) can have rapid effects on the blood/hemolymph $\mathrm{pH}$ and is usually dealt with quickly in reaching a homeostatic level. Some organisms can withstand wide ranges in $\mathrm{pH}^{\mathrm{H}}$ and $\mathrm{CO}_{2}$ levels within the blood/hemolymph without any apparent pathological conditions while other organisms cannot. An example of this is exhibited in honeybees, which can use a defense with group effort in producing $\mathrm{CO}_{2}$ and increasing temperature in order to kill an invading hornet as the honey bee can tolerate the acute changes in the environment. ${ }^{7}$ Early methods in mammalian anesthesia for surgery was to have patients rebreathe exhaled air resulting in increased $\mathrm{CO}_{2}$. This suggests an inability to withstand

\footnotetext{
${ }^{\dagger}$ This course project is part of a new trend in teaching science to undergraduates. ${ }^{1}$ Course-based undergraduate research experiences (CUREs) are relatively new and an approach being adopted by science educators in high schools and colleges. ${ }^{2}$ The course is neurophysiology lab (Bio446, Bio650).
} 
rapid changes in $\mathrm{CO}_{2}$ levels. ${ }^{8}$ It is not fully understood why some organisms are more resilient to $\mathrm{pH} / \mathrm{CO}_{2}$ alterations, but further elucidation in this field would benefit both general knowledge and could lead to potential therapeutics in mammalian pathological conditions (SIDS, COPD, ischemic conditions). Humans are known to display some acclimatization to altered $\mathrm{pH} / \mathrm{CO}_{2}$ balance with prolonged pathological conditions. It is generally acknowledged that people with chronic obstructive pulmonary disease (COPD) become less responsive to increased levels of blood $\mathrm{pCO}_{2}$ (low $\mathrm{pH}$ ) to drive breathing and become more driven by hypoxia $\left(\right.$ low $\mathrm{pO}_{2}$ ) for respiratory control.9-11 However, the effects on sensory neurons for proprioception in such altered conditions in humans has not been addressed as far as we are aware.

Abnormally low $\mathrm{pH}$ or higher $\mathrm{CO}_{2}$ in neural tissue can occur during pathological states (brain ischemia, hypercapnia, COPD) or by intentionally manipulating $\mathrm{CO}_{2}$ levels for research purposes. ${ }^{12-14} \mathrm{CO}_{2}$ is commonly used as an anesthetic in invertebrates. ${ }^{15,16}$ Many researchers utilizing invertebrates, particularly Drosopbila melanagaster, sort genetic strains under a $100 \% \mathrm{CO}_{2}$ anesthesia. The cellular response to high $\mathrm{CO}_{2}$ exposure or the resultant low $\mathrm{pH}$ may indeed have unintended consequences for physiological and behavioral studies. Exposing muscle or neurons to saline containing $100 \%$ bubbled $\mathrm{CO}_{2}$ rapidly decreases $\mathrm{pH}_{\mathrm{i}}$. With the use of ion sensitive electrodes, it has been found that the $\mathrm{pH}_{\mathrm{i}}$ in crab muscle and squid axons drops to around 6 and 5.7 respectively, when exposed to saline bubbled with $\mathrm{CO}_{2}{ }^{17-19}$ The $\mathrm{pH}_{\mathrm{o}}$ also decreases with $\mathrm{CO}_{2}$ bubbling, thus effects on cellular function may be due to extracellular or intracellular changes in $\mathrm{pH}$ or directly by $\mathrm{CO}_{2}$. Earlier studies indicate that molecular $\mathrm{CO}_{2}$, not the $\mathrm{pH}_{0}$ or $\mathrm{pH}_{\mathrm{i}}$ associated change, is in part responsible for alterations in synaptic receptivity at the neuromuscular junctions of crayfish and Drosophila malanagaster.18, 19

On the other hand, the action of protons $\left(\mathrm{H}^{+}\right)$themselves on cellular function is well established from effects on ion channels and pumps to enzymatic cascades. ${ }^{4}$ Protons can even increase the sensitivity of sensory neurons by increasing the opening of stretch activated ion channels (SACs), which are commonly used for mechanosensory transduction. ${ }^{20}$ The fundamental SACs are TRP channels (Transient Receptor Potential channels), DEG/ENaCs channels (Degenerin/epithelial sodium channels, known to be present in invertebrates and vertebrates), ${ }^{21}$ Piezo channels (pressure sensitive channels, found in plants and eukaryotic species) ${ }^{22}$ and TMC channels (transmembrane channels, sound- and vibration-sensing hair cells in mice). ${ }^{21}$ There are several reviews on SACs that provide more detailed descriptions of these channel types. ${ }^{21-23}$

Some SACs (i.e. DEG/ENaC channels) are altered by $\mathrm{pH}_{\circ} ;{ }^{20}$ however, we have shown that the SACs in the crustacean preparations used in this study did not have enhanced excitability in response to low $\mathrm{pH}_{\mathrm{o}}$ (5.0). To our knowledge no studies have yet addressed how $\mathrm{pH}_{\mathrm{i}}$ will affect $\mathrm{SACs}$ in joint proprioceptors of the crustaceans, insects, or mammals. We approached this challenge by using known methodological procedures to alter $\mathrm{pH}_{\mathrm{i}}$ by use of propionic acid as well as saline saturated with $\mathrm{CO}_{2}$ gas. 5, 24, 25 Propionic acid as well as $\mathrm{CO}_{2}$ will rapidly cross the bilipid membrane to acidify the cytoplasm; whereas altering the bathing saline $\mathrm{pH}$ to 5.0, with the use of $\mathrm{HCl}$, does not result in $\mathrm{pH}_{\mathrm{i}}$ becoming acidified in intact cells (see review). ${ }^{4}$ Our past study documented that acute exposure (1 to 2 minutes) to $\mathrm{pH}_{\circ}$ of 5.0 only showed a slight reduction in activity depending on the type displacements used; however, after one hour exposure overall sensitivity to the displacements was significantly reduced.

The two model preparations we chose to use for this study are the mechanoreceptors associated with sensory endings embedded within chordotonal organs (COs), which monitor joint movements in the limbs of arthropods (insects and crustaceans). Specifically, we used the crab propodite-dactylopodite joint (PD) organ and the muscle receptor organ (MRO) in the crayfish abdomen in our analysis. Since these two preparations are well-described model systems for mechanoreception ${ }^{26-32}$ we felt these preparations would be prudent to start to address the role of $\mathrm{pH}_{\mathrm{i}}, \mathrm{pH}_{\mathrm{o}}$ and the effect of $\mathrm{CO}_{2}$ on these joint proprioceptors.

The contribution of this study is to enhance the scientific understanding in the physiology of COs and the MRO in these crustacean preparations with regard to SACs and neuronal $\mathrm{pH}$ sensitivity by alterations in $\mathrm{pH}_{\mathrm{i}}, \mathrm{pH}_{\mathrm{o}}$ and $\mathrm{CO}_{2}$. The basic understandings may help to address the mechanistic underpinnings of mechanosensitive receptors in other organisms, such as muscle spindles in skeletal muscle of mammals and tactile as well as pressure (e.g., blood pressure) sensory receptors.

\section{METHODS AND PROCEDURES}

Crab

Blue crabs, Callinectes sapidus, were obtained from a local supermarket in Lexington, KY to which they were delivered from a distribution center in Atlanta, GA. They were bought and maintained in a sea water aquarium for several days prior to use in order to assess their health. All crabs used were alive and were very active upon autotomizing a leg for experimentation. While holding the crab with a net or large tongs across the carapace from behind, and avoiding the claws, a pinch across the merus of the walking leg with a pair of pliers would induce the leg to be autotomized. The leg was then placed in the Sylgard-lined dissecting dish and covered with crab saline at room temperature $\left(21^{\circ} \mathrm{C}\right)$. 
The chordotonal organ in the propodite-dactylopodite joint (PD) of the first or second walking legs of the crab was used. The details of the dissection and procedures are described in video and text. ${ }^{33}$ After exposing the PD nerve and pulling the nerve into a suction electrode for recording the nerve activity, the dactyl was moved throughout the extended and flexed positions for several cycles with the aid of a wooden probe to ensure the nerve was not pulling on the chordotonal strand. A length of the nerve was left out of the suction electrode to provide slack.

The experimental conditions consisted of moving the dactyl from a flexed $90^{\circ}$ angle from the propus to a full $0^{\circ}$ in an extended (or open) position and then released. When the dactyl was released the joint would obtain a partial flexed position. Prior to the next displacement, the joint was flexed to the same starting position. The rates of movements were $0.5 \mathrm{sec}, 1.0 \mathrm{sec}, 2.0 \mathrm{sec}$ and 4 sec for the $90^{\circ}$ displacement with $5 \mathrm{sec}$ between displacements. The analysis consisted of binning the responses into $0.5 \mathrm{sec}$ periods for all the displacements and obtaining a count of spikes or a firing frequency of the nerve.

\section{Crayfish}

Crayfish (Procambarus clarkil), measuring 6-10 cm in body length, were used throughout this study (Atchafalaya Biological Supply Co., Raceland, LA). They were housed individually in indoor tanks. The details of the dissection and procedures are described in video and text. ${ }^{34}$ The MRO nerve to either abdominal segment 2 or 3 was used in this study. The displacements used were from a relaxed position (similar to an extended abdomen in the intact animal) to a stretched position (similar to a flexed abdomen in the intact animal). The displacement rates were $0.5 \mathrm{sec}$ and $4 \mathrm{sec}$ for $5 \mathrm{~mm}$ distance. In addition, a stretch hold was used to obtain the static position sensitive response. The same electrode and signal recording technique was used as for the crab CO.

\section{Saline and pharmacology}

The salines used are the normal salines described previously. ${ }^{33,34}$ All bathing and experimental solutions were kept at the experimental room temperature of $21^{\circ} \mathrm{C}$. Propionic acid was diluted from a stock and mixed in the saline appropriate for the species used. $\mathrm{CO}_{2}$ was introduced by vigorously bubbling $100 \% \mathrm{CO}_{2}$ into the saline used for 10 minutes. The $\mathrm{pH}$ of the saline after bubbling was between 5.0 and 5.1. The saline was rapidly poured into the bathing dish and exchanged every 2 minutes with freshly $\mathrm{CO}_{2}$ bubbled saline. All chemical compounds were obtained from Sigma (St. Louis, $\mathrm{MO}$ ) and $\mathrm{CO}_{2}$ was purchased from a local supplier (Scott Gross, Lexington, KY).

\section{Electrophysiology}

Suction electrodes made from glass pipettes fitted with plastic tips were used to record extracelluar signals from the cut nerves. ${ }^{35}$ A P-15 amplifier (Grass Instruments) in conjunction with a PowerLab/4s A/D converter and Lab Chart 7 software (ADI Instruments, Colorado Springs, CO) obtained the signals to be recorded on a computer at a 10 or $20 \mathrm{kHz}$ sampling rate. All data are expressed as a mean $( \pm$ SEM$)$.

\section{To insure reproducibility in experimentation}

The data collected in the classroom with students using 8 different physiological rigs was preliminary data in order to obtain an idea of what to expect for the different experimental conditions. The students made the recordings and analyzed the data. For standardizing the rate of the movements and analysis, all the data presented in the manuscript was obtained by 2 people (one conducting the movements and one marking the files on the computer. Every experiment had 6 trials with different preparations and was conducted over the summer of 2016 in a month period. One individual (V.D.) analyzed all the data sets so analysis would be consistent. The movements of the joints were performed by the same individual (R.C) for all trails. The movements were made by physically moving the joint and counting out loud: "one Miss" ( $0.5 \mathrm{sec})$, "one Mississippi" (1 sec), "two Mississippi" (2 sec), etc. We timed verbal counting on a stopwatch several times to ensure consistency in the speed of counting. Each time a movement was started or stopped, a mark on the file with a tap on the key pad would be recorded. To be sure the static holds were correctly measured, a set time of 7 seconds was analyzed as indicated by a time stamp on the acquisition software.

\section{RESULTS}

The two model proprioceptive organs used in this study are characteristic for a variety of types of proprioceptive structures. The PD joint in the crab walking leg contains the chordotonal organ referred to as the PD chordotonal organ (Figure 1A). The structure consists of an elastic strand that is attached to the proximal end of the dactylopodite on one end. The other contact point spans the joint attaching to the closer apodeme (invertebrates' tendon like structure in which skeletal muscle attaches). The neuronal sensory endings are embedded within the elastic strand to detect the movement of the strand. The MRO is arranged differently in that the sensory endings are embedded within muscle fibers that span the joint of the abdomen (Figure 1B). Within the sensory endings of the PD organ and the MRO are the SACs, which initiate ionic flux and depolarization of the neuron when they are deformed by the mechanical forces placed on them. The neurons within the PD organ and MRO respond differently depending on the rate and direction of movement as well as the static position of the joint. Schematic diagrams of the movements used in this study are shown along with the representative neural activity recorded from the whole nerve (Figure 1). The PD joint 
was displaced from $90^{\circ}$ to $0^{\circ}$ at various rates $(0.5$ and 4.0 seconds). The same rates of movements were used for the MRO to provide a fast and a slow displacement. However, the anatomical arrangement is different so a direct correlation in firing rates of the neurons cannot be made between the two preparations. The general responses to the same environmental conditions can be compared. The displacement for the MRO was to a set position that mimicked flexion of the abdomen. Also, a static position of flexion (stretching of the MRO), which was held for 7 seconds, was used to index the neural activity and the effects of changing the extra or intracellular $\mathrm{pH}$ as well as the effects of exposure of $\mathrm{CO}_{2}$.
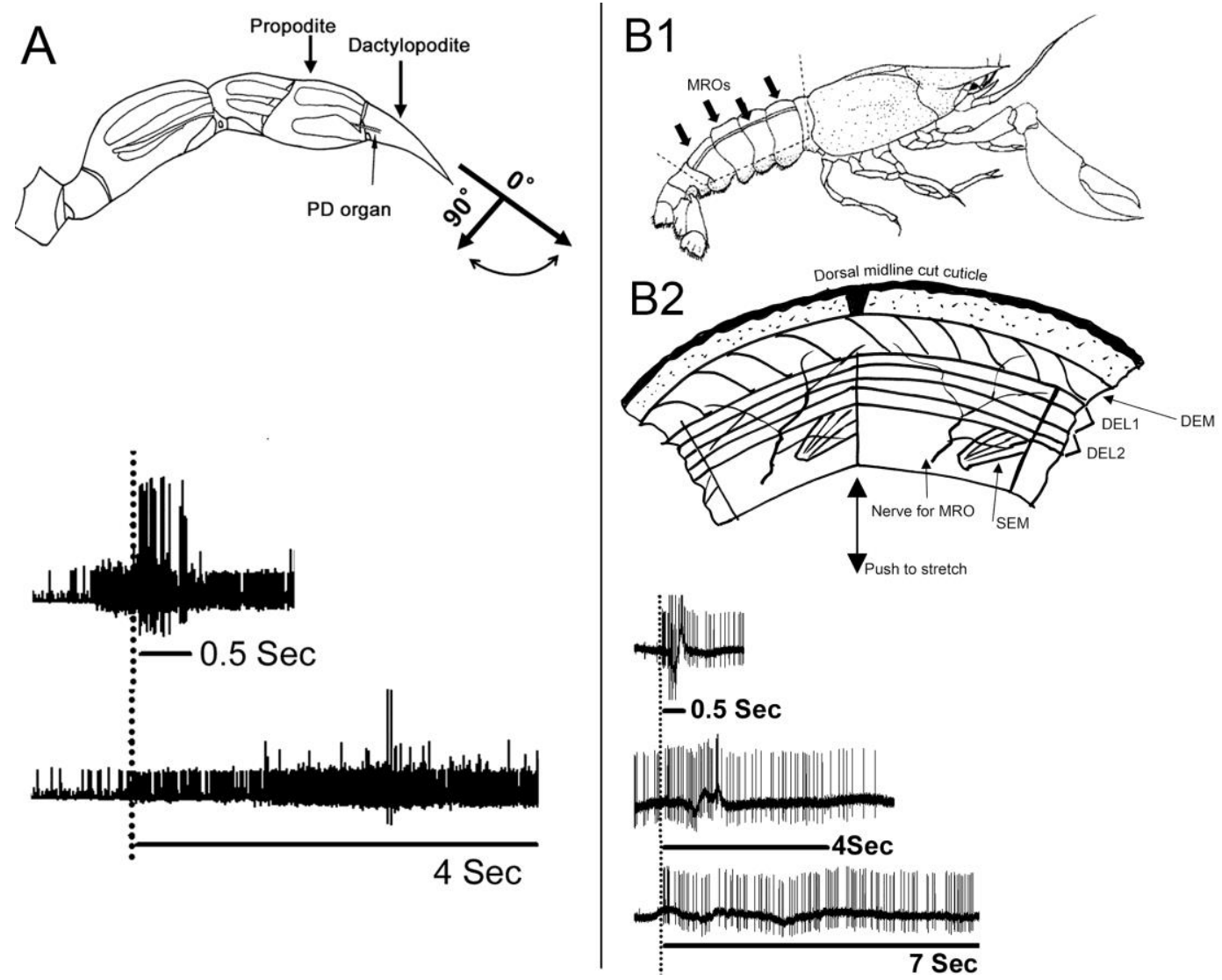

Figure 1. Anatomical arrangement of the displacements used for the PD organ of the crab walking leg (A) and the MRO of the crayfish abdomen (B). Either a stop pin or an anatomical position was used for consistency in the displacements. Rates of displacement for the crab joint were $0.5,1,2$, and 4 seconds from $90^{\circ}$ to fully extended $\left(0^{\circ}\right)$. B1: The MROs are located on the dorsal aspect of the abdomen. Movements for the MRO consisted of bending a joint in the hemilongitudinal segment of the abdomen to a set location at a rate of 0.5 or 4 seconds as well as stretched and held for 7 seconds. B2: Two abdominal segments are illustrated. A schematic view of the deep extensor muscles (looking from ventral to dorsal) is provided. The particular muscles identified: deep extensor medial

(DEM) muscles have a spiral fiber pattern, DEL1 is the first lateral group followed by the DEL2 muscles. The superficial extensor medial muscle (SEM) lies directly dorsal to DEL2. The two MRO muscles are more dorsal to the DEL1. The joint between the abdominal segments would be displaced at various rates to a set position while recording from the MRO nerve (the double arrow indicates where the joint between segments is located). Typical firing activity of the nerves is shown for a PD (top) and an MRO (bottom) preparation at each of the displacement rates. (Modified figure). ${ }^{65,66}$

Low $p H_{0}$

Acute exposure to the bathing media at $\mathrm{pH}$ of 5 decreased the PD organ sensitivity for the 2 and 4 second displacements but after an hour at $\mathrm{pH}_{\mathrm{o}}$ of 5 , the sensitivity to the 1,2 and 4 second displacements was also significantly reduced. The MRO did not decrease in responsiveness as much as the PD organ with the chronic exposure to low $\mathrm{pH}_{\mathrm{o}}$, but since there was such a wide variation in responses from preparation to preparation there was no consistent trend in the response to low $\mathrm{pH}_{\mathrm{o}}$. A representative plot showing the activity for saline, acute exposure to low $\mathrm{pH}_{\mathrm{o}}$, and chronic exposure $(1 \mathrm{hr})$ is shown in Figure $2 \mathrm{~A}$. The trend in this representative preparation indicates that $\mathrm{pH}_{\mathrm{o}}$ increased the spike activity; however, in comparing all 6 preparations there is substantial variability. Since each preparation was unique in the basal activity, a percent change within a preparation from saline exposure to low $\mathrm{pH}_{\mathrm{o}}$ exposure was calculated for the acute and chronic exposure to low $\mathrm{pH}_{\mathrm{o}}$ for all 6 preparations with the 3 displacements (Figure 2B). As indicated in Figure 2B, there was no consistent trend in increasing activity after acute exposure to low $\mathrm{pH}$. In comparing the crab PD with the crayfish MRO, the percent change in the number of spikes for the chronic exposure to low $\mathrm{pH}_{\mathrm{o}}$ is shown for the displacements, with the exception of showing the static hold for 7 seconds for the crayfish MRO since the static hold was not performed with the crab PD organ (Figure 2C). 

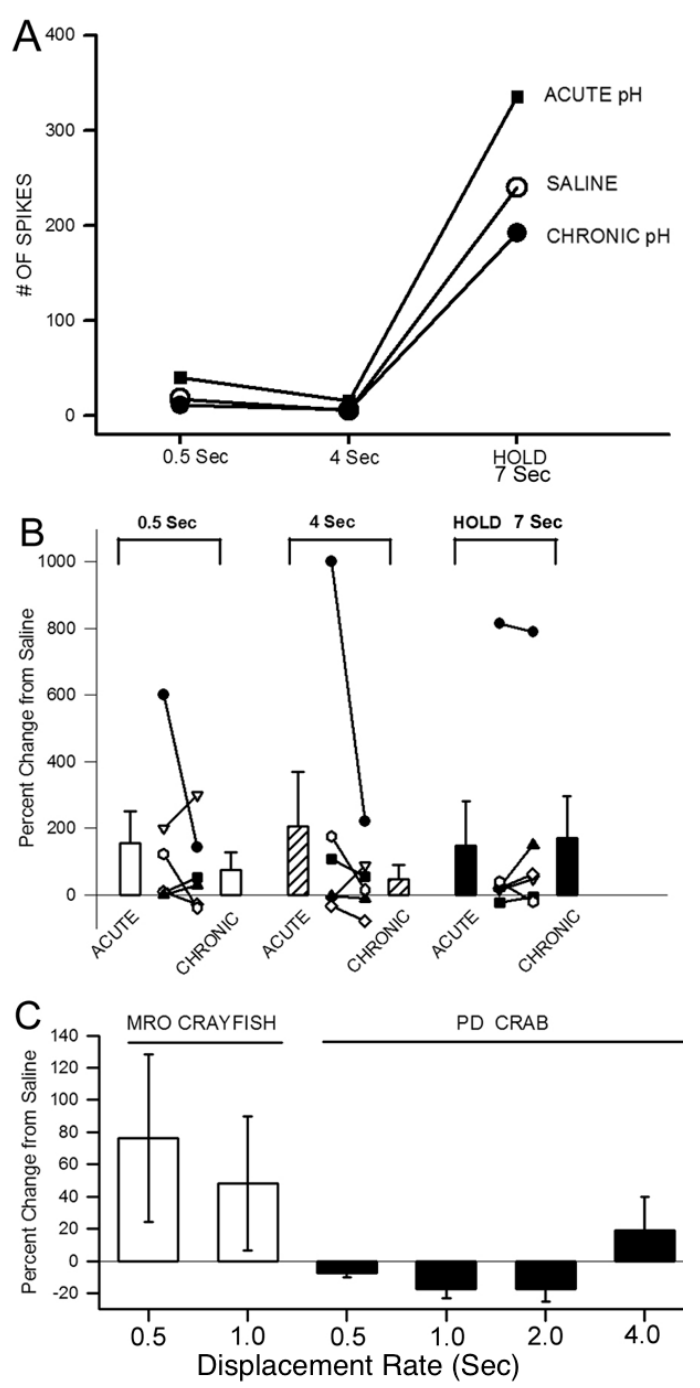

Figure 2. The effect of low $\mathrm{pH}_{\circ}$ on the sensitivity of joint proprioceptors. The rapid displacement within 0.5 second and 4 seconds did not demonstrate a large change in spiking differences for the MRO preparation (A). Static firing over a 7 second held position was also monitored for changes in spiking activity as shown for the MRO (A). A percent change from saline was used to compare the effects of low $\mathrm{pH}$ among preparations for the various displacement rates as shown for the MRO preparations (B). The same type of analysis was performed for the PD organ and the mean percent changes for both preparations are shown $(\mathbf{C})$.

\section{Propionic Acid}

A common technique used to rapidly reduce $\mathrm{pH}_{\mathrm{i}}$ is to expose preparations to saline containing propionic acid. ${ }^{4,5,24,25}$ This weak organic acid rapidly crosses the bilipid membrane and can also be exchanged back out of the cell by repeatedly changing the bathing media. A concentration of $20 \mathrm{mM}$ has been shown to produce a $\mathrm{pH}_{\mathrm{i}}$ of about 5.61.36,37 We freshly added propionic acid to the saline prior to bathing the preparations. The same displacement rates were used for exchanging the $\mathrm{pH}_{\mathrm{o}}$. Representative spike activity from a crayfish MRO preparation and a crab PD organ is shown (Figure 3 and 4, respectively). The neural activity generally ceased within 5 minutes for the crayfish MRO preparations of all 3 displacements $(0.5 \mathrm{sec}$ Figure 3A2, $4 \mathrm{sec}$ Figure 3B2, and the $7 \mathrm{sec}$ hold Figure 3C2). The effect was also rapid for the crab PD but there was still lingering activity after 5 minutes for the same displacement rates ( $0.5 \mathrm{sec}$ Figure 4A2, $4 \mathrm{sec}$ Figure 4B2, and the $7 \mathrm{sec}$ hold Figure 4C2). The range in neuronal responses, which displayed decreased activity throughout, implies that dynamic as well as static position sensitive neurons were affected. Both the crayfish and the crab preparations recovered well with exchanging the bathing saline a few times and repeating the displacement movements (see traces in A3, B3 and C3 in Figures 3 and 4). 


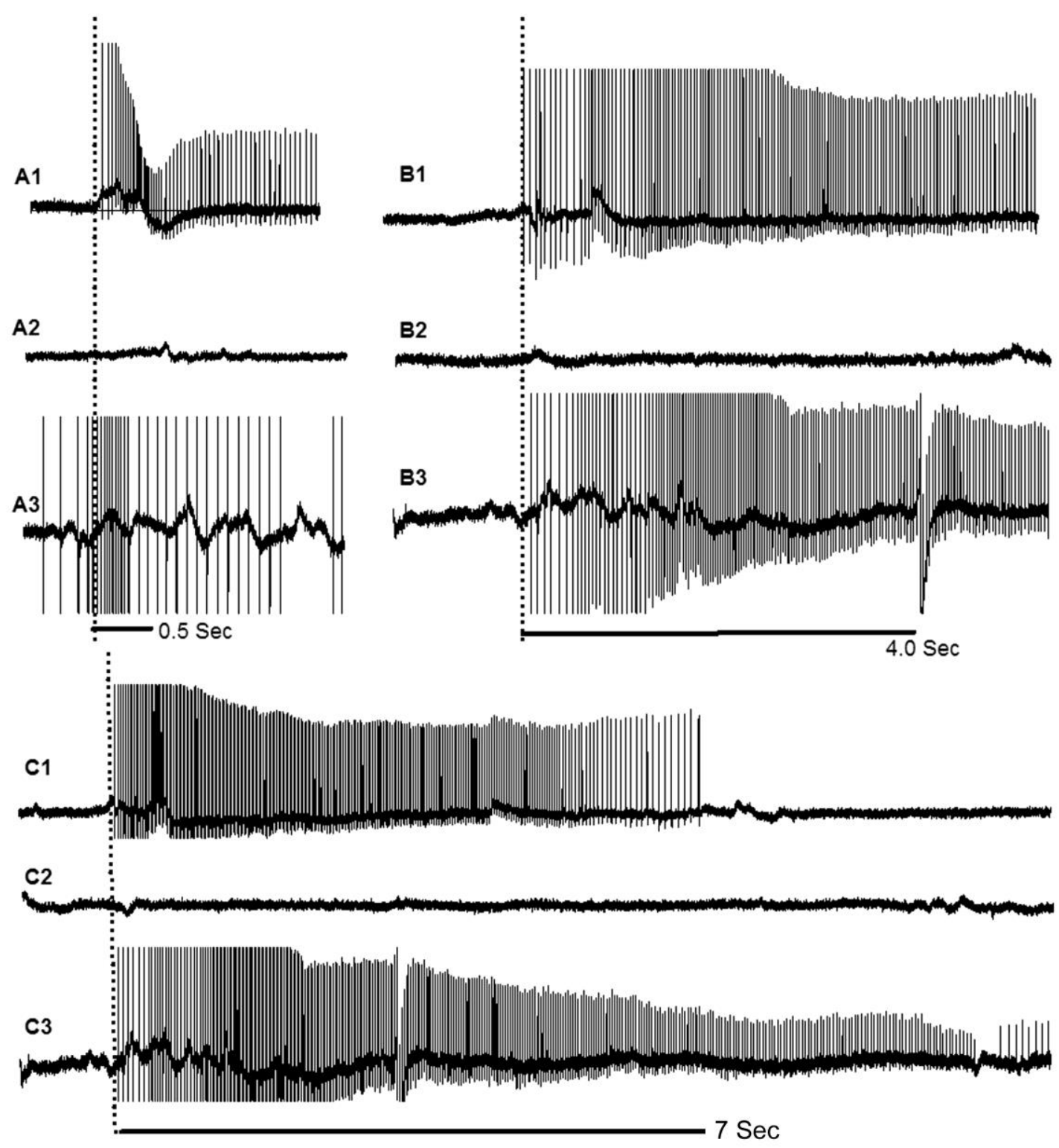

Figure 3. Representative traces in spiking for the different displacement rates and response to propionic acid exposure for the crayfish MRO. The 0.5 second displacement is shown in $\mathbf{A}$, while the 4 second is shown in $\mathbf{B}$, and the static held displacement of 7 seconds in shown in $\mathbf{C}$. The responses in normal saline (A1, B1, C1) and during exposure to $20 \mathrm{mM}$ propionic acid (A2, B2, C2) as well as wash out with a return to normal saline (A3, B3, C3) are shown. The decrease in amplitude in $\mathbf{C} 3$ is from the nerve being slightly displaced from recording electrode. 


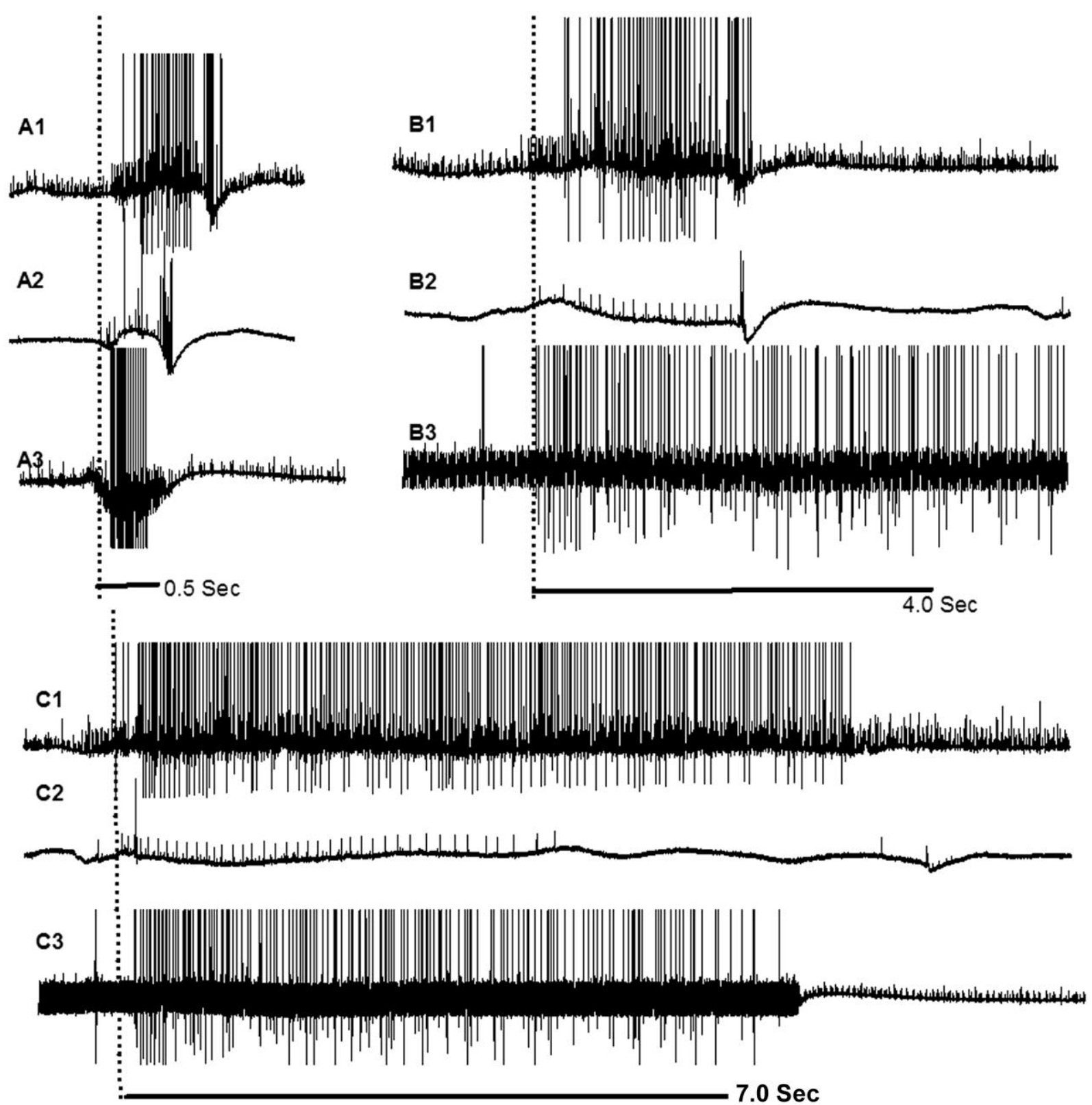

Figure 4. Representative traces in spiking for the different displacement rates and response to propionic acid exposure for the crab PD organ. The 0.5 second displacement is shown in $\mathbf{A}$, while the 4 second is shown in $\mathbf{B}$, and the static held displacement of 7 seconds in shown in $\mathbf{C}$. The responses in normal saline (A1,

B1, C1), during exposure to $20 \mathrm{mM}$ propionic acid (A2, B2, C2), and washed out with a return to normal saline (A3, B3, C3) are shown.

Since each preparation was unique in the basal activity, a percent change within a preparation to the saline exposure was calculated for the acute exposure to propionic acid for all 6 preparations with the 3 displacements for the crayfish MRO and crab PD organ. The trend in these preparations indicates that propionic acid reduces spike activity by nearly $100 \%$ in all 6 crayfish preparations and is greatly reduced in the crab PD for the 5-minute exposure (Figure 5).

Saline containing $\mathrm{CO}_{2}$

As with propionic acid, the dissolved $\mathrm{CO}_{2}$ in the saline rapidly crosses the bilipid membrane, perhaps quicker than propionic acid, where it acidifies the cell interior and reduces the $\mathrm{pH}$ in the saline bathing of the preparation. To maintain a high level of dissolved $\mathrm{CO}_{2}$, freshly bubbled saline was exchanged often with the bathing media. The same displacement rates and procedures were used to examine the effects of exposure to $\mathrm{CO}_{2}$ on the preparations. 


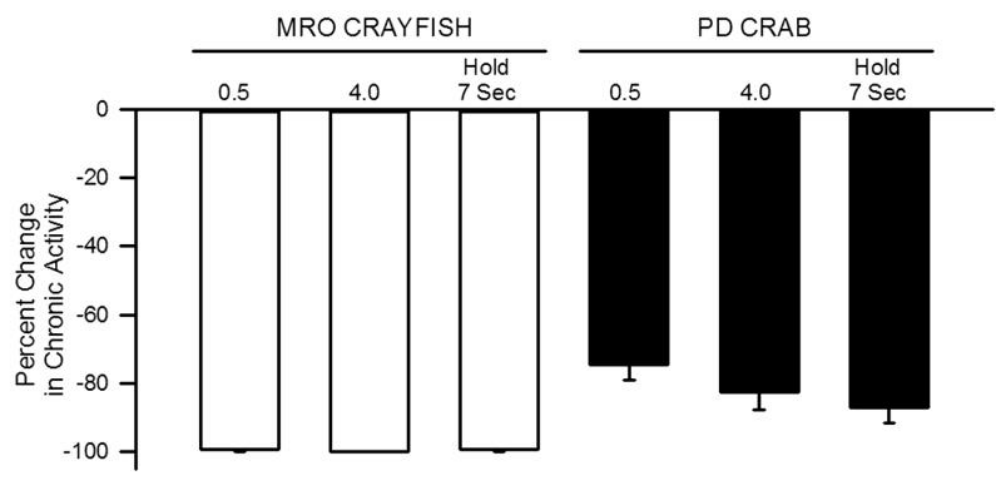

Figure 5. A percent change from saline was used to compare the MRO and PD preparations for the effects of propionic acid (20mM) for the various displacement rates and static held position.
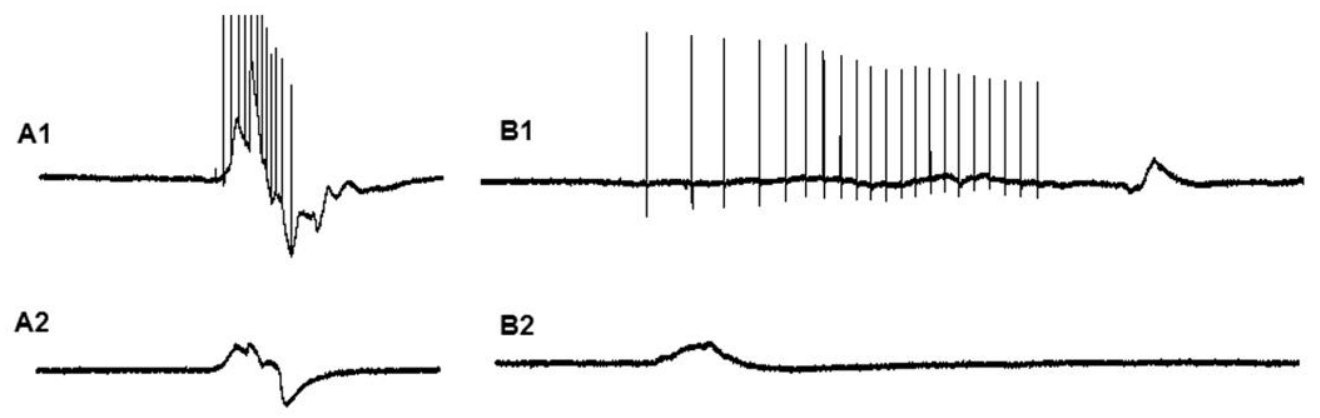

B2
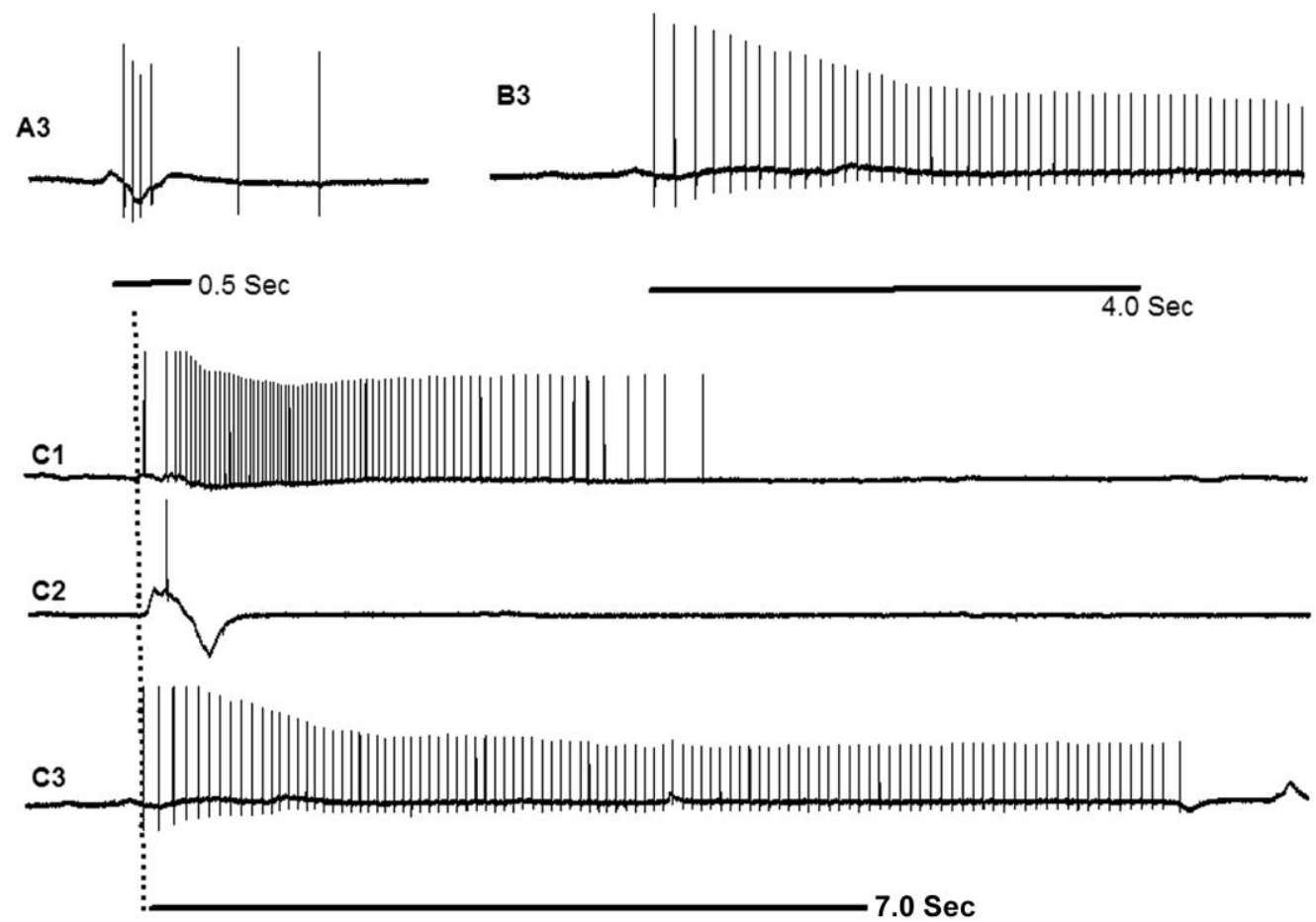

Figure 6. Representative traces in spiking for the different displacement rates and response to $\mathrm{CO}_{2}$-containing saline exposure for the crayfish MRO. The half second displacement is shown in $\mathbf{A}$, while the four second is shown in $\mathbf{B}$ and the static held displacement of 7 seconds in shown in $\mathbf{C}$. The responses in normal saline (A1, B1, C1) and during exposure to CO2 (A2, B2, C2) are shown along with wash out steps with a return to normal saline (A3, B3, C3). 
Representative MRO and a PD organ preparation are shown (Figure $6 \mathbf{\&} 7$ respectively). As with the exposure to propionic acid, the crayfish MRO preparations were more sensitive to $\mathrm{CO}_{2}$ exposure than the crab preparation, displaying a more robust decrease in activity. However, it must be noted that only 2 sensory neurons are monitored in the crayfish as compared to approximately 80 neurons in the crab preparation. Just a few spikes could still be measured in 3 crayfish preparations with rapid displacements whereas the other preparations completely ceased in activity. The crab preparations were substantially affected as well but some activity could still be measured in all the preparations during the $\mathrm{CO}_{2}$ exposure during one of the displacements rates $(0.5$ sec Figure 7A2, $4 \mathrm{sec}$ Figure 7B2, and the $7 \mathrm{sec}$ hold Figure 7C2) within the 5 minutes of exposure. Both the crayfish and the crab preparations recovered well upon saline exchange and repeated movements (see traces in A3, B3, and C3 in Figures $\mathbf{3}$ and $\mathbf{4}$ ). The recovery from $\mathrm{CO}_{2}$ was very rapid as compared to propionic acid exposure (i.e., recovery took place within approximately 1 minute after a single saline exchange vs. 3 or 4 exchanges of saline followed by a 5 to 10 minute waiting period after saline exchange from propionic acid in some cases).

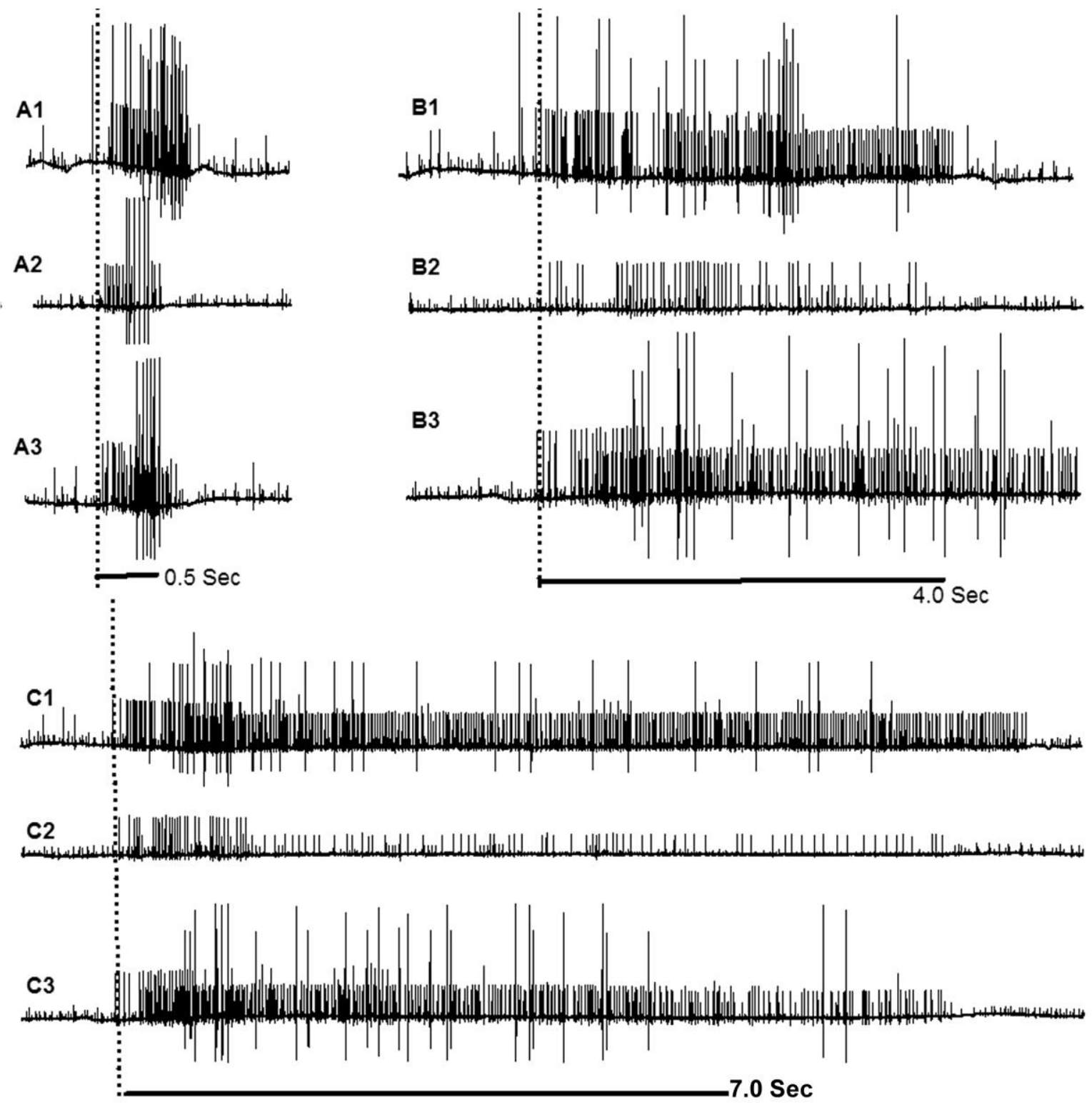

Figure 7. Representative traces in spiking for the different displacement rates and response to saline containing $\mathrm{CO}_{2}$ for the crab PD organ. The 0.5 second displacement is shown in $\mathbf{A}$, while the 4 second is shown in $\mathbf{B}$ and the static held displacement of 7 seconds in shown in $\mathbf{C}$. The responses in normal saline (A1, B1, C1), during exposure to CO2 (A2, B2, C2), and washed out with a return to normal saline (A3, B3, C3) are shown. 
As with exposure to low $\mathrm{pH}_{\mathrm{o}}$ and propionic acid, a percent change within a preparation to the saline exposure was calculated for the acute exposure for all 6 preparations with the 3 displacements for the crayfish MRO as well as the for the crab PD organ (Figure 8). The trend in these preparations indicates that $\mathrm{CO}_{2}$ decreased the spike activity in both sensory preparations $(6$ out 6 preparations). The overall percent reductions for the $\mathrm{CO}_{2}$ exposures are not as large as for the propionic acid exposure (compare

Figures 5 and 8 ).

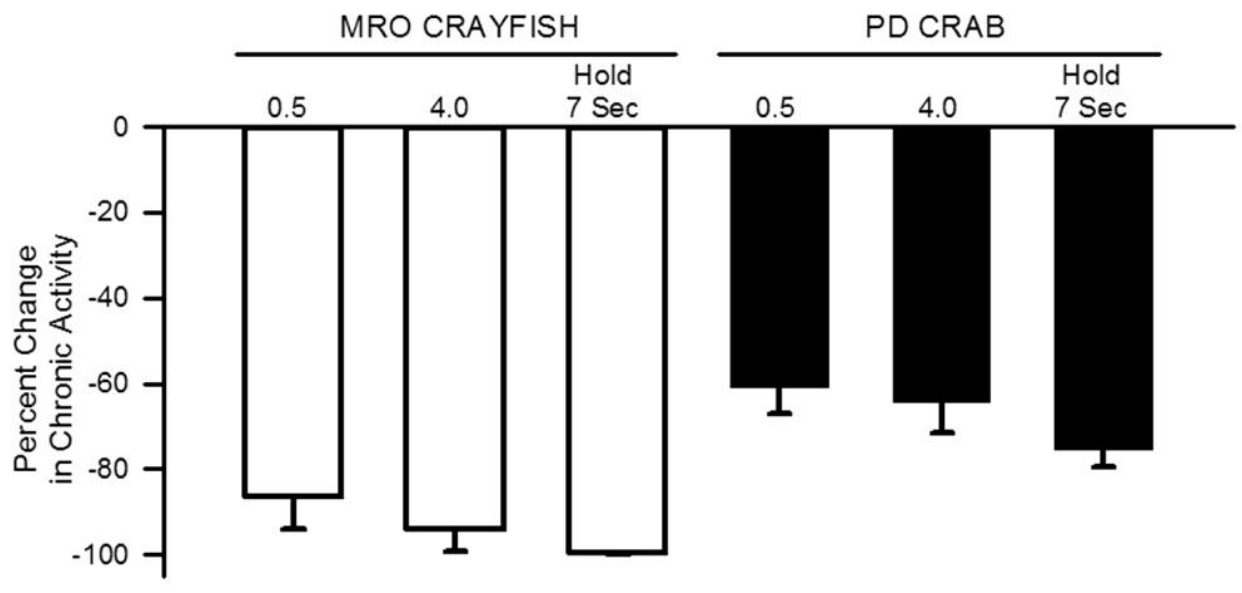

Figure 8. A percent change from saline was used to compare among the MRO and PD preparations for the effects of $\mathrm{CO}_{2}$ exposure for the various displacement rates and static held position.

\section{DISCUSSION}

In this study we demonstrated the proprioceptive neurons associated with the crayfish MRO preparation and the PD organ of the walking leg in the crab are not highly sensitive to changes in extracellular $\mathrm{pH}$, when $\mathrm{pH}_{\mathrm{o}}$ is reduced to 5.0 from 7.4. After this reduction, the preparations maintained their firing rate over various velocities of movement and static positions, even though a slight increase in activity was observed after acute exposure. However, if intracellular $\mathrm{pH}$ is decreased by exposure to propionic acid or saline containing $\mathrm{CO}_{2}$, there is a rapid decrease in firing rate in response to displacement. Recovery of activity is possible with re-exposure to normal physiological saline.

The exposure to high levels of $\mathrm{CO}_{2}$ occurs commonly on insects and crustaceans used in research laboratories since it is an anesthetic. It is often used to anesthetize flies to sort them in genetic analyses. $\mathrm{CO}_{2}$ induced $\mathrm{pH}$-related neuronal depression could play a role in developing newer anesthesia modalities for arthropods. Therefore, it is paramount to enhance understanding of the detriments that may arise because of chronic exposure to $\mathrm{CO}_{2}$. The chordotonal-proprioceptors within the joints of insects are analogous to the chordotonal-proprioceptors in the limbs of Crustacea. As stated earlier, the crayfish $\mathrm{MRO}$ is similar in structure and function to the muscle spindle proprioceptive organ in mammals, including humans. Since $\mathrm{CO}_{2}$ is a by-product of cellular metabolism throughout the animal kingdom, understanding how exposure to $\mathrm{CO}_{2}$ affects the two sensory model preparations used in this study can aid in addressing potential effects on neurons in other animals. Coupling energy metabolism and $\mathrm{H}^{+}$production within neural tissue was recently reviewed. ${ }^{38}$

The direct effects of $\mathrm{CO}_{2}$ are difficult to study due to the rapid buffering of $\mathrm{CO}_{2}$ and conversion to $\mathrm{HCO}_{3}+\mathrm{H}^{+} .^{3}$ In addition, the buffering abilities through proteins, organelle uptake and plasma membrane exchangers/pumps within neurons may vary..$^{4,38}$ The solubility of $\mathrm{CO}_{2}$ in solution varies with temperature and salinity so there are likely some differences in $\mathrm{CO}_{2}$ within the hemolymph when exposing freshwater crustaceans and salt-water crustaceans to environments of a given $\mathrm{CO}_{2}$ concentration. The higher osmolality saline used for the crab vs. the crayfish may also influence the amount of soluble $\mathrm{CO}_{2}$; however, the effects observed in this study on the sensory neurons are similar for both animal models. The rapid decrease in neuronal activity upon $\mathrm{CO}_{2}$ exposure, as with propionic acid, indicates a direct effect on either the SACs or in the ability of the neurons to produce action potentials or conduct electrical signals. Exposure to $\mathrm{CO}_{2}$ did not significantly decrease the amplitude of action potentials in motor neurons of the crayfish, as measured with intracellular electrodes. ${ }^{19}$ This suggests that the effects of $\mathrm{CO}_{2}$ on the proprioceptors may be primarily on mechanosensory transduction or on the initiation of an action potential. It was noted that crayfish axons did not have an alteration in voltage gated $\mathrm{Na}^{+}$channel activation with changes in $\mathrm{pH}_{\mathrm{o}} .{ }^{39} \mathrm{Thus}$, the induction of an action potential when past threshold should not be affected by $\mathrm{pH}_{\mathrm{o}}$ but the effect of $\mathrm{pH}_{\mathrm{i}}$ has not specifically been addressed in crab or crayfish neurons. However, it is known that depending on the state of activity of cells the effect of $\mathrm{CO}_{2}$ exposure may have different effects depending on the membrane potential. 40,41 
Since propionic acid and $\mathrm{CO}_{2}$ exposure produced similar results of decreasing the sensitivity of the proprioceptors to the displacements and that both compounds can reduce $\mathrm{pH}_{\mathrm{i}}$ rapidly, 4,5 we postulate that low $\mathrm{pH}_{\mathrm{i}}$ has an effect directly on the $\mathrm{SACs}$ or on the initiation of action potentials. Future investigations using direct injections of acid agents such as potassium acetate or $\mathrm{HCl}$, which are cell impermeant, could address this possibility. The hardiness of invertebrate preparations to conduct such experiments is well known. ${ }^{42-44}$

\section{Interactions between $\mathrm{pH}$ and other endogenous factors}

Decreasing $\mathrm{pH}_{\mathrm{o}}$ does not necessarily mean protons will diffuse into the cells and decrease $\mathrm{pH}_{\mathrm{i}}$ since the bilipid membrane is not freely permeable to protons although protons can travel through various channels when they are opened. Cells can maintain a basic state even with an exposure to an acidic bathing medium and upon injection or acidifying cells with various approaches (e.g., injection of $\mathrm{HCl}$, exposure to $\mathrm{CO}_{2}$ and $\mathrm{NH}_{4} \mathrm{Cl}$ pulses). They can readily return to a basic state following acidification of the surrounding environment. This indicates the strong nature of the cell to regulate proton balance through pumps and exchangers, ${ }^{6}$ as demonstrated for crayfish neurons. ${ }^{5}$ The low $\mathrm{pH}$ of 5 did not block the mechanical transduction of the stretch activated channels (SACs) in the sensory endings or the ability of the neurons to reach threshold and conduct action potentials. However, the fluidity of some bilipid membranes can be altered by small changes in $\mathrm{pH}_{\mathrm{o}}{ }^{45}$ The voltage gated $\mathrm{Na}^{+}$channel in the axon of crayfish appear to be insensitive to low $\mathrm{pH}_{\mathrm{o}}$ within the range which would block acid sensitive SACs. ${ }^{39}$ Additionally, we have shown in this study that these sensory endings and axons for the crayfish MRO and in the PD organ of the crab are not $\mathrm{pH}_{\mathrm{o}}$ sensitive. The ability to maintain sensory responsiveness and electrical activity of these neurons demonstrates the robust nature of these crustacean preparations to an acute acidic extracellular or hemolymph environment.

There are neuro-active substances within the hemolymph of crustaceans that can alter the activity of sensory neurons. An earlier study demonstrated that serotonin and ecdysone, on their own as well as in combination, altered the activity of the neurons associated with the crayfish MRO. ${ }^{46}$ The effects of serotonin and octopamine showed species differences in crustaceans in their effects on the MRO. ${ }^{47}$ Peetz \& Winter also reported on sustained activity in MROs when the hemolymph was mixed with a physiological saline, although no one particular substance was identified to be responsible for the action. ${ }^{48}$ As for the chordotonal organs in crabs, if the hemolymph is mixed with a physiological saline and used as the bathing solution, the preparations last longer when isolated from the animal. ${ }^{31}$ The species-specific saline without modulators or hemolymph present reduces unknown variables that may arise in an endogenous hemolymph. The effect of altered $\mathrm{pH}_{\mathrm{o}}$ or $\mathrm{pH}_{\mathrm{i}}$, by $\mathrm{CO}_{2}$ fluctuations, may vary within the animal due to buffering and the presence of neuromodulators within the hemolymph. In fact, we are not aware of physiological examination on the action of modulators over physiological measured ranges of $\mathrm{pH}$ known to occur within insects or crustaceans. In insects the $\mathrm{pH}$ of the hemolymph has been measured to be as low as 6.6 and for Drosophila the hemolymph (HL3) commonly used for physiology studies is $\mathrm{pH} 7.2$ as was measured from isolated pooled hemolymph samples. ${ }^{49}, 50$ However, it appears a physiological saline with a lower $\mathrm{pH}$ of 7.1 is substantially better for maintaining the heart function for exposed preparations. ${ }^{51}$ In addition, heart rates increased in response to serotonin with a bathing saline that was buffered and maintained at $7.1 .{ }^{51}$ In relation to mammals, we have not found any reports on the influence of $\mathrm{pH}_{\mathrm{o}}$ and alteration in the effects of neuromodulation by serotonin or dopamine on neuronal function in humans. Receptors for neurotransmitters such as acetylcholine, GABA, and glutamate receptors do show $\mathrm{pH}$ sensitivity. ${ }^{52}$ Several voltage gated ion channels also demonstrate $\mathrm{pH}$ sensitivity that is not assumed to be directly related to mechanical transduction. ${ }^{53}, 54$ Such effects on the neural excitability and electrical conduction of axons related to the crayfish MRO or proprioceptors in the crab have not been investigated. As we report, the SACs in the sensory endings do not appear to show a change in mechanosensory transduction with $\mathrm{pH}$ as low as 5.0 as measured by the frequency of the extracellular recorded spikes. The amplitude and shape of the action potentials may indeed be altered but we were not able to detect consistent differences by monitoring the spikes. However, the effect of $\mathrm{pH}_{\mathrm{o}}$ on neuronal activity depends on the type of neuron as some are known to be very sensitive to $\mathrm{pH}_{\mathrm{o}}$ changes which may occur due to indirect effects through influences on ion channels. ${ }^{55,56}$

Since lowering $\mathrm{pH}_{\mathrm{o}}$ does not directly address the effects of a lower $\mathrm{pH}_{\mathrm{i}}$, investigators have used various means to induce reductions in intracellular $\mathrm{pH}$ to address the physiological effects. Injecting substances: cell permeant compounds such as potassium acetate, propionic acid, or exposure to $\mathrm{CO}_{2}$, have all been utilized to reduce $\mathrm{pH}_{\mathrm{i}}{ }^{4}, 5,24,25,57$ Exposure to propionic acid as well as the bathing fluid containing $\mathrm{CO}_{2}$ rapidly decreases $\mathrm{pH}_{\mathrm{i}}$ from a basal state and the effect is rapidly reversed in removing these compounds. ${ }^{4,5}$ Since these compounds reduce $\mathrm{pH}_{\mathrm{i}}$ in addition to $\mathrm{pH}_{\mathrm{o}}$, any physiological alterations might be due to a combined effect, rather than a sole reduction in $\mathrm{pH}_{\mathrm{i}}$. The acute effect of propionic acid in our case was rapid and since the frequency of spikes drastically decreased we did not pursue the effects of repeated exposure times. Constant exposure for an hour, in propionic acid at the concentrations used, did not allow the neurons to recover. Long exposures to propionic acid may indeed cause effects that cannot be so readily reversed due to the ability of propionic acid to also transverse cellular organelles, such as mitochondria, within the cell. 
Comparative differences in the proprioceptors

Differences in the anatomical arrangement of the crayfish MRO and the crab PD can complicate direct comparisons with addressing effects on mechanosensory transduction since the sensory endings of the MRO are embedded within muscle. Thus, any effects on the excitatory or inhibitory motor neurons that innervate the muscles can alter the forces exerted on the sensory endings. However, the conditions addressed in this study with $\mathrm{pH}_{\mathrm{o}}, \mathrm{pH}_{\mathrm{i}}$ and $\mathrm{CO}_{2}$ showed similar outcomes for both preparations. More refined recordings in the graded responses within the sensory endings could be different for the MRO preparation exposed to low $\mathrm{pH}_{\mathrm{i}}$ or $\mathrm{pH}_{\mathrm{o}}$, by $\mathrm{HCl}$ adjusted saline or propionic acid, as compared to saline containing $\mathrm{CO}_{2}$. $\mathrm{CO}_{2}$ exposure was shown to block glutamate receptors at neuromuscular junctions whereas spontaneous events and evoked events are still present at neuromuscular junctions with low $\mathrm{pH}$ induced by other means. ${ }^{18,19}$ Thus, any decrease in baseline response in muscle tension from a lack of responding to the spontaneously released glutamate might reduce the basal tension on the sensory endings. Low $\mathrm{pH}_{\mathrm{o}}$ does produce some depolarization in muscle for insects and crustaceans, ${ }^{18,19}$ and thus could produce an increase in force production if the motor nerve was stimulated to produce muscle contraction. The conditions used in the current study were created by passively moving the joints and were not dependent on motor nerve stimulation, although any enhancement of the terminal depolarization, even in the transected motor axons, could have an impact on muscle contraction and tension on the sensory endings for the MRO preparation. These confounding factors do not arise in the crab PD preparation, as the sensory endings are readily exposed in the elastic strand connecting the propodite and dactylopodite. Additionally, the crayfish MRO within a hemi-segment is only comprised of two sensory neurons while the crab PD is comprised of around 80 different neurons. The sensory somata as well as the axons are much larger in the MRO preparations as compared to the PD neurons for the animals used in these studies. Buffering abilities within neurons of various sizes may be different and likely effects on input resistance of the cells are varied due to size differences. The depolarizations required to reach threshold for generating action potentials are likely different in the neurons of varied size.

\section{Potential translational implications}

In considering the possible translational implications of the findings in this study to those of mammals, one can readily relate them to neuronal pathophysiology in ischemia, hypoxia, and lowered $\mathrm{pH}_{\mathrm{o}}$ and $\mathrm{pH}_{\mathrm{i}}$ due to $\mathrm{CO}_{2}$ imbalances. ${ }^{58}$ An active area of research is elucidating the mechanisms behind a ketogenic diet decreasing the occurrences of epilepsy, ${ }^{59-61}$ which is known to be influenced by $\mathrm{pH}^{62}$

\section{CONCLUSIONS}

This analysis investigates potential mechanistic explanations for spreading depolarization and synaptic depression when tissue is not perfused well to relieve the altered $\mathrm{pH}$, ionic spillage, and $\mathrm{CO}_{2}$ accumulation which occurs from damaged cells or compartmentation due to swelling and reduced vascular perfusion outside the initial tramatome. ${ }^{63}$ Recent studies in addressing effects of neurons outside the site of initial injury focus on possible mechanisms, such as $\mathrm{K}^{+}$and other ion/intracellular component spillage, which would leak from damaged cells and could alter healthy cell excitability. ${ }^{64}$ However, little attention is given to the possible detriments of $\mathrm{CO}_{2}$ accumulation around surrounding cells. A synergistic effect may arise in situations in which injured cells dump intracellular ion and amino acid stores, leading to increased cellular activity through depolarization of healthy cell membranes, which could then enhance $\mathrm{CO}_{2}$ production and exacerbate cellular damage. As for muscle spindles associated with proprioception in mammals, a similar situation can arise. Compartmentalized muscle following injury lacks proper vascular perfusion. Thus, $\mathrm{CO}_{2}$ buildup may assist in damage, not only to the muscle fibers, but also to the sensory neurons positioned within the muscle spindles. Similar outcomes could theoretically arise in neural tissue following traumatic brain injury and/or in COPD patients who experience systemic alteration in $\mathrm{pH}$. It may be beneficial to conduct future studies which measure muscle spindle activity in muscles with damaged extrafusal fibers or in in situ preparations where solution $\mathrm{pH}$ can be altered experimentally.

\section{ACKNOWLEDGMENTS}

This work was funded by student laboratory fees for a course taught in Department of Biology, University of Kentucky and personal funds (RLC). 


\section{REFERENCES}

1. Linn, M.C., Palmer, E., Baranger, A., Gerard, E., Stone, E. (2015) Undergraduate research experiences: impacts and opportunities, Science 347, 1261757.

2. Bakshi, A., Patrick, L.E., Wischusen, E.W. (2016) A framework for implementing course-based undergraduate research experiences (CUREs) in freshman biology labs, The Amer Biol Teacher 78(6), 448-455. ISSN 0002-7685, electronic ISSN 19384211.

3. Stone, G.C., Koopowitz, H. (1974) Mechanisms of action of $\mathrm{CO}_{2}$ on the visual response of Galleria mellonella, J Insect Physiol 20, 485-496.

4. Putnam, R. (2001) Intracellular pH regulation. In: Sperelakis, N. (ed) Cell physiology sourcebook: a molecular approach, 3rd edn. Sect. II: Membrane potential transport physiology pumps, and exchangers. Academic Press, New York, pp 357-376.

5. Moody, W.J., Jr. (1981) The ionic mechanism of intracellular pH regulation in crayfish neurons, J Physiol 316, $293-308$.

6. Bevensee, M.O., Boron, W.F. (1998) pH regulation in mammalian neurons, in $p H$ and Brain Function (Kaila K. and Ransom B.R., Eds.) 211-231 Wiley-Liss, New York.

7. Sugahara, M., Sakamoto, F. (2009) Heat and carbon dioxide generated by honeybees jointly act to kill hornets, Naturwissenschaften 96(9), 1133-1136. doi: 10.1007/s00114-009-0575-0.

8. Eisele, J.H., Eger, E., Muallem, M. (1967) Narcotic properties of carbon dioxide in the dog, Anesthesiol 28, 856-865

9. Raurich, J.M., Rialp, G., Ibáñez, J., Ayestarán, I., Llompart-Pou, J.A., Togores, B. (2009). Hypercapnia test and weaning outcome from mechanical ventilation in COPD patients, Anaesth Intensive Care 37, 726-732.

10. Zapata, P., Larrain, C., Rivera, M., Calderon, C. (2009) Cardiovascular responses to hyperoxic withdrawal of arterial chemosensory drive, Adv Exp Med Biol 648, 290-297.

11. Samolski, D., Tárrega, J., Antón, A., Mayos, M., Martí,S., Farrero, E., Gűell, R. (2010) Sleep hypoventilation due to increased nocturnal oxygen flow in hypercapnic COPD patients, Respirol 15, 283-288.

12. Nedergaard, M., Kraig, R.P., Tanabe, J., Pulsinelli, W.A. (1991) Dynamics of interstitial and intracellular pH in evolving brain infarct, Am J Physiol 260(3 Pt 2), R581-588.

13. Tregub, P., Kulikov, V., Motin, Y., Bespalov, A., Osipov, I. (2015) Combined exposure to hypercapnia and hypoxia provides its maximum neuroprotective effect during focal ischemic injury in the brain, J Stroke Cerebrovasc Dis 24(2), 381-387. doi: 10.1016/j.jstrokecerebrovasdis.2014.09.003.

14. Andrianopoulos, V., Vanfleteren, L.E., Jarosch, I., Gloeckl, R., Schneeberger, T., Wouters, E.F., Spruit, M.A., Kenn, K. (2016) Transcutaneous carbon-dioxide partial pressure trends during six-minute walk test in patients with very severe COPD, Respir Physiol Neurobiol 233, 52-59. doi: 10.1016/j.resp.2016.08.003. [Epub ahead of print]

15. Perrotti, E., Maroli, M. (1993) Carbon dioxide anesthesia in phlebotomine sand flies (Diptera: Psychodidae): CO2 effect upon two laboratory colonies, J Am Mosq Control Assoc 9(1), 94-96.

16. Vessey, N.Y., Stark, P.M., Flatt, K.L., Bueno, R. Jr. (2008) A multiunit CO2 anesthetizing system for use in transferring mosquitoes during field cage insectcide efficacy tests, J Am Mosq Control Assoc 24(3), 463-464.

17. Caldwell, P.C. (1958) Studies on the internal pH of large muscle and nerve fibres, J Physiol 142(1), 22-62.

18. Badre, N.H., Martin, M.E., Cooper, R.L. (2005) The physiological and behavioral effects of carbon dioxide on Drosophila larvae, Comp Biochem Physiol A 140,363-376.

19. Bierbower, S.M., Cooper, R.L. (2013) The mechanistic action of carbon dioxide on a neural circuit and NMJ communication, $J$ Exp Zool 319A, 340-354.

20. Welsh, M.J., Price, M.P., Xie, J. (2002) Biochemical basis of touch perception: mechanosensory function of degenerin/epithelial $\mathrm{Na}^{+}$channels, J Biol Chem 277(4), 2369-2372.

21. Geffeney, S.L., Goodman, M.B. (2012) How we feel: ion channel partnerships that detect mechanical inputs and give rise to touch and pain perception, Neuron 74(4), 609-619. doi: 10.1016/j.neuron.2012.04.023.

22. Coste, B., Xiao, B., Santos, J.S., Syeda, R., Grandl, J., Spencer, K.S., Kim, S.E., Schmidt, M., Mathur, J., Dubin, A.E., Montal, M., Patapoutian, A. (2012) Piezo proteins are pore-forming subunits of mechanically activated channels, Nature 483(7388), 176-181. doi: 10.1038/nature10812.

23. Ernstrom, G.G., Chalfie, M. (2002) Genetics of sensory mechanotransduction, Annu Rev Genet 36, 411-453.

24. Hsu, P., Haffner, J., Albuquerque, M.L., Leffler, C.W. (1996) pHi in piglet cerebral microvascular endothelial cells: recovery from an acid load, Proc Soc Exp Biol Med 212(3), 256-262.

25. Ritucci, N.A., Chambers-Kersh, L., Dean, J.B., Putnam, R.W. (1998) Intracellular pH regulation in neurons from chemosensitive and nonchemosensitive areas of the medulla, Am J Physiol 275(4 Pt 2), R1152-1163.

26. Alexandrowicz, J.S. (1972) The comparative anatomy of leg propriocetors in some decapod Crustacea, J Mar Biol Assoc UK 52(3), 605-634.

27. Kuffler, S.W. (1954) Mechanisms of activation and motor control of stretch receptors in lobster and crayfish, J Neurophysiol 17, $558-574$.

28. Hartman, H.B., Boettiger, E.G. (1967) The functional organization of the propus-dactylus organ in Cancer irroratus Say, Comp Biochem Physiol 22, 651-663. 
29. Hartman, H.B., Cooper, R.L. (1994) Regeneration and molting effects on a proprioceptor organ in the Dungeness crab, Cancer magister, J Neurobiol 25, 461-471.

30. Cooper, R.L., Hartman, H.B. (1999) Quantification of responses from proprioceptive neurons in the limbs of the crab, Cancer magister, J Exp Zool 284, 629-636.

31. Cooper, R.L. (2008) Mapping proprioceptive neurons on chordotonal organs in the crab, Cancer magister, Crustaceana 81, 447475.

32. Rydqvist, B., Lin, J.H., Sand, P., Swerup, C. (2007) Mechanotransduction and the crayfish stretch receptor, Physiol Behav 92(12), 21-28.

33. Majeed, Z. R., Titlow, J., Hartman, H. B., Cooper, R. (2013) Proprioception and tension receptors in crab limbs: student laboratory exercises, JoVE 80, e51050, doi:10.3791/51050 (2013).

34. Leksrisawat, B., Cooper, A.S., Gilberts, A.B., Cooper, R.L. (2010) Response properties of muscle receptor organs in the crayfish abdomen: A student laboratory exercise in proprioception, JoVE 45, http://www.jove.com/index/details.stp?id=2323 doi:10.3791/2323

35. Baierlein, B., Thurow, A.L., Atwood, H.L., Cooper, R.L. (2011) Membrane potentials, synaptic responses, neuronal circuitry, neuromodulation and muscle histology using the crayfish: Student laboratory exercises, JoVE 47:http://www.jove.com/Details.php?ID=2322 doi: 10.3791/2325.

36. Ryan, J.P., Ryan, H. (1972) The role of intracellular pH in the regulation of cation exchanges in yeast, Biochem J 128(1), 139146.

37. Heming, T.A., Boyarsky, G., Tuazon, D.M., Bidani, A. (1985) pH(i) responses to osmotic cell shrinkage in the presence of open-system buffers, J Appl Physiol 89(4), 1543-1552.

38. Mellergard, P., Siesjo, B.K. (1998) Cerebral energy metabolism and pH, in $p H$ and Brain Function (Kaila K. and Ransom B.R.,Eds.), 67-93, Wiley-Liss, New York.

39. Shrager, P. (1974) Ionic conductance changes in voltage clamped crayfish axons at low pH, J Gen Physiol 64(6), 666-690.

40. Meves, H., Volkner, K.G. (1958) Effect of CO2 on resting membrane potential and electric constants of striated muscle fibers, Pflugers Arch. 1265(5), 457-476.

41. Somjen, G.G., Tombaugh, G.C. (1998) pH modulation of neuronal excitability and central nervous system functions, in $p H$ and Brain Function, (Kaila K. and Ransom B.R., Eds.), 373-394, Wiley-Liss, New York.

42. Roos, A., Boron, W.F. (1981) Intracellular pH, Physiol Rev 61(2), 296-434.

43. Thomas, R.C. (1984) Experimental displacement of intracellular $\mathrm{pH}$ and the mechanism of its subsequent recovery, $J$ Physiol 354, 3-22.

44. Thomas, R.C., Schwiening, C.J. (1998) Intracelluar pH regulation in invertebrate neurons, in pH and Brain Function, (Kaila, K. and Ransom, B.R., Eds.), 195-209, Wiley-Liss, New York.

45. Hazel, J.R., McKinley, S.J., Williams, E.E. (1992) Thermal adaptation in biological membranes: interacting effects of temperature and $\mathrm{pH}, J$ Comp Physiol B 162(7), 593-601.

46. Cooper, R.L., Ward, E., Braxton, R., Li, H., Warren, W.M. (2003) The effects of serotonin and ecdysone on primary sensory neurons in crayfish, Microsc Res Techn 60, 336-345.

47. Pasztor, V.M., MacMillan, D.L. (1990) The actions of proctolin, octopamine and serotonin on the crustacean proprioceptors show species and neurone specificity, J Exp Biol 152, 485-504.

48. Peetz, W., Winter, C. (1980) Alteration of the excitation state in receptor cells by hemolymph: A new phenomenon in the crayfish stretch receptor, Comp Biochem Physiol (A) 67, 59-68.

49. Begg, M., Cruickshank, W.J. (1963) A partial analysis of Drosophila larval haemolymph, Proc, R Soc Edinb B 58, $215-36$.

50. Stewart, B.A., Atwood, H.L., Renger, J.J., Wang, J., Wu, C.F. (1994) Improved stability of Drosophila larval neuromuscular preparation in haemolymph-like physiological solutions, J Comp Physiol A 175, 179-191.

51. deCastro, C., Titlow, J., Majeed, Z.R., Cooper, R.L. (2014) Analysis of various physiological salines for heart rate, CNS function, and synaptic transmission at neuromuscular junctions in Drosophila melanogaster larvae, J Comp Physiol A 200, 8392.

52. Traynelis, S.F. (1998) pH modulation of ligand gated ion channels, in $p H$ and Brain Function, (Kaila, K. and Ransom, B.R., Eds), 395-446, Wiley-Liss, New York.

53. Traynelis, S.F., Cull-Candy, S.G. (1991) Pharmacological properties and $\mathrm{H}^{+}$sensitivity of excitatory amino acid receptor channels in rat cerebellar granule neurons, J Physiol 433, 727-763.

54. Wu, X., Christensen, B.N. (1996) Proton inhibition of the NMDA-gated channel in isolated catfish cone horizontal cells, Vision Res 36(11), 1521-1528.

55. Taira, T., Smirnov, S., Voipio, J., Kaila, K. (1993) Intrinsic proton modulation of excitatory transmission in rat hippocampal slices, Neuroreport 4(1), 93-96.

56. Ballanyi, K., Kaila, K (1998) Transmitter-evoked shifts in intracellular pH, in $p H$ and Brain Function, (Kaila K. and Ransom B.R., Eds.), 291-308, Wiley-Liss, New York. 
57. Voipo, J. (1998) Diffusion and buffering aspects of $\mathrm{H}^{+}, \mathrm{HCO} 3-$, and CO2 movments in brain tissue,.in $p H$ and Brain Function, (Kaila K. and Ransom B.R., Eds.), 45-66, Wiley-Liss, New York.

58. Hartings, J.A., Shuttleworth, C.W., Kirov, S.A., Ayata, C., Hinzman, J.M., Foreman, B., Andrew, R.D., Boutelle, M.G., Brennan, K.C., Carlson, A.P., Dahlem, M.A., Drenckhahn, C., Dohmen, C., Fabricius, M., Farkas, E., Feuerstein, D., Graf, R., Helbok, R., Lauritzen, M., Major, S., Oliveira-Ferreira, A.I., Richter, F., Rosenthal, E.S., Sakowitz, O.W., Sánchez-Porras, R., Santos, E., Schöll, M., Strong, A.J., Urbach, A., Westover, M.B., Winkler, M.K., Witte, O.W., Woitzik, J., Dreier, J.P. (2016) The continuum of spreading depolarizations in acute cortical lesion development: Examining Leão's legacy, J Cereb Blood Flow Metab pii: 0271678X16654495. PMID: 27328690

59. Appavu, B., Vanatta, L., Condie, J., Kerrigan, J.F., Jarrar, R. (2016) Ketogenic diet treatment for pediatric super-refractory status epilepticus, Seizure 41, 62-65. doi: 10.1016/j.seizure.2016.07.006. [Epub ahead of print] PMID:27475280

60. Burakgazi, E., French, J.A. (2016) Treatment of epilepsy in adults, Epileptic Disord 18(3), 228-239. doi: 10.1684/epd.2016.0836. PMID: 27435035

61. van der Louw, E., van den Hurk, D., Neal, E., Leiendecker, B., Fitzsimmon, G., Dority, L., Thompson, L., Marchió, M., Dudzińska, M., Dressler, A., Klepper, J., Auvin, S., Cross, J.H. (2016) Ketogenic diet guidelines for infants with refractory epilepsy, Eur J Paediatr Neurol Jul 17. pii: S1090-3798(16)30098-8. doi: 10.1016/j.ejpn.2016.07.009. [Epub ahead of print]

62. Kaila, K., Ransom, B.R. (1998) Concept of $\mathrm{pH}$ and its importance in neurobiology, in $p H$ and Brain Function, (Kaila K. and Ransom B.R., Eds.), 3-10, Wiley-Liss, New York.

63. Dreier, J.P., Fabricius, M., Ayata, C., Sakowitz, O.W., Shuttleworth, C.W., Dohmen, C., Graf, R., Vajkoczy, P., Helbok, R., Suzuki, M., Schiefecker, A.J., Major, S., Winkler, M.K., Kang, E.J., Milakara, D., Oliveira-Ferreira, A.I., Reiffurth, C., Revankar, G.S., Sugimoto, K., Dengler, N.F., Hecht, N., Foreman, B., Feyen, B., Kondziella, D., Friberg. C.K., Piilgaard, H., Rosenthal, E.S., Westover, M.B., Maslarova, A., Santos, E., Hertle, D., Sánchez-Porras, R., Jewell, S.L., Balança, B., Platz, J., Hinzman, J.M., Lückl, J., Schoknecht, K., Schöll, M., Drenckhahn, C., Feuerstein, D., Eriksen, N., Horst, V., Bretz, J.S., Jahnke, P., Scheel, M., Bohner, G., Rostrup, E., Pakkenberg, B., Heinemann, U., Claassen, J., Carlson, A.P., Kowoll, C.M., Lublinsky, S., Chassidim, Y., Shelef, I., Friedman, A., Brinker, G., Reiner, M., Kirov, S.A., Andrew, R.D., Farkas, E., Güresir, E., Vatter, H., Chung, L.S., Brennan, K.C., Lieutaud, T., Marinesco, S., Maas, A.I., Sahuquillo, J., Dahlem, M.A., Richter, F., Herreras, O., Boutelle, M.G., Okonkwo, D.O., Bullock, M.R., Witte, O.W., Martus, P., van den Maagdenberg, A.M., Ferrari, M.D., Dijkhuizen, R.M., Shutter, L.A., Andaluz, N., Schulte, A.P., MacVicar, B., Watanabe, T., Woitzik, J., Lauritzen, M., Strong, A.J., Hartings, J.A. (2016) Recording, analysis, and interpretation of spreading depolarizations in neurointensive care: Review and recommendations of the COSBID research group, J Cereb Blood Flow Metab pii: 0271678X16654496. [Epub ahead of print] Review. PMID: 27317657

64. Astrup J, Symon L, Branston NM, Lassen NA. (1977) Cortical evoked potential and extracellular $\mathrm{K}^{+}$and $\mathrm{H}^{+}$at critical levels of brain ischemia, Stroke 8(1):51-57.

65. Dayaram,V., Malloy, C., Martha, S., Alvarez, B., Chukwudolue, I., Dabbain, N., D.mahmood, D., Goleva, S., Hickey, T., Ho, A., King, M., Kington, P., Mattingly, M., Potter, S., Simpson, L., Spence, A., Uradu, H., Van Doorn, J.L., and Cooper, R.L. (2017). Stretch activated channels in proprioceptive chordotonal organs of crab and crayfish are sensitive to Gd3 $3^{+}$but not amiloride, ruthenium red or low pH. IMPLUSE https://impulse.appstate.edu/issues/2017

66. Malloy, C., Dayaram,V., Martha, S., Alvarez, B., Chukwudolue, I., Dabbain, N., D.mahmood, D., Goleva, S., Hickey, T., Ho, A., King, M., Kington, P., Mattingly, M., Potter, S., Simpson, L., Spence, A., Uradu, H., Van Doorn, J.L., Weineck, K. and Cooper, R.L. (2017). The effects of potassium and muscle homogenate on proprioceptive responses in crayfish and crab. $J$ Exp Zool (00)1-14. (early view) https://doi.org/10.1002/jez.2096

\section{ABOUT THE STUDENT AUTHORS}

Many of the authors were students in a neurophysiology lab-based class addressing authentic scientific based questions in regard to the topic of examining how extracellular and intracellular $\mathrm{pH}$ would influence proprioception. Most of the undergraduate students have now graduated with a BS in biology or neuroscience.

\section{PRESS SUMMARY}

Two invertebrate model preparations are used to demonstrate how external and internal cellular $\mathrm{pH}$ changes as well as exposure to $\mathrm{CO}_{2}$ can alter neuronal function for proprioception. Proprioceptive neurons are most affected when intracellular $\mathrm{pH}$ is decreased by exposure to propionic acid or saline containing $\mathrm{CO}_{2}$. The ease in recording from these neurons can help researchers understanding mechanistic properties of mechanosensitive receptors in other organisms, such as muscle spindles in skeletal muscles of mammals and tactical as well as pressure (i.e., blood pressure) sensory receptors. 


\title{
Derivation of Explicit Solutions Describing Early Stages of Platelet Activation
}

\author{
Rachel Austin, Scott Fones*, Dominic Santoleri, Kaitlyn Thomesen, Pak-Wing Fok \\ Department of Mathematical Sciences, University of Delaware, Nerwark, DE \\ Students: rraustin@udel.edu,*sfones@udel.edu,dsantole@udel.edu, thomesen@udel.edu \\ Mentor: pakwing@udel.edu
}

\begin{abstract}
The formation of blood clots is vital for biological repair of injured blood vessels. When a blood vessel is injured, platelets come into contact with collagen, causing glycoprotein VI (GPVI) to undergo a conformational change and initialize the clotting process. This project aimed to simplify and solve a system of coupled ordinary differential equations (ODEs) proposed in Model A of Regulation of Early Steps of GPVI Signal Transduction by Phosphatase: A Systems Biology Approach by JL Dunster et al., modeling early platelet activation kinetics. In doing so, the ODEs were non-dimensionalized and the approximate analytical solutions were then found. The approximate solutions compare favorably to the numerical solutions and provide deeper insight into the signal regulation pathway. Most notably, the solutions expose a time at which the pathway dynamics change drastically. This illustrates the critical role of cytosolic spleen tyrosine kinase (Syk) as a molecular timer in the cascade.
\end{abstract}

\section{KEYWORDS}

Platelet Activation; Signal Cascade; Coupled Differential Equations; Asymptotic Analysis; Systems Biology; Mathematical Modeling; Model Simplification; Glycoprotein VI; Spleen Tyrosine Kinase; Syk Activation Dynamics

\section{INTRODUCTION}

Blood is composed mostly of plasma, white blood cells, red blood cells, and platelets. Platelets are anuclear, biconcave discs responsible for the detection and repair of structural damage to the blood vessel wall. The extracellular matrix, ECM, surrounding blood vessels is composed mainly of collagen. When the blood vessel wall is compromised, the platelets inside the blood vessels are exposed to the ECM (Figure 1a). The platelets respond to the presence of collagen by initiating a repair pathway. ${ }^{1}$ More specifically, the interactions with glycoprotein VI (GPVI), cause the platelet to undergo a conformational change that initiates the clotting process (Figure 1b). This conformational change starts a signaling cascade that relies heavily on the phosphorylation of secondary messengers to amplify the signal. This helps to aggregate additional platelets to the wound, and allows clot formation to occur. Once the clot begins to form, regulatory feedback loops within this signaling cascade modulate the strength and duration of the signal. Although the forward signaling cascade has been well studied, little information exists on regulation of the signal. Evidence indicates that as the signaling process occurs, products further downstream interact with earlier events to regulate and modify the strength and duration of the clotting response. ${ }^{1}$

The inappropriate activation of clot formation can lead to thrombosis, heart disease, and stroke, all of which are potentially fatal. ${ }^{3}$ Developing a model to depict this signaling pathway can lead to a better quantitative understanding of clotting. Through mathematical modeling, physicians can better detect and target crucial reactions in this pathway. These models could potentially aide in the development of medication that prevents inappropriate clot formation, making treatment more effective. In addition, spleen tyrosine kinase, Syk, plays a significant role in immune cell signaling. ${ }^{4}$ This role may be better understood though modeling Syk's dynamics in the context of platelet activation. 
Three models for the initial steps of the signal cascade for platelet activation were developed by Dunster et al. ${ }^{1}$ Their simplest model, Model A, proposes seven differential equations correlating to the different steps and participants in the cascade. These equations are useful in developing a model, but the information obtained from a system of differential equations is limited. Finding analytic approximations for these equations can provide more insight into the model, such as the concentration of each component of the cascade at a given point in time.

After highlighting the importance and current understanding of this pathway in section 3, we introduce a model proposed by Dunster et al. in section 4.1. In section 5.1, these equations are non-dimensionalized to obtain unitless constants that can be compared directly. We proceeded to simplify and solve the seven differential equations to obtain solutions in section 5.6. In section 6, we offer conclusions and insights provided by the analytical solutions.

\section{Literature Review}

Dunster et al. have used their understanding of the clotting cascade pathway to develop a model of the signaling pathway. ${ }^{1}$ However, the authors concede that the negative feedback loops are not well understood. Other mathematical models for platelet signaling tend to focus on signal activation and have been remarkably successful in predicting calcium regulation in platelets during signal transduction. ${ }^{5}$ These models typically focus on forward signal transduction while signal regulation mechanisms remain largely unstudied. These feedback inhibition pathways are crucial for understanding the regulation of this pathway and the steady state level for phosphorylated spleen tyrosine kinase, Syk. Several potential feedback processes are considered and incorporated in later, more complex modeling systems by Dunster et al. ${ }^{1}$ Such a feedback loop was investigated, with particuar emphasis placed on the question of whether the ligand T-cell ubiquitin ligand-2, TULA-2, is partially responsible for the regulation of phosphorylated Syk. ${ }^{6}$ A negative correlation between the concentration of TULA2 and activated Syk levels was discovered, in addition to an apparent specificity of TULA-2 for Syk. Not only is TULA-2 suspected to negatively regulate activated Syk, but c-Cbl, a kinase from the Src family that is activated along the clotting signal cascade may also be involved. Studies have also indicated a potential regulatory role that c-Cbl has on activated Syk. ${ }^{7}$ These feedback loops are incorporated into the models from the Dunster et al. paper to predict activated Syk concentrations. The incorporation of these loops is further supported by the key role Src family kinases play in initiating platelet activation. ${ }^{8}$ The regulatory role of phosphatases can be considered in modulating the cellular response to signaling. ${ }^{9}$ Phosphatases also play a regulatory role in the activation of Syk and the maintenance of the clotting signal.

To further support the many checkpoints and feedback loops in this physiological phenomenon, the idea of Syk being the main checkpoint for this cascade has been studied. ${ }^{10}$ Knowing that Syk activation is heavily regulated supports the assumption that Dunster made when focusing on the concentration of activated Syk as an indicator of the clotting signal progression. The Y525 motif on Syk, which is the location where a tyrosine residue becomes phosphorylated during Syk activation, is important for regulation and activation. ${ }^{10}$ ITAM-Syk binding and later Syk activation heavily relies on this motif. Most of the feedback mechanisms, such as phosphatase dephosphorylation of Syk, depend on protein recognition of Y525.

The mechanisms behind the GPVI activation are also vital to the understanding of the clotting signaling cascade. ${ }^{2}$ Dunster et al.'s mathematical model of platelet activation uses this to explain the high affinity of Syk for ITAM on the GPVI receptor, leading to Syk strongly binding to the phosphorylated receptor complex. Additional research explores platelet activation and blood coagulation as complementary processes, illustrating the importance of regulatory processes in ensuring efficient responses to injury. ${ }^{11}$

Understanding the complex mechanisms of platelet activation allows for more effective antithrombotic medicines targeting the prevention of errant clot formation. Arterial thrombosis is an acute complication that forms on chronic lesions of atherosclerosis and results in heart attacks and stroke. ${ }^{3}$ Further exploration of platelet activation will lead to a better understanding of the formation and progression of atherosclerotic plaque. As the most common cause of mortality in developed countries, there are large clinical implications to a deeper understanding of clotting dynamics. ${ }^{1,3}$ Beyond atherosclerosis, 
platelet activation contributes to the long-term recovery outcomes of heart transplantation. ${ }^{12}$ Long-term survivors of heart transplantation show increased activation of platelets, potentially contributing to increased immune activation and complications. ${ }^{3}$ As a component of endothelial dysfunction, the inverse relationship between platelet activation and endotheliumdependent vasomotor function appears to explain the connection between vasomotor dysfunction and atherothrombotic events. ${ }^{13}$ These phenomena, along with other connections between platelet activation and chronic illness, illustrate the importance of understanding the role of platelet activation.

\section{GOVERNING EQUATIONS}

Description of Dunster's Model A

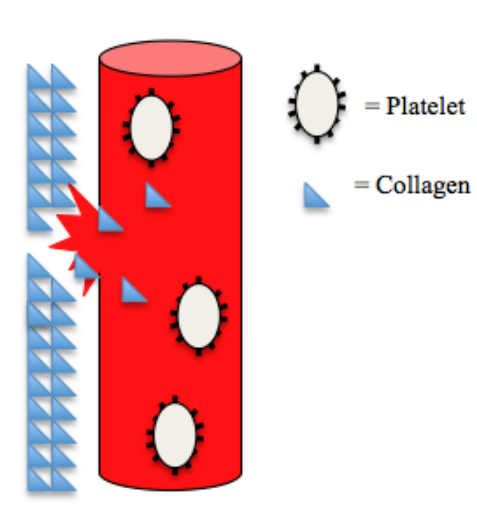

(a)

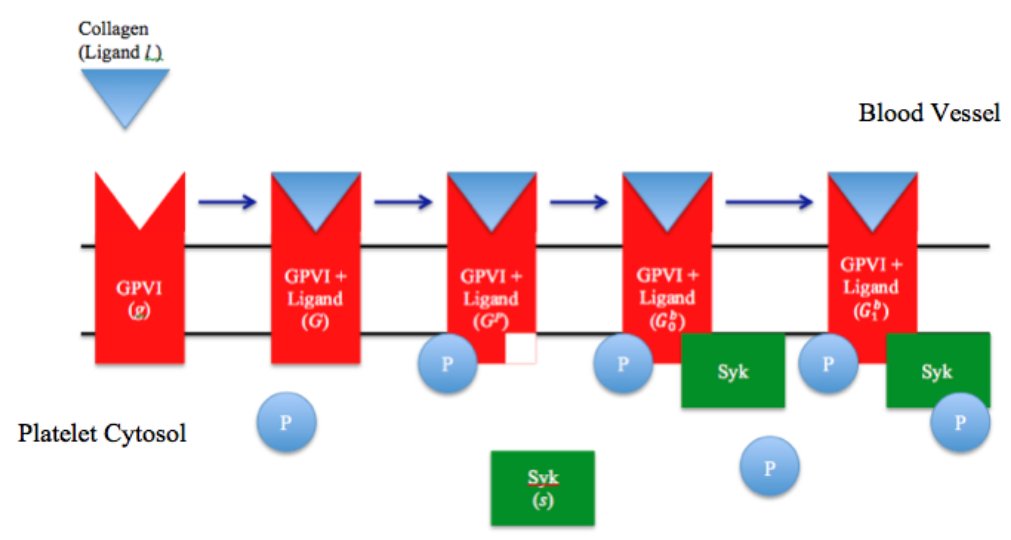

(b)

Figure 1. (a) Damage to the blood vessel wall exposes platelets to collagen. Other blood vessel components (red blood cells, white blood cells, etc. have been excluded for simplicity. (b) Exposure of GPVI at the platelet surface to collagen begins the platelet activation cascade by forming a receptor complex. The receptor complex is then phosphorylated and Syk binds to the complex, activating the receptor and initiating downstream signaling.

This paper analyzes the intermediates in Model A of the platelet activation signaling pathway developed by Dunster et al. ${ }^{1}$ Collagen normally exists outside of the blood vessel, but upon damage to the blood vessel, collagen gets exposed to the bloodstream and platelets (Figure 1a). Collagen or the multivalent GPVI-selective agonist, collagen-related peptide (CRP), used during the experiments (both denoted as $l$ ) exposed to the platelets in the extracellular matrix can then interact with GPVI at the cell surface. As the ligand reversibly binds to the free GPVI receptor, $g$, on the cell membrane surface, the receptor-ligand complex, $G$, forms. The reverse reaction then involves the dissociation of $G$ back into $l$ and $g$. Following the formation of the receptor-ligand complex, it becomes phosphorylated, $G^{p}$. This phosphorylation allows for recruitment and activation of the cytosolic protein-tyrosine kinase, Syk, found inside the platelet cytosol. Syk, denoted $s$, binds to the phosphorylated receptor complex, $G^{p}$, to form $G_{0}^{b}$. Since GPVI associates with the ITAMs, when Syk aggregates and interacts with the activated ITAM, its tyrosine 525 residue (Y525) gets phosphorylated $\left(G_{1}^{b}\right)$. This entire process, resulting in the phosphorylation of Syk, initiates the downstream signaling. The high binding affinity of Syk to the ITAM occurs through two bound tyrosine residues, resulting in protection of the ITAM from dephosphorylation. The receptor-ligand complex formation and the phosphorylation of Syk steps are reversible and have the potential for being regulated by outside enzyme or downstream products. Figure $1 \mathrm{~b}$ demonstrates the mechanism described above, and Table 1 and Table 2 detail the variables and parameters involved for this cascade along with the values for each, respectively.

The rate at which each reactant, intermediate, and product in the mechanism shown in Figure $1 \mathbf{b}$ is created or consumed can be modeled by the seven differential equations determined by Dunster et al. (Equations (1a-1g)). ${ }^{1}$ Equations $1 \mathrm{a}$ and $1 \mathbf{b}$ are very similar and measure the rates of ligand and GPVI consumption $\left(\frac{d l}{d t}=-\frac{k_{1}}{V_{e} A_{v}} g l+\frac{k_{-1}}{V_{e} A_{v}} G\right.$, for $l$ and $\frac{d g}{d t}=-k_{1} g l+k_{-1} G$ for $g$ ). The first term in these equation demonstrates the consumption of $l$ and $g$ through their association to create $G$ while the second term represents the reverse reaction where $G$ dissociates back into $l$ and $g$. Because $g$ is fixed to the platelet 
membrane, it can only interact with $l$ when it is close to the membrane. As such, the ligand rate equation, Equation 1a, is scaled down by the volume of the extracellular matrix $\left(V_{e}\right)$ so that only the ligand molecules close to the platelet membrane are accounted for in the equation. Additionally, Equation 1a is divided by Avogadro's Number $\left(A_{v}\right)$ to convert the mM units $\left(\frac{\text { moles }}{\mathrm{m}^{3}}\right)$ of $l$ to molecules like $g$.

The first two terms of Equation 1c are the opposite of those in Equations 1a and 1b. These terms represent the same forward and reverse reaction involving ligand-receptor binding, but presented from the alternate view. The direction that consumes $l$ and $g$ creates $G$ and the direction that creates $l$ and $g$ consumes $G$, hence the opposite signs. The final term in Equation 1c accounts for the rate that $G$ is consumed in the next step of the pathway as it gets phosphorylated to $G^{p}$. Together, these terms outline how quickly $G$ is created at the beginning of the reaction and then consumed in both forward and reverse reactions $\left(\frac{d G}{d t}=k_{1} g l-k_{-1} G-k_{2} G\right)$.

$G^{p}$ creation depends on $G$ consumption. Hence the first term of Equation $1 \mathbf{d}\left(\frac{d G^{p}}{d t}=k_{2} G-\frac{k_{3}}{V_{p} A_{v}} G^{p} s\right)$ is the opposite of the last term of Equation 1c. The dephosphorylation of $G$ is not significantly reversible and so there is no term denoting the reverse of this reaction. This is due to the speed with which the next step of the pathway occurs. $G^{p}$ interacts with $s$ from the cytosol to create $G_{0}^{b}$. This reaction creates the second term in Equation $1 \mathrm{~d}$ and the rate equation for $s$, Equation 1e $\left(\frac{d s}{d t}=-\frac{k_{3}}{V_{p} A_{v}} G^{p} s\right)$ which only participates in this reaction within the pathway. Because $s$ exists in the cytosol similarly to how $l$ exists in the extracellular matrix, this term has to be adjusted to account for the fact that this interaction can only occur along the membrane. However, since $s$ is cytosolic instead of extracellular, the volume of the platelet $\left(V_{p}\right)$ is used in the calculation instead of the external environment. Additionally, the rate constant for this step of the reaction $\left(k_{3}\right)$ is so large that the reaction drives forward, consuming $G^{p}$ as quickly as it is created.

The last two equations represent the final part of this pathway. $G_{0}^{b}$, representing the ligand-bound receptor interacting with Syk, gets phosphorylated to create $G_{1}^{b}$ which can further initiate downstream signaling. The first term of Equation If $\left(\frac{d G_{0}^{b}}{d t}=\frac{k_{3}}{V_{p} A_{v}} G^{p} s-\rho_{1} G_{0}^{b}+\gamma_{1} G_{1}^{b}\right)$ shows the irreversible creation of $G_{0}^{b}$ while the last two terms show the forward and reverse rates of its reversible phosphorylation into $G_{1}^{b}$. Similarly, Equation $\lg \left(\frac{d G_{1}^{b}}{d t}=\rho_{1} G_{0}^{b}-\gamma_{1} G_{1}^{b}\right)$ shows the same forward and reverse reactions but in the opposite direction. As such, these two protein forms eventually reach an equilibrium once all of $s$ is consumed by the previous reaction.

\begin{tabular}{|c|c|c|c|}
\hline Variable & Description & Units & Initial Value \\
\hline$l$ & Ligand & $\frac{\text { moles }}{\mathrm{m}^{3}}(\mathrm{mM})$ & $3 \times 10^{-2}$ \\
\hline$t$ & Time & seconds & 0 \\
\hline$g$ & GPVI & molecules & 5000 \\
\hline$s$ & Cytosolic Syk & molecules & 2763 \\
\hline$G$ & GPVI-Ligand Complex & molecules & 0 \\
\hline$G^{p}$ & Phosphorylated Receptor & molecules & 0 \\
\hline$G_{0}^{b}$ & Syk-Receptor Complex & molecules & 0 \\
\hline$G_{1}^{b}$ & Syk with Y525 Phosphorylated & molecules & 0 \\
\hline
\end{tabular}

Table 1. Variables used in the signaling model. Initial concentrations of GPVI and cytosolic Syk were determined with immunoblotting. During experiments, the collagen-related peptide used as the ligand was always maintained at high saturation levels and so a non-rate limiting initial value was selected. ${ }^{1}$ 


\begin{tabular}{|c|c|c|c|}
\hline Parameter & Description & Units & Value \\
\hline$k_{1}$ & Rate of Ligand Binding & $\frac{\mathrm{m}^{3}}{\mathrm{moles} \cdot \mathrm{s}}$ & 8 \\
\hline$k_{-1}$ & Rate of Ligand Dissociation & $\mathrm{s}^{-1}$ & $3.02 \times 10^{-2}$ \\
\hline$V_{e}$ & Extracellular Volume per Cell & $\mathrm{m}^{3}$ & $3.3 \times 10^{-9}$ \\
\hline$V_{p}$ & Platelet Volume & $\mathrm{m}^{3}$ & $7.4 \times 10^{-18}$ \\
\hline$k_{2}$ & Rate of Receptor Phosphorylation & $\mathrm{s}^{-1}$ & $3.02 \times 10^{-2}$ \\
\hline$k_{3}$ & Rate Syk Binds to Receptor & $\mathrm{s}^{-1}$ & $9.55 \times 10^{5}$ \\
\hline$\rho_{1}$ & Rate of Syk Phosphorylation & $\mathrm{s}^{-1}$ & $5.13 \times 10^{-1}$ \\
\hline$\gamma_{1}$ & Rate of Syk Dephosphorylation & $\mathrm{s}^{-1}$ & 3.53 \\
\hline$T$ & Experimental Duration & $\mathrm{s}$ & 250 \\
\hline
\end{tabular}

Table 2. Parameters used in the model Equations $1 \mathrm{a}-1 \mathrm{~g}$, and in their non-dimensionalization Equations $2 \mathrm{a}-2 \mathrm{~g}$. $k_{1}$ and $k_{-1}$, the rate constants for ligand association and dissociation, were previously reported. ${ }^{16}$ The other rate constants were determined through parameter fitting using estimates based on the kinetics of similar proteins or of proteins in different cell lines. Platelet volume was determined during sample collection and the extracellular volume was calculated from there. ${ }^{1}$

In summary, the set of ODEs given by Dunster et al. ${ }^{1}$ to represent the initial steps of platelet activation are below.

$$
\begin{aligned}
\frac{d l}{d t} & =-\frac{k_{1}}{V_{e} A_{v}} g l+\frac{k_{-1}}{V_{e} A_{v}} G \\
\frac{d g}{d t} & =-k_{1} g l+k_{-1} G \\
\frac{d G}{d t} & =k_{1} g l-k_{-1} G-k_{2} G \\
\frac{d G^{p}}{d t} & =k_{2} G-\frac{k_{3}}{V_{p} A_{v}} G^{p} s \\
\frac{d s}{d t} & =-\frac{k_{3}}{V_{p} A_{v}} G^{p} s \\
\frac{d G_{0}^{b}}{d t} & =\frac{k_{3}}{V_{p} A_{v}} G^{p} s-\rho_{1} G_{0}^{b}+\gamma_{1} G_{1}^{b} \\
\frac{d G_{1}^{b}}{d t} & =\rho_{1} G_{0}^{b}-\gamma_{1} G_{1}^{b}
\end{aligned}
$$

Equation 1a.

Equation $1 b$.

Equation 1c.

Equation 1d.

Equation 1e.

Equation 1f.

Equation 1g.

Definitions of all variables and parameters are given in Tables 1 and 2, respectively.

This system of equations can be solved numerically (Figure 2), allowing relative concentrations to be compared visually throughout the signaling cascade. This approach also shows the approximate times at which different stages of the cascade are switched on or off and when steady state is reached. Subsequent decomposition and simplification of this system can be compared to these numerical solutions for validation.

\section{Formulation of Assumptions}

The clotting signal cascade is a complex system with a myriad of enzymes, secondary messengers, and ligands that work to regulate its activation and steady state. This model operates under several assumptions, ${ }^{1}$ and only considers the activation of GPVI and the phosphorylation of Syk. The following assumptions impose spatial uniformity on the system, while granting the ability to describe it via mass action kinetics. Other simplifications made in this model include: no feedback mechanisms, a single phosphorylation site, all or nothing responses, and single pathway responses. This simplifies the underlying biological mechanisms of the clotting cascade, but allows us to obtain explicit solutions for all the reactants in the system. 


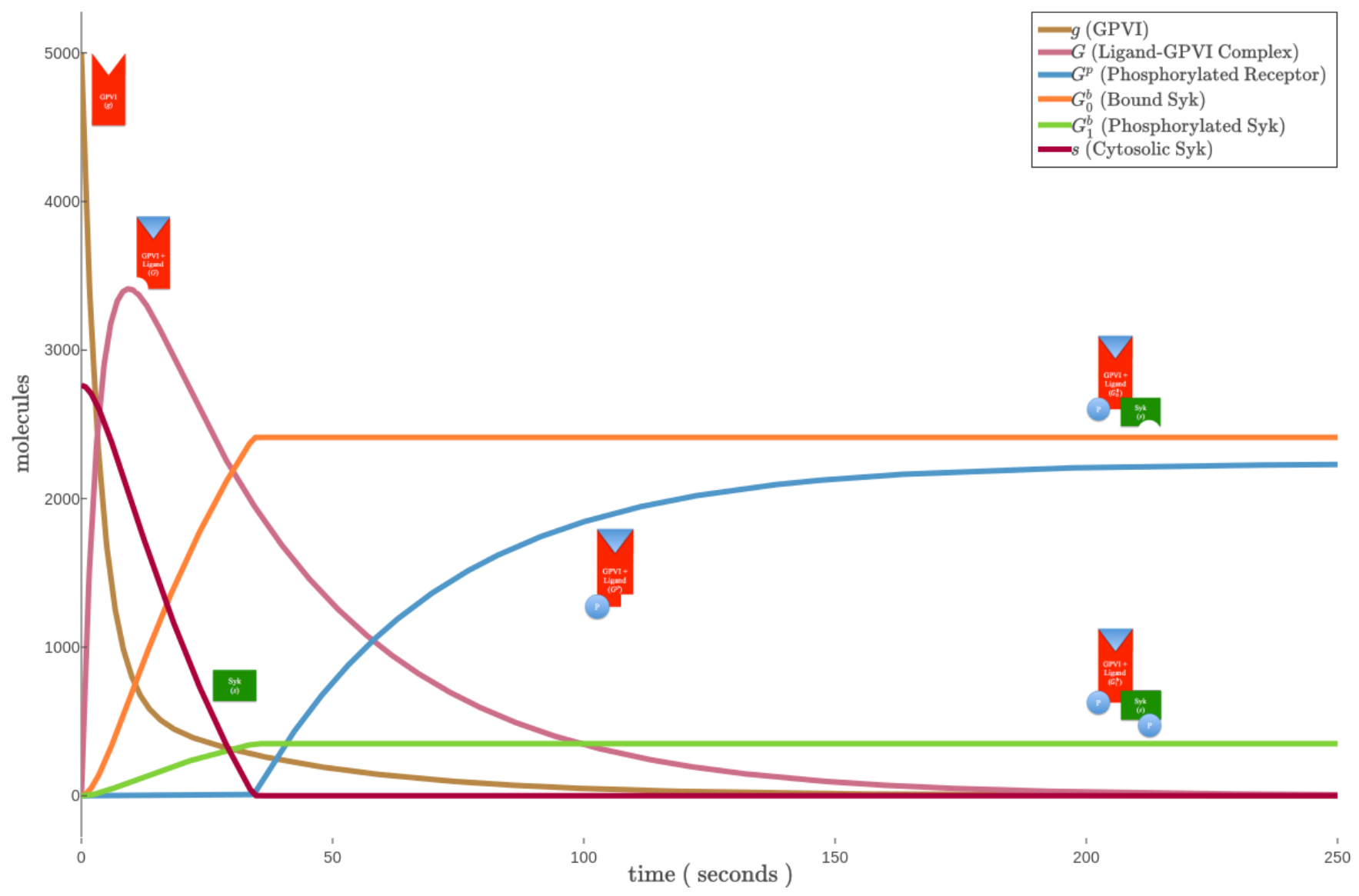

Figure 2. Numerical Solutions to Signaling Cascade. The solutions to the system of ODEs (Equations $1 \mathrm{~b}$ through $1 \mathrm{~g}$ ) with initial conditions and parameters as detailed in Tables 1 and 2. The proteins present during the start of the reaction $(g$ and $s)$ get consumed as the reaction proceeds and go to 0 . The intermediate, $\mathrm{G}$, is initially created, but as the reaction proceeds gets consumed. $G^{p}$ gets created, but its reaction with $s$ is so fast that it is immediately converted into $G_{0}^{b}$ which can reversibly be phophorylated into $G_{1}^{b}$. Once all of $s$ is consumed, no new $G_{0}^{b}$ can be made so $G^{p}$ begins to accumulate and $G_{0}^{b}$ and $G_{1}^{b}$ reach an equilibrium.

Two important assumptions for ODE formulation are:

Assumption 1: The platelet's surface area and receptor density is constant.

Justification: Anuclear cells, such as platelets, lack the requisite genetic material for the production of additional cellular components. This holds for the proteins, phospholipids, or cholesterols that would need to be incorporated into the cellular membrane.

Assumption 2: Cytosolic, membrane, and extracellular components are evenly distributed.

Justification: Rapid diffusion leads to an even dispersal of proteins within the platelet and on its surface. This implies that spatial variation is irrelevant. Further, the platelet's conformational change, induced by the cascade, occurs on a larger timescale than the initial signaling phenomenon of interest.

It is important to note that this model is the most simple model offered by Dunster et al. ${ }^{1}$ It ignores the feedback mechanisms and increased biological fidelity of later models. 


\section{MATHEMATICAL APPROXIMATION}

\section{Non-Dimensionalization}

The system of ODEs is non-dimensionalized. In addition to reducing the number of parameters, this provides a means to compare the rates of each reaction step of platelet signaling activation, which is shown in Figure 2. This helps to determine which kinetic steps are relatively fast or slow in comparison to the other steps. Table 3 presents the non-dimensionalized parameters, variables and their definitions in terms of the original, dimensional parameters.

The dimensionless ODEs are as follows.

$$
\begin{aligned}
\frac{d \Gamma}{d \tau} & =-\hat{\alpha}_{1} \varphi \Gamma+\alpha_{-1} \Phi \\
\frac{d \varphi}{d \tau} & =-\hat{\alpha}_{1} \varphi \Gamma+\alpha_{-1} \Phi \\
\frac{d \Phi}{d \tau} & =\hat{\alpha}_{1} \varphi \Gamma-\alpha_{-1} \Phi-\beta \Phi \\
\frac{d \Phi^{p}}{d \tau} & =\beta \Phi-\eta \Phi^{p} \sigma \\
\frac{d \sigma}{d \tau} & =-\eta \Phi^{p} \sigma \\
\frac{d \Phi_{0}^{b}}{d \tau} & =\eta \Phi^{p} \sigma-\epsilon \Phi_{0}^{b}+\theta \Phi_{1}^{b} \\
\frac{d \Phi_{1}^{b}}{d \tau} & =\epsilon \Phi_{0}^{b}-\theta \Phi_{1}^{b}
\end{aligned}
$$

Equation 2a.

Equation 2b.

Equation 2c.

Equation 2d.

Equation 2e.

Equation 2f.

Equation 2g.

\begin{tabular}{|c|c|c|}
\hline Variable & Definition & Initial Value \\
\hline$\tau$ & $\frac{t}{T}$ & 0 \\
\hline$\Gamma$ & $\frac{l V_{e} A_{v}}{g_{0}}$ & $1.2 \times 10^{12}$ \\
\hline$\varphi$ & $\frac{g}{g_{0}}$ & 1 \\
\hline$\sigma$ & $\frac{s}{g_{0}}$ & 0.55 \\
\hline$\Phi$ & $\frac{G}{g_{0}}$ & 0 \\
\hline$\Phi^{p}$ & $\frac{G^{p}}{g_{0}}$ & 0 \\
\hline$\Phi_{0}^{b}$ & $\frac{G_{0}^{b}}{g_{0}}$ & 0 \\
\hline$\Phi_{1}^{b}$ & $\frac{G_{1}^{b}}{g_{0}}$ & 0 \\
\hline
\end{tabular}

(a)

\begin{tabular}{|c|c|c|}
\hline Parameter & Definition & Value \\
\hline$\hat{\alpha}_{1}$ & $\frac{k_{1} T g_{0}}{V_{e} A_{v}}$ & $5.03 \times 10^{-9}$ \\
\hline$\alpha_{1}$ & $k_{1} T l_{0}$ & 60 \\
\hline$\alpha_{-1}$ & $k_{-1} T$ & 8 \\
\hline$\beta$ & $k_{2} T$ & 8 \\
\hline$\eta$ & $\frac{k_{3}}{V_{p} A_{v}} T g_{0}$ & $2.68 \times 10^{5}$ \\
\hline$\epsilon$ & $\rho_{1} T$ & 128 \\
\hline$\theta$ & $\gamma_{1} T$ & 882 \\
\hline
\end{tabular}

(b)

Table 3. Components of the Non-Dimensional System of ODEs. These tables represent the dimensionless variables (a) and parameters (b) from Dunster et al. ${ }^{1}$ Their corresponding values can be found in Table 1 and Table 2, respectively.

A similar labeling system to the dimensional equations, Equations $1 \mathrm{a}-1 \mathrm{~g}$, is used to allow for easier comparison of the corresponding equations. The dimensionless equations are presented in the order that the reactions shown in Figure 1 occur. Equations $2 \mathrm{a}-2 \mathrm{~g}$ can also be written as a series of chemical reactions. This helps illustrate the approach taken to solve each step.

$$
\Gamma+\varphi \frac{\hat{\alpha}_{1}}{\hat{\alpha}_{-1}} \Phi \stackrel{\beta}{\longrightarrow} \Phi^{p} \underset{\eta}{\stackrel{\sigma}{\longrightarrow}} \Phi_{0}^{b} \underset{\theta}{\stackrel{\epsilon}{\rightleftharpoons}} \Phi_{1}^{b}
$$

The above shows a cascade reaction with non-dimensionalized variables. The Ligand $(\Gamma)$ binds to GPVI $(\varphi)$ to form the GPVI-Ligand Complex $(\Phi)$. The GPVI-Ligand Complex is subsequently phosphorylated $\left(\Phi^{p}\right)$, allowing Cytosolic Syk $(\sigma)$ 
to join and form the Syk-Receptor Complex $\left(\Phi_{0}^{b}\right)$. The Syk-Receptor complex exists at steady state both with Tyrosine 525 phosphorylated $\left(\Phi_{1}^{b}\right)$ and without $\left(\Phi_{0}^{b}\right)$.

\section{Analytical Solution for Equation 2 a}

Numerical integration of the ODEs shows that the ligand concentration is in a pseudo-steady state. After the platelets are exposed to collagen, the rate of change of the ligand is basically zero for the rest of the cascade, and the concentration of the ligand is constant during the period of interest.

Therefore, instead of Equation 1a, the ligand variable is held constant at its initial condition, $1.2 \times 10^{12} \frac{\mathrm{moles}}{\mathrm{m}^{3}}$.

$$
\Gamma(\tau)=\Gamma_{0}
$$

Equation 3a.

Equation $2 \mathrm{~b}$ and Equation $2 \mathrm{c}$ are modified to account for this, and allow Equation $2 \mathrm{a}$ to be removed from the system of ODEs: $\Gamma$ is combined with $\hat{\alpha}_{1}$ to create the $\alpha_{1}$ parameter, $\alpha_{1}=\hat{\alpha}_{1} \Gamma_{0}$.

Analytical Solutions for Equations $2 b$ and $2 c$

These Equations describe the formation of the GPVI-Ligand complex:

$$
\underset{\text { Ligand }}{\Gamma+\varphi} \frac{\hat{\alpha}_{1}}{\alpha_{-1}} \underset{\begin{array}{c}
\text { GPVI-Ligand } \\
\text { Complex }
\end{array}}{\Phi}
$$

In subsequent reactions, $\Phi$ is consumed with rate $\beta$. With the ligand concentration constant, Equations $2 \mathbf{b}$ and $2 \mathbf{c}$ form a closed system. This allows them to be solved exactly by first converting them to matrix form, $\mathbf{x}^{\prime}=\mathbf{A x}$ :

$$
\left(\begin{array}{c}
\frac{d \varphi}{d \tau} \\
\frac{d \Phi}{d \tau}
\end{array}\right)=\left(\begin{array}{cc}
-\alpha_{1} & \alpha_{-1} \\
\alpha_{1} & -\left(\alpha_{-1}+\beta\right)
\end{array}\right) \times\left(\begin{array}{c}
\varphi \\
\Phi
\end{array}\right)
$$

Equation 4.

Finding the eigenvalues and eigenvectors of the matrix above allows an exact, analytical solution to be found (Supplement A).

It was found that

$$
\begin{aligned}
& \varphi(\tau)=c_{1} e^{\lambda_{1} \tau}+c_{2} e^{\lambda_{2} \tau}, \\
& \Phi(\tau)=c_{3} e^{\lambda_{1} \tau}-c_{3} e^{\lambda_{2} \tau}
\end{aligned}
$$

Equation 3b.

Equation 3c.

where $c_{1}=0.137, c_{2}=0.863, c_{3}=0.9698, \lambda_{1}=-6.6145$, and $\lambda_{2}=-68.4855$.

\section{Analytical Approximations for Equations $2 d$ and $2 e$}

The ODEs for cytosolic Syk and phosphorylated GPVI, produced by a previous reaction, form a coupled system, resulting in the formation of the Syk-Receptor complex:

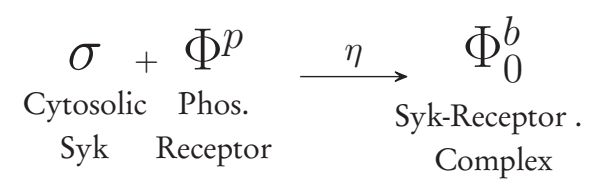




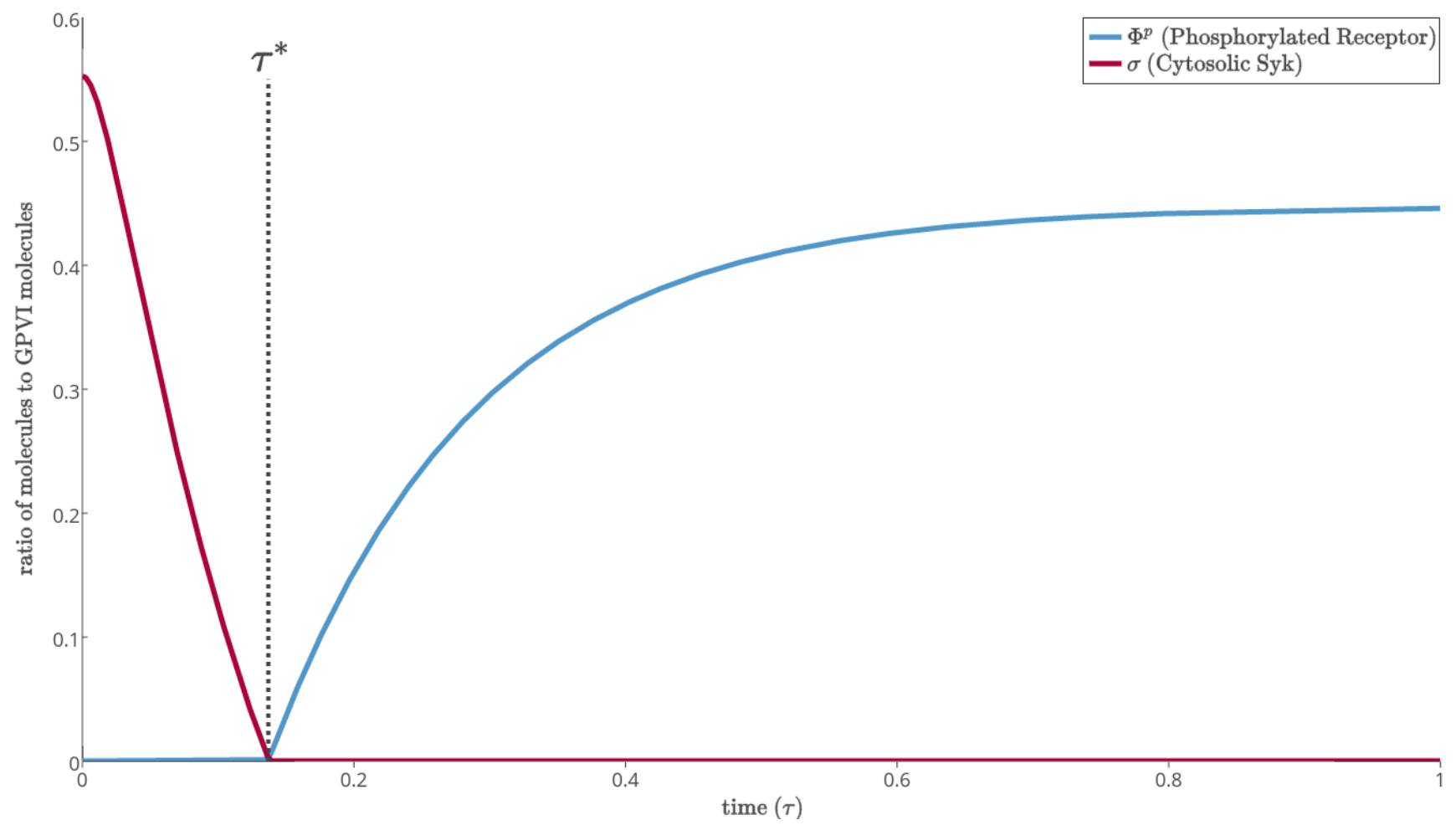

Figure 3. Partitions lending to asymptotic analysis.

The reaction of Syk with phosphorylated GPVI (Equations $\mathbf{2} \mathbf{d}$ and $\mathbf{2 e}$ ) occurs so quickly (with the non-dimensionalized rate constant, $\eta=2.68 \times 10^{5}$ ) that if two reactants are present together, the reaction moves forward. Because of this driving force, phosphorylated GPVI is consumed almost as quickly as it is produced and only begins to accumulate once all of the Syk has reacted. Taking this into account, solutions can be found via asymptotic analysis.

The differential equations can be solved piece-wise with respect to the availability of free $\sigma$. The time at which $\sigma$ is exhausted is labeled $\tau^{*}$ (Figure 3).

Analysis for $\tau<\tau^{*}$

Because $\eta$ is so large, any Syk in solution will consume $\Phi^{p}$ as soon as it is produced. This assumption has implications for both $\frac{d \Phi^{p}}{d \tau}$ (Equation $2 \mathrm{~d}$ ) and $\frac{d \sigma}{d \tau}$ (Equation 2e):

$$
\left(\frac{d \Phi^{p}}{d \tau} \approx 0=\beta \Phi-\eta \Phi^{p} \sigma\right) \Longrightarrow\left(\frac{d \sigma}{d \tau}=-\eta \Phi^{p} \sigma \approx-\beta \Phi\right)
$$

Equation 5.

These assumptions state that changes in $\sigma$ will be proportional to the value of $\Phi(\tau)$, solved previously. Therefore, $\sigma(\tau)$ can be approximated with

$$
\sigma(\tau)=\sigma_{0}-\int_{0}^{\tau} \beta \Phi\left(\tau^{\prime}\right) d \tau^{\prime}, \text { for } 0 \leq \tau<\tau^{*}
$$

Equation 6. 
This gives the following partial approximation for $\sigma(\tau)$ and $\Phi^{p}$ :

$$
\begin{aligned}
\sigma(\tau) & =c_{4} e^{\lambda_{1} \tau}-c_{5} e^{\lambda_{2} \tau}-c_{6}, \\
\Phi^{p} & =0
\end{aligned}
$$

with $\tau \in\left[0, \tau^{*}\right), c_{4}=1.107, c_{5}=0.1069$, and $c_{6}=0.4474$.

Analysis for $\tau \geq \tau^{*}$

As $\tau$ reaches $\tau^{*}, \sigma$ reaches 0 and $\Phi^{p}$ can begin to grow (Figure 3). This allows a similar, but converse, approach to approximating $\Phi^{p}$ and $\sigma$ :

$$
\left(\sigma(\tau) \approx 0, \frac{d \sigma}{d \tau} \approx 0\right) \Longrightarrow \frac{d \Phi^{p}}{d \tau}=\beta \Phi
$$

An approximation to Equation 2d can now be found via integration:

$$
\Phi^{p}(\tau)=\int_{\tau^{*}}^{\tau} \beta \Phi\left(\tau^{\prime}\right) d \tau^{\prime}, \text { for } \tau^{*} \leq \tau \leq 1
$$

Equation 8.

With an approximation of $\Phi^{p}$, piecewise solutions for $\sigma$ and $\Phi^{p}$ can be constructed, with $\tau \in[0,1], c_{4}=1.107, c_{5}=0.1069$, and $c_{6}=0.4474$ :

$$
\begin{aligned}
\Phi^{p}(\tau) & = \begin{cases}0 & \text { for } \tau<\tau^{*} \\
-c_{4} e^{\lambda_{1} \tau}+c_{5} e^{\lambda_{2} \tau}+c_{6} & \text { for } \tau \geq \tau^{*}\end{cases} \\
\sigma(\tau) & = \begin{cases}c_{4} e^{\lambda_{1} \tau}-c_{5} e^{\lambda_{2} \tau}-c_{6} & \text { for } \tau<\tau^{*} \\
0 & \text { for } \tau \geq \tau^{*},\end{cases}
\end{aligned}
$$

Equation 3d.

Equation 3e.

The large size of $\eta$ is reflected in the high affinity that cytosolic Syk has for the GPVI-ligand complex. Also, since cytosolic Syk binds quickly and irreversibly to the GPVI complex, it acts as a molecular clock that determines $\tau^{*}$, which is the time when cytosolic Syk is fully consumed and the concentration of phosphorylated GPVI can grow.

From these equations, the intersection was used to determine $\tau^{*}$, determined from:

$$
\left(\lim _{\tau \rightarrow \tau^{*-}} \sigma(\tau)=\right) 0 \Longrightarrow\left(\tau^{*}=0.137\right)
$$

Equation 9.

\section{Analytical Approximation for Equations $2 f$ and $2 g$}

These equations define the concentrations of $\Phi_{0}^{b}$ and $\Phi_{1}^{b}$, and involve the reaction rates $\epsilon$ and $\theta$ :

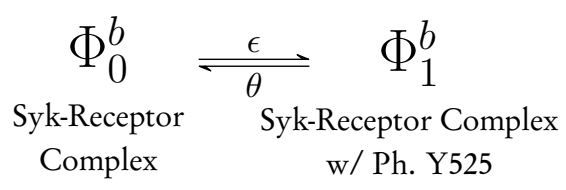

The Syk-Receptor complex is also created at rate $\eta$ as a result of the coupling of cytosolic Syk, $\sigma$ and the phosphorylated receptor, $\Phi^{p}$. We will now solve for $\Phi_{1}^{b}(\tau)$ by eliminating $\Phi_{0}^{b}$ from Equations $2 \mathbf{f}$ and $\mathbf{2 g}$. 
Analysis for $\tau<\tau^{*}$

The previous section established that, for $\tau<\tau^{*}$, we have $\eta \Phi^{p} \sigma \approx \beta \Phi$. This is used in the first step of finding an analytical approximation for $\Phi_{1}^{b}$, solving $\frac{d \Phi_{0}^{b}}{d \tau}$ (Equation $2 \mathbf{f}$ ) for $\Phi_{0}^{b}$ :

$$
\begin{aligned}
\frac{d \Phi_{0}^{b}}{d \tau} & =\beta \Phi-\epsilon \Phi_{0}^{b}+\theta \Phi_{1}^{b} \\
\Phi_{0}^{b} & =\frac{1}{\epsilon}\left(\frac{d \Phi_{1}^{b}}{d \tau}+\theta \Phi_{1}^{b}\right) .
\end{aligned}
$$

Equation 2g*.

After substituting for $\Phi$ from Equation 3c, a solution can be found (see Supplemental C for details):

$$
\Phi_{1}^{b}(\tau)=c_{7}+c_{8} e^{\lambda_{1} \tau}+c_{9} e^{\lambda_{2} \tau}
$$

Equation 10.

with the following constants: $c_{7}=0.126, c_{8}=-0.141, c_{9}=0.0146$.

This solution can be substituted into Equation $2 \mathrm{~g}^{*}$ above, for $\tau<\tau^{*}$. Therefore one has

$$
\Phi_{0}^{b}(\tau)=c_{10} e^{\lambda_{1} \tau}+c_{11} e^{\lambda_{2} \tau}+c_{12}
$$

Equation 11.

with the following $\tau \in\left[0, \tau^{*}\right), c_{10}=-0.9628$, and $c_{11}=0.093, c_{12}=0.867$.

Analysis for $\tau \geq \tau^{*}$

The steady state values for $\Phi_{0}^{b}$ and $\Phi_{1}^{b}$ are well-approximated by their values at $\tau^{*}$ (Supplemental C):

$$
\begin{aligned}
& \Phi_{1}^{b}\left(\tau^{*}\right)=0.069, \\
& \Phi_{0}^{b}\left(\tau^{*}\right)=0.478 .
\end{aligned}
$$

Equation 12a.

Equation 12b.

With these components, solutions can be constructed over the whole domain.

$$
\begin{aligned}
\Phi_{0}^{b}(\tau) & = \begin{cases}c_{10} e^{\lambda_{1} \tau}+c_{11} e^{\lambda_{2} \tau}+c_{12} & \text { if } \tau<\tau^{*} \\
0.478 & \text { if } \tau \geq \tau^{*}\end{cases} \\
\Phi_{1}^{b}(\tau) & = \begin{cases}c_{7}+c_{8} e^{\lambda_{1} \tau}+c_{9} e^{\lambda_{2} \tau} & \text { if } \tau<\tau^{*} \\
0.069 & \text { if } \tau \geq \tau^{*}\end{cases}
\end{aligned}
$$

Equation 3f.

Equation 3g. 


\section{Full Analytical Solutions for Platelet Activation}

For the series of reactions (illustrated in Figure 1) the explicit solutions are

$$
\begin{aligned}
\Gamma(\tau) & =\Gamma_{0}, \\
\varphi(\tau) & =c_{1} e^{\lambda_{1} \tau}+c_{2} e^{\lambda_{2} \tau}, \\
\Phi(\tau) & =c_{3} e^{\lambda_{1} \tau}-c_{3} e^{\lambda_{2} \tau}, \\
\Phi^{p}(\tau) & =\left\{\begin{array}{ll}
0 & \text { for } \tau<\tau^{*} \\
-c_{4} e^{\lambda_{1} \tau}+c_{5} e^{\lambda_{2} \tau}+c_{6} & \text { for } \tau \geq \tau^{*}
\end{array},\right. \\
\sigma(\tau) & =\left\{\begin{array}{ll}
c_{4} e^{\lambda_{1} \tau}-c_{5} e^{\lambda_{2} \tau}-c_{6} & \text { for } \tau<\tau^{*} \\
0 & \text { for } \tau \geq \tau^{*}
\end{array},\right. \\
\Phi_{0}^{b}(\tau) & =\left\{\begin{array}{ll}
c_{10} e^{\lambda_{1} \tau}+c_{11} e^{\lambda_{2} \tau}+c_{12} & \text { for } \tau<\tau^{*} \\
0.478 & \text { for } \tau \geq \tau^{*}
\end{array},\right. \\
\Phi_{1}^{b}(\tau) & = \begin{cases}c_{7}+c_{8} e^{\lambda_{1} \tau}+c_{9} e^{\lambda_{2} \tau} & \text { for } \tau<\tau^{*} \\
0.069 & \text { for } \tau \geq \tau^{*},\end{cases}
\end{aligned}
$$

Equation 14a.

Equation 14b.

Equation 14c.

Equation 14d.

Equation 14e.

Equation 14f.

Equation 14g.

with $\lambda_{1}=-6.6145$, and $\lambda_{2}=-68.4855, c_{1}=0.137, c_{2}=0.863, c_{3}=0.9698, c_{4}=1.107, c_{5}=0.1069, c_{6}=0.4474$, $c_{7}=0.126, c_{8}=-0.141, c_{9}=0.0146, c_{10}=-0.9628, c_{11}=0.093$, and $c_{12}=0.867$, and $\tau^{*}=0.137$.

From Figure 4, the analytic solutions compare favorably to the numerical ones.

\section{CONCLUSIONS}

The system of ODEs from Dunster et al.'s Model A is simplified by first finding its dimensionless equivalent. This process allowed different concentrations and rate kinetics to be directly compared. In addition, the approximate analytical solutions for all the ODEs were found. These solutions are more accessible than the coupled system of differential equations, and each component can now be viewed separately.

Equation 14a shows that the ligand concentration is held constant at its initial value. This is permissible since the right hand side of Equation 2a, the ligand ODE, reaches zero shortly after time zero. It also shows the extent to which phosphorylated Syk functions as a biological clock. When $t$ is equal to approximately 34 seconds, cytosolic Syk is depleted and allows the signal cascade to reach a pseudo steady-state.

The asymptotic, approximate analytic solutions favorably compare to Dunster et al.'s numerical solutions. They maintain its accuracy and scope while providing solutions that are explicit and easier to understand. Further, the underlying biology is more readily exposed; $\tau^{*}$, the effective phosphorylation time scale, marks a clear threshold in the cascade.

The timing mechanism, especially, is shown by the distinct changes in concentrations seen in Figure 4. At $\tau^{*}$, the effective phosphorylation time scale, there is a switch in qualitative behavior for many of the reactants. This is the time that it takes for several of the reactants, bound, phosphorylated, and cytosolic Syk, to reach steady state. These steady state levels then feed into subsequent downstream reactions that are not described in this simplified model. The explicit solutions indicate a coordinated timing mechanism that either activates or deactivates complexes within this cascade.

The experimental data, to which Dunster et al.'s models were parameterized, indicates an overshoot and subsequent undershoot of phosphorylated Syk at $\tau^{*}$. This often arises in systems featuring strong feedback mechanisms. ${ }^{17}$ In fact, Dunster et al. offers models of increasing complexity that more accurately capture this phenomenon. ${ }^{1}$ This is accomplished by introducing more granularity to the phosphorylation of cytosolic Syk on Y525, and an additional phosphorylation site on Y323. 


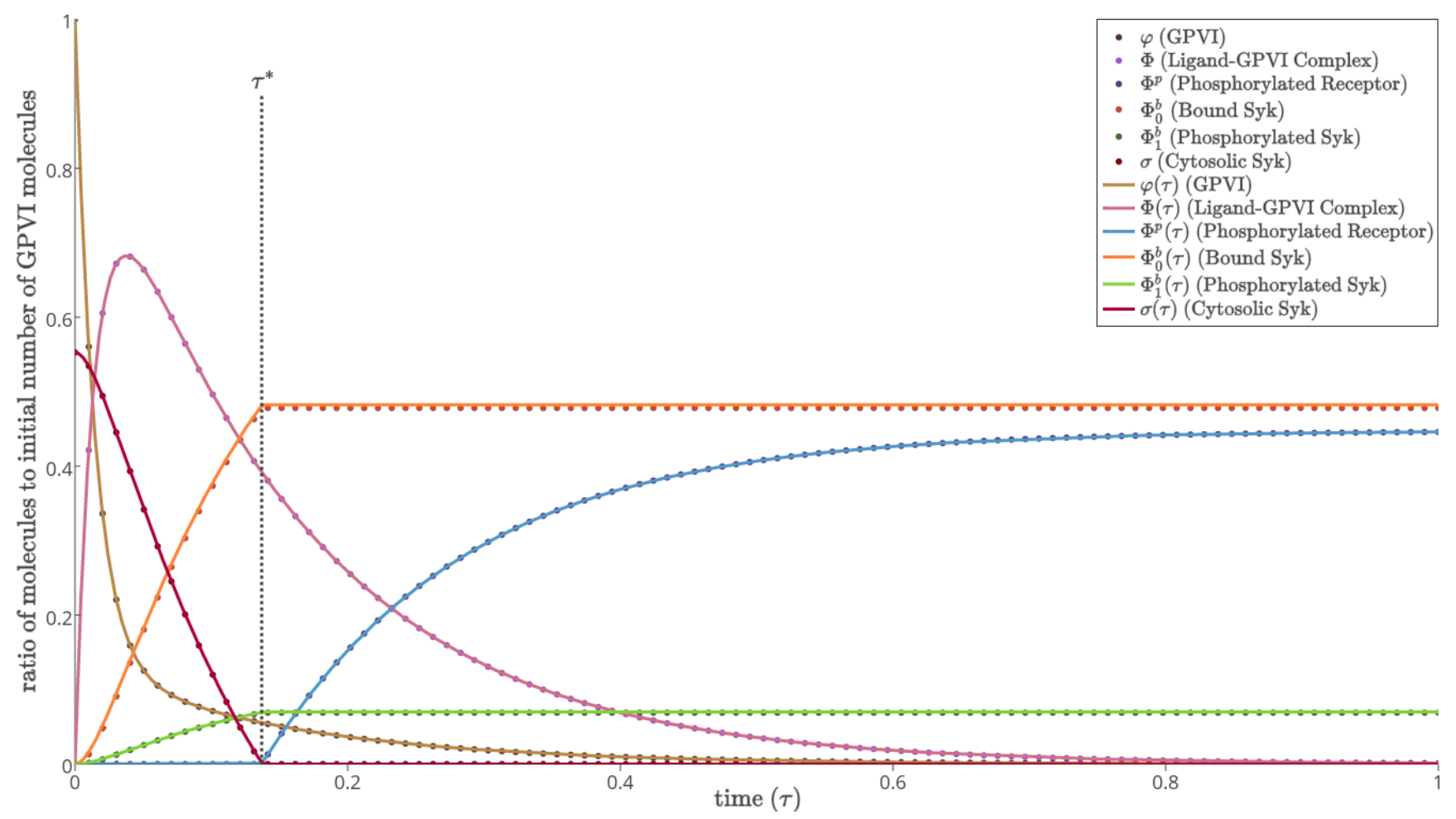

Figure 4. Approximate analytical solutions for the ODEs, $\varphi(\tau), \Phi(\tau), \sigma(\tau), \Phi^{p}(\tau), \Phi_{1}^{b}(\tau)$, and $\Phi_{0}^{b}(\tau)$, with respect to time. The dimensionless versions of Dunster et al.'s ODEs for the GPVI $(\varphi)$, the GPVI complex $(\Phi)$, phosphorylated GPVI ( $\left.\Phi^{p}\right)$, and the cytosolic Syk $(\sigma)$ were solved to create functions dependent on time. The dotted lines represent the numerical solutions from Dunster et al. while the solid lines are the analytical solutions. Cytosolic Syk serves as a molecular clock, marking the timescale of effective phosphorylation. Its depletion at $\tau^{*}$ correlates to a dramatic change in the cascade's dynamics.

However, these models do not include additional steps in the platelet's activation cascade. An interesting extension to this work would be to use our analytic approximations for the ligand, GPVI, and Syk (Equations 14a-14e) as inputs to more complicated models of platelet activation, such as Models B and C of Dunster et al. ${ }^{1}$ 


\section{REFERENCES}

1. Dunster, Joanne L., Mazet, Francoise, Fry, Michael J., Gibbins, Jonathan M., and Tindall, Marcus J. Regulation of Early Steps of GPVI Signal Transduction by Phosphatases: A Systems Biology Approach. PLOS Computational Biology, 11(11):1-26, 2015.

2. Watson, S. P., Auger, J. M., McCarty, O. J. T. and Pearce, A. C. GPVI and integrin AlphaIIbBeta3 signaling in platelets. Journal of Thrombosis and Haemostasis, 3(8): 1752-1762, 2005.

3. Ruggeri, ZM. Platelets in atherothrombosis.Nature Medicine, 8(11): 1227-1234, 2002.

4. Stepanek, O, Draber, P, Drobek, A, Horejsi, V and Brdicka, T. Nonredundant Roles of Src-Family Kinases and Syk in the Initiation of B-Cell Antigen Receptor Signaling. Journal of Immunology,190(4): 1807-1818, 2013.

5. Purvis, Jeremy E., Chatterjee, Manash S., Brass, Lawrence F. and Diamond, Scott L. A molecular signaling model of platelet phosphoinositide and calcium regulation during homeostasis and P2Y1 activation. Blood, 112(10):4069-4079, 2008.

6. Thomas, Dafydd H., Getz, Todd M., Newman, Tiffanny N., Dangelmaier, Carol A., Carpino, Nick, Kunapuli, Satya P., Tsygankov, Alexander Y., and Daniel, James L. A novel histidine tyrosine phosphatase, TULA-2, associates with Syk and negatively regulates GPVI signaling in platelets. Blood, 116(14): 2570-2578, 2010.

7. Dangelmaier, Carol A., Quinter, Patericia G., Jin, Jianguo, Tsygankov, Alexander Y., Kunapuli, Satya P., and Daniel, James L. Rapid ubiquitination of syk forllowing GPVI activation in platelets. Blood, 105(10): 3918-3924, 2005.

8. Senis, Yotis A., Mazharian, Alexandra, and Mori, Jun. Src family kinases: at the forefront of platelet activation. Blood, 124(13) 2013-2024, 2014.

9. Tonks, Nicholas K. Protein tyrosine phosphatases - from housekeeping enzymes to master regulators of signal transduction. FEBS Journal 280(2): 346-378, 2013.

10. Tsang, Emily, Giannetti, Anthony M., Shaw, David, Dinh, Marie, Tse, Joyce K. Y., Gandhi, Shaan, Ho, Hoangdung, Wang, Sandra, Papp, Eva and Bradshaw, J. Michael. Molecular Mechanism of the Syk Activation Switch. Journal of Biological Chemistry, 283(47):32650-32659, 2008.

11. Heemskerk, JWM, Bevers, EM, and Lindhout, T. Platelet activation and blood coagulation. Thrombosis and Haemostasis, 88(2): 186-193, 2002.

12. Hognestad, Aina, Michelsen, Annika, Brosstad, Frank, Damås, Jan K, Holm, Torbjørn, Simonsen, Svein, Kjekshus, John K, Aukrust, Pål, and Andreassen, Arne K. Platelet activation in heart transplant recipients. Clinical Transplantation, 18(2): 142-147, 2004.

13. Robinson, SD, Harding, SA, Cummins, P, Din, JN, Sarma, J, Davidson, I, Fox, KAA, Boon, NA, and Newby, DE. Functional interplay between platelet activation and endothelial dysfunction in patients with coronary heart disease. Platelets 17(3): 158-162, 2006.

14. Kreis, T. and Vale, R. Guidebook to the Extracellular Matrix, Anchor, and Adhesion Proteins. A Sambrook \& Tooze publication at Oxford University Press.Oxford University Press, 1999.

15. Nieswandt, B., Pleines, I., and Bender, M. Platelet adhesion and activation mechanisms in arterial thrombosis and ischaemic stroke. Journal of Thrombosis and Haemostasis, 9:92-104, 2011.

16. Miura, Y., Takahashi, T., Jung, S., Moroi, M. Analysis of the Interaction of Platelet Collagen Receptor Glycoprotein VI (GPVI) with Collagen. Journal of Biological Chemistry, 277:46197-46204, 2002.

17. Alon, U. Network motifs: Theory and experimental approaches. Nature Reviewes.Genetics. 8(6):450-61, 2007. doi: http://dx.doi.org.udel.idm.oclc.org/10.1038/nrg2102. 


\section{SUPPLEMENT A: ANALYTICAL SOLUTIONS FOR $\phi$ AND $\Phi$}

The equations for $\phi$ and $\Phi$ can be solved as an independent system, separate from the other ODEs.

$$
\begin{aligned}
& \frac{d \varphi}{d \tau}=-\alpha_{1} \varphi+\alpha_{-1} \Phi \\
& \frac{d \Phi}{d \tau}=\alpha_{1} \varphi-\alpha_{-1} \Phi-\beta \Phi
\end{aligned}
$$

Equation 15a.

Equation 15b.

In matrix form, $\mathbf{x}^{\prime}=\mathbf{A x}$ :

$$
\left(\begin{array}{c}
\frac{d \varphi}{d \tau} \\
\frac{d \Phi}{d \tau}
\end{array}\right)=\left(\begin{array}{cc}
-\alpha_{1} & \alpha_{-1} \\
\alpha_{1} & -\left(\alpha_{-1}+\beta\right)
\end{array}\right) \times\left(\begin{array}{c}
\varphi \\
\Phi
\end{array}\right) .
$$

Equation 16.

Solving for the eigenvalues of A yields the approximate solutions: $\lambda_{1}=-6.6145, \lambda_{2}=-68.4855$, with corresponding eigenvectors:

$$
\mathbf{v}_{\mathbf{1}}=\left(\begin{array}{c}
0.14003 \\
0.99015
\end{array}\right), \mathbf{v}_{\mathbf{2}}=\left(\begin{array}{c}
-0.66473 \\
0.74709
\end{array}\right)
$$

Equation 17.

Therefore, solutions have the form:

$$
\left(\begin{array}{l}
\varphi \\
\Phi
\end{array}\right)=d_{1} \mathbf{v}_{\mathbf{1}} e^{\lambda_{1} \tau}+d_{2} \mathbf{v}_{\mathbf{2}} e^{\lambda_{2} \tau}
$$

Equation 18.

$d_{1}$ and $d_{2}$ can be found by solving at the initial conditions $\varphi(0)=1$ and $\Phi(0)=0$.

$$
\begin{aligned}
1 & \approx 0.14003 d_{1}-0.66473 d_{2} \\
0 & \approx 0.99015 d_{1}+0.74709 d_{2} \\
d_{1} & \approx 0.97941 \\
d_{2} & \approx-1.29806
\end{aligned}
$$

Therefore, the solutions for $\varphi$ and $\Phi$ are.

$$
\begin{aligned}
& \varphi \approx 0.13715 e^{-6.6145 \tau}+0.86285 e^{-68.4854 \tau} \\
& \Phi \approx 0.96976 e^{-6.6145 \tau}-0.96976 e^{-68.4854 \tau}
\end{aligned}
$$

\section{SUPPLEMENT B: ANALYTICAL SOLUTIONS FOR $\Phi^{P}$ AND $\sigma$}

Approximate analytical solutions can be found for $\Phi^{p}$ and $\sigma$ via asymptotic analysis.

Because $\eta$ is so large, any Syk in solution will consume $\Phi^{p}$ as soon as it is produced. $\tau^{*}$ denotes the time when $\sigma$ reaches zero and $\Phi^{p}$ can begin to grow. This allows $\Phi^{p}$ and $\sigma$ to be defined piece-wise with respect to $\tau^{*}$ and allow approximate solutions to be obtained.

$$
\begin{gathered}
\sigma(\tau)= \begin{cases}\sigma_{0}-\int_{0}^{\tau} \beta \Phi\left(\tau^{\prime}\right) d \tau^{\prime} & \text { if } 0 \leq \tau<\tau^{*} \\
0 & \text { if } \tau^{*} \leq \tau \leq 1\end{cases} \\
\Phi^{p}(\tau)= \begin{cases}0 & \text { if } 0 \leq \tau<\tau^{*} \\
\int_{\tau^{*}}^{\tau} \beta \Phi\left(\tau^{\prime}\right) d \tau^{\prime} & \text { if } \tau^{*} \leq \tau \leq 1\end{cases}
\end{gathered}
$$

Equation 19.

Equation 20.

The value of $\tau^{*}$ can be found by solving $\sigma\left(\tau^{*}\right)=0$.

$$
\begin{aligned}
0 & =\sigma_{0}-\int_{0}^{\tau^{*}} \beta \Phi\left(\tau^{\prime}\right) d \tau^{\prime} \\
& =0.5526-\int_{0}^{\tau^{*}} 7.55\left(0.9698 e^{-6.6145 \tau^{\prime}}-0.9698 e^{-68.4855 \tau}\right) d \tau^{\prime} \\
\Longrightarrow-0.4474 & =-1.107 e^{-6.6145 \tau^{*}}+0.1069 e^{-68.4855 \tau^{*}} \\
\Longrightarrow \tau^{*} & =0.137
\end{aligned}
$$


a particular solution to $\Phi_{1}^{b}$ can be assumed to take the form:

$$
\Phi_{1}^{b}(\tau)=A e^{-6.6145 \tau}+B e^{-68.4855 \tau} .
$$

Equation 22.

Substituting into Equation A2 and comparing coefficients resulted in the following solutions of $A$ and $B$ :

$$
\begin{aligned}
& A=-0.141 \\
& B=0.0146
\end{aligned}
$$

Equation 23.

Equation 24.

Now, the solution to the differential equation is a combination of the homogeneous solution and the particular solution.

$$
\Phi_{1}^{b}=f_{1}+f_{2} e^{-1010.75 \tau}-0.141 e^{-6.6145 \tau}+0.0146 e^{-68.4855 \tau}
$$

Equation 25.

Solutions for the constants are found by taking the derivative and using the initial values of $\Phi_{1}^{b}(\tau)$ and $\frac{d \Phi_{1}^{b}}{d \tau}$.

$$
\begin{aligned}
& f_{1}=0.126 \\
& f_{2}=6.629 \times 10^{-5}
\end{aligned}
$$

Equation 26.

Equation 27.

The final solution is:

$$
\Phi_{1}^{b}(\tau)=0.126-6.629 \times 10^{-5} e^{-1010.25 \tau}-0.141 e^{-6.6145 \tau}+0.0146 e^{-68.4855 \tau}
$$

Equation 28.

The above solution is only accurate when $0 \leq \tau<\tau^{*}$. To determine the steady state value, the equation must be evaluated at $\tau=\tau^{*}$, where $\tau^{*}=0.137$.

$$
\begin{aligned}
\Phi_{1}^{b}\left(\tau^{*}\right) & =0.126-6.629 \times 10^{-} 5 e^{-1010.25 \tau^{*}}-0.141 e^{-6.6145 \tau^{*}}+0.0146 e^{-68.4855 \tau^{*}} \\
& =0.069
\end{aligned}
$$

Equation 29.

Equation 30.

Equation 31.

The final equation for $\Phi_{1}^{b}(\tau)$ is as follows.

$$
\Phi_{1}^{b}(\tau)= \begin{cases}0.126-6.629 \times 10^{-5} e^{-1010.75 \tau}-0.141 e^{-6.6145 \tau}+0.0146 e^{-68.4855 \tau} & \text { if } 0 \leq \tau<\tau^{*} \\ 0.069 & \text { if } \tau^{*} \leq \tau \leq 1\end{cases}
$$

Equation 32.

Next, an analytical approximation for $\Phi_{0}^{b}$ is determined from the original equation, Equation $2 \mathrm{~g}$. Since $\Phi_{1}^{b}$, is now known, $\Phi_{0}^{b}$ can be solved by substituting the solution for $\Phi_{1}^{b}$ into Equation $2 \mathrm{~g}^{*}$.

$$
\Phi_{0}^{b}=\frac{1}{\epsilon}\left(\frac{d}{d \tau} \Phi_{1}^{b}+\theta \Phi_{1}^{b}\right)
$$

Equation $2 \mathrm{~g} *$

Solving for $\Phi_{0}^{b}$ when $\tau^{*}=0.137$

$$
\begin{aligned}
\Phi_{0}^{b}(0.137) & =-0.9628 e^{-0.9062}+0.093 e^{-9.383}+6.629 \times 10^{-5} e^{-138.49}+0.00272 e^{-17.57}+0.867 \\
\Phi_{0}^{b}\left(\tau^{*}\right) & =0.478
\end{aligned}
$$

Equation 33.

Equation 34.

The final equation comes out to be:

$$
\Phi_{0}^{b}(\tau)= \begin{cases}-0.9628 e^{-6.6145 \tau}+0.093 e^{-68.4855 \tau}+6.629 \times 10^{-5} e^{-1010.75 \tau}+0.00272 e^{-128.25 \tau}+0.867 & \\ 0.478 & \text { if } 0 \leq \tau<\tau^{*} \\ & \text { if } \tau^{*} \leq \tau \leq 1\end{cases}
$$

Equation 35. 
Note: Both $\Phi_{0}^{b}$ and $\Phi_{1}^{b}$ include an $e^{-1010.75 \tau}$ term that does not contribute significantly to the solution. Removing this term from both equations simplifies them to:

$$
\begin{aligned}
\Phi_{1}^{b}(\tau) & =\left\{\begin{array}{lll}
0.126-0.141 e^{-6.6145 \tau}+0.0146 e^{-68.4855 \tau} & \text { if } \tau \leq \tau^{*} \\
0.069 & \text { if } \tau>\tau^{*}
\end{array}\right. \\
\Phi_{0}^{b}(\tau) & =\left\{\begin{array}{lll}
-0.9628 e^{-6.6145 \tau}+0.093 e^{-68.4855 \tau}+0.00272 e^{-128.25 \tau}+0.867 & \text { if } 0 \leq \tau<\tau^{*} \\
0.478 & \text { if } \tau^{*} \leq \tau \leq 1 & \text { Equation 36. }
\end{array}\right.
\end{aligned}
$$

\section{ABOUT THE STUDENT AUTHORS}

Rachel Austin recently graduated from the University of Delaware with a Bachelor of Science in Quantitative Biology and a minor in Dance and is currently serving as an AmeriCorps member with City Year Philadelphia.

Scott Fones is currently a senior at the University of Delaware, pursuing a Bachelor of Science in Quantitative Biology and minoring in Computer Science.

Dominic Santoleri graduated from the University of Delaware in 2017 with Bachelors of Science in Biochemistry and in Quantitative Biology. He is currently pursuing a $\mathrm{PhD}$ in Biochemistry and Molecular Biophysics at the University of Pennsylvania.

Kaitlyn Thomesen recently graduated from the University of Delaware with a Bachelor of Science in Quantitative Biology and Economics and a minor in Chemistry and Biochemistry. She is currently pursuing her Master of Business Administration with a concentration in Healthcare Management at the University of Delaware.

\section{PRESS SUMMARY}

The ordinary differential equations proposed in Model A of Regulation of Early Steps of GPVI Signal Transduction by Phosphatase: A Systems Biology Approach by JL Dunster were simplified through the process of non-dimensionalization. The approximated analytical solutions were found and compared against the numerical solutions. With this simplification, a cross comparison for activation kinetics in this phenomenon was performed, allowing for a better understanding of the signal regulation pathway specifically, thereby exposing an effective phosphorylation time scale of about 34 seconds. 
Print Edition ISSN 1536-4585

Online Edition ISSN 2375-8732 